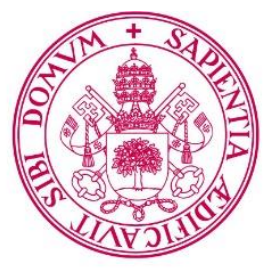

Universidad deValladolid

DOCTORAL THESIS

\title{
BIOLOGICALLY RELEVANT SUBGROUPS WITHIN THE SCHIZOPHRENIA SYNDROME
}

This dissertation is submitted by Alba Lubeiro Juárez

for the degree of Doctor of Philosophy at the

University of Valladolid

Supervisor Dr. Vicente Molina Rodríguez

Valladolid, Spain, 2018 



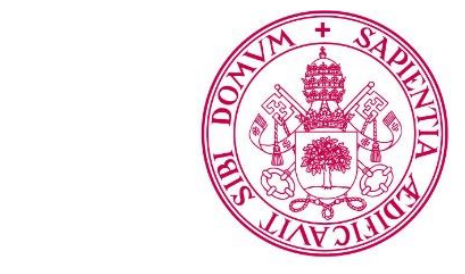

Universidad deValladolid

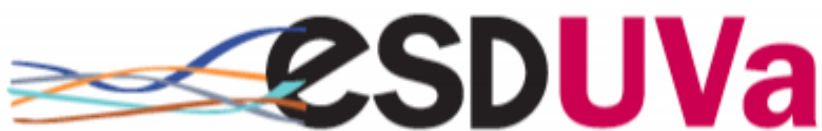

Escuela de Doctorado Universidad de Valladolid

PROGRAMA DE DOCTORADO EN INVESTIGACIÓN BIOMÉDICA

TESIS DOCTORAL

\section{BIOLOGICALLY RELEVANT SUBGROUPS WITHIN THE SCHIZOPHRENIA SYNDROME}

Presentada por Alba Lubeiro Juárez para optar al grado de Doctora por la Universidad de Valladolid

Dirigida por Dr. Vicente Molina Rodríguez

Valladolid, España, Octubre de 2018 

Título: Biologically relevant subgroups within the schizophrenia syndrome

Autor: Alba Lubeiro Juárez

Dirigida por: Vicente Molina Rodríguez

Tutorizada por: Diego Sánchez

Departamento: Departamento Pediatría e inmunología, Obstetricia y

Ginecología, Nutrición y Bromatología, Psiquiatría e Historia de la Ciencia

Esta tesis fue leída en la facultad de Medicina en la Universidad de Valladolid el día , estando compuesto el tribunal calificador por :

Presidente:

Secretario:

Vocal :

Obteniendo la calificación de:

En Valladolid, España, a de de 201 

A mi madre. 

A veces la enfermedad puede enseñarnos lo que tiene la vida de valioso.

- Oliver Sacks -

Todo lo que ocurre en el cerebro es biología y todo lo que ocurre en la mente, ocurre a través del cerebro.

- Joseph le Doux - 

Suelta lo malo y déjalo correr, que estás en vuelo.

Óyeme, ponte en talla y vámonos de aquí para el cielo.

Vámonos a construir lo nuevo, a ponerlo todo boca abajo, a comer el mundo con los dedos, a romper lo negro en mil pedazos. Con amor que es todo lo que tengo y estas ganas locas de entregarlo. Para cambiar esta vida de perros, para vivir el tiempo más humano.

Vueltas

- Amparo Sánchez y Pascual Cantero - 



\section{Agradecimientos / Greetings}

A mi maestro, Vicente Molina, que me dio su confianza sin conocerme, por creer en mí y apoyarme. Por darme esta gran oportunidad y enseñarme los entresijos de la investigación. Ha sido un honor para mí poder trabajar contigo, de verdad, gracias.

A las fuentes de financiación que han permitido la realización de esta Tesis Doctoral: una beca predoctoral de la Junta de Castilla y León y el Fondo Social Europeo, una beca de estancia nacional de la Universidad de Valladolid y una beca de estancia internacional de European Molecular Biology Organization (EMBO Short-term fellowship).

A Diego Sánchez, Lola Ganfornina y el resto del Laboratorio Lazarillo. Por dejarnos trabajar en su laboratorio, sin condiciones. Pero sobre todo por el cariño con el que siempre me han tratado, por sus consejos y apoyo. Por su gran amor a la enseñanza, seguiré vuestro ejemplo.

A mi coordinadora de doctorado en Investigación Biomédica, Teresa Pérez, por preocuparse, animarme y estar ahí para cualquier duda.

A todo el personal de FIDMAG y la Universidad de Barcelona, por acogerme con los brazos abiertos. En especial a Mar Fatjó-Vilas, mi segunda maestra, María Guardiola y Carmen Almodóvar. ¡Qué tres meses más maravillosos pasé con vosotras! A veces el ideal de que el trabajo puede ser divertido y alegre, además de productivo, se cumple.

To Wroclaw Medical University, Department of Genetics, specifically to Błażej Misiak, Elżbieta Szmida, Małgorzata Krzystek-Korpacka, Bartlomiej Stanczykiewicz and Filip Stramecki. Thank you for teaching me, helping me and making me feel at home in your country.

A todo el equipo de Resonancia Magnética de la UVa y el Sacyl, en especial a Margarita Rodríguez, por colaborar en todo lo posible y hacer las cosas tan fáciles y por supuesto, por ser siempre encantadora conmigo. 
A todo el personal del Hospital de Día de Psiquiatría. Sobre todo a Rosa, por hablarme de Vicente, sin ella no estaría aquí; a Begoña, por su colaboración y amabilidad, da gusto trabajar con personas así; y a Eva Sotelo, también por su colaboración y eficacia.

A los centros que han colaborado en nuestros proyectos: Hospital Universitario de Álava (Vitoria), Hospital de Cruces (Bilbao) y Hospital 12 de Octubre (Madrid). Gracias principalmente a Aitor Palomino, Rafael Segarra, Mariana Bustillo y Javier Sanz.

A Cristina Rueda, por su colaboración e implicación. Gracias a ella pudimos sacar adelante el artículo más importante de esta tesis.

A los Residentes y resto de Médicos que han colaborado, de una u otra manera, en este proyecto: Marta Ayuso, Henar de la Red, Adrián Alonso, Nieves de Uribe, Rebeca Hernández, Marta Hernández, Marta García, Cristina Domínguez, Óscar Martín, Alicia Rodríguez, Laura Gallardo, Pilar del Valle, Patricia Marques, José Antonio Blanco y Fernando Uribe. Quiero hacer una mención especial a Benjamín Cea y Aldara Álvarez, con quien he compartido momentos de trabajo pero también inquietudes, preocupaciones, risas y alegrías. ¡Ojalá podamos mantener nuestra amistad durante mucho tiempo!

A todos los Ingenieros que han participado en los artículos incluidos en esta tesis, Rodrigo de Luis, Javier Gómez, Alejandro Bachiller, Jesús Poza, Roberto Hornero y Pablo Núñez, sin vosotros nada de esto sería posible, me siento afortunada de haber podido trabajar juntos. Alejandro, fuiste un gran apoyo en los inicios de este camino, gracias por ser tan majo y tener siempre una sonrisa. Javier, gracias por explicarme conceptos "ingenieriles" con tanta paciencia, por ayudarme en lo posible y estar disponible en cualquier momento.

A los estudiantes de Medicina cuyos trabajos fin de grado han estado entrelazados con parte del trabajo de esta tesis: Óscar Soto, Jorge Blanco, Daniel Ortega, Miguel Jiménez e Isabel Blanco.

A Vanessa Suazo, por sus consejos y ayuda en la primera etapa de mi formación.

A mis actuales compañeros, Sabela y Álvaro, que me han acompañado y apoyado en esta última parte de la tesis y hacen que me sienta parte de un equipo de verdad. 
A la banda sonora que me ha acompañado durante esta tesis: Amparo Sánchez, Celtas Cortos, Eskorzo, Depedro, La M.O.D.A, Kase O, Natalia Lafourcade, Lila Downs, Leonard Cohen, Bob Dylan, Gregory Porter y muchos más.

To my friends Tita and Kuba. I feel fortunate to have met you. I will never forget the three great months we were together in Wroclaw. We must keep in touch despite the distance between our homes.

A mis amigos Pucelanos, en primer lugar a Clara, compañera de camino desde hace muchos años, sin tu ejemplo y ayuda quizá no habría llegado aquí. Ahora las dos lo hemos conseguido, isomos doctoras!. En segundo lugar a Nati, María, David y Sara. Gracias por estar, por las cenas, los bailes, las risas y, en general, los buenos momentos. Qué suerte tengo de teneros cerca. Por último, a Christopher, por su ayuda con las correcciones del inglés.

A mis amigos Leoneses "Los Jetas", con los que recorrí las primeras etapas de mi formación universitaria. Porque nuestras pequeñas y grandes escapadas me han dado fuerzas para realizar esta tesis y lo mejor, permiten que sigamos unidos.

A mi padre y abuelos, por darme los recursos y el apoyo para poder estudiar.

A mi familia no de sangre, pero sí de corazón, por orden de llegada a mi vida: Celia, Santi, Laura, Samuel, Inés y Manolo. Algunos formáis parte de estos artículos como "conejillos de indias". Gracias por vuestro apoyo, por estar a mi lado siempre y porque la vida es mejor teniéndoos cerca.

A Julio, porque tú haces que la vida se me vuelva de colores. Esto es un logro compartido, de ambos.

A mi madre, por enseñarme a vivir, a ser valiente, a luchar, a levantarme en cada tropiezo. Por transmitirme el amor de la cultura y la educación. Esta tesis es por y para ti.

A los controles y pacientes que dieron su tiempo para formar parte de este proyecto. $Y$ en general, a todos los pacientes que sufren o han sufrido enfermedades mentales y a sus familiares. Este es mi pequeño granito de arena. 



\section{Abstract}

Diagnostic criteria for schizophrenia are based on signs and symptoms and do not take into account biological abnormalities. Therefore, current diagnoses are subjective and unspecific, promoting enormous differences in clinical presentations among patients with the same diagnosis. On the other hand, lots of biological processes have been studied in relation to schizophrenia. Some of the findings support neurotransmitters alterations, neuroanatomical changes, connectivity disruptions, immune dysregulation, genetic or environmental risk factors. Nevertheless, research findings are inconclusive, very heterogeneous and difficult to replicate. Thus, schizophrenia aetiology is not well understood in spite of the huge efforts made by researches.

Different evidence suggests that schizophrenia is not a homogeneous disease but a syndrome comprising patients with different biological disruptions. In order to cope with this heterogeneity, the low replication rate of research findings and the lack of biological validity of current diagnosis is necessary to incorporate new research approaches that can eventually translate in a change in psychiatric diagnosis.

In the present thesis, we aimed to explore the existence of biological subgroups within schizophrenia patients by using data from structural and functional brain connectivity as well as a genetic information. It includes five articles with sample sizes from 27 to 121 schizophrenia patients and 27 to 144 healthy controls. All patients were diagnosed according to DSM-IV or V criteria and their symptoms were scored using the Positive and Negative Syndrome Scale (PANSS).

Structural connectivity was assessed in two different ways. Firstly, using structural magnetic resonance imaging (MRI) we extracted measures of cortical curvature. Secondly, diffusion magnetic resonance imaging (dMRI) was used to obtain values of streamline count and fractional anisotropy in white matter tracts connecting a priori selected regions. Functional connectivity was calculated using electroencephalography (EEG) recordings during the performance of an auditive odd-ball task, in which 
participants were instructed to respond to infrequent targets while ignoring other stimuli. Then, small-worldness ( $S W n$ ) index, which quantifies the efficiency of the global electrical network, was calculated at two temporal windows: before and after the target stimulus onset (baseline/pre-stimulus and response window, respectively). We focused our study on the SWn difference between pre-stimulus and response windows as a measure of modulation efficiency.

Regarding genetics, single nucleotide polymorphisms (SNP), related to the risk of schizophrenia and involved in important functions of connectivity such as myelination, excitatory/inhibitory balance or signal transduction, were genotyped. In particular, we studied three SNPs in neuregulin 1 gene (NRG1; rs6468119, rs6994992 and rs7005606), one in calcium voltage-gated channel subunit alpha1C gene (CACNA1C; rs1006737) and another in potassium voltage-gated channel subfamily $\mathrm{H}$ member 2 gene $(K C N H 2$, rs3800779). Individuals were classified as risk-allele carriers or non-carriers for each SNP.

In an initial study, we used a data-driven method to explore the potential of neuroanatomical MRI data for patients classification. Larger cortical curvature identified a subgroup of $20 \%$ schizophrenia patients. This subgroup also showed decreased metabolic rates in the thalamus and cingulate, a lack of the expected increase in metabolic rates in the putamen with antipsychotics and no improvement of negative symptoms.

In a subsequent study, we demonstrated that prefrontal cortical curvature was related to prefrontal white matter connections with anterior cingulate and superior frontal cortex. Finally, in the following study, we found structural connectivity disruptions between the prefrontal cortex and the anterior cingulate, thalamus, caudate and hippocampus in schizophrenia patients.

On the other hand, in a first study relating functional connectivity and genetics, NRG1 polymorphisms were associated with impaired SWn modulation in healthy controls. Subsequently, in a second study, CACNA1C polymorphism was not associated with SWn modulation either in patients or in controls and $K H C N 2$ risk allele $(A)$ was related to abnormal SWn modulation in patients. Noteworthy, patients carrying the KCNH2 
rs3800779 risk allele was characterised by an opposite SWn modulation in comparison to patients not carrying the risk allele and healthy controls.

In summary, this thesis analyses the existence of biological distinguishable subgroups among schizophrenia patients. Our result supports the utility of data-driven neuroimaging and genetic-based approaches looking for biological valid subgroups in the study of mental diseases. Our findings do not seem to be an artefact of the classifying method selected and they appear to be closer to clinically relevant groups. We suggest that similar methods will be useful in the future to reframe psychiatric diagnoses and to lead to new and more productive research strategies. 



\section{Contents}

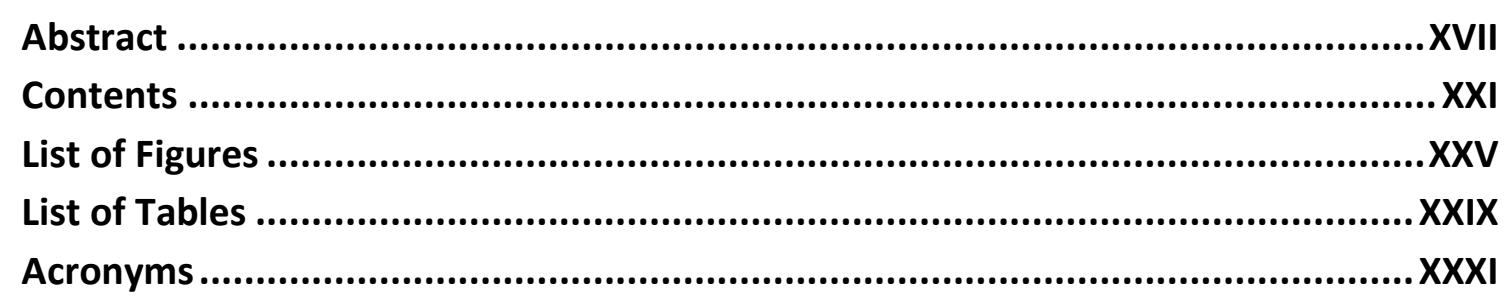

Chapter 1. Introduction ................................................................................ 1

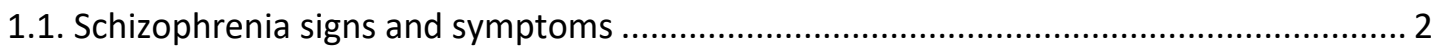

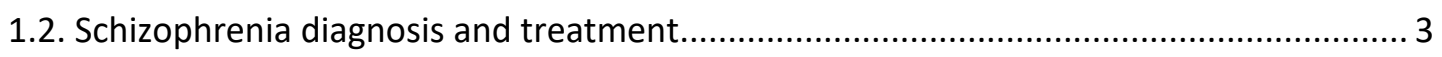

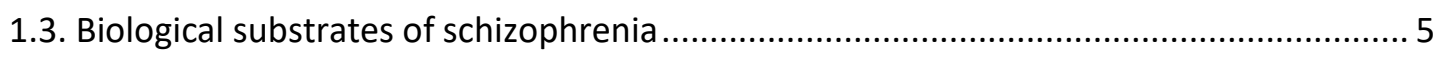

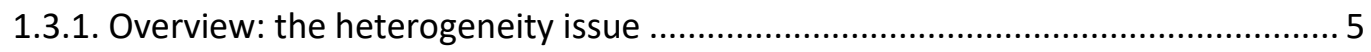

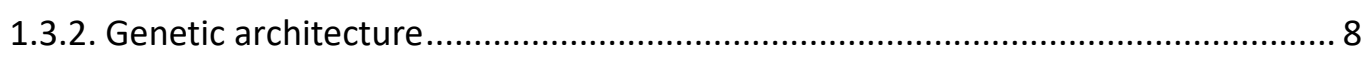

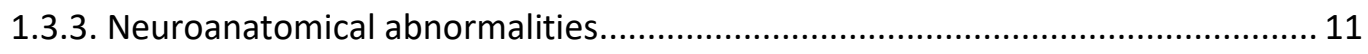

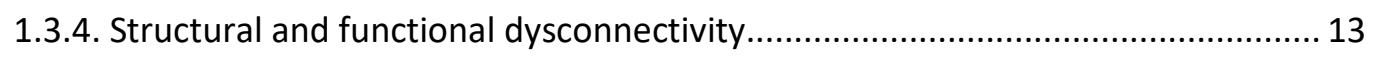

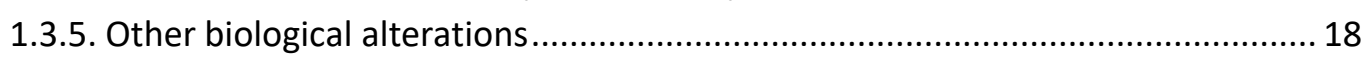

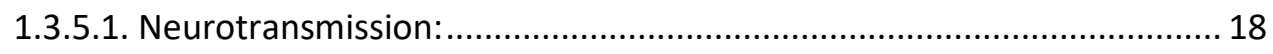

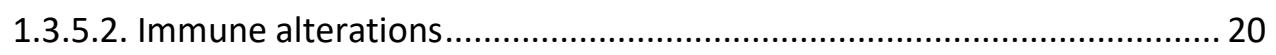

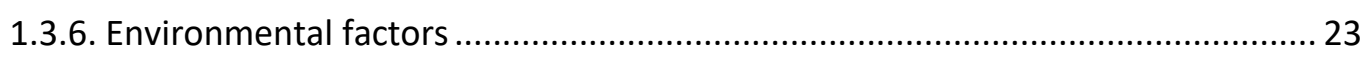

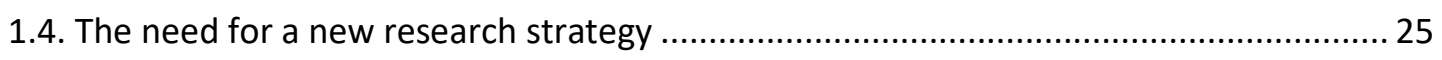

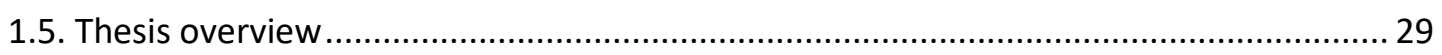

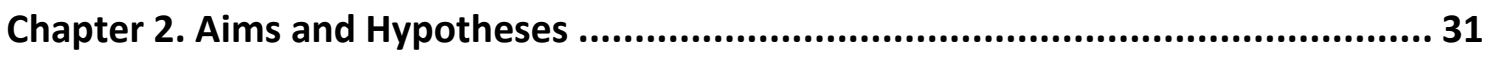

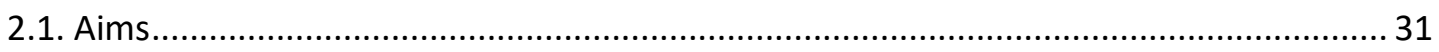

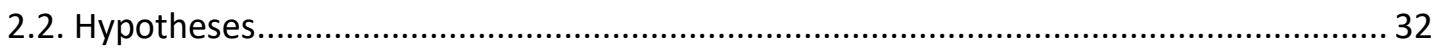

Chapter 3. Identification of two clusters within schizophrenia with different structural, functional and clinical characteristics.......................................................... 33

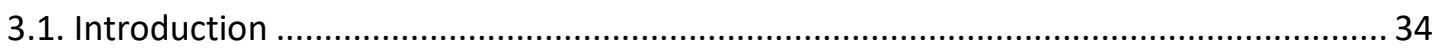

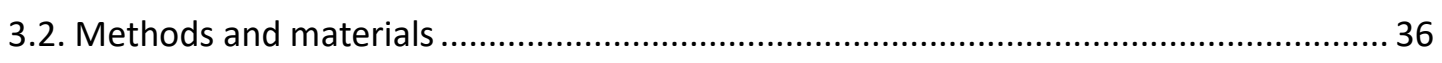

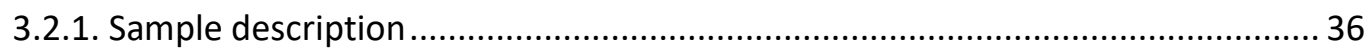

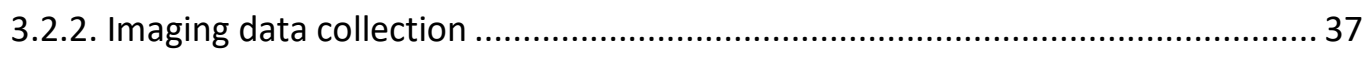

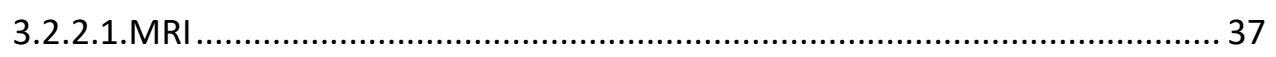

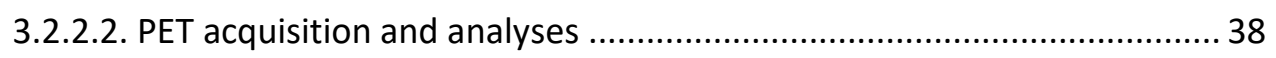

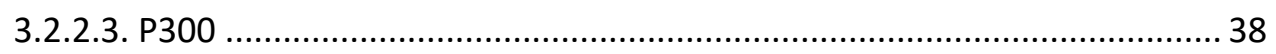

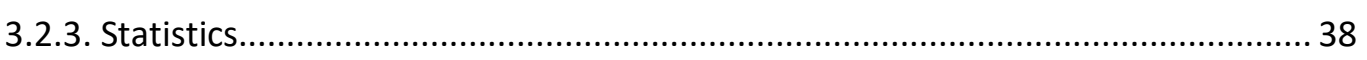

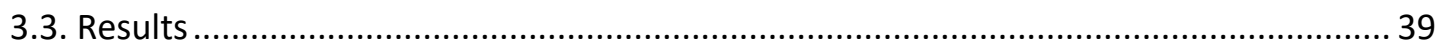

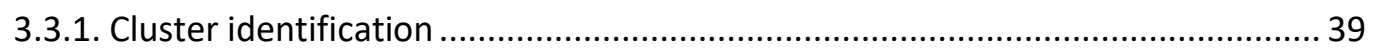

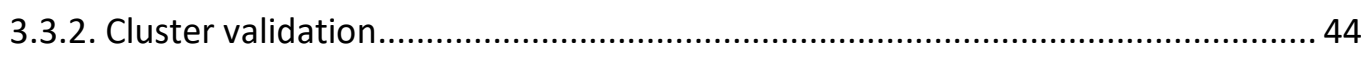

3.3.2.1. Comparison of MRI parameters between patient clusters and healthy controls. 


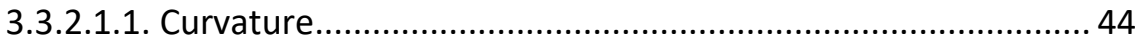

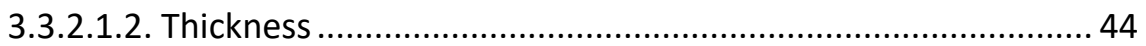

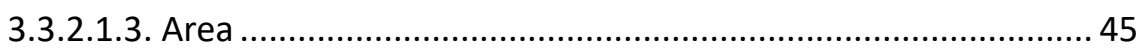

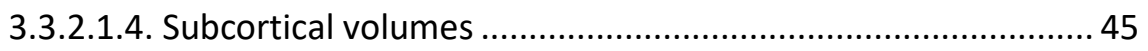

3.3.2.2. Comparison of other parameters between clusters of schizophrenia

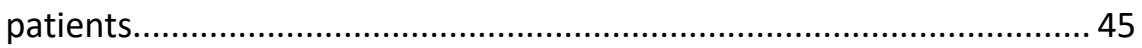

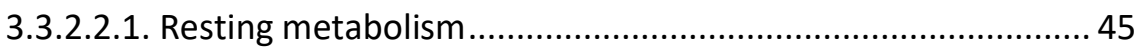

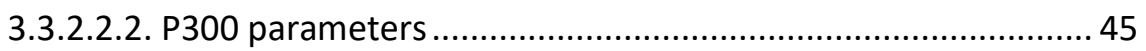

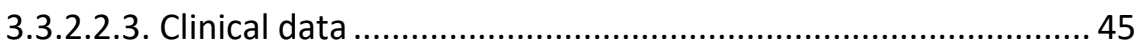

3.3.2.3. Comparison of other parameters between clusters of schizophrenia

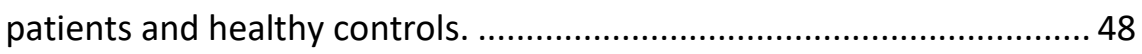

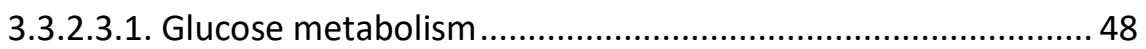

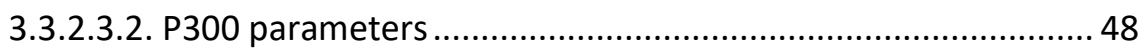

3.3.2.4. Association between curvature, metabolism and clinical variables ....... 49

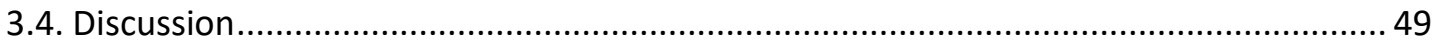

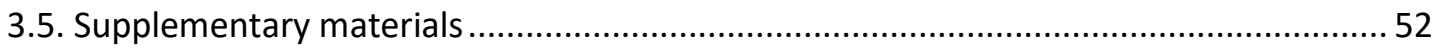

Chapter 4. Biological and cognitive correlates of cortical curvature in schizophrenia 55

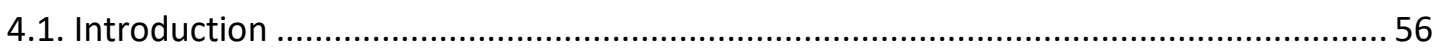

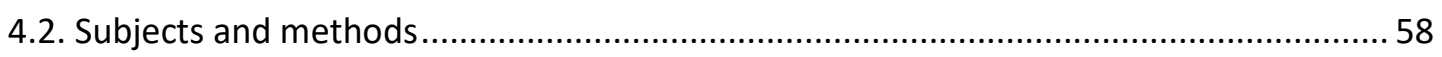

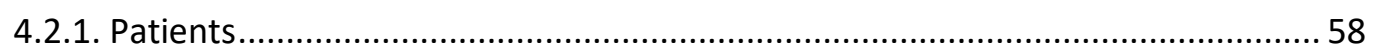

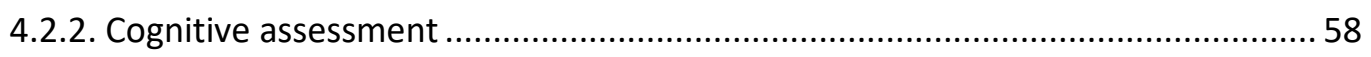

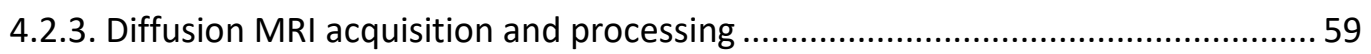

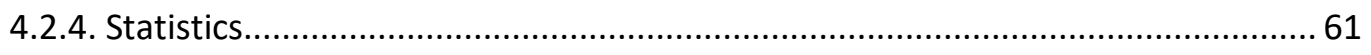

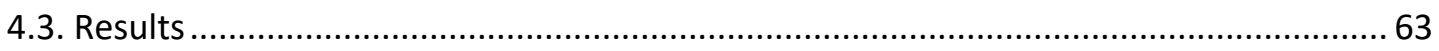

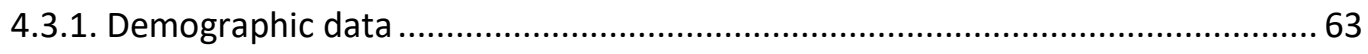

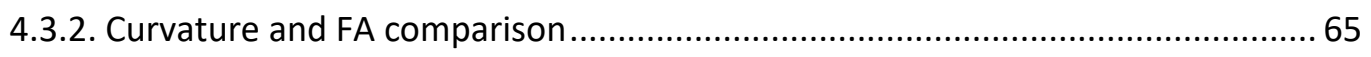

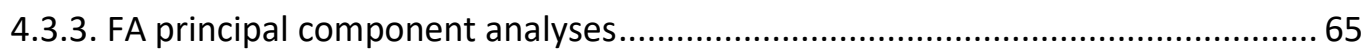

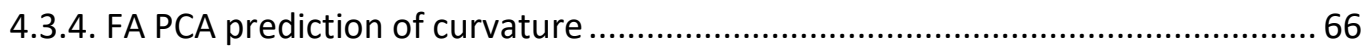

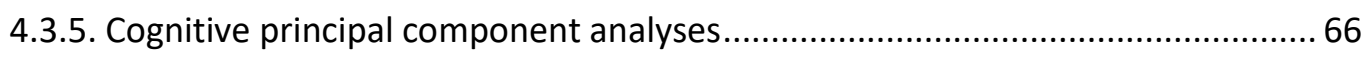

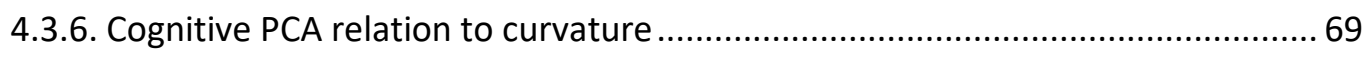

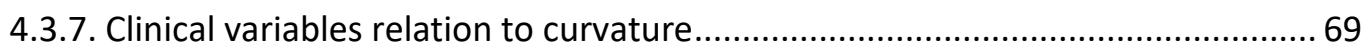

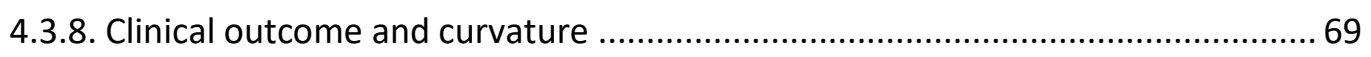

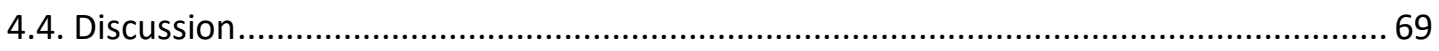

Chapter 5. Alterations in prefrontal connectivity in schizophrenia assessed using diffusion magnetic resonance imaging. ............................................................ 73

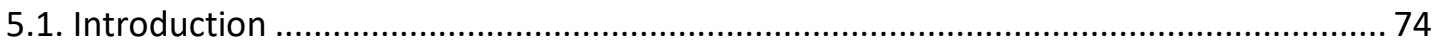

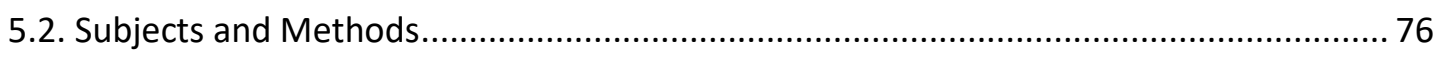

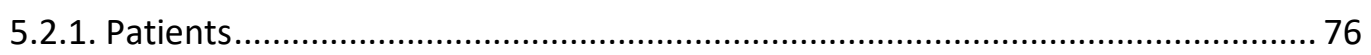

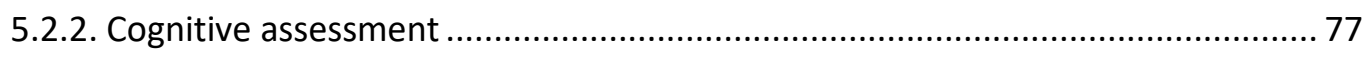

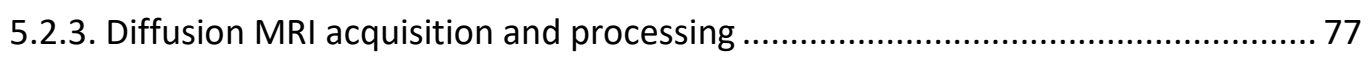

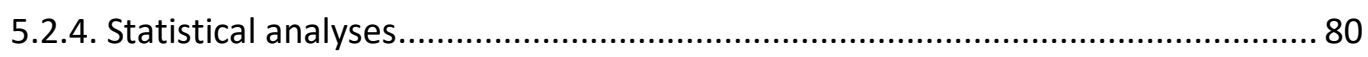

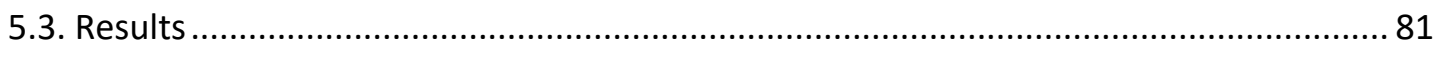

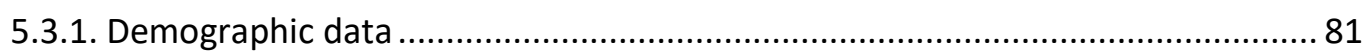




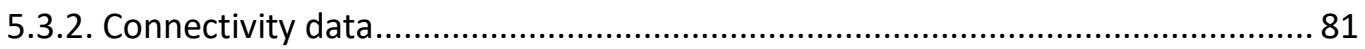

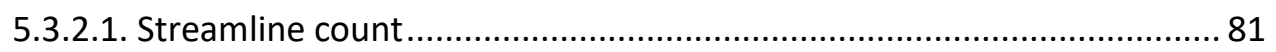

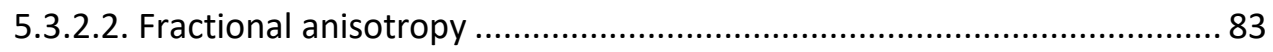

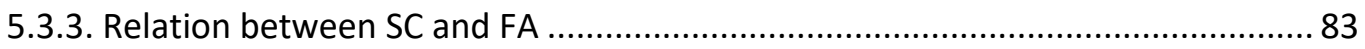

5.3.4. Relations of SC and FA with clinical and cognitive data ..................................... 83

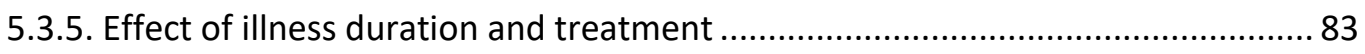

5.3.6. Comparison between first episode and controls ................................................. 85

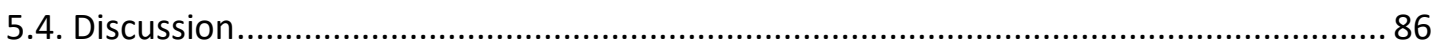

Chapter 6. Variation at NRG1 genotype related to modulation of small-world properties of the functional cortical network.................................................... 89

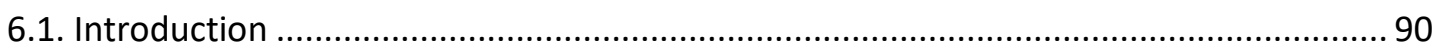

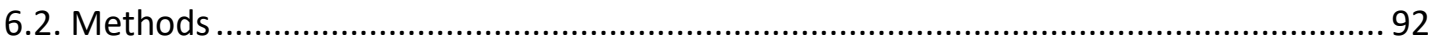

6.2.1. Participants: demographic and clinical assessment ........................................... 92

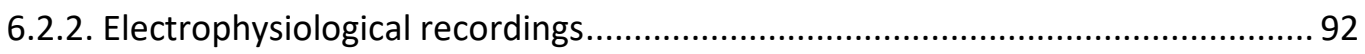

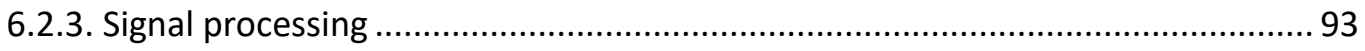

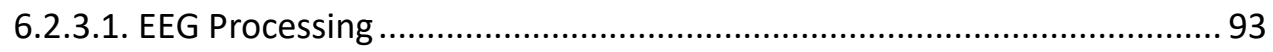

6.2.3.2. Continuous wavelet transform ............................................................ 93

6.2.3.3. Mean Squared Coherence Complex Network Theory ............................ 94

6.2.3.4. Complex Network Theory ................................................................... 94

6.2.3.5. Parameter baseline correction .............................................................. 95

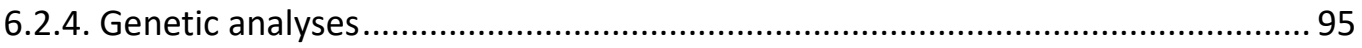

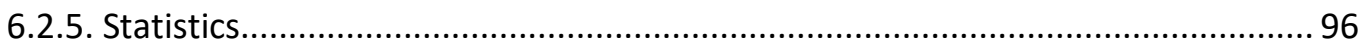

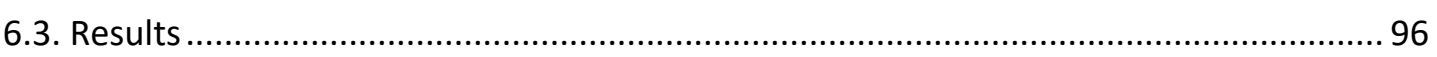

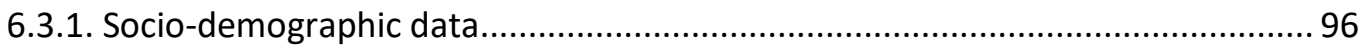

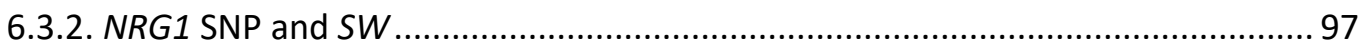

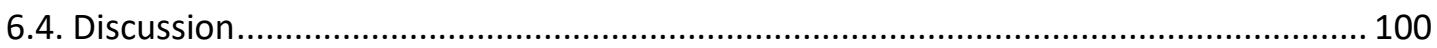

Chapter 7. Analysis of $K C N H 2$ and CACNA1C schizophrenia risk genes on EEG functional network modulation during an auditory odd-ball task ..................................... 103

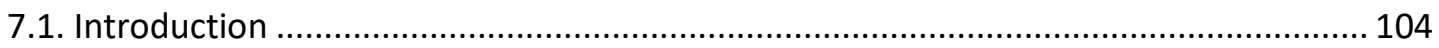

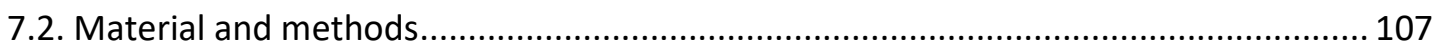

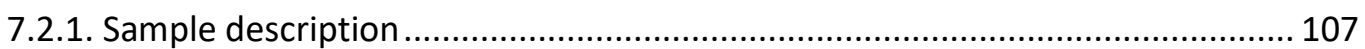

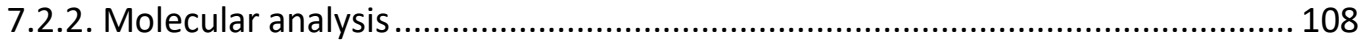

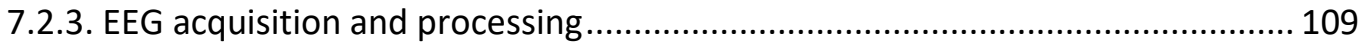

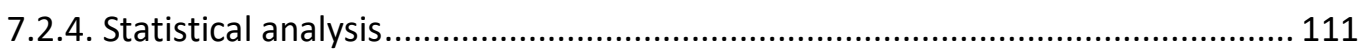

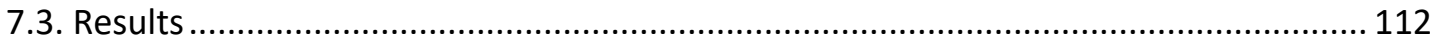

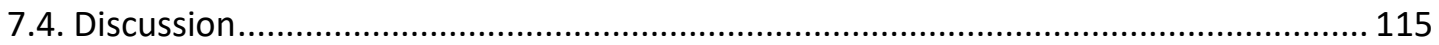

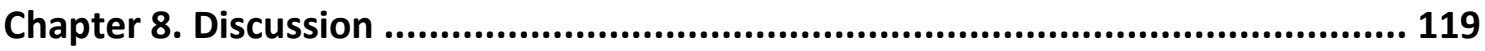

8.1. Subgroups of schizophrenia patients based on structural connectivity ....................... 122

8.2. Subgroups of schizophrenia patients based on genotype and functional connectivity 126

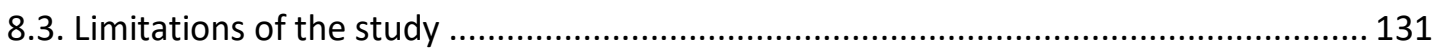


Chapter 9. Conclusions

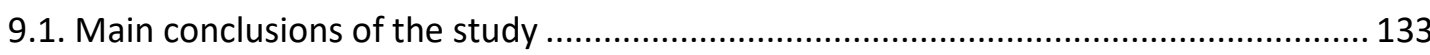

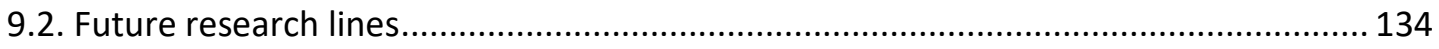

Appendix A. Student contributions to each publication ...................................... 137

Appendix B. Spanish summary / Resumen en castellano ................................... 139

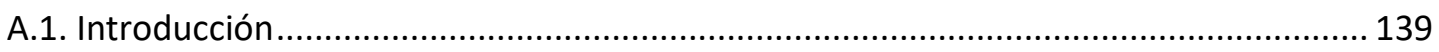

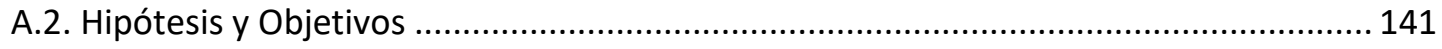

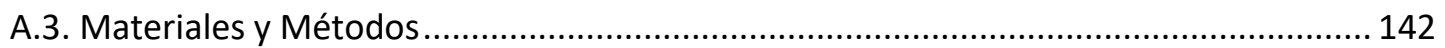

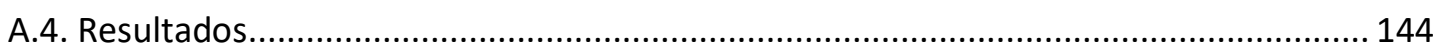

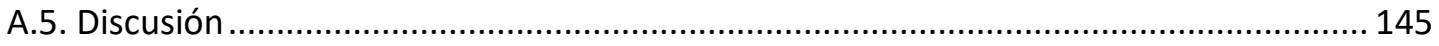

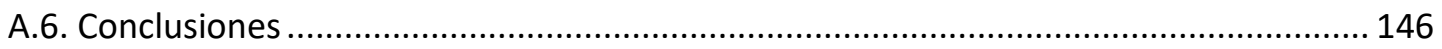

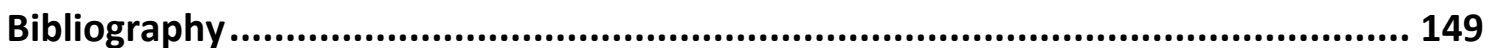




\section{List of Figures}

Figure 1.1. A scheme which integrates some of the risk factors and biological processes studied in relation to schizophrenia.

Figure 1.2. Resume of cytokines concentration in the serum of schizophrenia patients from meta-analytic studies. Different colours indicate different findings: $R e d=$ higher concentration in schizophrenia patients; Green = no difference between patients and controls; Blue = lower concentration in patients; White $=$ not analysed. FEP=first episode patients, $\mathrm{CHR}=$ chronic patients

Figure 1.3. Diagram of the possible disease scenarios from pathological processes to clinical manifestations: a) one pathological process produces one specific clinical manifestation; b) various pathological processes interact creating one clinical manifestation; c) different pathological processes produce specific clinical manifestations, although all clinical manifestations are grouped together as if they were only one specific manifestation; d) various pathological processes interact in different ways generating diverse clinical manifestations that, again, are grouped together as if they were one specific manifestation.

Figure 1.4. Comparison between typical research approaches in schizophrenia research and our suggested approach, which is useful to identify biologically significant subgroups within patients .28

Figure 3.1. Scatter plots of the distribution of the values of the first two principal components for curvature, thickness, area and subcortical volume, respectively. Subjects are identified by color code. Outliers are marked in red. In the lower row, Scatter plots for the three PCs of the total set of MRI markers, showing different symbols for the final cluster and the type of observation. In the three dimension scatter plot for these PCs, in figure 3.1e, the two clusters have been identified with different symbols, showing that patients identified primarily on the basis of higher mean curvature also showed globally smaller thickness and area. Moreover, in figure 3.1f, the observations have also been represented with different symbols for healthy controls, schizophrenia and bipolar disorder cases. . .40 
Figure 3.2. Distribution of factor scores for the PCA for curvature (PC1CURV), thickness (PC1THICK), and glucose metabolism (PC1GLMCR) in healthy controls (C), cluster A schizophrenia patients (SzA) and cluster B schizophrenia patients (SzB) ........42

Supplementary figure 3.1. Scatterplot for the three PCs of the total set of MRI markers excluding those magnetic resonance whose slice thickness were $3 \mathrm{~mm}$

Supplementary figure 3.2. Distribution of the LDF in the groups defined by the slice thickness values: 1.5, 2, 2.2 and 3, and pairwise comparisons using Tukey test, no differences were found

Figure 4.1. Location of the areas selected for cortical curvature (rostral lateral prefrontal (RLPF, in red) and superior medial prefrontal (SMPF, in blue)) and fractional anisotropy (tracts: RLPF- Superior temporal gyrus (STG, in yellow). RLPF- Superior parietal cortex (SP, in green), RLPF-Anterior caudate cingulate cortex (ACC, in orange). SMPF-STG. SMPF-SP and RLPF-ACC) calculation .60

Figure 4.2. MRI processing pipeline applied to obtain cortical curvature and fractional anisotropy

Figure 4.3. Association graphs: a) associations between individual scores in the second factor of the principal component analysis of fractional anisotropy (PCA-F2; see text) and rostral lateral prefrontal (RLPF) curvature for all individuals, b) associations between PCA-F2 and SMPF for all individuals, and c) association between individual factor scores in the first component of cognitive performance PCA (printCG1, see text) and RLPF curvature for patients

Figure 5.1. Processing pipeline yielding streamline count and fractional anisotropy values 79

Figure 5.2. Anatomical location of the areas selected for the analyses .80

Figure 5.3. Scatterplots showing the significant associations between regional connectivity values and clinical scores. Black triangles = chronic patients, grey squares= first-episode patients

Figure 5.4. Scatterplots showing the significant associations between regional connectivity values and illness duration .85 
Figure 6.1. Distribution of significant $\mathrm{SWnMb}$ modulation values ( $\mathrm{SWnRb}-\mathrm{SWnBLb})$ in non-risk allele carriers and risk allele carriers

Figure 7.1. Patterns of SWn changes per group and genotype (KNCH2 rs3800779) from pre-stimulus to response windows. 114

Figure 7.2. Distribution of $\mathrm{SWn}$ values at the pre-stimulus window (left) and modulation (right). Significant differences are marked with asterisk: ${ }^{*} p<0.05 ;{ }^{* *} p<0.01$. Mean and standard deviation for each group and genotype ( $\mathrm{KCNH} 2$ rs3800779) are in the bottom of the figure.

Figure 8.1. A scheme summarizing the most important findings in this thesis .122 



\section{List of Tables}

Table 3.1. Demographic, clinical, electrophysiological, and principal component values. Values are shown as means with standard deviation, SD, in brackets. Significance differences are shown with two types of symbols: ${ }^{*} p=0.08,{ }^{*} p=0.05,{ }^{*} p=0.01$ and ${ }^{* * *} p=0.001$ in comparison between schizophrenia clusters; ${ }^{\circ} p=0.05,{ }^{\circ} p=0.01$, ${ }^{\circ 00} p=0.001$ in comparison to healthy controls.

Table 3.2. Cortical curvature, thickness and area values for every cluster. The data are shown as means and standard deviation in brackets. Significance differences are shown with two types of symbols, each one with three categories: ${ }^{*} p=0.005,{ }^{* *} p=0.001$ and ${ }^{* * *} p=0.0001$ in comparison between schizophrenia clusters; ${ }^{\circ} p=0.005,{ }^{\circ \circ} p=0.001$, and ${ }^{000} p=0.0001$ in comparison to healthy controls

Table 3.3. Metabolic activity values. The data are shown as means and standard deviation in brackets. Significance differences are shown with two types of symbols, each one with three categories: ${ }^{*} p<0.005,{ }^{* *} p<0.001$ and ${ }^{* * *} p<0.0001$ in comparison between schizophrenia clusters; ${ }^{\circ} p<0.005,{ }^{\circ 0} p<0.001,{ }^{\circ 00} p<0.0001$ in comparison to healthy controls. .48

Supplementary table 3.1. Subcortical volumes values. The data are shown as mean and standard deviation in brackets. There were no significant differences in any comparison

Table 4.1. Demographic, clinic, cognitive, curvature and FA data are shown for patients, FE and controls. Significant differences with controls: ${ }^{*} p<0.05, * * p<0.01$, $* * * p=0.001$

Table 4.2. Rotated component matrix for fractional anisotropy PCA. Rotation method: Varimax and Kaiser normalization. Two main components were extracted ...65

Table 4.3. Regression coefficients (R2) between curvature values for the right lateral (RLPF) and superior medial (SMPF) prefrontal regions and the factor scores summarizing FA values respectively for the fronto-parietal and right fronto-temporal tracts (PCA-F1) and for fronto-cingulate and left RLPF-superior temporal tract (PCA-F2). Beta 
coefficients and $F$ values for the corresponding regression models are also shown. ${ }^{*} p<0.05 ;{ }^{* *} p<0.01 ; * * * p<0.001 ;$ n.s: non-significant

Table 4.4. Rotated component matrix for cognitive PCA. Rotation method: Varimax and Kaiser normalization. One main component was extracted

Table 5.1. Demographic and clinical data shown as mean (standard deviation). Firstepisode group ( $n=14)$ is a sub-sample of the schizophrenia patients group $(n=27) \ldots . .76$

Table 5.2. SC and FA values for each pair of regions, shown as mean (sd). The statistically significant differences between patients and healthy controls are highlighted: $* \mathrm{p}<0.05 ;{ }^{* *} \mathrm{p}<0.01 ;{ }^{* *} \mathrm{p}<0.001$

Table 6.1. Socio-demographic data and $\boldsymbol{S W n M b}$ values for the cohort of subjects enrolled in the study. Data was divided into two groups, which were defined depending on the presence of NRG1 risk polymorphisms for psychoses. SWn values were calculated as the difference between $\mathrm{SWn}$ at response and baseline windows ( $\boldsymbol{S W n R} \boldsymbol{R}$ $\boldsymbol{S W n B L b}$. Positive values represent a SW increase at response. Statistically significant differences between risk and no-risk allele carriers are marked with an asterisk: * $p<0.05$ .98

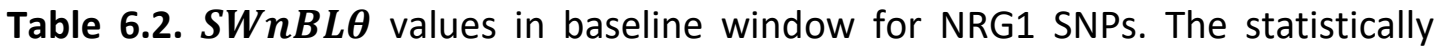
significant differences between risk alleles carriers and non-risk allele carriers are marked with asterisks: ${ }^{*} p<0.05 ;{ }^{* *} p<0.01 ;{ }^{* * *} p<0.001$

Table 7.1. Sociodemographic and clinical information of patients and controls. Statistically significant differences were detected only in relation to patients' years of education (in bold, $p<0.01$ ). .108

Table 7.2. Information on SNPs included in this study. The table includes the dbSNP number, the genomic and gene position and the alleles of each SNP (GRCh38.p7). Observed genotypic and allelic frequencies are also given.

Table 7.3. Sociodemographic, clinical and EEG functional connectivity variables mean and standard deviation per group and genotype. Differences between genotypic groups (risk-allele carriers vs non-carriers) within patients or controls are marked in bold $(p<0.01)$ 


\title{
Acronyms
}

\author{
A: $\quad$ Adenine \\ ACC: $\quad$ Anterior Cingulate Cortex \\ BACS: Brief Assessment in Cognition in Schizophrenia Scale \\ BDNF: Brain-derived Neurotrophic Factor \\ C4: $\quad$ Component 4 of complement \\ CACC: $\quad$ Caudal Anterior Cingulate Cortex \\ CACNA1C: Calcium voltage-gated channel subunit Alpha1C gene \\ CLC: $\quad$ Clustering Coefficient \\ CNS: $\quad$ Central Nervous system \\ COMT: Cathecol-O-Methyltransferase \\ CPZ: Chlorpromazine \\ CSF: $\quad$ Cerebrospinal Fluid \\ CWT: Continuous Wavelet Transform \\ DLPFC: Dorsolateral Prefrontal Cortex \\ DMN: Default Mode Network \\ dMRI: Diffusion Magnetic Resonance Image \\ DNA: Deoxyribonucleic Acid \\ DSM: Diagnostic and Statistical Manual of Mental Disorders \\ DTI: $\quad$ Diffusion Tensor Imaging \\ DWIs: Diffusion Weighted Images \\ E/I: $\quad$ Excitatory/Inhibitory balance \\ EEG: Electroencephatography \\ FA: $\quad$ Fractional Anysotropy \\ FE: $\quad$ First-episode \\ fMRI: $\quad$ Functional Magnetic Resonance Image \\ GABA: Gamma-aminobutyric Acid \\ GM: $\quad$ Grey Matter \\ GWAS: Genome-Wide Association Studies \\ ICA: Independent Component Analysis \\ IQ: Intelligence Quotient \\ KCNH2: $\quad$ Pore-forming subunit potassium voltage-gated channel, subfamily $\mathrm{H}$ member 2 \\ LDF: Linear Discriminant Function \\ MEG: Magnetoencephalography \\ MHC: $\quad$ Major Histocompatibility Complex \\ MRI: $\quad$ Magnetic Resonance Image \\ mRNA: Messenger Ribonucleotid acid \\ MSC: $\quad$ Mean Squared Coherence
}


NMDAr: Glutamate receptor N-methyl-D-aspatate

NRG1: Neuregulin1

PANSS: Positive and Negative Syndrome Scale

PCA: $\quad$ Principal Component Analysis

PCR: Polymerase Chain Reaction

PCs: $\quad$ Principal Components

PET: $\quad$ Positron Emission Tomography

PFC: $\quad$ Prefrontal Cortex

PL: $\quad$ Path Length

RD: $\quad$ Radial Diffusivity

RLPF: $\quad$ Rostral Lateral Prefrontal Cortex

RNA: Ribonucleic Acid

ROI: Regions Of Interest

SC: $\quad$ Streamline Count

SMPF: $\quad$ Superior-medial Prefrontal Cortex

SNP: $\quad$ Single Nucleotide Polymorphism

SP: $\quad$ Superior Parietal Cortex

SPG: $\quad$ superior Parietal Gyrus

STG: $\quad$ Superior Temporal Gyrus

SW: Small-World

SzA: $\quad$ Cluster A Schizophrenia Patients

SzB: $\quad$ Cluster B Schizophrenia Patients

WAIS-III: Wechsler Adult Intelligence Scale

WCST: Wisconsin Card Sorting Test

WM: White Matter 


\section{Chapter 1}

\section{Introduction}

Schizophrenia is characterised by positive and negative symptoms as well as cognitive disturbances and presents an estimated lifetime prevalence of $0.3-0.7 \%$ and an incidence of 10.2-22.0 per 100,000 person-year ${ }^{1,2}$. Schizophrenia is one of the leading factors of global disease burden ${ }^{3}$ and it is largely associated with expenditure and economic losses. In Spain, the total cost of schizophrenia was estimated in 1,970 million euros in $2002,53 \%$ of which were direct costs, implying a $2.7 \%$ of the annual public health budget ${ }^{4}$.

Different theories aimed to explain the biological mechanisms underlying schizophrenia, however, they have managed to do so with a limited degree of success 5 . It seems likely that different processes such as genetic risk, neurodevelopmental alterations and environment hazards might, independently or not, contribute to the syndrome known as schizophrenia. Despite many decades of research in schizophrenia, huge efforts and investments, a perplexing lack of replication still exist across biological research results in this syndrome. This very low replication rate hampers our understanding of schizophrenia and, correspondingly, the development of better treatments. Therefore, some reframing may be needed in the research approach for this syndrome ${ }^{6}$.

The present thesis aims to study the possible heterogeneous biological underpinnings of schizophrenia by using structural and functional brain connectivity data in combination with genetic data. Overall, here we propose two different classification approaches to test the hypothesis that patients sharing a schizophrenia diagnosis would have different biological alterations. First, assessing the existence of subgroups of patients using anatomical data and data-driven classification analysis; and second, studying subgroups by using functional connectivity and genetical architecture data. 
This kind of approaches eventually allows classification of patients with schizophrenia into different biologically defined subgroups.

Taken as a whole, our results support the possibility of improving our understanding of the schizophrenia underpinnings through measurable biological impairments close to the mental functions altered in this syndrome (thoughts, emotions and behaviour). This thesis is supported by five articles, four of them already published in journals indexed in the Journal Citation Reports (JCR) and one recently submitted.

In the following sections, in order to justify the purpose of this thesis, we briefly summarize the main characteristics of schizophrenia, some of the biological mechanisms involved and the large heterogeneity observed among patients.

\subsection{Schizophrenia signs and symptoms}

The onset of schizophrenia usually happens in late adolescence or early adulthood (20-26 years old in males and 26-30 in females), preceded by a prodromal period with cognitive and behavioural alterations ${ }^{1}$. Life expectancy is 10-20 years lower in schizophrenia patients in comparison with the general population ${ }^{7}$, usually due to unhealthy lifestyles, treatment side effects, suicide, pulmonary and cardiovascular diseases ${ }^{8}$.

Schizophrenia signs and symptoms are usually grouped into three core types ${ }^{1,9,10}$ :

-Positive symptoms: mental and behavioural phenomena that should not be present in normal conditions, such as delusions, hallucinations and thought disturbances.

- Negative symptoms: lack of mental and behavioural phenomena that are expected to be present in normal conditions, such as a minimal amount of verbal interaction, impaired emotional reactivity, low social involvement or a lack of interest and enjoyment of activities.

-Cognitive symptoms: alterations in cognitive functions such as impaired memory, executive functioning, problem-solving or attention.

Although the alterations described above are common in schizophrenia, a large comorbidity of symptoms exists across psychiatric diagnostics ${ }^{1,11}$. For example, core 
symptoms of schizophrenia, such as hallucinations and delusions, can also be present in other psychiatric pathologies such as bipolar disorder, major depression or substanceinduced psychosis. On the other hand, characteristic symptoms of other mental disorders, such as apathy or sleep disturbances associated with major depression, are frequently found in schizophrenia patients. Additionally, it is worth noting that affective symptoms, which are characteristic of affective psychosis, are not included in current schizophrenia diagnostic criteria, even though they are present in a significant percentage of schizophrenia patients.

In conclusion, the large variability in symptoms within schizophrenia and, on the other hand, the similarities between this and other mental diseases make the characterisation of these clinical entities rather unspecific ${ }^{6}$.

\subsection{Schizophrenia diagnosis and treatment}

Two systems are worldwide used to diagnose schizophrenia: the Diagnostic and Statistical Manual of Mental Disorders (DSM) and the International Classification of Diseases (ICD). The former is published by the American Psychiatric Association (APA), currently under its 5 th edition (DSM-V) ${ }^{9}$, and the latter by the World Health Organization, now under its 10 th edition (the 11 th is underway) ${ }^{10}$.

This diagnosis is based on signs and symptoms according to self-reported experiences, clinical interviews and observations by the clinician. Nowadays, no reliable biological measurement is included in the schizophrenia diagnostic process due to the fact that results in the field have been repeatedly inconsistent so far, not reaching enough degree of sensitivity and specificity for that purpose.

According to DSM-V criteria, schizophrenia diagnosis could be reached if the patient has suffered at least two of the following symptoms for a month or longer: delusions, hallucinations, disorganized speech, catatonic or grossly disorganized behaviour, or negative symptoms like diminished emotional expression. One of them, at least, must be one of the first three. Moreover, the person should show a total period of disturbances of six months or longer, including possible residual and prodromal symptoms. Consequently, patient's work, social, or/and self-care functioning should be 
impaired for a diagnosis of schizophrenia. Finally, other types of psychosis such as mood disorders, psychosis induced by drugs or medical disorders should be ruled out.

Due to these broad criteria, patients sharing the schizophrenia diagnosis could largely differ in their clinical presentations. For example, one schizophrenia patient could show bizarre delusions causing distress and impairment for a period longer than 6 months, while another one could show negative symptoms and persistent disorganized speech and behaviour ${ }^{12}$

Furthermore, as it has been pointed out, core symptoms are shared to a large extent with other mental syndromes. Therefore, boundaries between diagnoses become blurred, hampering the diagnostic procedure.

Current treatments are mainly based on antipsychotic drugs, principally antagonists of dopamine D2 receptors although other approaches such as psychological therapies are also clinically useful ${ }^{1}$. Unfortunately, the response to antipsychotics varies enormously among patients. One-third of patients are estimated to be treatmentresistant ${ }^{13}$, associated with worst functioning and prognosis. Indeed, specific biological differences have been reported in non-responder patients, for example, more remarkable neuroanatomical alterations 14-16. Furthermore, higher glutamate abnormalities but normal dopamine levels have been found in these patients ${ }^{17,18}$, which may explain why D2 antagonists are not useful for them. Clozapine is the gold-standard antipsychotic for those treatment-resistant patients which, interestingly, has a relatively weak affinity and short-term occupation of dopamine receptors ${ }^{19,20}$.

Treatment strategies go beyond the current diagnosis. Antipsychotics and other drugs like antidepressants, anxiolytics or anticonvulsants are used for patients with psychotic features across psychiatric entities such as schizophrenia, bipolar disorder, substance-induced psychosis, major depression or, even Alzheimer's disease and other dementias (National Institute of mental health).

Prognosis is also largely variable among patients ${ }^{21}$. Some cases show a favourable outcome with long periods of remission or even no relapses and a good general function, while other patients have a devastating scenario with no complete remission and spending large periods in psychiatric wards ${ }^{9,22,23}$. Different research studies have tried 
to understand the causes of prognosis differences by analysing divergences in underlying mechanisms ${ }^{24,25}$, neuroanatomy or drug abuse ${ }^{26}$ without clear results. Among the more replicated predictors of good outcome are the presence of affective symptoms, shorter duration of untreated psychosis, rapid response to treatment, less negative symptom severity, or being female and employed ${ }^{27}$.

\subsection{Biological substrates of schizophrenia}

\subsubsection{Overview: the heterogeneity issue}

The aetiology of schizophrenia is largely unknown, without any concrete alterations known to underly the disorder. Previous efforts in search of the biological underpinnings of schizophrenia have yielded inconsistent results. Even the relatively more replicated findings, such as hyperdopaminergia or larger ventricular volumes, are insufficiently replicated to be accepted as schizophrenia substrates. A recent review analysed the possibility of using the most relevant research findings as potential biomarkers for the diagnosis of schizophrenia without success ${ }^{5}$.

Several biological mechanisms studied in relation to schizophrenia, such as the dopaminergic system or brain connectivity, have been found to be altered (figure 1.1). Nevertheless, research findings in this respect are largely heterogeneous and most have not been replicated in different samples. Indeed, meta-analyses and reviews highlight the large variability observed in all kinds of biological factors, making it difficult, or impossible, to reach global conclusions ${ }^{28-32}$ Importantly, although for certain variables patients show significant differences in comparison to controls, there is usually a large overlap in values between both samples.

Heterogeneity is accepted in terms of symptoms, response to treatment or outcome. Even in first-episodes, one of the most homogeneous samples (they share approximately the same age, illness duration and a low exposition to antipsychotics), a clear heterogeneity has been noted in both clinical manifestations and biological findings ${ }^{25,33-35}$. In addition, the biological mechanisms likely involved in schizophrenia are shared with other psychiatric conditions, which underscores their lack of clinical validity and specificity ${ }^{29,36-39}$. For example, genetic variants associated with the risk of 
schizophrenia are also implicated in an increased risk of suffering bipolar disorder, autism or major depression ${ }^{40-43}$.

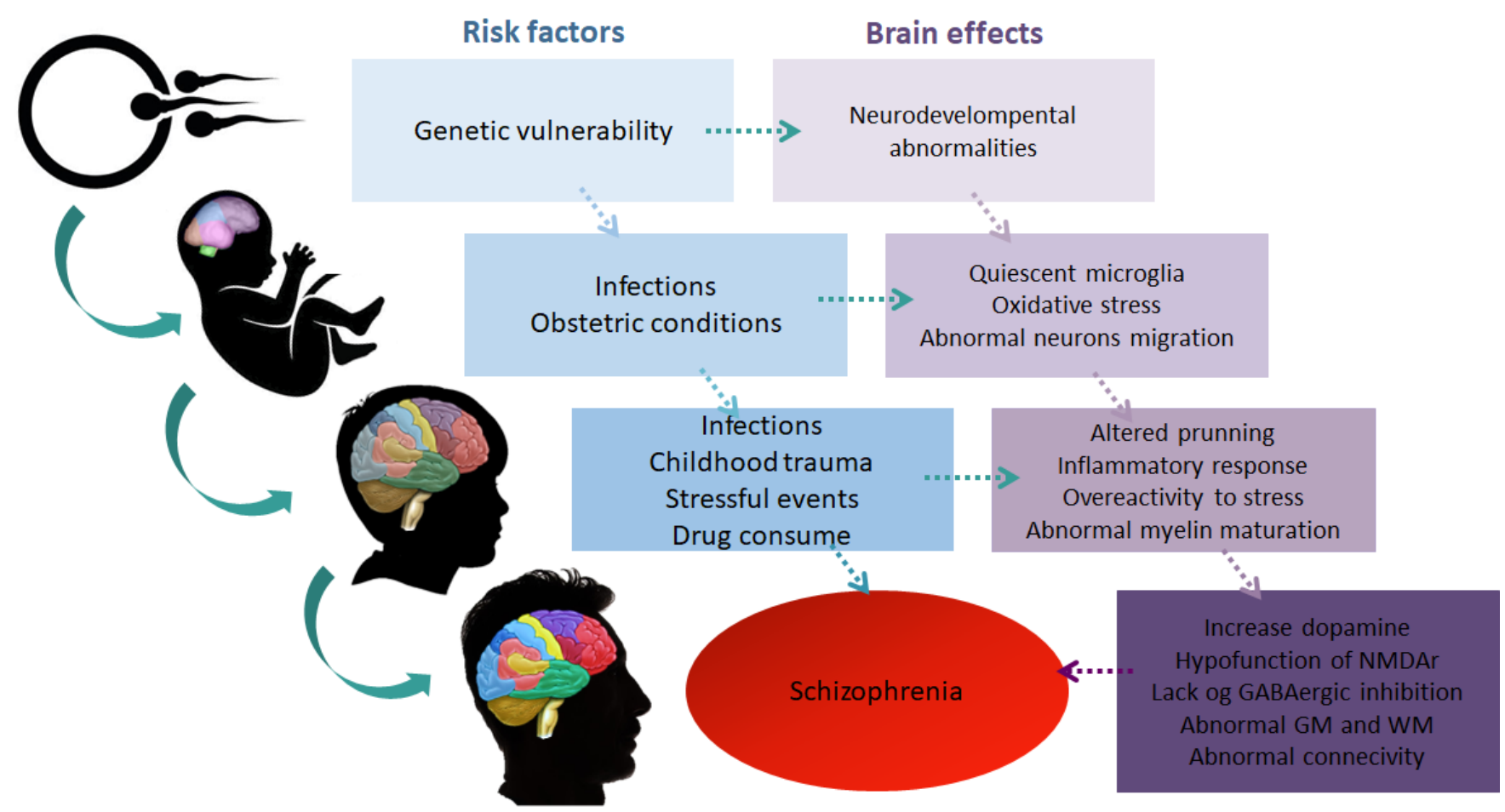

Figure 1.1. A scheme which integrates some of the risk factors and biological processes studied in relation to schizophrenia.

Different explanations could account for those heterogeneous research findings. First, one possibility could be that there are no biological processes underlying schizophrenia symptoms. However, this is implausible, since many findings have been partially replicated and drugs targeting specific neurotransmitter receptors are useful for a group of patients. Second, other option could be that all the disrupted systems studied in relation to schizophrenia, which would implicate many biological processes and brain regions, could converge to cause schizophrenia. This is an unlikely option, as it is not possible that so many alterations take place in a single person. Third, another explanation could be that the specific biological disruption explaining schizophrenia has not been found yet. This seems also a remote possibility since many different biological systems have been explored in large patient samples. Moreover, modern techniques, allowing a better examination of the central nervous system (CNS), add more complexity 
to the global picture rather than shedding light on the alterations. Fourth, the last possibility could be that schizophrenia is a syndrome encapsulating different abnormalities. This option seems to be more plausible, as heterogeneous results would be due to the intrinsic patients' biological variability, different patients' samples and recruiting strategies. Similar situations occur in other diseases where diagnostic procedures are complicated due to diverse clinical manifestations and trajectories, as in some types of cancer, for instance ${ }^{44}$.

Diagnostic manuals and research approaches comparing schizophrenia patients and controls contribute to the heterogeneity, since patients are classified exclusively based on symptoms and clinical signs. These manuals do not consider sources of patients' differences such as symptoms severity, outcome or response to treatment. Classification in terms of clinical differences has failed to lead us to the underlying neural pathologies. Moreover, clinical-based classification is unstable over time ${ }^{45}$. Therefore, regarding schizophrenia, current diagnostic tools may cluster together different syndromes, which precludes the possibility of finding disrupted mechanisms characterising them ${ }^{6}$.

Consequently, it seems plausible that schizophrenia diagnosis includes patients with different biological alterations contributing to interindividual variability in clinical manifestations. Classifying together different patients can lead to draw misleading conclusions. For instance, supposing no abnormalities in one biological process when patients' global mean for a variable is not significantly different from the mean in controls, although this variable is truly different in a subgroup of patients. On the other hand, assuming an altered process in all patients when patients' mean significantly differs from controls although the alteration is limited to one subgroup that strongly drives the differences in mean values.

Therefore, the typical research approach (i.e. comparing schizophrenia patients as a homogeneous group and healthy controls) could hamper the possibility of identifying reliable markers for some biologically plausible subgroups within this syndrome. Thus, a different approach is necessary for a real progress in identifying the underpinnings of schizophrenia. A good start point could be to take an example from another field of Medicine: 
Heart failure is a syndrome manifested by a group of symptoms. Each individual has a set of those possible clinical symptoms, meaning that each patient suffering heart failure syndrome could have a particular profile of symptoms. Nevertheless, despite sharing a heart failure syndrome diagnosis, the underlying causes can be diverse, such as myocarditis, myocardial ischemia or hypertension. Therefore, diagnosis cannot be reached based only on symptoms, rather auxiliary techniques are needed to identify the underlying causes. If these techniques are not available, we could wrongly group together patients with different aetiologies leading to inconclusive research results.

Similar to the heart failure syndrome, psychosis could be the common endpoint of different aetiologies. The current state of the art does not allow to diagnose schizophrenia patients based on their biological causes, but classifies all patients together, possibly hiding their biological differences. Thus, from a research perspective, it seems necessary to "deconstruct schizophrenia" in its multiple components ${ }^{11}$. Approaching clinical manifestations from a biological perspective will help in finding new biological valid diagnosis which, in the end, could implement more efficient treatment strategies directed towards the real underlying causes.

As a summary, several biological alterations are likely involved in the pathogenesis of schizophrenia syndrome and some of them may be distinctly involved in different subgroups of patients. This thesis explores this idea by searching distinct subgroups of patients based on anatomical and genetic data. In the following paragraphs, the main biological systems which seem to be altered in the schizophrenia syndrome are briefly revised, emphasizing the evidence of results heterogeneity.

\subsubsection{Genetic architecture}

The estimated schizophrenia heritability varies from $33 \%$ to $67 \%$ in family studies ${ }^{46-}$ 48. Twins studies yield concordance rates for the heritability of liability to schizophrenia to approximately an $80 \%{ }^{49}$. Therefore, genetics has an important role in the disease, but non-genetic factors should also play an important role in this syndrome. Indeed, a study suggested that only $25 \%$ of the risk of schizophrenia is explained by genetic variants identified in massive analysis ${ }^{50}$. Thus, interactions between genetic architecture and environment are key to understand the disorder ${ }^{51}$. 
Large efforts have been devoted to understanding the genetic background of schizophrenia with rather disappointing results. Thousands of genetic variants, not pathogenic per se, seem to be involved and interacting in complex ways. Each of those variants has a small effect increasing the risk of schizophrenia and they are also present in the normal population ${ }^{48,52}$. Therefore, a combination of risk genes, with thousands of different possible combinations within each patient, seems to mediate the genetic effect on the disease phenotype.

Genome-wide association studies (GWAS) are hypothesis-free approaches that can shed light on the understanding of genetic contribution to diseases. GWAS consists in a massive genotyping technique able to analyse thousands of single nucleotide polymorphism (SNPs) along the whole genome. SNPs are genetic variants differing in only one nitrogenous base which is present in at least $1 \%$ of the population. They are the most common genetic variation among humans, there can be up to 10 million SNPs in one person's genome ${ }^{53}$. The SNPs effect varies depending on its location on coding or noncoding regions. For those SNPs located in coding regions, depending on the genetic variation position, it may or may not produce a significant change in the protein expression or function.

The largest GWAS in schizophrenia so far ${ }^{48}$ estimated that around 6,300-10,200 independent and mostly common SNPs with small effect contributed to schizophrenia, accounting for around a $32 \%$ of liability variance. The SNPs most robustly associated with schizophrenia risk belong to dopamine regulation (DRD2), glutamatergic neurotransmission (GRM3, GRIN2A, SRR, CLCN3, and GRIA1), neuronal calcium signalling (CACNA1C, CACNB2, and CACNA1I), immune pathways (major histocompatibility complex (MHC)), transcriptional regulatory proteins (ZNF804A), non-coding regulatory RNAs (miRNA-137), synaptic plasticity and functionality (KCTD13, CNTN4, PAK6) and other SNPs located on inter-genetic non-coding regions that could relate to gene expression regulation.

The genetic risk of schizophrenia not explained by SNPs could be related to other types of genetic variants such as copy number variants (CNV), rare variants of strong effect, de novo variants or epigenetics (briefly revised in section 1.3.6) ${ }^{54}$. Copy number variants are common or rare deletions or duplications of DNA fragments (longer than 50 
base-pairs) that can affect various genes. For example, a deletion in the chromosome 3 (3q29) of approximately 1.6 megabases, including genes related to synapsis, dendritic spines or glutamate transmission, was related to a 40 -fold increase of schizophrenia risk 55-57.

Although genetic studies have expanded the knowledge about possible genetic risk factors in schizophrenia, some of the results are inconsistent. Some polymorphisms associated with schizophrenia in GWAS have not been replicated in smaller samples and, inversely, some candidate gene studies have not been replicated in GWAS. It seems impossible that the large amount of risk genetic variants, between six and ten thousand, combine in a similar way in all patients ${ }^{6,58}$. To complicate even more the genetic perspective, bipolar disorder, depression or intellectual disabilities share a large number of risk variants with schizophrenia, emphasizing the lack of genetic specificity and the similarities across categorical disorders ${ }^{40-43}$. The Brainstorm Consortium has recently published an interesting article based on GWAS meta-analysis showing the high genetic overlapping among many psychiatric diseases, including attention deficit, hyperactivity disorder, anorexia, anxiety disorders, autism, bipolar disorder, major depressive disorder, obsessive-compulsive disorder, post-traumatic stress, Tourette syndrome and schizophrenia ${ }^{59}$.

Interestingly, Arnedo and colleagues described different genetic networks (groups of interacting SNPs) associated with $70 \%$ of the risk of schizophrenia that might specifically lead to diverse phenotypes within "the schizophrenias" 58. They propose that schizophrenia includes different clinical syndromes related to specific sets of interacting risk genes and argue for assessing the genotype in a context of phenotypes described in detail, beyond the clinical diagnosis.

Thus, an alternative genetic approach, starting from genetical differences within patients to reach phenotype syndrome, may be useful instead of the classical genetic one (from diagnosis to genetics). Some brain dysfunctions or molecular abnormalities can be considered endophenotypes, defined as quantitative parameters within biological systems associated with diseases and more closely related to genetics than the clinical manifestation ${ }^{60}$. Therefore, studying the genetic contribution to endophenotypes may help in identifying subgroups with biological and clinical validity. 
As an example, a risk variant close to microRNA-137 gene (codifies a key protein in gene expression regulation which is associated with the risk of schizophrenia) can predict an earlier age-at-onset of schizophrenia and was associated with disrupted white matter integrity, smaller hippocampus and larger ventricles in patients ${ }^{61}$.

In this line, the present thesis studied the effect of selected polymorphisms on functional connectivity in controls and patients. The corresponding articles can be found in chapter six and seven of this manuscript. We selected polymorphisms related to myelinisation, neuron electrical properties regulation and excitatory/inhibitory (E/I) balance. In particular, three SNPs in neuregulin 1 gene (NRG1; a pleiotropic protein that participates in neuron development, migration and plasticity, myelination and NMDA receptor signalling) ${ }^{62}$, one SNP in a gene which encodes a pore protein, alpha1C, of Ltype voltage-gated calcium channel (CACNA1C), and another SNP in a gene which encodes a pore-forming subunit of voltage-gated potassium channel subfamily $\mathrm{H}$ member 2 ( $\mathrm{KCNH} 2$ ), were genotyped. Then, controls and patients (in the second article) were classified as carriers or non-carriers of each risk allele, and the genetic effects on functional connectivity modulation during an electroencephalographic (EEG) odd-ball task were analysed.

\subsubsection{Neuroanatomical abnormalities}

Brain anatomical abnormalities have been identified in schizophrenia both in vivo using neuroimaging techniques, and in post-mortem samples using histological techniques. Enlarged ventricles ${ }^{63}$, grey matter (GM) deficits at prefrontal, anterior cingulate, thalamus and temporal regions, as well as white matter (WM) reductions are among the most replicated anatomical findings, but inconsistencies are very common $30,31,64-67$.

Anatomical alterations appear to be already present at initial stages of the disease or before the illness onset ${ }^{25,68,69}$. Meta-analyses on longitudinal studies concluded that structural abnormalities occur already in prodromal stages, progressively worsening along the illness course, although it is likely some effect of treatment in this declining ${ }^{70-}$ 72 . 
The brain's macroanatomical changes could be partially explained at a microscale level by cellular and histological disruptions like altered neuropil density ${ }^{73}$, glial cells abnormalities, changes in neuron number, synaptic loss, abnormal axonal arborisation or altered neuronal pruning ${ }^{74-79}$.

Brain structural patterns in schizophrenia are very heterogeneous and even contradictory ${ }^{30,31}$. A recent meta-analysis ${ }^{28}$ analysed the variability in brain volume data (data dispersion) in first-episode schizophrenia (FES) patients and healthy controls. The authors collected regional brain volumetric measures from previous studies and calculated the inter-individual variability of that measures in each group. Subsequently, they compared the variability rates between groups. They observed that most measurements, except for the anterior cingulate cortex (ACC), showed greater variability (more dispersion) in first-episode patients than in controls; that is, patients' volumetric data seemed to differ significantly between samples. Moreover, anatomical values in some patients were in the same range than controls.

Based on heterogeneous literature findings, it has been suggested that distinctive patient subgroups within schizophrenia could differ on severity and extent of anatomical abnormalities, and that some alterations could even be specific of one particular subgroup of patients ${ }^{14}$. Indeed, the same research group observed brain structural differences within schizophrenia depending on genetics and symptoms. First, patients homozygous for rs1344706 polymorphism (AA) at ZNF804 gene, which codifies for a transcription factor, showed higher grey matter volumes mainly at the prefrontal cortex (PFC) and temporal cortex than heterozygous patients (AC) ${ }^{80}$. Interestingly, the opposite genetic effect was found in healthy controls, being the homozygous genotype (AA) associated with lower grey matter volumes than heterozygous genotype (AC). Secondly, they observed a widespread cortical thinning in a subgroup of schizophrenia patients with predominant negative symptoms, differing from the disorganised and paranoid symptoms subgroups ${ }^{81}$.

Other studies have identified brain anatomical differences among patients depending on their outcome or treatment response. Worse outcome schizophrenia patients presented GM deficits in subcortical areas particularly in thalamus and striatum in comparison to better outcome patients ${ }^{24}$. Another study in first-episode patients 
related prefrontal and medial-temporal alterations with worse one-year outcome ${ }^{25}$. Furthermore, treatment-resistant patients have been related to higher GM loss ${ }^{15,16,82}$. In schizophrenia patients with predominant negative symptoms, response to transcranial magnetic stimulation was predicted based on brain anatomical patterns. Specifically, marked GM reductions in prefrontal, temporal and cerebellar cortex and increases in parietal regions and thalamus were associated with non-response to active transcranial magnetic neurostimulation ${ }^{83}$.

As with other biological alterations, brain structural impairments associated with schizophrenia have also been identified in other psychiatric pathologies. Grey matter deficits in the dorsal anterior cingulate and anterior insula, related to executive function impairment, were concordant between schizophrenia, bipolar disorder, depression, addiction, obsessive-compulsive disorder and anxiety ${ }^{84}$. Moreover, other meta-analysis identified two trans-diagnostic subgroups based on GM alterations including schizophrenia, autism and obsessive-compulsive patients ${ }^{39}$. The first subgroup showed abnormalities in the insula, ventromedial PFC and thalamus, which were associated mainly with alterations in the self, introspection, emotions and salience. The second subgroup showed cortical abnormalities in the four lobes, middle insula and lingual areas which were related to alterations in motion, visual perception, face recognition, mentalizing and theory of mind ${ }^{39}$.

In general, anatomical findings support the idea that patients with schizophrenia do not share the same alterations and that some of these abnormalities are common to several psychiatric syndromes.

\subsubsection{Structural and functional dysconnectivity}

Higher mental functions are supported by the coordinated work of different brain areas ${ }^{85,86}$. Therefore, efficient communication between close and distant areas, also known as connectivity, is essential for proper mental functioning ${ }^{87}$. Cerebral connectivity allows integration of information between distant regions and communication between close regions in a specialized and segregated manner.

Three types of connectivity have been proposed ${ }^{87}$ : 
-Structural connectivity, defined as the anatomical connections formed by neuron synapsis, axons projections and myelin fibres. It can be assessed in post-mortem brains or in vivo using neuroimaging technics such as diffusion magnetic resonance (dMRI).

-Functional connectivity, defined as the statistical dependency or synchronization between neurophysiological signals in different brain locations. It could be measured in vivo using: i) electroencephalography (EEG), by measuring electrical oscillations created by groups of synchronously firing pyramidal neurons; ii) magnetoencephalography (MEG), by recording magnetic fields created by brain electrical currents, or ii) functional magnetic resonance (fMRI), which quantifies blood oxygen level dependent signal (BOLD), assuming that perfusion and oxygen consumption rise in active cerebral areas. EEG and MEG have a largest temporal resolution, but $\mathrm{fMRI}$ has a better spatial resolution.

-Effective connectivity, defined as the causal connections between areas or units, calculated with complex statistical models assessing the directionality and causality of connections ${ }^{88}$.

In 1998, Karl J. Friston formulated the disconnection hypothesis of schizophrenia ${ }^{89}$, proposing that impaired functional integration between neurons and brain areas is the central problem of schizophrenia. Nevertheless, not only functional but also structural connectivity seems to be disrupted. Olaf Sporns described schizophrenia as an altered structural connectivity disorder accompanied by disrupted functional communication between brain areas ${ }^{87}$. Therefore, assessing altered global connectivity (structural and functional) could help in elucidating pathogenic mechanisms within schizophrenia syndrome.

White matter connectivity deficits have been reported in schizophrenia in all disease stages, trending to worsen with illness duration. However, these findings are largely heterogeneous and inconclusive as it is highlighted in different reports ${ }^{66,90-92}$. Indeed, meta-analyses differ in localizing WM alterations $30,31,64,93$. The largest meta-analysis, as far as I know, including data from both volumetric WM (using volumetric brain morphology technique, VBM) and tractography (assessed with Diffusion Tensor Imaging, $\mathrm{DTI})$, located these alterations mainly in fronto-temporal-limbic, callosal-commissural and motor descending projections ${ }^{30}$. 
Regarding functional connectivity, both decreased and increased connectivity have been reported in schizophrenia at resting state and during task performance ${ }^{90,94-96}$. The default mode network (DMN) is a functional network more active in absence of specific cognitive tasks and likely associated with mind-wandering which includes the cingulate, medial PFC, medial temporal and inferior parietal cortex. This is one of the most studied functional networks at resting state in schizophrenia, with findings indicating both decreased ${ }^{97,98}$ and increased connectivity ${ }^{99-101}$ in first episode, chronic patients and their relatives. Also, a recent meta-analysis of resting state ${ }^{94}$ supports a lower connectivity in patients among areas of the following networks: affective, ventral attention, thalamus, somatosensory and DMN. These alterations seem to be already present in first-episode patients involving mostly language areas which may extend with illness progression ${ }^{102}$. During the performance of a P300 task (an event-related brain potential elicited by an infrequent target stimulus during an odd-ball paradigm), dysconnectivity was observed in first-episode and chronic patients involving mainly frontal, parietal, occipital, cingulate, and hippocampus regions ${ }^{103-105}$.

Interestingly, overlapping patterns of structural and functional connectivity have been found with other diseases. One meta-analysis described no WM integrity differences between schizophrenia and bipolar disorder ${ }^{106}$. Furthermore, both shared with major depression abnormal WM in the corpus callosum ${ }^{37}$. Also, similarities in functional disruptions were found between schizophrenia and autism ${ }^{107}$ and between schizophrenia, bipolar disorder and depression, especially in local functional connectivity during resting state ${ }^{108}$.

Graph theory ${ }^{87,109,110}$ is a useful method for assessing complex networks based on nodes and its connections, called edges. The application of graph theory to brain connectivity is useful to study global brain functions. According to available data, global brain connectivity, both structural and functional, has a small-world (SW) topology ${ }^{111-}$ 113. This type of network is characterised by the efficient transmission of information by means of balanced integration (long-distance communication) and segregation (local areas communication). SW organization has been found abnormal in patients with schizophrenia ${ }^{114-117}$. 
The structural connectome of patients with schizophrenia, in terms of graph theory, has been studied with different results. Structural connectivity has been described as less integrated, more segregated, with increased modularity and decreased global efficiency (usually measured using SW parameters) ${ }^{96,118-120}$. Moreover, rich-club nodes (key nodes for information transmission, highly connected between them and with the rest of the graph) organization and their connections seemed to be especially impaired in this syndrome ${ }^{121}$, likely affecting brain global communication ${ }^{122}$.

Functional networks in schizophrenia have also been studied using graph theory. Although again, discrepancies exist, the main findings during resting state pointed towards a functional network closer to random networks ${ }^{123}$ with decreased smallnetwork architecture. This implies a decreased network efficiency, normal or decreased integration and a tendency towards decreased segregation 95,123,124. During the performance of working memory tasks, patients also show worse, or even absent, smallnetwork architecture ${ }^{125}$, including heterogeneous results: decreased or increased clustering coefficient (CLC, a measure of segregation), and normal or longer path lengths (PL, a measure of integration) ${ }^{115,117,126}$. Functional SW disruptions have been found to be already present in high-risk individuals and first-episode patients 117,127 and, interestingly, they have also been observed in high functioning patients ${ }^{128}$. Moreover, similar to structural findings, functional connectivity in patients shows abnormalities in rich club hubs ${ }^{129}$ and lower connectivity among them ${ }^{95,122}$.

An unresolved question is how structural disconnectivity determines functional connectivity ${ }^{130}$. Based on research results, the direction of the alterations is not the same in both types of connectivity since main findings support both increased and decreased functional connectivity but only decreased structural connectivity. There are data supporting coupling 122,131,132 and decoupling 90,133 between structural and functional connectivity. Also, a recent meta-analysis found that, in drug-free patients, structural and functional abnormalities partially overlap within the default mode network and auditory networks ${ }^{134}$. On the contrary, we reported that both types of connectivity disruptions were not related, maybe because each type of alteration could be present in different subgroups of patients ${ }^{120}$. 
Connectivity differences have been observed within schizophrenia depending on clinical manifestations, supporting that connectivity alterations are not homogeneous among patients. Connections between the prefrontal and medial-temporal cortex to subcortical structures have been associated with patients outcome ${ }^{25}$, for example, patients with poor outcome showed decrease volume of the interior limb of the internal capsule (which was interpreted as decrease frontothalamic connectivity) in comparison with patients with good outcome. Also, first-episode patients who did not respond to treatment in the first year, had lower fractional anisotropy (FA), a measure of WM connections integrity (higher FA values is generally interpreted as better integrity), mainly at uncinate, cingulum and corpus callosum than responders patients and controls 135. In terms of functional connectivity, patients with verbal hallucinations, compared with patients without them, showed increased functional connectivity within DMN, but decreased connectivity within areas of the salience network ${ }^{136}$ (a functional network active when the individual is paying attention to salient stimuli, it is involved in detecting relevant stimuli and includes the dorsal ACC and insular cortex ${ }^{137}$ ). Treatment-resistant patients showed decreased connectivity in striato-nigro-striatal network in comparison to treatment-responders ${ }^{138}$. Unexpectedly, deficit schizophrenia patients (patients with primary and enduring negative symptoms ${ }^{139}$ ) showed increased functional integration (shorter connections between distant regions) and higher functional global efficiency in comparison to non-deficit patients 140

The effect of treatment on connectivity is not clear. Some antipsychotics seem to improve WM connectivity since increased FA (better WM integrity) was observed after one year of treatment ${ }^{135}$. Functional connectivity changes, mainly in cortico-striatal network, were described after treatment with risperidone or aripiprazole and increased connectivity was related to psychotic symptoms improvement ${ }^{141}$. Moreover, decreases of functional connectivity after 6 weeks of treatment with atypical antipsychotics ${ }^{142}$ or improved effective connectivity after 1 week of treatment ${ }^{143}$ were reported.

The above-mentioned evidence supports connectivity alterations, but the directions and localizations vary across studies. It could be possible that connectivity changes in different networks are caused by particular biological impairments, such as hypofunction of NMDA receptor, altered pruning or disrupted myelinisation in fibres 
with later maturation, and are related to different clinical manifestations ${ }^{111}$. Therefore, subgroups of patients within the same diagnosis may show different connectivity patterns related to specific disruptions of higher mental functions. However, all of them could fit into the current, biologically unspecific, schizophrenia criteria ${ }^{144}$.

In the present thesis, both structural and functional connectivity have been studied in schizophrenia in order to find patient subgroups with specific patrons of connectivity. First, brain cortical curvature was calculated as an indirect measure of structural connectivity. Second, DTI was included to understand the relation between cortical curvature and structural connectivity, measured by means of FA, as well as to assessed structural connectivity alterations in patients. Finally, EEG signals and graph-theory were used to assess functional connectivity in terms of small-world and its possible relation to genetic polymorphisms.

\subsubsection{Other biological alterations}

\subsubsection{Neurotransmission:}

Available evidence supports dopaminergic, glutamatergic and GABAergic signalling impairments in schizophrenia. Alterations in those systems are not mutually exclusive, since glutamate release is partially controlled by presynaptic dopamine receptors ${ }^{145}$, and GABA release is controlled by glutamate receptors in inhibitory neurons ${ }^{146,147}$. Moreover, genetic variants related to neurotransmission are associated with that syndrome ${ }^{48}$.

Dopamine transmission alterations have been related to schizophrenia $41,146,148$ on the basis of: i) the effectiveness of dopamine receptor D2 antagonists, ii) psychosis induced by dopamine agonists, and ii) higher dopamine receptor concentration in postmortem brain samples of schizophrenia patients. In its initial formulation, this theory proposed that positive symptoms would be caused by mesolimbic hyperdopaminergia, while negative and cognitive symptoms could be secondary to dopamine deficits in the frontal cortex. Antipsychotics would restore mesolimbic dopamine activity but would induce a further decrease in other dopamine pathways. Therefore, side effects would emerge as a consequence of the decreasing dopamine levels in nigrostriatal, 
mesocortical and tuberoinfundibular dopaminergic pathways ${ }^{145}$. Moreover, the altered dopamine levels could partially be due to impaired neurons phasic dopamine release to relevant and irrelevant stimuli ${ }^{149}$.

More recently, the dopamine hypothesis has been questioned, since recent studies showed that hyperdopaminergia is not present in all schizophrenia patients. Treatmentresistant patients may not show dysfunctional dopaminergic transmission 15,17. Interestingly, long-term treatment with antipsychotics has been related to up-regulation of the density of dopamine D2 receptors, associated with treatment resistance and psychotic exacerbation, i.e. dopamine super-sensitivity psychosis ${ }^{150}$. Thus, dopamine abnormalities do not seem to be homogeneous in schizophrenia patients but might characterize a subgroup of patients which respond to dopamine receptors blockers (current antipsychotics) ${ }^{145}$. Indeed, Howes and Kapur proposed a classification of patients based on dopaminergic levels, one group called "hyperdopaminergic" with elevated dopamine synthesis and release in the striatum, and another group called "normadopaminergic" without dopamine abnormalites ${ }^{151}$. Moreover, such dopamine level increase is not specific to schizophrenia as it has also been described in bipolar disorder or autism ${ }^{152}$.

Glutamate is the major excitatory neurotransmitter in the brain. The glutamatergic hypothesis proposed a hypofunction of the glutamate NMDA receptor in patients with schizophrenia ${ }^{153}$. NMDA receptor antagonists as ketamine or phencyclidine transiently mimic key psychotic symptoms of schizophrenia. Similarly, mouse models not expressing different NMDA receptor subunits (knockout models) exhibit schizophrenialike symptoms ${ }^{154,155}$. Different therapeutics approaches tried to study the beneficial effect of glutamate agonist but, unfortunately, with no conclusive results ${ }^{156}$.

Inhibitory interneurons releasing GABA (gamma-aminobutyric acid, the major inhibitory neurotransmitter) are key to control the neuronal firing tuning that supports complex cognitive processes. At the same time, interneurons firing is controlled by excitatory neurons, so a complex interaction between GABA and glutamate is necessary for a correct brain electrical tuning.

The NMDA receptor (NMDAr) hypofunction leads to a decreased activation of GABA inhibitory interneurons, creating an excitatory/inhibitory imbalance that disrupts the 
correct brain electrical synchrony. Thus, interneurons inefficiency might underlie gamma-frequency synchronization alterations ${ }^{157}$, higher electrical noise likely related to aberrant salience ${ }^{158,159}$, or alterations in functional connectivity ${ }^{160,161}$. More direct evidence of altered GABAergic signalling has been found in schizophrenia patients mainly supported by a decrease of glutamate decarboxylase 67 (GAD67) expression 162,163 , an important enzyme involved in the GABA synthesizing pathway.

As in the case of dopamine, glutamate and GABA dysfunction might differ in subgroups of patients with schizophrenia. Glutamatergic neurotransmission seems to be particularly dysfunctional in treatment-resistant patients $15,18,164,165$ with higher glutamatergic abnormalities apparently accompanied by normal striatal dopamine release ${ }^{18}$. In the same direction, mouse models of dopamine supersensitivity psychosis have shown a glutamatergic signalling decrease accompanied by an increase in GABAergic signalling in the striatum ${ }^{166}$.

\subsubsection{Immune alterations}

In recent years, the immune-inflammatory hypothesis of schizophrenia has gained relevance and visibility. This framework proposes that low-grade chronic inflammation could contribute to schizophrenia based on the following findings:

First, epidemiological studies have detected a high comorbidity between schizophrenia, autoimmune diseases and chronic inflammation ${ }^{167}$. Moreover, some autoimmune diseases produce schizophrenia-like symptoms or even could be initially confused with psychiatric diseases, as in the case of autoimmune encephalitis caused by anti-NMDA receptors antibodies ${ }^{168}$.

Second, prenatal or early childhood infections have been related to higher risk of schizophrenia and overactive inflammatory response in the adulthood 169-171. Nevertheless, most individuals exposed to infections during prenatal or early postnatal periods do not develop schizophrenia and most of the patients with schizophrenia did not suffer infections in that period ${ }^{171}$.

Third, some post-mortem studies have observed an increased microglial (the primary immune cells in the brain) density. Moreover, an increased microglial activation has been described both in vivo and in post-mortem samples ${ }^{172,173}$. Nevertheless, only half 
of the studies have replicated the findings in post-mortem studies ${ }^{32}$. In addition, in vivo PET studies to detect active microglia have yielded mainly negative results using secondgeneration radiotracers in spite of the positive findings observed with first-generation tracers ${ }^{32}$.

Fourth, elevated pro-inflammatory markers have been found both in the blood and the cerebrospinal fluid (CSF) of patients with schizophrenia. Intriguingly, meta-analyses reported contradictory results in the direction and type of cytokines abnormalities (figure 1.2) $34,38,174$.

\begin{tabular}{|c|c|c|c|c|c|c|c|}
\hline Meta-analyses & IL-6 & IL-1B & IFNg & TNFa & sIL-R2 & IL-2 & IL-4 \\
\hline \multicolumn{8}{|l|}{ FEP naïve, Miller et al., 2011} \\
\hline \multicolumn{8}{|c|}{ FEP naïve, Upthegrove et al., 2014} \\
\hline \multicolumn{8}{|l|}{ FEP naïve, Capuzzi et al., 2017} \\
\hline \multicolumn{8}{|c|}{ FEP treated, Goldsimith et al., 2016} \\
\hline \multicolumn{8}{|c|}{ FEP treated, Capuzzi et al., 2017} \\
\hline \multicolumn{8}{|c|}{ FEP early onset, Fraguas et al., 2017} \\
\hline \multicolumn{8}{|l|}{ CHR acute, Miller et al., 2011} \\
\hline \multicolumn{8}{|c|}{ CHR acute, Goldsmith et al., 2016} \\
\hline \multicolumn{8}{|l|}{ CHR, Potvin et al., 2008} \\
\hline \multicolumn{8}{|l|}{ CHR, Miller et al., 2011} \\
\hline \multicolumn{8}{|l|}{ CHR, Goldsmith et al., 2016} \\
\hline Not analysed & creas & & No di & nces & & crease & \\
\hline
\end{tabular}

Figure 1.2. Resume of cytokines concentration in the serum of schizophrenia patients from meta-analytic studies. Different colours indicate different findings: $R e d=$ higher concentration in schizophrenia patients; Green = no difference between patients and controls; Blue = lower concentration in patients; White $=$ not analysed. FEP=first episode patients, CHR= chronic patients.

Finally, GWAS studies have found that genetic areas related to immunity are strongly associated with the increased risk of schizophrenia, mainly the MHC region ${ }^{48}$. In particular, the strongest genetic association with schizophrenia was in component 4 of complement (C4) gene ${ }^{48,74}$. 
Inflammatory abnormalities have been related to processes deemed to be altered in schizophrenia ${ }^{169,175}$. Hyperactive microglia could produce an excess of neuronal pruning that could, in turn, lead to a decreased grey matter density. Indeed, Sekar and colleagues showed that schizophrenia-related polymorphisms in the $\mathrm{C} 4$ gene could raise $\mathrm{C} 4 \mathrm{~A}$ expression and also that higher C4A concentrations were associated with increased synaptic pruning in mouse models ${ }^{74}$. Overactive inflammatory responses could also damage oligodendrocytes and myelin, contributing to white matter impairments and therefore to structural connectivity alteration ${ }^{176}$. Finally, excessive chronic cytokines release and immune induction of tryptophan catabolites, such as kynurenine or kynurenic acid, may be related to NMDAr hypofunction and GABA release ${ }^{177}$.

However, as expected, immune abnormalities are not specific to schizophrenia. One meta-analysis observed similarities between schizophrenia, bipolar disorder and major depressive disorder in abnormal cytokine levels in CSF ${ }^{29}$. Some authors argued that antipsychotics can affect microglial activity and cytokines secretion ${ }^{42,178}$. In this sense, randomised controlled trials on the effects of anti-inflammatory drugs in co-treatment with antipsychotics described a large variety of results, from significant beneficial effects to irrelevant differences. These discrepancies could be due to inflammation changes through the illness course, or to immune abnormalities present only in a subgroup of patients. Therefore, immunomodulators may only have beneficial effects for a certain group of patients during a given stage of the disease ${ }^{174}$.

The described inconsistencies and some studies point out immune disturbances restricted to a subgroup of patients across diagnosis. Shannon Weickert and her group analysed inflammatory and stress-signalling markers in post-mortem brain tissues of schizophrenia, bipolar disorder patients and healthy controls ${ }^{179}$. They observed a distinguishable subgroup of patients, including cases with both diagnoses, characterised by higher levels of inflammatory and stress biomarkers. Besides, they also reported that those schizophrenia patients with higher inflammation markers also showed astrogliosis (increase in the number of astrocytes) ${ }^{180}$ and cortical grey matter reductions ${ }^{181}$. In a posterior work, including a different sample of patients with schizophrenia, the same group found a subgroup with increased inflammation, higher cytokines mRNA levels, lower verbal fluency scores and decreased Broca's area volume ${ }^{182}$. In the same 
direction, a subgroup of schizophrenia patients with an increase in either immune molecules or growth factors in serum, but not hormones, was also reported ${ }^{183}$.

\subsubsection{Environmental factors}

Some environmental variables have been associated with the increased risk of schizophrenia ${ }^{184,185}$. These factors might interact with genetic architecture, triggering the illness onset ${ }^{51}$. Environmental factors are especially relevant during sensitive time frames when the brain is under development and maturation, making prenatal, childhood and adolescence environments crucial for the individual health. Obstetric complications, migratory status to a racial or cultural minority, living in urban areas, birth in the winter season, drug abuse, trauma and stress are among the more replicated environmental risk factors for schizophrenia ${ }^{185-187}$. A meta-analysis in ultra high-risk individuals concluded that childhood trauma, adverse life events and affective impairments are those factors most associated with increased vulnerability to psychosis 184.

In general, early traumatic experiences are one of the leading environmental factors associated with an increase of schizophrenia risk, which could underlie migration and urbanicity risk factors. Migration status or belonging to a minority group has been related to discrimination, social adversities and isolation ${ }^{188,189}$. Moreover, people living in urban areas could suffer tougher social maladjustment, although it was also hypothesized that higher pollution or infection rates could mediate this relation to schizophrenia risk ${ }^{190-192}$. Interestingly, urbanicity was associated with a lower cortical thickness in healthy individuals ${ }^{193}$.

Early traumatic experiences, such as emotional, physical and sexual neglect or abuse, are more prevalent in people with schizophrenia ${ }^{194-196}$. Furthermore, childhood trauma has been related to more hospitalizations, worse functioning, lower quality of life and emotional distress in schizophrenia patients ${ }^{197}$. A recent meta-analysis supported the relationship between childhood trauma and severity of positive symptoms ${ }^{198}$. Trauma may lead to permanent changes during brain neurodevelopment, such as changes in the hypothalamic-pituitary-adrenal axis that sensitise individuals to posterior stressors and can even disrupt dopamine balance ${ }^{186,195}$. The interaction between childhood trauma 
and genetic variation could be the key to understanding its relation to schizophrenia. As an example, suffering childhood trauma plus carrying the risk allele of Val66Met polymorphisms in brain-derived neurotrophic factor (BDNF) gene had an additive effect, reducing BDNF mRNA levels and decreasing hippocampal volume ${ }^{51}$. Nevertheless, contradictory results can be found in the literature, for instance, no BDNF by childhood trauma interaction was observed on hippocampus volume and cognition ${ }^{199}$.

Another relevant environmental factor is drug consumption. Cannabis consumption, especially during adolescence, has a strong correlation with increased risk of schizophrenia and with an earlier age-at-onset ${ }^{200-202}$. Patients who consume cannabis have shown higher positive symptoms scores, worst psychosocial functioning, shorter time to relapse and worst general prognosis $26,197,203,204$. It has been suggested that cannabis could also be used by these patients as self-medication, in order to relieve primary psychotic symptoms or medication side-effects, improve self-esteem or overcome social isolation 204,205. Risk polymorphism in dopamine transporter 1 gene (DAT1) may mediate cannabis effects on abnormal hippocampal volumes 206. Furthermore, the modulation of the interaction between cannabis consumption and the risk of schizophrenia by the COMT gene has been widely studied. This gene codifies for an enzyme that degrades catecholamines such as dopamine. Val/Val genotype in the COMT Val158Met polymorphism could mediate the increased risk of schizophrenia associated with cannabis 207 and be related to an earlier psychosis onset 208 . Nevertheless, there are also contradictory and negative findings in the interaction between cannabis and COMT polymorphisms ${ }^{209-211}$.

Furthermore, dietary factors may also have a role in the disease, low levels of vitamin D or polyunsaturated fatty acids have been related to an increased schizophrenia risk 212,213 . Some studies pointed out that a diet supplementation with vitamin $D$ and polyunsaturated fatty acids could be beneficial on preventing and treating schizophrenia 214,215 .

The majority of the studies trying to understand environmental risk factors in schizophrenia are observational and epidemiological, hence it is not possible to infer the true causation of environmental factors. However, epigenetics could link environmental adversities to changes in gene expression and, therefore, to phenotypes 187,216,217. 
Epigenetic effects include: i) changes in DNA methylation profile, higher methylation rates of cytosines in $\mathrm{CpG}$ islands located on promoters which repress gene expression; ii) post-translational modifications of histones changing the chromatin status (heterochromatin, compacted chromatin and not accessible to transcriptional machinery; and euchromatin: less compacted chromatin and accessible for transcription); and iii) other regulatory mechanisms such as micro RNAs ${ }^{218}$. Interestingly, some epigenetic marks are also heritable in a process called imprinting 185,187 . Therefore, it is not only relevant how a child was raised but also the life events of their parents. The link between environmental situations and epigenetic changes can be addressed using animal models. Factors such as stress, hormones, maternal care or early infections have been associated with changes in epigenetic fingerprints ${ }^{216-218}$.

Finally, it is also important to consider that not all patients have been exposed to risk environmental situations. In addition, patients are exposed to different types of risk environmental factors, and most people exposed to similar situations do not develop schizophrenia. It seems likely that environmental factors exert their effect only when certain genetic risk is present ${ }^{209}$. Therefore, any environmental risk factor is not sufficient nor necessary by itself to develop schizophrenia.

\subsection{The need for a new research strategy}

As discussed above, the variety of aetiological factors associated with schizophrenia plausibly interact in a complex way leading to the clinical signs and symptoms of this syndrome $51,146,219$. It is not possible to know whether certain alterations are a consequence of other abnormalities or whether all of them are secondary to the same underlying disturbance or genetic background. Moreover, multiple biological processes and environmental hazards have been associated with increased schizophrenia risk (set of risk genes, different neurodevelopmental abnormalities, infections, trauma, stress, drug use, living style, etc.). This scenario creates thousands of possible combinations between genes, environment and biological processes. Thus, the probability that all possible scenarios occur in one's individual life is very low (figure 1.3, b), hence it is very unlikely that all patients suffer from the same process. It is then plausible that different 
combinations of genetic risk plus environmental factors lead to specific neural dysfunctions which take place in different groups of patients (figure 1.3, d). Despite the complexity of the possible scenarios and interactions between environmental and biological factors, current research approaches fail to take into account this complexity. Typical research strategies follow the idea that alterations converge in individuals with schizophrenia being the cause of the disease (figure 1.3, b). Nevertheless, the large clinical heterogeneity and the lack of research findings replication support that different and more complex scenarios are more likely (figure 1.3, c and d).
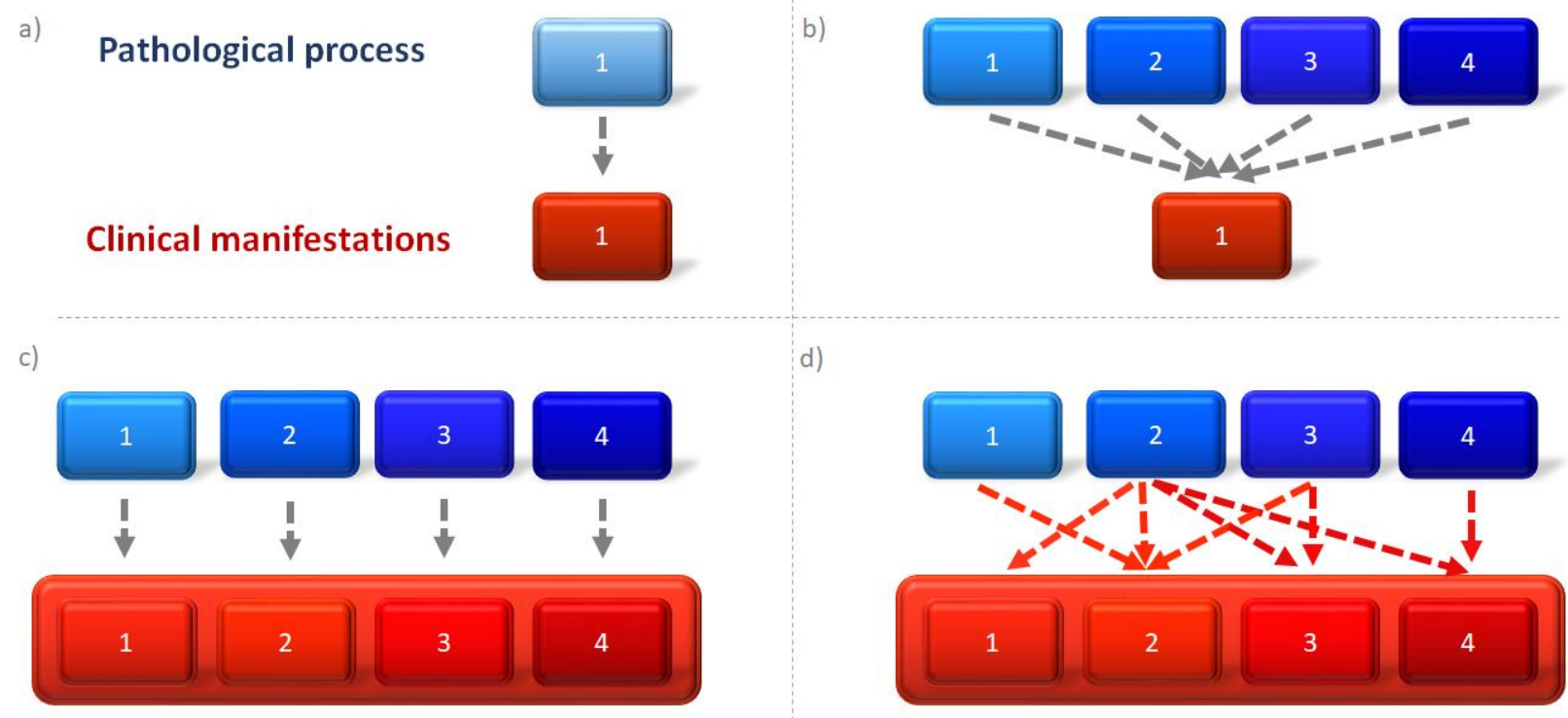

Figure 1.3. Diagram of the possible disease scenarios from pathological processes to clinical manifestations: a) one pathological process produces one specific clinical manifestation; b) various pathological processes interact creating one clinical manifestation; c) different pathological processes produce specific clinical manifestations, although all clinical manifestations are grouped together as if they were only one specific manifestation; d) various pathological processes interact in different ways generating diverse clinical manifestations that, again, are grouped together as if they were one specific manifestation. 
As a hypothetical example of the possibility presented in figure $1.3 \mathrm{~d}$, a group of patients with risk polymorphisms in genes involved in myelinisation and exposed to childhood trauma could show impaired myelinisation of fibres mainly connecting prefrontal cortex (which maturate later in life) and could be diagnosed with schizophrenia with predominant negative symptoms. Another group carrying risk polymorphisms involved in immune regulation and glutamate transmission and exposed to infectious processes or immune activation in early periods of life could show abnormalities in pruning processes. This would lead to impaired synaptic connections affecting functional networks integration and excessive microglial activations, damaging oligodendrocytes. These individuals might be diagnosed with schizophrenia with marked disorganization and cognitive deficits. A third group with a genetic background including risk polymorphisms related to dopaminergic signalling and exposed to obstetric complications might be diagnosed with schizophrenia with predominant positive symptoms and would respond to antipsychotic treatments. Currently, these three speculative scenarios with different aetiologies would produce symptoms according to current schizophrenia diagnostic criteria, and hence patients would be diagnosed and treated in a similar way. Also, if they participated in a research project, they would be included in the same group despite the fact that their aetiologies and symptoms were completely different (figure 1.3, d)).

It is important to note that the biological processes assessed so far are distant from the psychological functions known to be altered in schizophrenia such as thinking, behaviour and emotions. Thus, we may wonder: are we correctly designing research approaches? Given the lack of replicated findings, the answer may be negative. One potentially useful alternative to test is the hypothesis that groups of patients could be identified based on their common biological alterations, regardless of their clinical diagnosis. Therefore, new research strategies with techniques close to altered mental functions (i.e. connectivity is close to brain global functioning) are necessary to resolve this complex scenario. 

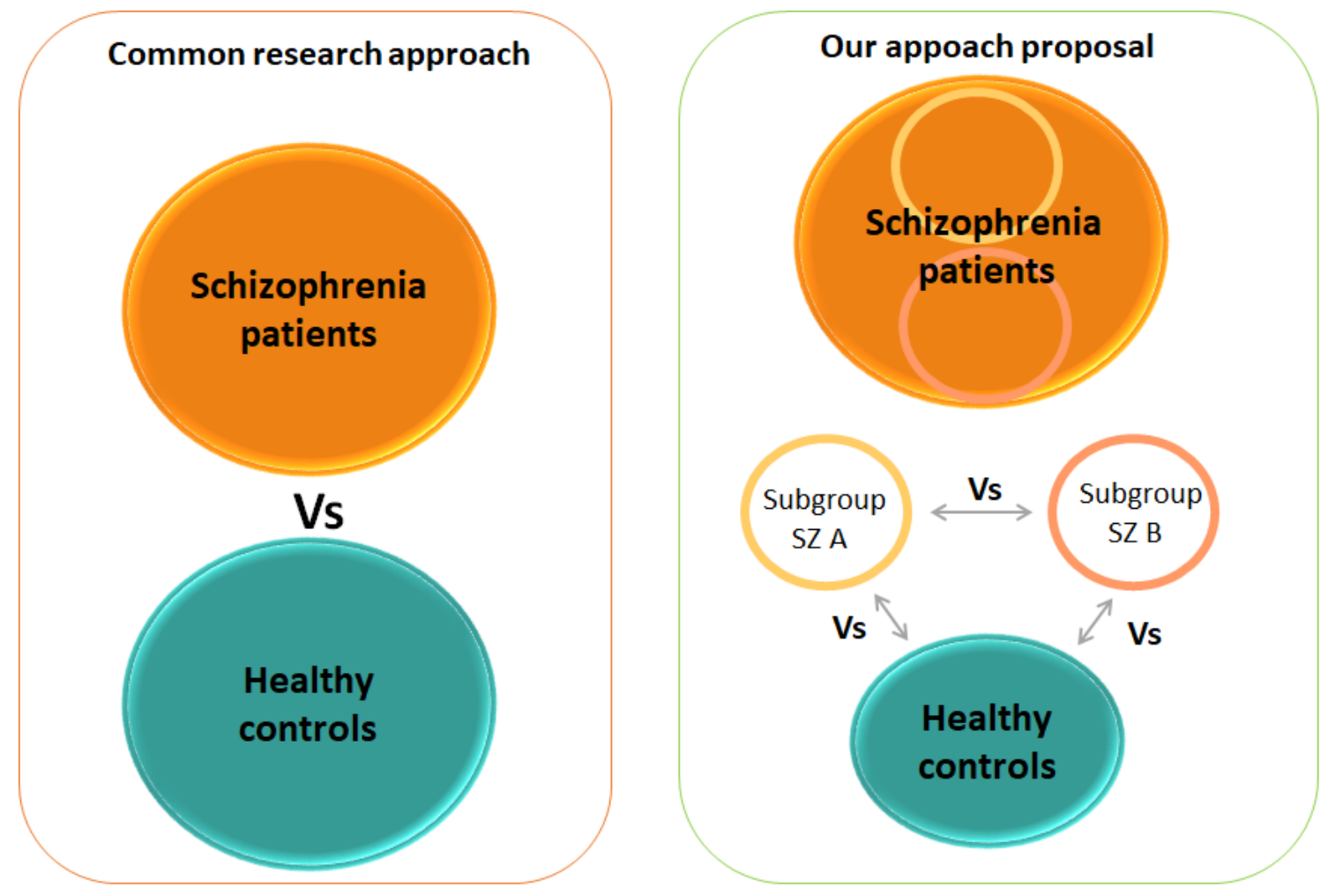

Figure 1.4. Comparison between typical research approaches in schizophrenia research and our suggested approach, which is useful to identify biologically significant subgroups within patients.

In this direction, some research programs have been launched aiming to explore different approaches, deal with the conundrum of schizophrenia cerebral underpinnings and find more accurate diagnostic tools. The Research Domain Criteria Initiative (RdoC), from the National Institute of Mental Health ${ }^{220,221}$, is."a research framework for new approaches to investigating mental disorders". This aims to collect a broad set of experimental data (i.e. genetical, psychological and neuroimaging) in large samples of patients with mental disorders to undercover valid behavioural and neurobiological dimensions. This would eventually help reframing the current diagnostic classifications of schizophrenia. A similar project is held by The Bipolar-Schizophrenia Network for Intermediate Phenotypes, a consortium built to identify psychosis subgroups by means of neurobiological biomarkers. In this line, our suggestions in this thesis are an unbiased data-driven research approach (figure 1.4) and genetic-based classification using connectivity measures. 


\subsection{Thesis overview}

The main purpose of this thesis is the search for subgroups of patients within schizophrenia based on structural/functional connectivity and genetic polymorphisms. The publications included addressed two classifying options, data-driven and geneticbased, and additionally aimed to understand the implications of the traits (gene polymorphisms and functional and structural connectivity) that characterise the different subgroups.

The thesis includes five articles, four of them already published and one recently submitted. The first one describes a data-driven approach defining a subgroup of patients based on structural data (mainly cortical curvature). The second article aimed to describe the relations between cortical gyrification and structural connectivity. In the third article, structural connectivity alterations in schizophrenia were described. The fourth and fifth studies analysed functional connectivity differences according to genetic polymorphism in controls and schizophrenia patients.

The thesis is organised into 9 chapters. This introduction is followed by the aims and hypotheses of the thesis, the five articles, a general discussion and conclusions. 


\section{Chapter 2}

\section{Aims and Hypotheses}

\subsection{Aims}

Following the ideas put forward in the introduction and based on the existing heterogeneity within schizophrenia and the importance of dysconnectivity to normal mental functioning, the following general and specific aims are proposed in this thesis:

\section{General aims:}

To use biological data in order to explore the existence of patient subgroups within schizophrenia, characterising the possible subgroups with other clinical and biological variables.

\section{Specific aims:}

1. To explore the existence of patient subgroups based on neuroanatomical data (cortical thickness, curvature, area and subcortical volume) using a statistical datadriven approach.

2. To validate subgroup of patients using clinical data (symptoms) and brain function measurements (electroencephalography and brain glucose metabolic rate).

3. To assess the relationship between frontal cortical curvature and structural connectivity quantified as fractional anisotropy from diffusion tensor imaging.

4. To evaluate frontal structural alterations in schizophrenia patients in comparison with healthy controls.

5. To assess whether genetic polymorphisms related to increased schizophrenia risk and involved in white matter development and excitatory/inhibitory balance contribute to differences in functional connectivity in healthy controls. 
6. To evaluate the possible influence of polymorphisms associated with schizophrenia risk, synaptic transmission and excitatory/inhibitory balance on functional connectivity in patients and healthy controls.

\subsection{Hypotheses}

\section{General hypothesis:}

Biological data and data-driven approaches would be useful to identify patient subgroups with common biological underpinnings within schizophrenia.

\section{Specific hypotheses:}

1. Subgroup of patients would be identified based on neuroanatomical data: cortical thickness, curvature, cortical surface area and subcortical volume.

2. Previously defined subgroups of patients would have differentiated clinical outcome and biological characteristics.

3. Frontal cortical curvature would be related to frontal structural connectivity.

4. Structural connectivity would be altered in schizophrenia in comparison to healthy controls.

5. Polymorphisms related to an increased risk of schizophrenia and implicated in white matter development and excitatory/inhibitory balance would have an effect on functional connectivity in healthy controls.

6. Polymorphisms associated with increased schizophrenia risk and implicated in synaptic transmission and excitatory/inhibitory balance would have a relevant effect on functional connectivity principally in patients. 


\section{Chapter 3}

\section{Identification of two clusters within}

\section{schizophrenia with different structural,}

\section{functional and clinical characteristics}

Published in: Progress in Neuro-Psychopharmacology and Biological Psychiatry 64, 79-86. doi: 10.1016/j.pnpbp.2015.06.015. ISSN 02785846.

Impact factor: 4.187 Neurosciences Q1 (63/259), Psychiatry Q1 (28/142).

Authors: Alba Lubeiro ${ }^{a}$, Cristina Rueda ${ }^{b}$, Juan A. Hernández ${ }^{c}$, Javier Sanz ${ }^{d}$, Fernando Sarramea ${ }^{\mathrm{e}}$, Vicente Molina ${ }^{\mathrm{a}, \mathrm{f}, \mathrm{g}, \mathrm{h}, ~ *}$
a. Department of Psychiatry, University of Valladolid, Valladolid, Spain.
b. Statistics Department, University of Valladolid, Valladolid, Spain.
c. University Rey Juan Carlos, Medical Imaging Laboratory, Madrid, Spain.
d. Department of Psychiatry, Hospital 12 de Octubre, Madrid, Spain.
e. Department of Psychiatry, Hospital Reina Sofía, Córdoba, Spain.
f. Psychiatry Service, Clinical University Hospital of Valladolid, Valladolid, Spain.
g. CIBERSAM (Biomedical Research Network: Mental Health; Instituto de Salud Carlos III).
h. Neurosciences Institute of Castilla y León (INCYL), University of Salamanca, Spain.
* Corresponding author 


\section{Abstract}

Several biologically distinct subgroups may coexist within schizophrenia, which may hamper the necessary replicability to translate research findings into clinical practice.

Cortical thickness, curvature and area values and subcortical volumes of 203 subjects (121 schizophrenia patients, out of which 64 were first episodes), 60 healthy controls and 22 bipolar patients were used to identify clusters using principal components and canonical discriminant analyses. Regional glucose metabolism using positron emission tomography, P300 event related potential, baseline clinical data and percentage of improvement with treatment were used to validate possible clusters based on MRI data.

All the controls, the bipolar patients and most of the schizophrenia patients were grouped in a cluster (cluster A). A group of 24 schizophrenia patients (12 first episodes), characterized by large intrinsic curvature values, was identified (cluster B). These patients, but not those in cluster A, showed reduced thalamic and cingulate glucose metabolism in comparison to controls, as well as a worsening of negative symptoms at follow-up. Patients in cluster A showed a significant putaminal metabolic increase, which was not observed for those in cluster B. P300 amplitude was reduced in patients of both clusters, in comparison to controls.

Results of this study support the existence of a biologically distinct group within the schizophrenia syndrome, characterized by increased cortical curvature values, reduced thalamic and cingulate metabolism, a lack of the expected increased putaminal metabolism with antipsychotics and persistent negative symptoms.

\subsection{Introduction}

More than 8000 genetic variants may contribute to the risk of suffering from schizophrenia ${ }^{48}$ and recent research supports schizophrenia subtypes characterized by both specific clinical presentation and clusters of genetic variants ${ }^{58}$. Considering the likely effect of genetic variation upon brain structure ${ }^{222}$, distinct groups within schizophrenia might be characterized by specific patterns of cerebral alterations. 
A possible approach to identify such groups is data-driven (a priori independently of diagnosis) cluster segregation, starting from plausible cerebral variables: i) previously associated with schizophrenia as a group but not unanimously replicated across samples, ii) not primarily related to confounders, such as treatment or chronicity, iii) with likely genetic influence, iv) associated with neurodevelopment and v) likely related to relevant clinical and biological variables.

Cortical thickness may prove useful to this purpose, since cortical thinning has been reported in first-episode (FE) and chronic schizophrenia patients ${ }^{223}$ (although not unanimously ${ }^{224}$ ) and in antipsychotic naïve patients ${ }^{225}$. It is highly heritable ${ }^{226}$, with a complex relation to genetic background in schizophrenia ${ }^{227}$ and early experiences 228 . Moreover, cortical thickness increases during normal neurodevelopment ${ }^{229}$ and has been associated with treatment response ${ }^{230}$ and cognition ${ }^{231}$ in FE patients.

Cortical curvature measures cortical folding and may reflect different neurobiological underpinnings ${ }^{232}$. It may contribute to the proposed clustering since higher ${ }^{233}$, normal 234 and reduced ${ }^{235}$ gyrification indexes have been reported in schizophrenia and $\mathrm{FE}$ patients have also shown altered gyrification ${ }^{236}$. Furthermore, gyrification has genetic underpinnings ${ }^{237}$ and cerebral gyrification takes place largely in the third trimester ${ }^{238}$, thus, altered gyrification may result from developmental events in this period. Finally, gyrification is associated with formation of proper cortico-cortical connections ${ }^{239}$.

The present study has reanalysed data from previous samples assessing cortical thickness and curvature in order to blindly investigate clustering of schizophrenia patients. Regional cortical area was also included in analyses, since it may convey different information than other structural data in schizophrenia ${ }^{240}$. In addition, subcortical volumes were included, given the caudate and thalamic volume association with poor-prognosis schizophrenia ${ }^{24}$. Regional cortical volumes were not included since they are largely explained by the corresponding area and thickness. In order to validate clusters, other data were collected, including clinical (baseline symptoms and variation with treatment) and biological (glucose metabolic rate, P300 amplitude and latency) parameters. Chronic bipolar subjects were introduced into the analyses to discard clusters being primarily related to chronicity and/or treatment. Therefore, rather than directly comparing anatomical values depending on clinical diagnosis (schizophrenia, 
bipolar and healthy controls), our approach was looking for biologically distinct clusters, not considering diagnosis a priori as the primary classifying factor. According to our hypothesis, clusters identified on the basis of structural alteration would be expected to be treatment-independent, associated with clinical outcome and/or presentation and associated with other relevant biological markers in order to be considered as valid.

\subsection{Methods and materials}

\subsubsection{Sample description}

Sample included 203 subjects: 121 schizophrenia (DSM-IV criteria), patients (64 patients were FE), 22 chronic bipolar patients and 60 healthy controls. Patients were recruited during a psychotic relapse (chronic patients) or first psychotic episode (FE), following their admission to a psychiatric short-term unit. After release they were treated and followed in an outpatient clinic.

MRI data were collected over a 15-year period, in the context of several research projects ( identification of cerebral correlates of treatment-resistance ${ }^{241}$, early stages ${ }^{242}$ of schizophrenia, differences between major psychoses ${ }^{243}$ and effects of antipsychotics on brain structure 244 .

18 chronic schizophrenia patients had been treated prior to inclusion with typical antipsychotics, and 39 with atypical antipsychotics. They were included the time of psychotic relapse. 18 bipolar patients were receiving atypical antipsychotics. FE patients had received antipsychotic treatment for less than three days prior to MRI.

Additional data used to validate the hypothesized clusters included:

- Resting metabolic glucose rates in dorsolateral prefrontal cortex (DLPFC), orbital, parietal, occipital and temporal lobes, cingulate, hippocampus, caudate, thalamus and pallidum were measured with F18-FDG PET during an attention test, in 64 schizophrenia patients (41 FE) and 34 healthy controls.

- $\quad$ P300 amplitude and latency were measured in 52 schizophrenia patients and 24 healthy controls. 
- $\quad$ Clinical status at inclusion was assessed using Positive/Negative Symptoms scale in schizophrenia (PANSS) ${ }^{245}$.

- Improvement percentage in 91 schizophrenia patients (40 FE) after 6 months follow-up.

Exclusion criteria included history of substance dependence, any current comorbid Axis I diagnosis or psychoactive treatment, serious head trauma or any neurological or systemic disorder with known effects on CNS.

After complete description of the study to the subjects, written informed consent was obtained. Full comprehension of the procedures was checked during the consent procedure. The research boards of the Hospitals of Salamanca and Doce de Octubre endorsed the study. The hospital ethical committee approved the study. All technics and procedures were conducted in accordance with the ethical standards of the Helsinki Declaration.

\subsubsection{Imaging data collection}

\subsubsection{MRI}

Description of MRI acquisition is available elsewhere ${ }^{241,243,244}$. In summary, 3D SPGR T1 MRI studies were acquired using a 1.5 system (159 cases using Philips Gyroscan and 44 using General Electrics 1.5 scanner). For each subject, a 3D T1 acquisition was obtained with following parameters: TR=7.5 ms, TE $=3.5 \mathrm{~ms}$, flip angle $=8 \stackrel{\circ}{0} 0.78 \times 0.78$, FOV $=240 \mathrm{~mm} \times 240 \mathrm{~mm}$, matrix size $=256 \times 256$, in 70-150 slices (thickness between $1.5 \mathrm{~mm}$ and $3 \mathrm{~mm})$.

Given their relevance in schizophrenia, the following regions were included in the initial clustering analysis (left and right separately): caudal anterior cingulate, caudal middle frontal, cuneus, inferior parietal, medial orbitofrontal, parahippocampal, pars orbitalis, pars triangularis, precentral, rostral anterior cingulate, rostral middle frontal, superior frontal, superior temporal and insula. For each of these regions, thickness, area and curvature were calculated using FreeSurfer (http://surfer.nmr.mgh.harvard.edu). In addition, subcortical volumes (pallidum, thalamus, caudate and hippocampus) were assessed. 


\subsubsection{PET acquisition and analyses}

Detailed description of acquisition and quantification is available elsewhere ${ }^{239,240}$. In summary, PET studies were obtained in SIEMENS Exact 47 tomography, 20 minutes after injecting $370 \mathrm{MBq}$ of $18 \mathrm{FDG}$ in resting condition. Image values were proportionally normalized to global count rate for each PET. To perform metabolic measurements of different brain structures, a two step procedure was adopted to co-register MRI and $\mathrm{PET}$, hence, defining regions of interest (ROI) onto each subject's Talairach co-ordinate system. ROI activity was calculated as the portion of tissue mask contained in the set of grid-cells defining the ROI.

\subsubsection{3. $\mathrm{P} 300$}

Details are given elsewhere ${ }^{246}$. A standard odd-ball paradigm was used and subjects were instructed to mentally count the number of target tones. Electroencephalograms were recorded from 16 scalp sites, according to 10/20 International System and analyses on P300 were performed on Pz site after correction of electrooculogram artifacts. The reference was algebraically-linked earlobes. The sampling rate was $250 \mathrm{~Hz}$.

\subsubsection{Statistics}

The analysis followed several stages in order to reduce data noise for the cluster search, since not all of MRI variables are useful to generate clusters and clusters may not differ in these data. In a first step, Principal Component Analysis (PCA) was repeatedly used to summarize information and to obtain simple graphical displays. The scores of the first principal components were used to identify outliers, to generate three initial groups, one with doubtful cases, and to describe the final clusters. The two initial components from this PCA for area, curvature, thickness and subcortical volumes were selected, yielding explained variability percentages: 55\%(PC1AREA)+4\%(PC2AREA) for area, 48\%(PC1CURV)+7\%(PC2CURV) for curvature, 54\%(PC1THICK)+7\%(PC2THICK) for thickness and 57\%(PC1SCVOL)+15\%(PC2SCVOL) for subcortical volume.

An algorithm based on canonical discriminant analysis was designed to classify doubtful cases. Once these cases were allocated to one of the two clusters, a linear discriminant function (LDF) was derived using the whole set of variables as a 
discrimination index for the clusters. A leave-one-out procedure was used to crossvalidate the classifier function.

Besides, a PCA for the glucose metabolic rate values was also carried out and the first principal component, PC1GLMCR (with percentages of explained variability of $34 \%$ ) was used to summarize metabolism information.

After having ascribed each subject to a given cluster, we looked for characterizing the schizophrenia groups included in these clusters. In order to do so, we compared anatomical, metabolic and electrophysiological values between patients in each cluster and healthy controls as well as between patients belonging to different clusters. Therefore, the differences in parameters used for cluster definition between patients in each cluster and healthy controls were assessed and other biological and clinical characteristics between patients in each cluster and patients in other cluster(s) and healthy controls were compared. Comparision were carried out using the Wilcoxon test (significance set at $\mathrm{p}<0.005)$.

Finally, it was considered interesting to evaluate possible relationships between structural cluster characteristics and other biological and clinical dimensions. Thus, if a given set of structural variables was found to describe a distinct cluster of subjects, its relation to other variables significantly different in this cluster as compared to other patients was assessed using Pearson's rho coefficients.

\subsection{Results}

\subsubsection{Cluster identification}

The PCA analysis summary and the plots of the first two components for the four structural variables are shown in figure 3.1. Scatterplots in figure 3.1 clearly show two outliers, which were subsequently eliminated from remaining analyses. Also, from these graphs, the PC1CURV (PCA scores in the first factor for curvature) was selected as initial index for generating separate clusters. 

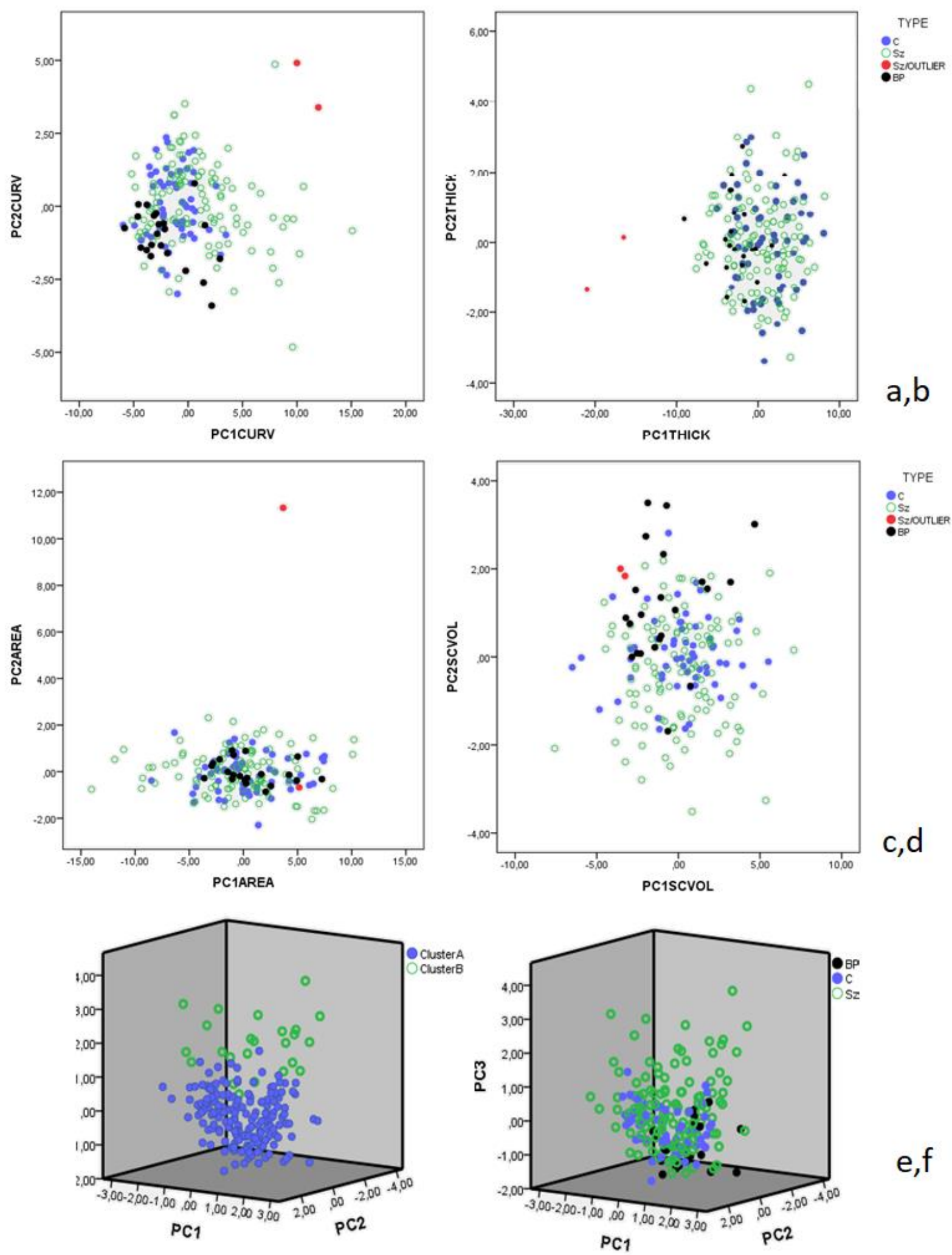

Figure 3.1. Scatter plots of the distribution of the values of the first two principal components for curvature, thickness, area and subcortical volume, respectively. Subjects are identified by color code. Outliers are marked in red. In the lower row, Scatter plots for the three PCs of the total set of MRI markers, showing different symbols for the final 
cluster and the type of observation. In the three dimensions scatter plot for these PCs, in figure 3.1e, the two clusters have been identified with different symbols, showing that patients identified primarily on the basis of higher mean curvature also showed globally smaller thickness and area. Moreover, in figure 3.1f, the observations have also been represented with different symbols for healthy controls, schizophrenia and bipolar disorder cases.

In the next step, PC1CURV was used to define three initial clusters: the first included 19 subjects with very large curvature values (G1: PC1CURV >4.5), the second included 30 subjects with high to moderate values (G2: 2.0 PC1CURV 4.5) and the third included 154 subjects with moderate to low values (G3: PC1CURV <2.0). In order to determine robust final clusters, an iterative process to allocate, one by one, individuals in G2 was designed. For each iteration, a LDF was derived using data from individuals in groups G1 and G3 and the whole data set. This function was then applied to individuals in G2, providing distance measurements to $\mathrm{G} 1$ and to $\mathrm{G} 3$. Then, the individual in $\mathrm{G} 2$ who was closest to G1 or G3, using these distances, was incorporated into the closest group for subsequent iterations. This process finished when all individuals in G2 were allocated into the other two groups (figure 3.2). The final LDF was a combination of standardized MRI data, as follows:

LDF=left superior frontal curvature*0.696+ left inferior parietal curvature*0.486right precentral thickness $* .468$-right caudal middle frontal area $* 0.269+$ right caudal middle frontal curvature*.346+right inferior parietal curvature*0.616-right caudal anterior cingulate curvature*0.324+left pallidum volume*0.238-left parahippocampal thickness*0.184-left caudal middle frontal area*0.223.

At the end of the process, two clusters were determined:

- $\quad$ Cluster A, including 97 Schizophrenia (52 FE) and all bipolar patients and healthy controls (figures 3.1 and 3.3).

- $\quad$ Cluster B, including 24 Schizophrenia, 12 of them FE patients. 
The LDF correctly classified, using cross-validation, $100 \%$ of the sample individuals in clusters A or B.

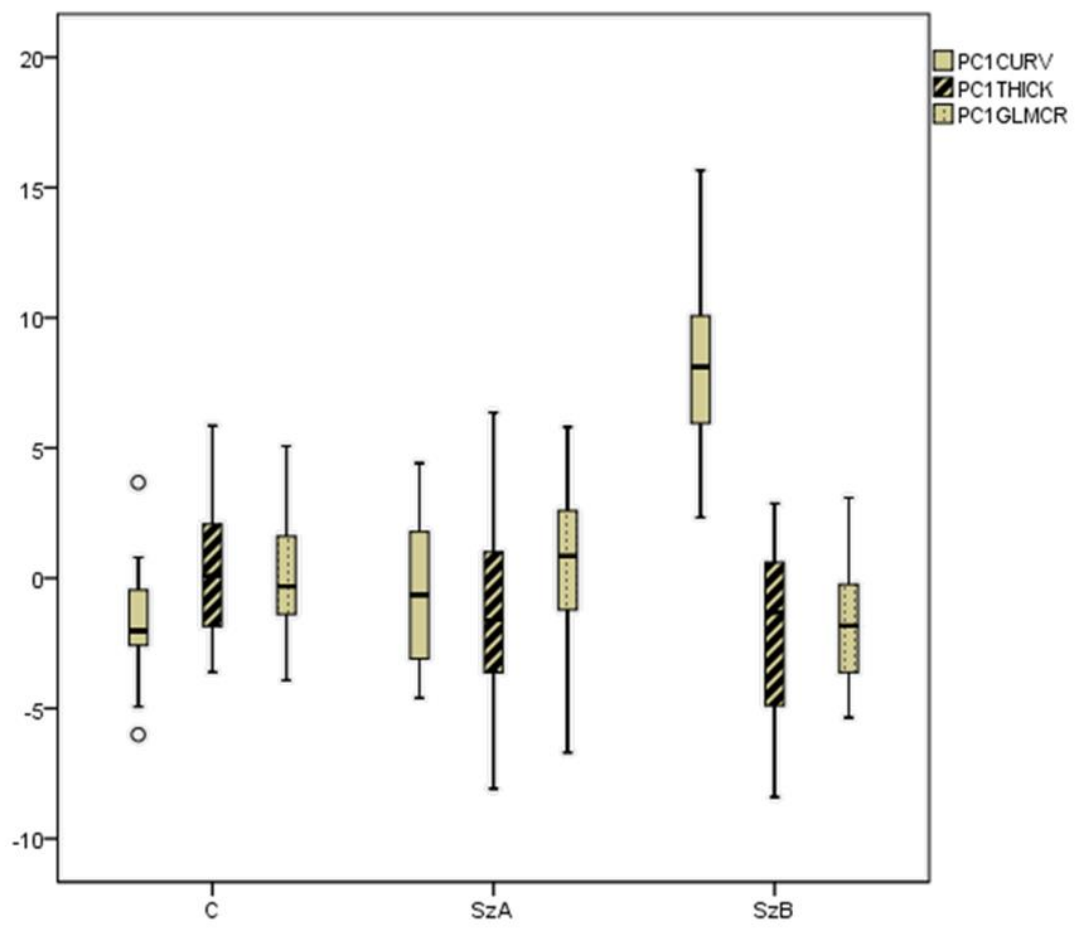

Figure 3.2. Distribution of factor scores for the PCA for curvature (PC1CURV), thickness (PC1THICK), and glucose metabolism (PC1GLMCR) in healthy controls (C), cluster $A$ schizophrenia patients (SzA) and cluster B schizophrenia patients (SzB).

There were no significant differences in sex distribution between subjects in clusters $A$ and $B\left(\chi^{2}=0.537, d f=1, p=0.463\right)$, age $(Z=0.470, p=0.639)$, previous typical or atypical treatment $\left(\chi^{2}=0.133 p, d f=1, p=0.989\right)$ or illness duration $(Z=-0.68, p=0.946)$ (Table 3.1). Cluster A included 136 subjects assessed with Philips scanner, 43 with 1.5 General Electrics, while cluster B included 16 assessed with Philips and 8 with 1.5 GE ( $\chi 2=0.84$, $\mathrm{df}=1, p=0.24)$.

However, there were weakly significant differences in slice thickness between cluster A (47: $1.5 \mathrm{~mm}$; 17: $2 \mathrm{~mm}$; 30: $2.20 \mathrm{~mm} ; 87: 3 \mathrm{~mm}$ ) and cluster $B(8: 1.5 \mathrm{~mm}$; 4: $2 \mathrm{~mm}$; $2.20 \mathrm{~mm} ; 3: 3 \mathrm{~mm})\left(\chi^{2}=8.54, \mathrm{df}=3, p=0.036\right)$. 
Table 3.1. Demographic, clinical, electrophysiological, and principal component values. Values are shown as means with standard deviation, SD, in brackets. Significance differences are shown with two types of symbols: ${ }^{*} p=0.08,{ }^{*} p=0.05,{ }^{*} p=0.01$ and ${ }^{* * *} p=0.001$ in comparison between schizophrenia clusters; ${ }^{\circ} p=0.05,{ }^{\circ 0} p=0.01$, ${ }^{\circ 00} p=0.001$ in comparison to healthy controls.

\begin{tabular}{|c|c|c|c|c|}
\hline & $\begin{array}{c}\text { Schizophrenia A } \\
\begin{array}{c}(n=97) \\
\text { Mean }\end{array}\end{array}$ & $\begin{array}{c}\text { Schizophrenia B } \\
\begin{array}{c}(n=24) \\
\text { Mean }\end{array}\end{array}$ & $\begin{array}{c}\text { Bipolar disorder } \\
\qquad \begin{array}{c}(n=22) \\
\text { Mean }\end{array}\end{array}$ & $\begin{array}{l}\text { Healthy controls } \\
\qquad \begin{array}{c}(n=60) \\
\text { Mean }\end{array}\end{array}$ \\
\hline Age (years) & $31.05(9.90)$ & $29.79(15.52)$ & $45.41(9.89)^{\circ \circ \circ}$ & $29.30(10.97)$ \\
\hline Sex distribution (F:M) & $37: 60$ & $11: 13$ & $10: 12$ & $27: 33$ \\
\hline FE/chronic & $52: 45$ & $12: 12$ & - & - \\
\hline Typical/atypical treatment (chronic) & $12: 33$ & 4:08 & - & - \\
\hline IIIness duration (months) & $54.87(74.34)$ & $55.87(106.87)$ & $143.46(84.96)$ & - \\
\hline Area factor scores & $0.03(3.60)$ & $-1.76(6.46)^{\circ}$ & $0.60(2.95)$ & $0.44(3.52)$ \\
\hline Curvature factor scores & $-0.53(2.44)$ & $7.20(3.19)^{* * * 000}$ & $-2.02(2.49)$ & $-1.28(1.92)$ \\
\hline Thickness factor scores & $0.40(3.75)$ & $-1.12(3.60)^{* \circ}$ & $-2.90(2.74)^{\circ}$ & $0.86(3.39)$ \\
\hline \multirow[t]{2}{*}{ Subcortical volumen factor scores } & $0.17(2.27)$ & $-0.21(2.96)$ & $-0.86(2.07)$ & $0.12(2.39)$ \\
\hline & $\begin{array}{c}\text { Schizophrenia A } \\
n=48\end{array}$ & $\begin{array}{c}\text { Schizophrenia B } \\
n=16\end{array}$ & - & $\begin{array}{c}\text { Healthy controls } \\
n=34\end{array}$ \\
\hline \multirow[t]{2}{*}{ Metabolism factor scores } & $0.54(2.76)$ & $-1.07(2.44)^{* * * \circ}$ & & $0.03(2.12)$ \\
\hline & $\begin{array}{c}\text { Schizophrenia A } \\
n=73\end{array}$ & $\begin{array}{c}\text { Schizophrenia B } \\
n=18\end{array}$ & - & - \\
\hline PANSS-positive & $23.69(6.48)$ & $24.82(6.90)$ & - & - \\
\hline PANSS-negative & $22.53(8.97)$ & $20.78(8.91)$ & - & - \\
\hline PANSS-general & $42.86(11.34)$ & $44.61(11.18)$ & - & - \\
\hline PANSS-p (\% improvement) & $40.59(25.30)$ & $44.15(28.76)$ & - & - \\
\hline PANSS-n (\% improvement) & $16.51(22.86)$ & $-9.26(61.59)^{*}$ & - & - \\
\hline \multirow[t]{2}{*}{ PANSS-g (\% improvement) } & $27.38(23.32)$ & $26.67(30.44)$ & - & - \\
\hline & $\begin{array}{c}\text { Schizophrenia A } \\
n=40\end{array}$ & $\begin{array}{c}\text { Schizophrenia B } \\
n=12\end{array}$ & - & $\begin{array}{c}\text { Healthy controls } \\
n=\mathbf{2 4}\end{array}$ \\
\hline P300 amplitude in $\mu \mathrm{V}$ & $6.17(4.61)^{000}$ & $8.42(5.02)^{\circ}$ & - & $11.72(2.72)$ \\
\hline P300 latency in ms & $310.40(100.95)$ & 302.00 (106.99) & - & $324.00(30.55)$ \\
\hline
\end{tabular}

Thus, to test if slice thickness played a major role in cluster definition two issues were addressed: i) It was verified that after withdrawing cases with $3 \mathrm{~mm}$ slice thickness, PCA yields quite similar outputs to those obtained using the whole sample. In particular, both clusters are clearly separated in the new 3-dimensional PCA scatterplot (figure S3.1) and ii) It was shown that there were not significant differences between means across the 
slice thickness groups using the LDF, an index that completely separates the clusters, (figure S3.2). Therefore, slice thickness can be discarded as a factor in cluster formation

A final PCA from area, thickness and curvature was conducted to represent the most relevant information (figure 3.1). The first three principal components accounted for $22 \%, 16 \%$, and $14 \%$ of the variance, respectively. From this graphical representation, the same cluster of cases may be identified, underlining the robustness of the results.

\subsubsection{Cluster validation}

3.3.2.1. Comparison of MRI parameters between patient clusters and healthy controls

\subsection{Curvature}

Mean curvature was larger in Cluster B schizophrenia patients compared to healthy controls in most regions included in analysis (table 3.2): right caudal anterior cingulate $(Z=2.97, p=0.003)$, bilateral caudal middle frontal (left $Z=5.97, p<0.0001$; right $Z=6.32$, $p<0.0001$ ), cuneus (left $Z=5.50, p<0.0001$; right $Z=5.51, p<0.0001$ ), inferior parietal (left $Z=6.84, p<0.0001$; right $Z=6.77, p<0.0001$ ), medial orbitofrontal (left $Z=4.92, p<0.0001$; right $Z=5.09, p<0.0001$ ), parahippocampal (left $Z=4.98, p<0.0001$; right $Z=3.43$, $p=0.0006$ ), pars orbitalis (left $Z=5.81, p<0.0001$; right $Z=4.99, p<0.0001$ ), pars triangularis (left $Z=5.89, p<0.0001$; right $Z=6.49, p<0.0001$ ), precentral (left $Z=6.13, p<0.0001$; right $Z=6.31, p<0.0001$ ), rostral anterior cingulate (left $Z=3.66, p=0.0002$; right $Z=3.66$, $p=0.0002$ ), rostral middle frontal (left $Z=6.64, p<0.0001$; right $Z=6.80, p<0.0001$ ), superior frontal (left $Z=6.98, p<0.0001$; right $Z=6.90, p<0.0001$ ), superior temporal (left $Z=6.25, p<0.0001$; right $Z=6.07, p<0.0001$ ) and insula (left $Z=3.22, p=0.001$; right $Z=3.91$, $p<0.0001)$.

Cluster A schizophrenia patients only showed larger curvature values in comparison to healthy controls in right rostral anterior cingulate $(Z=-2.92, p=0.003)$ (table 3.2, figure $3.2)$.

\subsection{Thickness}

Cluster B schizophrenia patients showed a modest thinning in comparison to healthy controls at left caudal anterior cingulate $(Z=-3.46, p=0.0005$, right superior frontal $(Z=-$ 
3.28, $p=0.001)$, precentral $(Z=-3.05 p=0.002)$ and right caudal middle frontal $(Z=-2.83$, $p=0.0046)$.

Cluster A schizophrenia patients showed no thickness differences in comparison to healthy controls.

\subsection{Area}

In comparison to healthy controls, neither cluster B nor cluster A schizophrenia patients showed a significant difference in area values.

\subsection{Subcortical volumes}

Neither type A nor type B patients showed significant subcortical volume differences in comparison to healthy controls (Supplementary table 3.1).

\subsubsection{Comparison of other parameters between clusters of schizophrenia} patients

\subsection{Resting metabolism}

Cluster B patients had significantly smaller glucose metabolism than cluster A schizophrenia patients in left thalamus $(Z=-3.03 . p=0.002)$ and left caudate $(Z=-2.87$. $p=0.004$ ) (figure 3.2. table 3.3).

\subsection{P300 parameters}

There was no significant effect of cluster on P300 parameters (table 3.1).

\subsection{Clinical data}

Baseline scores did not differ between patient clusters (table 3.1). However, cluster A schizophrenia patients showed a modest improvement in negative symptoms, while cluster B schizophrenia patients exhibited a modest worsening at follow-up $(Z=-1.74$. $p=0.08)$. 
Table 3.2. Cortical curvature, thickness and area values for every cluster. The data are shown as means and standard deviation in brackets. Significance differences are shown with two types of symbols, each one with three categories: ${ }^{*} p=0.005$, ${ }^{* *} p=0.001$ and ${ }^{* * *} p=0.0001$ in comparison between schizophrenia clusters; ${ }^{\circ} p=0.005,{ }^{\circ 0} p=0.001$, and ${ }^{\circ 0 \circ} p=0.0001$ in comparison to healthy controls

\begin{tabular}{|c|c|c|c|c|c|c|c|c|c|c|c|c|c|c|c|c|}
\hline & \multicolumn{4}{|c|}{ Schizophrenia A ( $n=97)$} & \multicolumn{4}{|c|}{ Schizophrenia B ( $n=24)$} & \multicolumn{4}{|c|}{ Bipolar disorder ( $n=22)$} & \multicolumn{4}{|c|}{ Controls $(n=60)$} \\
\hline & \multicolumn{2}{|c|}{ Left } & \multicolumn{2}{|c|}{ Right } & \multicolumn{2}{|c|}{ Left } & \multicolumn{2}{|c|}{ Right } & \multicolumn{2}{|c|}{ Left } & \multicolumn{2}{|c|}{ Right } & \multicolumn{2}{|c|}{ Left } & \multicolumn{2}{|c|}{ Right } \\
\hline & Mean & SD & Mean & SD & Mean & SD & Mean & SD & Mean & SD & Mean & SD & Mean & SD & Mean & SD \\
\hline \multicolumn{17}{|l|}{ Cortical curvature $(1 / \mathrm{mm})$} \\
\hline Caudal anterior cingulate & 0.15 & 0.02 & 0.16 & 0.01 & 0.16 & 0.02 & $0.17^{\circ}$ & 0.02 & 0.15 & 0.02 & 0.15 & 0.01 & 0.15 & 0.01 & 0.15 & 0.01 \\
\hline Caudal middle frontal & 0.15 & 0.01 & 0.15 & 0.01 & $0.17 * * * 000$ & 0.02 & $0.18 * * * 000$ & 0.01 & 0.15 & 0.01 & 0.14 & 0.01 & 0.15 & 0.01 & 0.15 & 0.01 \\
\hline Cuneus & 0.17 & 0.02 & 0.17 & 0.01 & $0.19 * * * 000$ & 0.02 & $0.19 * * * 000$ & 0.03 & 0.16 & 0.01 & 0.16 & 0.01 & 0.17 & 0.01 & 0.17 & 0.01 \\
\hline Inferior parietal & 0.15 & 0.01 & 0.15 & 0.01 & $0.17 * * * \circ 00$ & 0.01 & $0.17 * * * 000$ & 0.01 & 0.14 & 0.01 & 0.14 & 0.01 & 0.15 & 0.01 & 0.15 & 0.01 \\
\hline Medial orbitofrontal & 0.17 & 0.01 & 0.17 & 0.02 & $0.18 * * * 000$ & 0.02 & $0.18 * * 000$ & 0.02 & 0.16 & 0.01 & 0.15 & 0.01 & 0.16 & 0.01 & 0.16 & 0.01 \\
\hline Para hippocampal & 0.14 & 0.02 & 0.14 & 0.02 & $0.17 * * * 000$ & 0.03 & $0.16 * * \circ$ & 0.03 & 0.14 & 0.02 & 0.14 & 0.02 & 0.14 & 0.02 & 0.14 & 0.02 \\
\hline Pars orbitalis & 0.18 & 0.02 & 0.18 & 0.02 & $0.21 * * * \circ 00$ & 0.02 & $0.22 * * * 000$ & 0.04 & 0.19 & 0.03 & 0.18 & 0.03 & 0.18 & 0.02 & 0.18 & 0.01 \\
\hline Pars triangularis & 0.15 & 0.01 & 0.15 & 0.01 & $0.18 * * * 000$ & 0.02 & $0.18 * * * 000$ & 0.02 & 0.15 & 0.02 & 0.15 & 0.01 & 0.15 & 0.01 & 0.15 & 0.01 \\
\hline Pre central & 0.15 & 0.01 & 0.15 & 0.01 & $0.18 * * * 000$ & 0.02 & $0.18 * * * 000$ & 0.02 & 0.15 & 0.01 & 0.15 & 0.01 & 0.15 & 0.01 & 0.15 & 0.01 \\
\hline Rostral anterior cingulate & 0.15 & 0.01 & 0.15 & $0.02^{\circ}$ & $0.17 * * \circ$ & 0.02 & $0.16^{\circ 0}$ & 0.02 & 0.15 & 0.01 & 0.15 & 0.02 & 0.15 & 0.01 & 0.15 & 0.01 \\
\hline Rostral middle frontal & 0.17 & 0.01 & 0.17 & 0.01 & $0.19 * * * 000$ & 0.01 & $0.19 * * * \circ 0 \circ$ & 0.02 & 0.16 & 0.01 & 0.17 & 0.01 & 0.16 & 0.01 & 0.17 & 0.01 \\
\hline Superior frontal & 0.15 & 0.01 & 0.16 & 0.01 & $0.18 * * * 000$ & 0.01 & $0.18 * * * 000$ & 0.01 & 0.15 & 0.01 & 0.15 & 0.01 & 0.15 & 0.01 & 0.15 & 0.01 \\
\hline Superior temporal & 0.14 & 0.01 & 0.15 & 0.02 & $0.17 * * * 000$ & 0.01 & $0.17 * * * \circ 00$ & 0.02 & 0.14 & 0.02 & 0.14 & 0.01 & 0.14 & 0.02 & 0.14 & 0.01 \\
\hline Insula & 0.14 & 0.02 & 0.16 & 0.02 & $0.16^{* * \circ}$ & 0.02 & $0.18 * * * 000$ & 0.02 & 0.15 & 0.03 & 0.16 & 0.01 & 0.15 & 0.02 & 0.16 & 0.02 \\
\hline \multicolumn{17}{|l|}{ Cortical thickness ( $\mathrm{mm}$ ) } \\
\hline Caudal anterior cingulate & 2.77 & 0.32 & 2.78 & 0.30 & $2.57^{\circ 0}$ & 0.28 & 2.65 & 0.28 & 2.72 & 0.40 & 2.69 & 0.34 & 2.87 & 0.35 & 2.79 & 0.29 \\
\hline Caudal middle frontal & 2.42 & 0.21 & 2.41 & 0.19 & 2.37 & 0.22 & $2.30^{\circ}$ & 0.19 & 2.25 & 0.20 & 2.26 & 0.16 & 2.44 & 0.21 & 2.45 & 0.20 \\
\hline Cuneus & 1.79 & 0.15 & 1.82 & 0.18 & 1.82 & 0.30 & 1.88 & 0.37 & 1.73 & 0.17 & 1.74 & 0.16 & 1.76 & 0.17 & 1.78 & 0.19 \\
\hline Inferior parietal & 2.30 & 0.15 & 2.36 & 0.15 & 2.24 & 0.20 & 2.29 & 0.21 & 2.24 & 0.13 & 2.28 & 0.17 & 2.30 & 0.17 & 2.36 & 0.17 \\
\hline Medial orbitofrontal & 2.52 & 0.24 & 2.50 & 0.22 & 2.52 & 0.26 & 2.52 & 0.33 & 2.42 & 0.18 & 2.46 & 0.24 & 2.56 & 0.23 & 2.52 & 0.25 \\
\hline
\end{tabular}




\begin{tabular}{|c|c|c|c|c|c|c|c|c|c|c|c|c|c|c|c|c|}
\hline Para hippocampal & 2.49 & 0.39 & 2.45 & 0.31 & $2.22 * *$ & 0.27 & 2.27 & 0.25 & 2.27 & 0.29 & 2.29 & 0.21 & 2.42 & 0.37 & 2.38 & 0.29 \\
\hline Pars orbitalis & 2.53 & 0.32 & 2.49 & 0.30 & 2.48 & 0.38 & 2.47 & 0.40 & 2.18 & 0.25 & 2.28 & 0.27 & 2.52 & 0.29 & 2.48 & 0.26 \\
\hline Pars triangularis & 2.39 & 0.21 & 2.38 & 0.18 & 2.37 & 0.26 & 2.35 & 0.27 & 2.26 & 0.23 & 2.24 & 0.17 & 2.40 & 0.22 & 2.41 & 0.19 \\
\hline Pre central & 2.36 & 0.21 & 2.34 & 0.21 & 2.26 & 0.21 & $2.22^{\circ}$ & 0.21 & 2.12 & 0.17 & 2.12 & 0.15 & 2.38 & 0.19 & 2.38 & 0.19 \\
\hline Rostral anterior cingulate & 2.92 & 0.27 & 2.92 & 0.31 & 2.79 & 0.33 & 2.81 & 0.27 & 2.70 & 0.25 & 2.73 & 0.27 & 2.98 & 0.29 & 2.98 & 0.28 \\
\hline Rostral middle frontal & 2.27 & 0.21 & 2.27 & 0.19 & 2.30 & 0.25 & 2.30 & 0.24 & 2.10 & 0.15 & 2.13 & 0.16 & 2.30 & 0.20 & 2.30 & 0.19 \\
\hline Superior frontal & 2.66 & 0.22 & 2.64 & 0.19 & 2.57 & 0.20 & $2.55^{\circ}$ & 0.19 & 2.47 & 0.17 & 2.48 & 0.19 & 2.71 & 0.21 & 2.72 & 0.17 \\
\hline Superior temporal & 2.51 & 0.23 & 2.51 & 0.24 & 2.38 & 0.23 & 2.40 & 0.23 & 2.42 & 0.14 & 2.38 & 0.16 & 2.53 & 0.21 & 2.54 & 0.21 \\
\hline Insula & 2.99 & 0.20 & 2.94 & 0.22 & 2.89 & 0.22 & 2.84 & 0.23 & 2.91 & 0.15 & 2.79 & 0.21 & 2.98 & 0.23 & 2.96 & 0.21 \\
\hline \multicolumn{17}{|l|}{ Cortical área $\left(\mathrm{mm}^{2}\right)$} \\
\hline Caudal anterior cingulate & 632.62 & 139.79 & 729.54 & 142.59 & 571.29 & 157.54 & 687.79 & 244.84 & 628.77 & 128.40 & 748.68 & 150.36 & 626.67 & 114.51 & 739.75 & 145.81 \\
\hline Caudal middle frontal & 2.381 .29 & 359.56 & 2.167 .52 & 398.71 & 2.153 .75 & 577.70 & 2.026 .25 & 533.28 & 2.477 .14 & 419.05 & 2.344 .91 & 432.70 & 2.396 .33 & 453.16 & 2.169 .42 & 387.07 \\
\hline Cuneus & 1.387 .10 & 219.80 & 1.472 .12 & 231.63 & 1.265 .58 & 311.53 & 1.334 .46 & 319.46 & 1.374 .59 & 195.19 & 1.404 .32 & 157.84 & 1.379 .72 & 175.41 & 1.486 .77 & 208.20 \\
\hline Inferior parietal & 4.440 .72 & 628.73 & 5.209 .14 & 751.45 & 4.433 .08 & 1.123 .59 & 5.029 .42 & 1.308 .08 & 4.477 .73 & 604.67 & 5.509 .41 & 767.63 & 4.640 .20 & 731.46 & 5.355 .18 & 733.19 \\
\hline Medial orbitofrontal & 1.816 .48 & 281.29 & 1.743 .64 & 255.91 & 1.660 .25 & 418.44 & 1.588 .88 & 424.38 & 1.866 .64 & 279.29 & 1.681 .00 & 219.34 & 1.866 .13 & 279.40 & 1.745 .07 & 260.98 \\
\hline Para hippocampal & 683.45 & 89.71 & 662.40 & 102.73 & 740.58 & 269.44 & 644.04 & 163.61 & 693.45 & 88.07 & 680.18 & 64.93 & 705.28 & 145.30 & 693.97 & 105.60 \\
\hline Pars orbitalis & 637.27 & 100.65 & 784.49 & 126.28 & 583.21 & 138.21 & 733.67 & 183.66 & 667.50 & 128.40 & 796.09 & 145.89 & 654.53 & 77.70 & 797.40 & 108.49 \\
\hline Pars triangularis & 1.269 .51 & 210.75 & 1.502 .27 & 274.46 & 1.180 .21 & 236.31 & 1.359 .33 & 331.85 & 1.296 .41 & 255.93 & 1.485 .77 & 272.32 & 1.292 .13 & 219.88 & 1.441 .08 & 229.32 \\
\hline Pre central & 5.028 .60 & 697.27 & 4.988 .73 & 640.53 & 4.917 .42 & 1.018 .04 & 4.864 .04 & 971.81 & 5.268 .48 & 526.22 & 5.332 .14 & 608.46 & 5.068 .40 & 616.95 & 5.093 .12 & 621.59 \\
\hline Rostral anterior cingulate & 773.69 & 151.86 & 630.25 & 133.77 & 677.50 & 224.54 & 560.88 & 210.02 & 804.48 & 166.56 & 617.23 & 130.69 & 791.17 & 148.86 & 601.90 & 140.65 \\
\hline Rostral middle frontal & 5.743 .54 & 968.99 & 5.896 .24 & 925.88 & 5.122 .29 & 1.635 .49 & 5.299 .54 & 1.628 .71 & 5.933 .90 & 763.35 & 6.204 .09 & 1.005 .00 & 5.888 .22 & 843.59 & 5.977 .92 & 840.58 \\
\hline Superior frontal & 7.256 .28 & 983.44 & 6.952 .41 & 959.53 & 7.045 .33 & 1.628 .92 & 6.585 .71 & 1.475 .44 & 7.425 .14 & 823.80 & 7.067 .59 & 930.96 & 7.313 .22 & 988.94 & 6.991 .13 & 874.27 \\
\hline Superior temporal & 3.701 .63 & 488.21 & 3.439 .22 & 431.27 & 3.572 .38 & 821.71 & 3.311 .13 & 715.76 & 3.793 .19 & 460.08 & 3.687 .41 & 510.54 & 3.753 .40 & 549.13 & 3.510 .15 & 470.87 \\
\hline Insula & 2.103 .54 & 280.38 & 2.204 .28 & 311.98 & 2.134 .92 & 382.14 & 2.171 .50 & 390.55 & 2.192 .67 & 232.70 & 2.371 .50 & 347.38 & 2.140 .32 & 244.24 & 2.231 .53 & 322.35 \\
\hline
\end{tabular}


Table 3.3. Metabolic activity values. The data are shown as means and standard deviation in brackets. Significance differences are shown with two types of symbols, each one with three categories: $* p<0.005, * * p<0.001$ and $* * * p<0.0001$ in comparison between schizophrenia clusters; ${ }^{\circ} p<0.005,{ }^{\circ 0} p<0.001,{ }^{\circ 00} p<0.0001$ in comparison to healthy controls

\begin{tabular}{|c|c|c|c|c|c|c|c|c|c|c|c|c|}
\hline \multirow[t]{3}{*}{ Metabolic activity } & \multicolumn{4}{|c|}{ Schizophrenia A ( $n=48)$} & \multicolumn{4}{|c|}{ Schizophrenia B $(n=16)$} & \multicolumn{4}{|c|}{ Controls ( $n=34)$} \\
\hline & \multirow{2}{*}{$\begin{array}{c}\text { Left } \\
\text { Mean }\end{array}$} & \multicolumn{3}{|c|}{ Right } & \multirow{2}{*}{$\begin{array}{c}\text { Left } \\
\text { Mean }\end{array}$} & \multicolumn{3}{|c|}{ Right } & \multirow{2}{*}{$\begin{array}{c}\text { Left } \\
\text { Mean }\end{array}$} & \multicolumn{3}{|c|}{ Right } \\
\hline & & SD & Mean & SD & & SD & Mean & SD & & SD & Mean & SD \\
\hline Cingulus & 101.68 & 6.05 & 101.50 & 6.22 & $97.49^{\circ}$ & 3.92 & 98.37 & 4.93 & 101.98 & 5.06 & 101.51 & 4.84 \\
\hline Superior temporal gyrus & 96.75 & 4.81 & 98.26 & 3.51 & 97.06 & 4.37 & 98.51 & 4.09 & 98.36 & 3.53 & 97.66 & 4.04 \\
\hline Frontal grey matter & 104.28 & 3.28 & 106.98 & 3.41 & 104.33 & 5.37 & 106.41 & 4.03 & 106.71 & 3.48 & 107.77 & 3.42 \\
\hline Occipital grey matter & 104.10 & 3.91 & 103.39 & 4.33 & 107.31 & 4.39 & 106.75 & 4.99 & 105.62 & 3.74 & 104.76 & 3.52 \\
\hline Orbital grey matter & 96.61 & 4.50 & 96.43 & 4.45 & 94.06 & 4.80 & 93.44 & 5.38 & 97.47 & 4.23 & 96.52 & 4.14 \\
\hline Parietal grey matter & 103.59 & 2.71 & $104.56^{\circ}$ & 3.39 & 103.65 & 3.45 & $105.42^{\circ}$ & 4.53 & 102.15 & 2.80 & 102.01 & 2.64 \\
\hline Total temporal GM & 91.61 & 2.81 & 92.16 & 2.29 & 91.62 & 3.47 & 91.74 & 3.25 & 91.59 & 3.13 & 91.42 & 3.13 \\
\hline Pallidum putamen & $117.78^{\circ \circ}$ & 9.49 & $118.39^{\circ 00}$ & 9.15 & 112.78 & 9.45 & 114.61 & 10.98 & 109.68 & 8.79 & 108.97 & 8.69 \\
\hline Thalamus & 102.25 & 8.56 & 103.04 & 9.05 & $95.16^{* \circ}$ & 6.50 & 97.00 & 6.41 & 101.63 & 7.57 & 101.36 & 7.13 \\
\hline Caudate & 108.95 & 11.82 & 109.91 & 11.12 & 99.69* & 11.43 & 102.79 & 10.79 & 104.61 & 8.56 & 105.16 & 8.17 \\
\hline
\end{tabular}

\subsubsection{Comparison of other parameters between clusters of schizophrenia} patients and healthy controls.

\subsection{Glucose metabolism}

Cluster B patients showed significantly reduced left cingulate $(Z=-2.98 . p=0.003)$ and left thalamic $(\mathrm{Z}=-2.85, p=0.004)$ activity in comparison to healthy controls.

Cluster A schizophrenia patients showed a significantly larger putaminal (Left Z=3.78, $p=0.0001$; Right $Z=4.14, p<0.0001$ ) metabolism than healthy controls (figure 3.3 ).

Both A and B clusters showed a significantly larger metabolic activity in right parietal grey matter (Cluster A $Z=-3.05, p=0.002$; Cluster $B Z=2.98, p=0.003$ ) in comparison to healthy controls (table 3.3).

\subsection{P300 parameters}

Patients in the two clusters showed significant decrease in P300 amplitude, but not in latency (Cluster A: Z=4.98, $p<0.001$; Cluster B: $Z=-2.39, p=0.016$ ) (table 3.1). 


\subsubsection{Association between curvature, metabolism and clinical variables}

There was a significant association between PC1CURV (explaining most of curvature variance) and PRINM1 (explaining most of metabolism variance). The relation was negative (rho $=-0.326 . p=0.001$ ). Thus, larger curvature values were associated with lower metabolic activity in the regions included in the study.

There was a weak association between PC1CURV and improvement on negative symptoms (more curvature, less improvement; rho=-0.245, $n=64, p=0.05$ ). Basal symptoms were unrelated to curvature.

\subsection{Discussion}

A function, mostly contributed by cortical curvature, segregated $20 \%$ of subjects within the schizophrenia group (cluster B), who also showed a more modest cortical thinning and hypometabolism in the left cingulate and thalamus, together with persistent negative symptoms. Cluster A schizophrenia patients showed normal structure but higher putaminal glucose metabolism than healthy controls. Metabolism and clinical improvement were negatively related to curvature. All bipolar and healthy controls subjects were grouped within schizophrenia cluster A. Therefore, clear, qualitative differences in potentially relevant biological parameters were observed between syndromically-similar patients.

This study's findings are in partial agreement with Crow's theory of two syndromes in schizophrenia ${ }^{247}$. Type B in the current study overlaps partly with Crow's type II (poor treatment response, structural abnormalities). However, Type A and B patients within this study did not differ in basal symptoms and seemed to correspond to different patients. In Crow's theory, structural abnormalities centred around ventricular volumes 247, however, our study used MRI parameters less dependent on cranial size. This approach has the advantage of being data-driven, where all cases were introduced a priori into the clustering analyses, without taking into consideration patients' diagnoses.

A number of schizophrenia patients (schizophrenia cluster A) showed significantly increased curvature, which correlates with reports of high ${ }^{233}$, normal ${ }^{234}$ and reduced 235 gyrification within this syndrome. The relevance of this structural alteration is 
underlined by reduced subcortical metabolism within this group and its equal proportion within FE and chronic patients. An alteration in cortical gyrification in schizophrenia may reflect neurodevelopmentally-determined disturbed connectivity 248. In particular, short-range connectivity secondary to under-developed expansion of upper cortical layers ${ }^{249}$. This may warrant the interest of assessing cortical folding in relation to measures of functional connectivity found to be altered in schizophrenia ${ }^{96}$. Increased curvature has been related to white matter pathology ${ }^{250}$, and myelin alteration may be found in some schizophrenia patients ${ }^{251}$, which, according to this study's data, may be speculatively more characteristic of cluster B schizophrenia patients.

Furthermore, only cluster A showed a significant increase of putaminal activity. Since antipsychotics induce putaminal metabolic increase ${ }^{252}$, and their absence is reportedly related to bad prognosis in schizophrenia ${ }^{253}$, as well as to resistance to clozapine ${ }^{254}$, the lack of this expected putaminal hyperactivation may characterize a biologically-discrete schizophrenia subtype along with persistent negative symptoms and increased mean curvature, according to this study's results.

Other metabolic findings in cluster B were reduced metabolism in the left anterior cingulate and thalamus. Metabolic activity was negatively associated with curvature, suggesting a common underpinning for structural and metabolic abnormalities. Cingulate hypometabolism has also been reported in chronic ${ }^{255}$ and unmedicated 256 schizophrenia. Thalamic hypoactivity distinguishes between patients that have or have not responded to clozapine ${ }^{254}$.

Contrary to our hypothesis, cortical thickness was less efficient in cluster definition than curvature. Since most of the treatment-resistant patients were discarded because of poorer MR quality, it cannot be discarded that such cases may show larger thinning. Moreover, the classificatory power of cortical thickness might be blurred by antipsychotics, since these drugs may reduce grey matter volumes ${ }^{257}$.

Most schizophrenia patients, as well as all the bipolar ones, clustered together with healthy controls. This may have consequences upon the interpretation of research results in the field, since the joint consideration of (at least) clusters A and B may obscure their respective particular characteristics. This may lead to the erroneous conclusion 
that abnormalities such as abnormal folding or thalamic and cingulate hypoactivity are uncharacteristic of schizophrenia, since they might only be found in a subtype within that syndrome. A recent report has shown disjoint associations between networks of SNPs sets and clinical phenotypes ${ }^{58}$. Two of these SNPs clusters were associated with severe process (respectively with positive or with negative and positive symptoms), hence supporting the possibility of uncovering the genetic background of at least some of the subgroups here identified.

The present study has several limitations. Sample size is low and perhaps larger samples may reveal further valid clusters in schizophrenia and bipolar patients. Metabolic and electrophysiological data were available only for a subset of cases, as well as clinical outcomes. Moreover, it would be of great interest to have collected cognitive assessments measurements of daily functioning and a larger sample, especially of nevertreated patients, as well as other biological parameters, such as neuronal and glial markers. Slice thickness differed between 1.5 and $3 \mathrm{~mm}$, but its influence on cluster definition seemed negligible and this may facilitate the clinical usefulness of the found subtypes, since it may not depend on particular acquisition parameters.

As a conclusion, the variability here described (patients with and without cortical thinning and/or mean curvature associated with other features) may contribute to explain the still insufficient replication of imaging findings in schizophrenia. We propose the segregation within the schizophrenia syndrome of patients with large cortical curvature, persistent negative symptoms, thalamic and cingulate hypoactivity and a lack of putaminal metabolic increase with antipsychotics, which may constitute a focus for genetic research. Biologically-based subtypes may, therefore, offer a new framework for the identification of substrates of the illnesses included in the schizophrenia syndrome. Although at the current stage the possibility that our findings may have clinical utility remains preliminary, similar classifications may be useful in the near future to identify novel therapeutic targets in some of the schizophrenia clusters. 


\subsection{Supplementary materials}

Supplementary table 3.1. Subcortical volumes values. The data are shown as mean and standard deviation in brackets. There were no significant differences in any comparison

\begin{tabular}{|c|c|c|c|c|c|c|c|c|c|c|c|c|c|c|c|}
\hline \multirow{3}{*}{$\begin{array}{c}\text { Subcortical } \\
\text { volume } \\
\text { (mm3) }\end{array}$} & \multicolumn{4}{|c|}{ Schizophrenia A ( $n=97)$} & \multicolumn{4}{|c|}{ Schizophrenia B $(n=24)$} & \multicolumn{4}{|c|}{ Bipolar disorder $(n=22)$} & \multicolumn{3}{|c|}{ Controls $(n=60)$} \\
\hline & \multicolumn{2}{|c|}{ Left } & \multicolumn{2}{|c|}{ Right } & \multicolumn{2}{|c|}{ Left } & \multicolumn{2}{|c|}{ Right } & \multicolumn{2}{|l|}{ Left } & \multicolumn{2}{|c|}{ Right } & \multicolumn{2}{|c|}{ Left } & Right \\
\hline & Mean & SD & Mean & SD & Mean & SD & Mean & SD & Mean & SD & Mean & SD & Mean & SD & Mean SD \\
\hline Thalamus & 7961,1 & 1052,6 & 7065,4 & 851,27 & 7752,8 & 1304,8 & 6914,5 & 1103,5 & 8492,1 & 1175,9 & 7110,3 & 730,8 & 8064,6 & 1097,1 & $7196,1 \quad 849,75$ \\
\hline Caudate & 3573,3 & 459,73 & 3699,4 & 500,99 & 3592,5 & 656,1 & 3718,5 & 709,27 & 3159,3 & 466,63 & 3302 & 544,27 & 3525,7 & 562,13 & $3740,1 \quad 586,56$ \\
\hline Putamen & 5518,9 & 843,82 & 5371,1 & 782,76 & 5413,5 & 919,98 & 5244,9 & 931,57 & 4919,4 & 813,02 & 4879,3 & 698,41 & 5349,6 & 801,08 & $5222,7 \quad 766,71$ \\
\hline Pallidum & 1625,4 & 349,07 & 1700,5 & 316,32 & 1648,6 & 466,72 & 1615,1 & 382,65 & 1405,8 & 267,68 & 1505,1 & 241,84 & 1612,0 & 305,75 & $1672,3 \quad 305,31$ \\
\hline \multirow[t]{2}{*}{ Hippocampus } & 4357,2 & 446,97 & 4216,2 & 444,46 & 4205,2 & 562,81 & 4152,6 & 520,95 & 4476,3 & 508,46 & 4350,1 & 422,96 & 4377,3 & 482,22 & $4277,4 \quad 468,58$ \\
\hline & \multicolumn{2}{|c|}{ Mean } & \multicolumn{2}{|c|}{ SD } & \multicolumn{2}{|c|}{ Mean } & \multicolumn{2}{|c|}{ SD } & \multicolumn{2}{|c|}{ Mean } & \multicolumn{2}{|c|}{ SD } & \multicolumn{2}{|c|}{ Mean } & SD \\
\hline Total intra cranial & \multicolumn{2}{|c|}{$1,464,170.66$} & \multicolumn{2}{|c|}{$163,422.41$} & \multicolumn{2}{|c|}{$1,425,946.68$} & \multicolumn{2}{|c|}{$208,747.12$} & \multicolumn{2}{|c|}{$1,473,039.08$} & \multicolumn{2}{|c|}{$171,538.27$} & \multicolumn{2}{|c|}{$1,477,008.32$} & $157,357.41$ \\
\hline
\end{tabular}




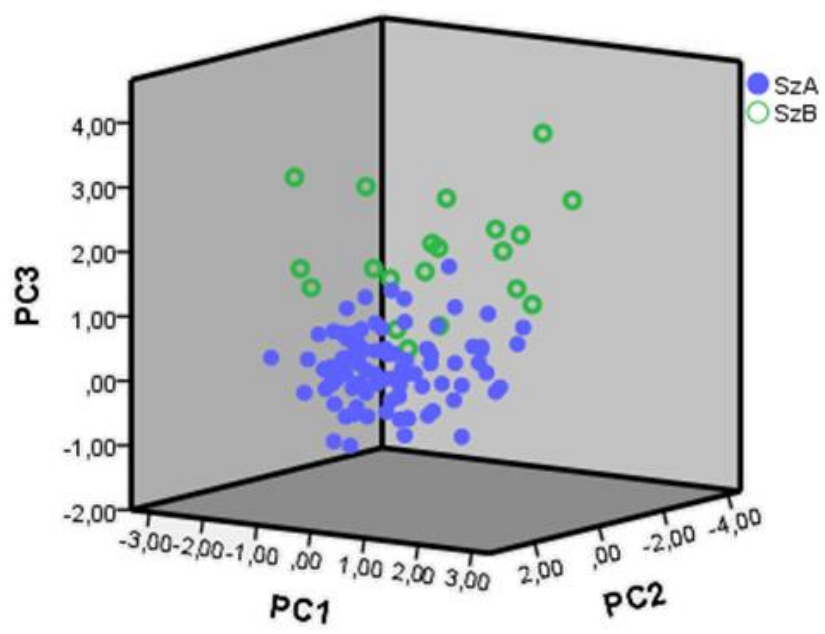

Supplementary figure 3.1. Scatterplot for the three PCS of the total set of MRI markers excluding those magnetic resonance whose slice thickness were $3 \mathrm{~mm}$.

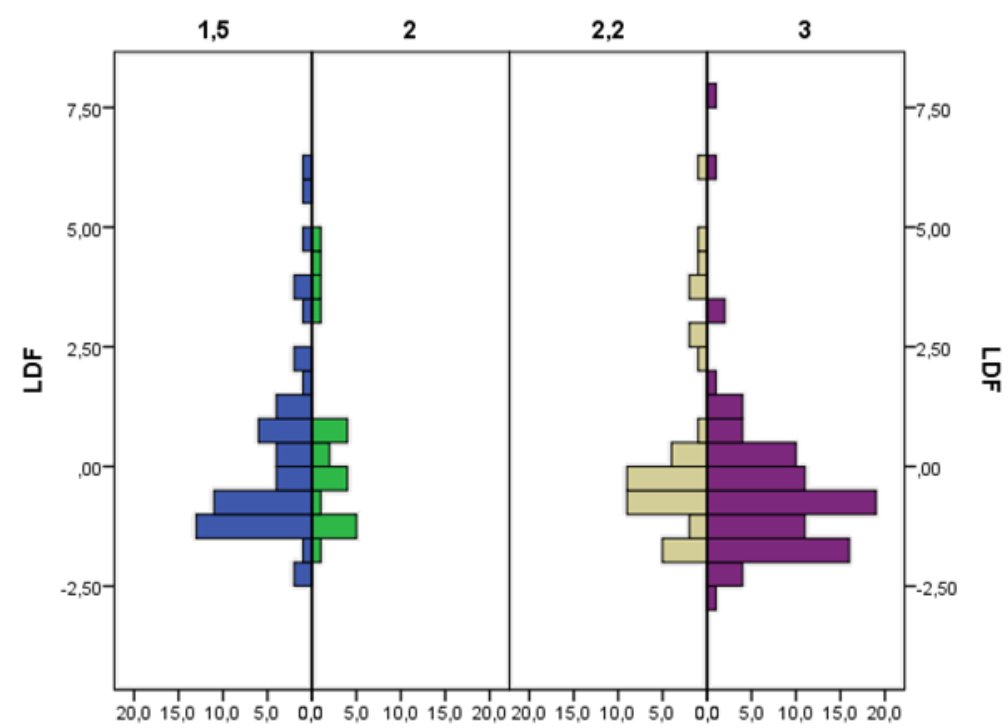

\begin{tabular}{l|c|c|c}
\hline $\begin{array}{c}\text { Slice thickness } \\
\text { comparison }\end{array}$ & $\begin{array}{c}\text { Mean } \\
\text { differences }\end{array}$ & \multicolumn{2}{|c}{$\begin{array}{c}\text { Confidence } \\
\text { interval }\end{array}$} \\
\hline $\mathbf{2 - 2 . 2}$ & 0.08 & -11.92 & 13.67 \\
$\mathbf{2 - 1 . 5}$ & 0.19 & -10.11 & 14.10 \\
$\mathbf{2 - 3}$ & 0.88 & -0.26 & 20.29 \\
$\mathbf{2 . 2}-\mathbf{1 . 5}$ & 0.11 & -0.88 & 11.09 \\
$\mathbf{2 . 2}-\mathbf{3}$ & 0.79 & -0.12 & 17.13 \\
$\mathbf{1 . 5}-\mathbf{3}$ & 0.68 & -0.13 & 15.02 \\
\hline
\end{tabular}

Supplementary figure 3.2. Distribution of the LDF in the groups defined by the slice thickness values: 1.5, 2, 2.2 and 3, and pairwise comparisons using Tukey test, no differences were found. 



\section{Chapter 4}

\section{Biological and cognitive correlates of cortical curvature in schizophrenia}

Published in: Psychiatry Research Neuroimaging 2017 Oct 27; 270:68-75. doi: 10.1016/j.pscychresns.2017.10.011. ISSN 09254927.

Impact factor: 2.455. Neuroimaging Q3 (8/14), Psychiatry Q2 (69/142).

Authors: Alba Lubeiro a, Rodrigo de Luis-García b, Margarita Rodríguez c, Aldara Álvarez ${ }^{d}$, Henar de la Red ${ }^{d}$, Vicente Molina a,d,e, ${ }^{*}$
a. Department of Psychiatry, University of Valladolid, Valladolid, Spain.
b. Imaging Processing Laboratory, University of Valladolid, Valladolid, Spain.
c. Radiology Service, University Hospital of Valladolid, Valladolid, Spain.
d. Psychiatry Service, Clinical Hospital of Valladolid, Valladolid, Spain.
e. Neurosciences Institute of Castilla y León (INCYL), University of Salamanca, Spain.
f. CIBERSAM (Biomedical Research Network in Mental Health; Instituto de Salud Carlos III).
* Corresponding author. 


\section{Abstract}

Mean cortical curvature may relate to cortico-cortical connections integrity. We explored the association between prefrontal (PFC) cortical curvature and fractional anisotropy (FA) values for tracts connecting PFC and relevant cortical regions.

In schizophrenia Anatomical and diffusion magnetic resonance images were obtained from 34 patients (16 of them first-episodes) and 32 healthy controls. We calculated curvature at rostral lateral prefrontal (RLPF) and superior medial prefrontal (SMPF) areas and mean FA for the tracts respectively connecting RLPF and SMPF areas with anterior caudal cingulate (ACC), superior temporal gyrus (STG) and superior parietal SP regions. Cognitive and clinical data were collected, including baseline symptoms, clinical global impression change scores from baseline to follow-up, illness duration and treatment dosage.

Patients showed significantly lower FA values in the tracts linking right RLPF-ACC, right SMPF-SPG and bilaterally PFC-STG. FA values mainly in short-range cortico-cortical connections (linking PFC-ACC) were inversely associated with PFC curvature. In patients, cognitive performance was negatively associated with PFC curvature. Larger curvature values were associated with a lack of clinical improvement at follow-up.

We conclude that cortical curvature is influenced by integrity in short cortico-cortical connections and relates to cognition and clinical outcome in schizophrenia patients.

\subsection{Introduction}

The diversity of clinical manifestations of schizophrenia, together with the low replicability of biological findings and the diversity of genetic underpinnings in that syndrome has led to proposals to divide schizophrenia into biologically homogeneous clusters ${ }^{247}$. Recently, hyperdopaminergia ${ }^{151}$, white matter abnormalities ${ }^{35}$ and genetic profiles $^{58}$ have been proposed as biological traits identifying distinct clusters of patients within the schizophrenia syndrome. Moreover, using network measurements and cortical thickness, deficit-type schizophrenia patients showed an increased density of connections relative to non-deficit patients ${ }^{258}$. These findings support the relevance of 
anatomical connectivity for disentangling the variability in biological abnormalities in schizophrenia, since a significant decrease in that parameter might characterize a cluster of patients with a relatively distinct clinical and/or cognitive profile.

In this line, we recently reported the identification of a cluster of schizophrenia patients (including the same proportion of chronic and first episode cases) characterized by larger mean curvature values across most regions of the cortex ${ }^{259}$. This cluster was also different form the rest of cases in other parameters, i.e: i) a lack of the expected increase in striatal glucose metabolism produced by antipsychotics, ii) reduced thalamic and cingulate metabolism and iii) worsening of negative symptoms in the follow-up.

Cortical curvature has been considered a marker related to cortico-cortical connectivity, in particular short-range connections ${ }^{249}$. Tracts connecting nearby areas on the cortical surface have U-fibers (short association fibres with a curve shape), while major tracts (such as inferior and superior longitudinal fascicles) have straight cores ${ }^{260}$. Although this relationship is still a matter of discussion, from that hypothesis can be predicted that white matter deficits would be reflected in higher curvature values ${ }^{232}$. Therefore, the cluster we identified could be also characterized by a deficit of shortrange structural connectivity among relevant cortical regions. It seems thus of interest to test the possible association between cortical curvature and structural connectivity in schizophrenia, which could contribute to further characterize a possible schizophrenia subtype. To do so, we investigated the correlates of cortical curvature in a new schizophrenia sample using diffusion magnetic resonance imaging (dMRI). Cognitive and clinical data were included, and we tested the possible influence of illness duration and antipsychotic treatment upon curvature. We focused on the prefrontal lobe and its more relevant connections, given the previous connectivity and folding alterations reported for this region in schizophrenia $66,261,262$ and in particular the recent identification of white matter alterations in specific tracts connecting this region ${ }^{142}$ and the larger gyrification ${ }^{263}$ or curvature values reported for the same region in schizophrenia ${ }^{259}$. 


\subsection{Subjects and methods}

\subsubsection{Patients}

The sample included 34 schizophrenia patients (16 of them were first-episode (FE) and 33 healthy controls (table 4.1). Participants were fully informed about the study and provided written informed consent. None of these subjects was included in our former report on schizophrenia sub-classification ${ }^{259}$ but they include the subjects that participated in a previous report on structural prefrontal connectivity assessed with dMRI ${ }^{264}$.

Inclusion criteria for patients were schizophrenia diagnosis made by an expert clinician (V.M.) according to the criteria in the Diagnostic and Statistical Manual of Mental Disorders, 5th edition; and for the FE patients, illness duration of less than one year. All patients were on stable doses of atypical antipsychotic treatment. Symptoms were scored using the Positive and Negative Syndrome Scale (PANSS) ${ }^{245}$. Clinical Global Impression change was scored to assess the patient's global improvement, from 1 (large improvement) to 7 (large worsening). Improvement by this method was scored by the treating psychiatrist, when available, after 3 to 6 months of follow-up.

Exclusion criteria were: (i) intelligent quotient under 70; (ii) past or present substance abuse (except caffeine and nicotine); (ii) cranial trauma with loss of consciousness longer than one minute; (iv) for patients, any other mental or neurological diagnosis, and (v) for controls, any current neurological or psychiatric diagnosis or any treatment affecting central nervous system.

The study complied with the ethical standards of the Helsinki Declaration and was approved by the ethical committee of the University Hospital of Valladolid.

\subsubsection{Cognitive assessment}

Global IQ was assessed using a Spanish brief version of the Wechsler Adult Intelligence Scale WAIS-III ${ }^{265}$. We used the Spanish version of Brief Assessment in Cognition in Schizophrenia Scale (BACS) ${ }^{266}$, to assess performance in verbal memory (list learning), working memory (digit span), motor speed (token motor task), verbal fluency (categories), attention and processing speed (symbol coding) and executive function and 
problem solving (Tower of London), as well as the Wisconsing Card Sorting Test (WCST; percent of perseverative errors).

\subsubsection{Diffusion MRI acquisition and processing}

Acquisitions were carried out using a Philips Achieva 3 Tesla MRI unit (Philips Healthcare, Best, The Netherlands) in the MRI facility at Valladolid University, and consisted of T1-weighted and diffusion weighted images.

For the anatomical T1-weighted images, acquisition parameters included: turbo field echo (TFE) sequence, $256 \times 256$ matrix size, $1 \times 1 \times 1 \mathrm{~mm}^{3}$ of spatial resolution and 160 slices covering the whole brain.

With regard to the diffusion weighted images, the parameters of the acquisition protocol were the following: 61 gradient directions, one baseline volume, $b$-value $=1000$ $\mathrm{s} / \mathrm{mm}^{2}, 2 \times 2 \times 2 \mathrm{~mm}^{3}$ of voxel size. $128 \times 128$ matrix and 66 slices covering the entire brain. Total acquisition time was 18 minutes. Both T1 and diffusion weighted images were obtained in the same session and in the same order (T1 followed by diffusion). The total acquisition time refers to the whole T1+DWI protocol.

MRI were processed in order to obtain cortical curvature and connectivity matrices.

From the anatomical images, non-brain structures were first removed using the brain extraction tool from FSL (http://fsl.fmrib.ox.ac.uk) 267. Next, automatic cortical reconstruction was performed using FreeSurfer (http://surfer.nmr.mgh.harvard.edu). From this cortical segmentation, mean radial curvature (defined as the inverse of the radius of the circumference inscribing each gyrus) was calculated, also using FreeSurfer, at superior frontal and rostral middle frontal regions (figure 4.1).

Grey matter, white matter and CSF were also separated, and subcortical grey matter structures were obtained using "fast" and "first" utilities from FSL. respectively ${ }^{268,269}$. These structures were combined to form a "five-tissue-type" image (5tt) using "5ttgen" from MRtrix (www.mrtrix.org). The cortical segmentations provided by Freesurfer were visually inspected. 

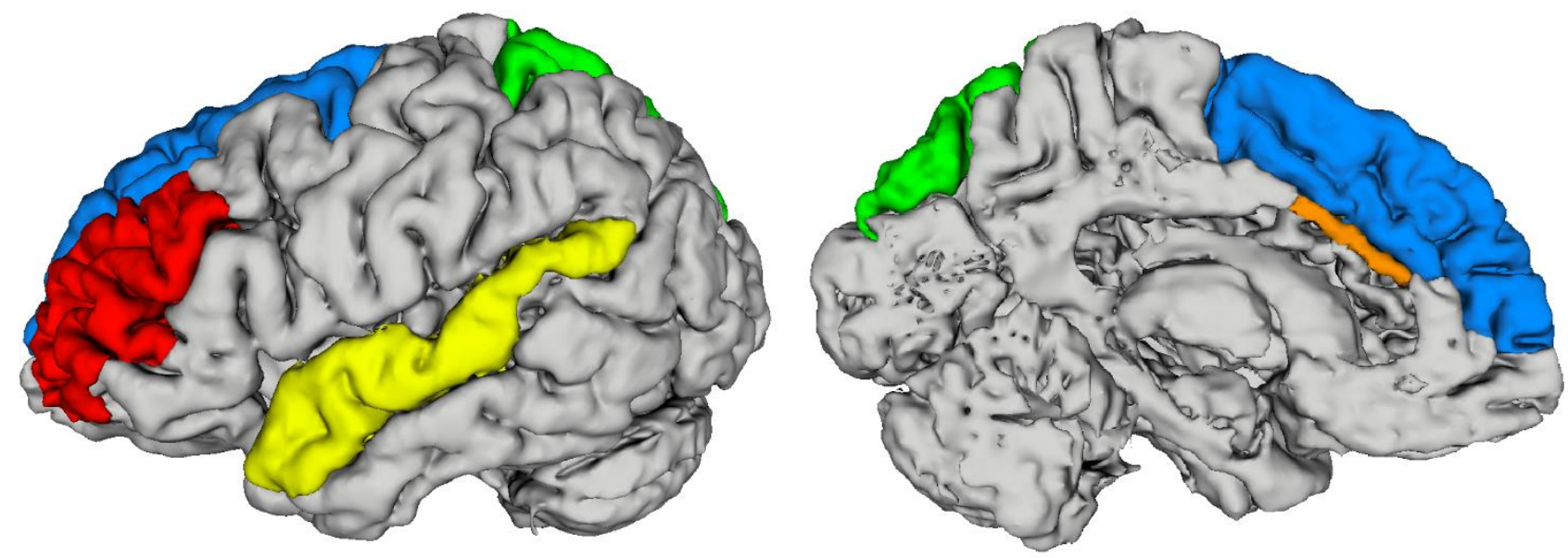

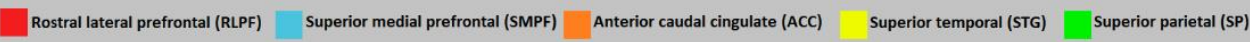

Figure 4.1. Location of the areas selected for cortical curvature (rostral lateral prefrontal (RLPF, in red) and superior medial prefrontal (SMPF, in blue)) and fractional anisotropy (tracts: RLPF- Superior temporal gyrus (STG, in yellow). RLPF- Superior parietal cortex (SP, in green), RLPF-Anterior caudate cingulate cortex (ACC, in orange). SMPF-STG. SMPF-SP and RLPF-ACC) calculation.

From the diffusion weighted images (DWIs), non-nervous tissue components (CSF, meninges, bone) were removed using "dwi2mask" tool from MRtrix ${ }^{270}$. Afterwards, also employing MRtrix, orientation distribution functions were estimated from the diffusion data using spherical deconvolution ${ }^{271}$, and anatomically-constrained tractography was carried out ${ }^{272}$ using the diffusion data and the 5 tt image obtained from the T1-weighted anatomical images (after registration), 2.000.000 streamlines per subject were obtained following this procedure.

In parallel, diffusion tensors at each voxel were estimated using a least squares method ${ }^{273}$, and scalar FA (fractional anisotropy) volumes were computed from the tensor volumes. FA is a well-known descriptor of brain diffusion that has been widely employed in the literature. It measures the amount of anisotropy in the diffusion tensor, that is, how much it deviates from a totally isotropic diffusion. 
Connectome matrices were computed from the tractography output and the (registered) cortical segmentation volumes. $84 \times 84$ connectivity matrices were obtained using mean FA as a connectome metric. A diagram of the processing pipeline is shown in figure 4.2 .

Our primary hypothesis was that FA in short-range connections would show a significant association with frontal curvature values. To evaluate the specificity of this possible relationship we assessed FA in short- (to anterior cingulate) and long-range (to superior parietal and superior temporal gyrus). Thus, from the connectivity matrices, FA was analyzed only in a priori selected pairwise cortico-cortical bilateral connections between superior-medial prefrontal (SMPF) and rostral lateral prefrontal (RLPF) cortex and, on the other hand, caudal anterior cingulate cortex (ACC), superior temporal gyrus (STG) and superior parietal gyrus (SPG) cortex (figure 4.1).

\subsubsection{Statistics}

We compared socio-demographical data (age, sex, education years and parental education) between patients and controls ( $t$-test or $\chi 2$ tests when appropriate).

After testing the normality of data distribution using Kolmogorov-Smirnov and Levene tests, we compared FA and curvature between patients and controls using $t$ tests.

Our main hypothesis was that FA values in the short-range cortico-cortical connections (linking PFC and ACC) would significantly predict mean curvature in the prefrontal region (i.e. the characteristics of those connections would significantly contribute to individual variability in curvature). Therefore, assuming that connectivity would drive variation in curvature, this hypothesis was tested using stepwise multivariate linear regression models in all subjects as well as in patients group, where the independent variables were the FA data. In order to avoid collinearity effects on regression, a principal component analysis (PCA) was first performed using individual FA variables of all subjects for the selected tracts (SMPF and RLPF with ACC, STG and SPG). Eigenvalues higher than the unit plus a scree plot yielded the number of factors to be selected. The resulting individual factor scores were saved. 


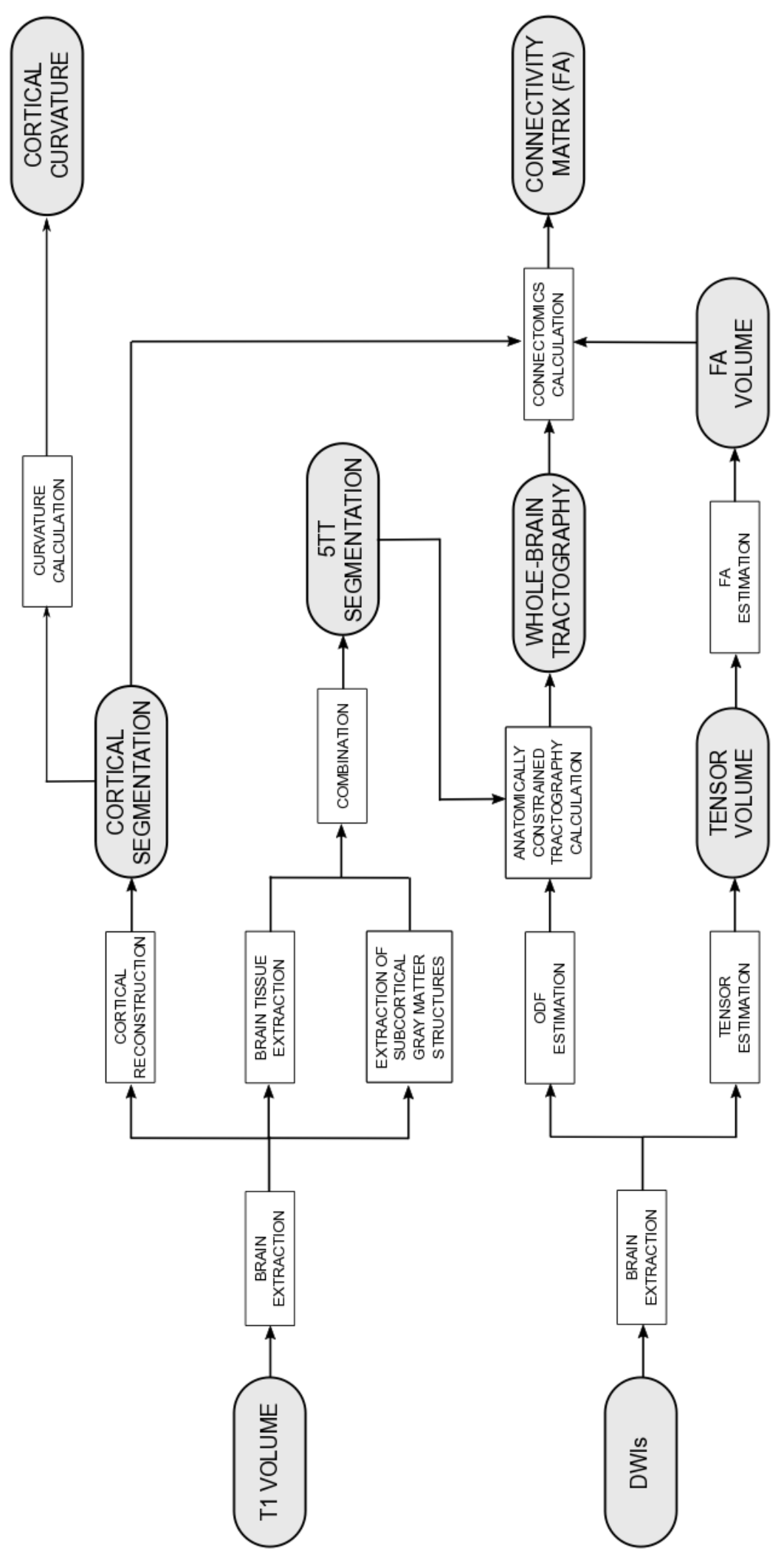

Figure 4.2. MRI processing pipeline applied to obtain cortical curvature and fractional anisotropy. 
We introduced these scores as independent variables in stepwise multivariate linear regression models, where curvature values (SMPF and RLPF, left and right) were the dependent variables. We also tested this association in the FE patients alone to study the possible effects of chronicity.

Furthermore, to summarize cognitive performance, we performed PCA again using cognitive variables from BACS and WCST. Again, individual factor scores were saved after using a scree plot to identify the relevant factors. In this case, since we could not assume a causal role for cognition upon curvature, we assessed the significance of the association between cognitive performance (i.e., individual factor scores) and curvature using Spearman's rho coefficients. This analysis was performed only on patients, since we only expected a significant cognitive deficit in the patients' group.

The relation between duration, symptoms (PANSS scores), treatment dosage $(\mathrm{mg} / \mathrm{d}$ in chlorpromazine (CPZ) equivalents) and curvature was explored using Spearman correlation coefficients.

Finally, we tested the association between curvature and clinical outcome by comparing with U-Mann Whitney tests the curvature values between cases where Clinical Global Impression -change scores were lower than 4 (mild to large improvement) with those with scores equal or larger than 4 (no change or worsening).

\subsection{Results}

\subsubsection{Demographic data}

There were no significant differences between patients and controls in sex $\left(X^{2}=0.16\right.$, $\mathrm{df}=1, p=0.68)$, and age $(\mathrm{t}=-0.51, \mathrm{df}=65, p=0.61)$. Nevertheless, the controls had significantly more years of education than patients $(t=-3.05, d f=57, p=0.003)$, but the difference in parental education between groups was not statistically significant ( $t=-$ 1.28, $\mathrm{df}=56, p=0.21$ ) (table 4.1). 
Table 4.1. Demographic, clinic, cognitive, curvature and FA data are shown for patients, FE and controls. Significant differences with controls: ${ }^{*} p<0.05,{ }^{* *} p<0.01$, $* * * p=0.001$

\begin{tabular}{|c|c|c|c|}
\hline & $\begin{array}{c}\text { Schizophrenia } \\
(n=34) \\
\text { Mean (SD) }\end{array}$ & $\begin{array}{l}\text { First-episode } \\
\qquad(n=16) \\
\text { Mean (SD) }\end{array}$ & $\begin{array}{c}\text { Controls ( } n=33) \\
\text { Mean (SD) }\end{array}$ \\
\hline Age & $34.26(9.98)$ & $28.56(7.77)^{*}$ & $35.61(11.52)$ \\
\hline Sex (M:F) & $21: 14$ & $11: 05$ & $22: 11$ \\
\hline Education (years) & $14.00(3.52)^{* *}$ & $15.25(3.04)$ & $16.62(2.90)$ \\
\hline Parental education (years) & $10.41(4.50)$ & $12.60(4.37)$ & $12.00(4.87)$ \\
\hline Intelligence coefficient & $94.18(11.90)^{* * *}$ & $90.58(11.98)^{* * *}$ & $115.93(10.79)$ \\
\hline Illness duration (months) & $93.59(118.75)$ & $8.62(11.09)$ & - \\
\hline CPZ equivalents (mg) & $396.48(236.55)$ & $393.33(265.33)$ & - \\
\hline PANSS positive & $11.47(3.43)$ & $10.44(2.1)$ & - \\
\hline PANSS negative & $15.63(5.072)$ & $13.88(3.14)$ & - \\
\hline PANSS total & $49.09(12.70)$ & $42.25(6.7)$ & - \\
\hline Verbal memory (L.L) & $35.30(12.12)^{* * *}$ & $37.38(13.74)^{* * *}$ & $50.56(8.98)$ \\
\hline Working memory (D.S) & $16.76(4.41)^{* * *}$ & $18.08(4.58)^{* *}$ & $22.93(2.67)$ \\
\hline Motor speed (M.T) & $61.34(13.45)^{* * *}$ & $61.42(9.44)^{* * *}$ & $83.93(11.02)$ \\
\hline Verbal fluency & $19.30(4.86)^{* * *}$ & $18.56(5.38)^{* * *}$ & $29.24(5.41)$ \\
\hline Attention and processing speed (S.C) & $44.45(15.78)^{* * *}$ & $51.75(12.63)^{* * *}$ & $69.96(15.13)$ \\
\hline Executive function/problem solving (T.L) & $16.14(16.81)$ & $16.17(2.76)$ & $16.81(2.7)$ \\
\hline WCST perseverative errors (\%) & $20.79(17.58)^{* * *}$ & $15.99(9.94)^{* *}$ & $9.46(5.32)$ \\
\hline PCA-F1 & $-0.22(0.92)$ & $0.29(0.61)$ & $0.24(1.06)$ \\
\hline PCA-F2 & $-0.17(1.07)$ & $0.06(0.89)$ & $0.16(0.9)$ \\
\hline printCG1 & $-0.79(0.72)^{* * *}$ & $-0.61(0.55)^{* * *}$ & $0.76(0.51)$ \\
\hline Curvature left RLPF $\left(\mathrm{mm}^{-1}\right)$ & $0.15(0.01)$ & $0.15(0.01)$ & $0.15(0.01)$ \\
\hline Curvature left SMPF $\left(\mathrm{mm}^{-1}\right)$ & $0.13(0.01)$ & $0.13(0.01)$ & $0.13(0.00)$ \\
\hline Curvature right RLPF $\left(\mathrm{mm}^{-1}\right)$ & $0.15(0.01)$ & $0.15(0.01)$ & $0.15(0.01)$ \\
\hline Curvature right SMPF $\left(\mathrm{mm}^{-1}\right)$ & $0.13(0.01)$ & $0.13(0.01)$ & $0.13(0.00)$ \\
\hline FA left RLPF-ACC & $0.41(0.03)$ & $0.42(0.03)$ & $0.42(0.03)$ \\
\hline FA right RLPF-ACC & $0.43(0.04)^{*}$ & $0.44(0.04)$ & $0.45(0.04)$ \\
\hline FA left SMPF-ACC & $0.37(0.04)$ & $0.37(0.04)$ & $0.38(0.04)$ \\
\hline FA right SMPF-ACC & $0.40(0.04)$ & $0.41(0.05)$ & $0.39(0.04)$ \\
\hline FA left RLPF-SP & $0.42(0.03)$ & $0.43(0.02)$ & $0.44(0.02)$ \\
\hline FA right RLPF-SP & $0.43(0.03)$ & $0.44(0.02)$ & $0.44(0.03)$ \\
\hline FA left SMPF-SP & $0.46(0.02)$ & $0.47(0.02)$ & $0.47(0.02)$ \\
\hline FA right SMPF-SP & $0.47(0.02)^{*}$ & $0.48(0.02)$ & $0.48(0.02)$ \\
\hline FA left RLPF-STG & $0.44(0.03)^{*}$ & $0.45(0.02)$ & $0.45(0.02)$ \\
\hline FA right RLPF-STG & $0.45(0.03)^{*}$ & $0.46(0.03)$ & $0.46(0.02)$ \\
\hline FA left SMPF-STG & $0.45(0.02)$ & $0.46(0.02)$ & $0.45(0.02)$ \\
\hline FA right SMPF-STG & $0.46(0.02)^{*}$ & $0.46(0.02)$ & $0.47(0.02)$ \\
\hline
\end{tabular}




\subsubsection{Curvature and FA comparison}

FA and curvature variables were normally distributed in each group $(0.39<Z<1.04$, $0.23<p<1.00$ ). There were no differences in bilateral SMPF or RLPF curvature between patients and controls (table 4.1).

Patients showed significantly lower FA values in right RLPF-ACC $(t=-2.11, d f=61$, $p=0.039)$; right SMPF-SPG ( $\mathrm{t}=-2.01, \mathrm{df}=62, p=0.048)$, bilateral RLPF-STG (left $\mathrm{t}=-2.21$, $\mathrm{df}=62, p=0.031$; right $\mathrm{t}=-2.12, \mathrm{df}=62, p=0.038)$ and right SMPF-STG $(\mathrm{t}=-2.09, \mathrm{df}=62$, $p=0.041$ ) tracts (table 4.1). None of these differences would survive after correction for multiple comparisons.

\subsubsection{FA principal component analyses}

Two main components were selected based on the scree plot and eigenvalues higher than 1 . The first component (PCA-F1) explained $55.56 \%$ of the variance and was directly contributed by long-range cortico-cortical connections (PFC-STG and PFC-SPG). The second component (PCA-F2) explained $10.70 \%$ of the variance and was directly contributed by both PFC-ACC and left PFC-STG connections (table 4.2). Thus, this component is contributed by both short-range and long-range cortico-cortical connections.

Table 4.2. Rotated component matrix for fractional anisotropy PCA. Rotation method: Varimax and Kaiser normalization. Two main components were extracted.

\begin{tabular}{l|c|c}
\hline WM connections & PCA-F1 & PCA-F2 \\
\hline FA left RLPF-ACC & 0.235 & $\mathbf{0 . 8 1 4}$ \\
FA left SMPF-ACC & 0.071 & $\mathbf{0 . 7 3 9}$ \\
FA right RLPF-ACC & 0.281 & $\mathbf{0 . 7 2 1}$ \\
FA right SMPF-ACC & 0.114 & 0.123 \\
FA left SMPF-SP & $\mathbf{0 . 8 7 5}$ & 0.114 \\
FA left RLPF-SP & $\mathbf{0 . 7 3 0}$ & 0.321 \\
FA right SMPF-SP & $\mathbf{0 . 8 3 3}$ & 0.165 \\
FA right RLPF-SP & $\mathbf{0 . 8 0 8}$ & 0.267 \\
FA left SMPF-STG & $\mathbf{0 . 6 4 6}$ & 0.600 \\
FA left RLPF-STG & 0.544 & $\mathbf{0 . 7 1 8}$ \\
FA right SMPF-STG & $\mathbf{0 . 6 8 5}$ & 0.473 \\
FA right RLPF-STG & $\mathbf{0 . 5 9 5}$ & 0.582 \\
\hline
\end{tabular}




\subsubsection{FA PCA prediction of curvature}

In all subjects as a whole, PCA-F2 (contributed by bilateral connections from RLPF and SMPF to anterior cingulate and left RLPF to STG) significantly and inversely predicted bilateral RLPF curvature (table 4.3; figure 4.3a) and right SMPF curvature (table 4.3; figure $4.3 b)$.

In patients, PCA-F2 significantly and inversely predicted bilateral RLPF curvature and right SMPF curvature (table 4.3).

In the FE patients, similar associations were found, PCA-F1 (summarizing long-range connections) significantly and inversely predicted right RLPF curvature and left SMPF curvature (table 4.3). PCA-F2 predicted right SMPF curvature (table 4.3).

In the Controls, PCA-F2 predicted bilateral RLPF curvature (table 4.3).

Since the PCA-F2 component was contributed by FA values in both PFC-cingulate and left PFC-STG tracts, we performed a post-hoc analysis in order to assess the contribution of these tracts to frontal curvature in the patients. To do so, we calculated Pearson's correlation coefficients between FA values in the tracts contributing to the PCA-F2 factor and, on the other side, homolateral RLPF and SMPF curvature values. There was a significant inverse association between left RLPF-STG FA and curvature at left RLPF ( $r=-$ $0.512, p=0.002)$ and SMPF $(r=-0.448, p=0.008)$. Correlation coefficients were also significant for the association between FA in the right RLPF-ACC tract and right RLPF ( $r=-$ $0.424, p=0.014)$ and SMPF $(r=-0.375, p=0.032)$ curvature values. Thus, values in both long-range (prefronto-temporal) and short-range (prefronto-cingulate) contribute to prefrontal curvature in patients.

\subsubsection{Cognitive principal component analyses}

Only one component was selected based on scree plot and eigenvalues higher than 1. This component (printCG1) explained $51.36 \%$ of the variance and was directly contributed by all cognitive variables, except for WCST percentage of perseverative errors that contributed negatively (table 4.4). 

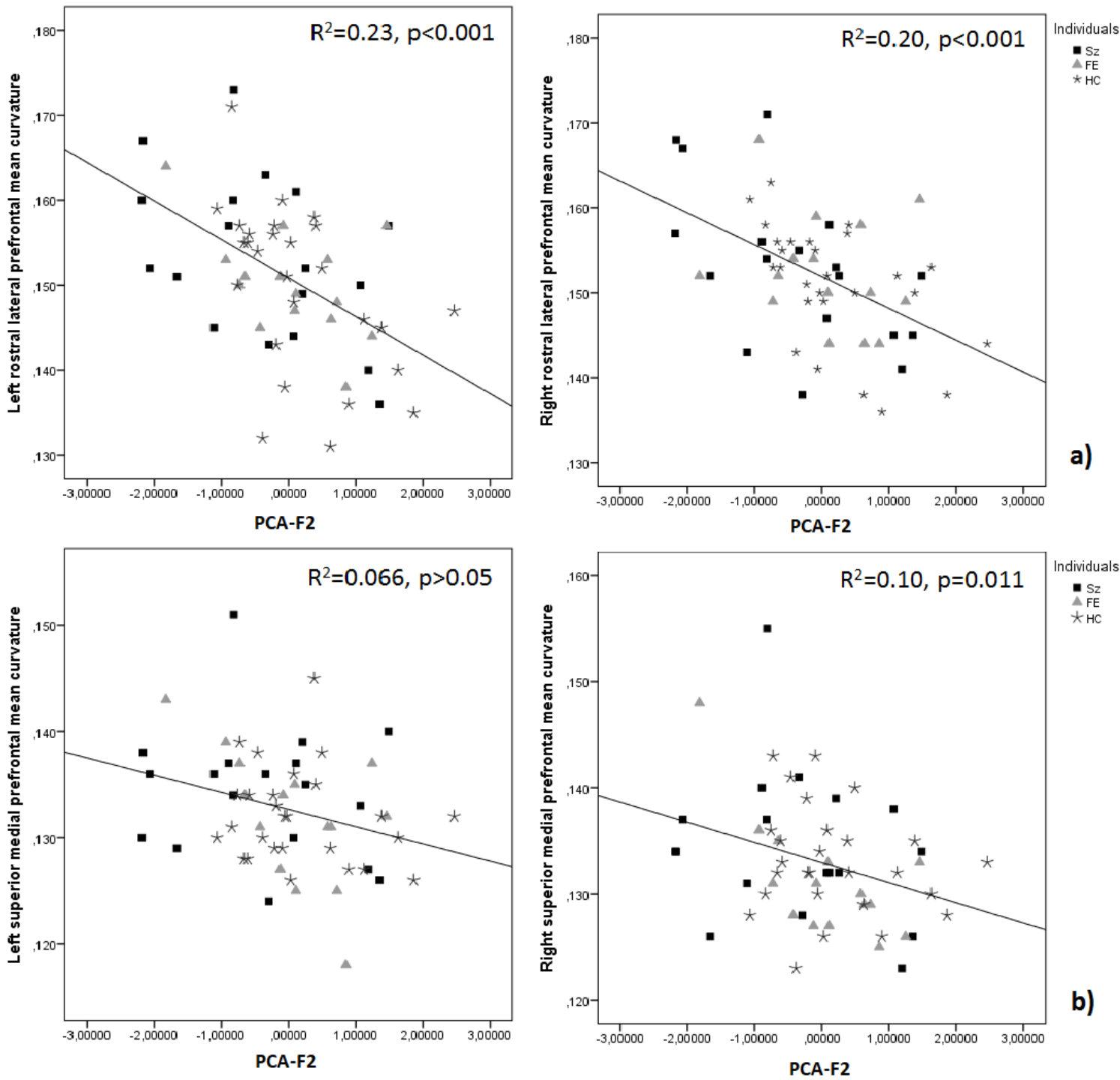

b)
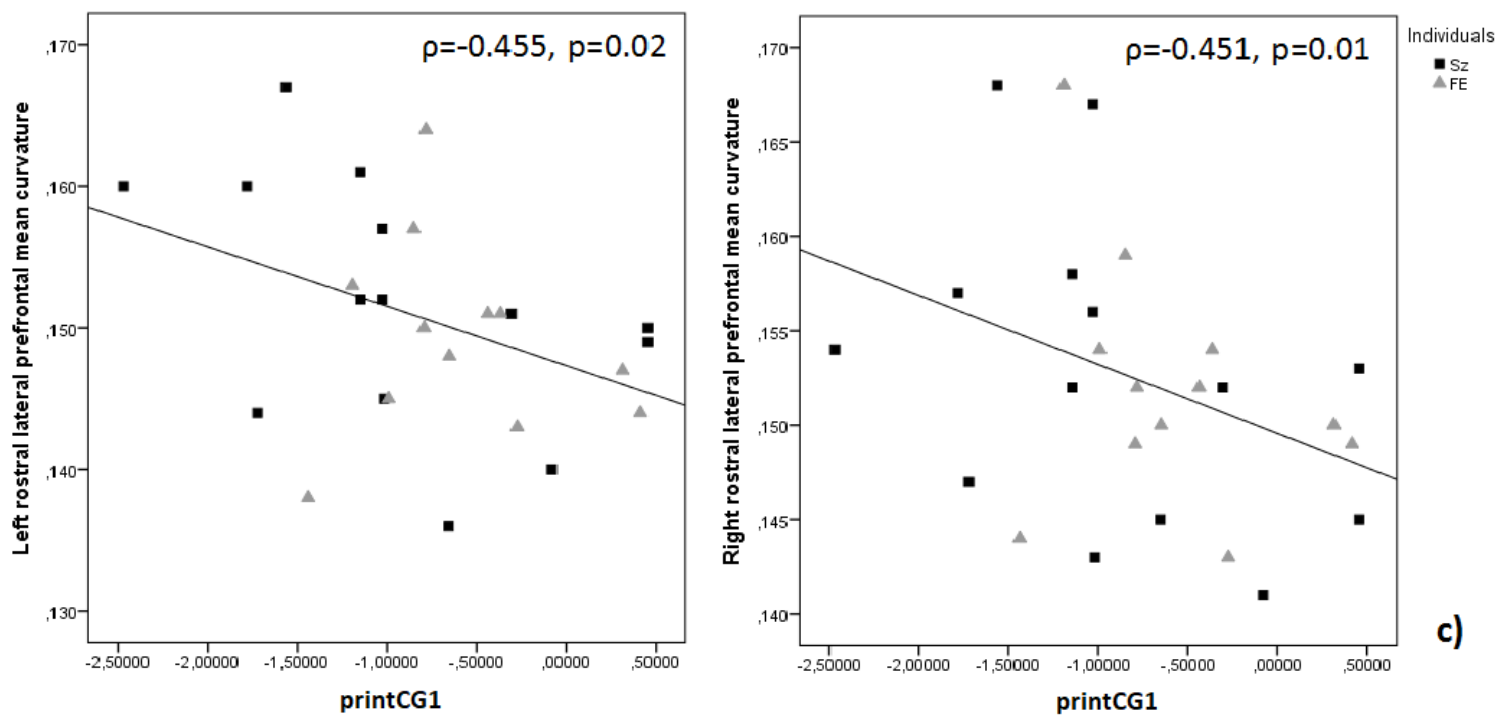

Figure 4.3. Association graphs: a) associations between individual scores in the second factor of the principal component analysis of fractional anisotropy (PCA-F2; see text) and rostral lateral prefrontal (RLPF) curvature for all individuals, b) associations between 
PCA-F2 and SMPF for all individuals, and c) association between individual factor scores in the first component of cognitive performance PCA (printCG1, see text) and RLPF curvature for patients.

Table 4.3. Regression coefficients (R2) between curvature values for the right lateral (RLPF) and superior medial (SMPF) prefrontal regions and the factor scores summarizing FA values respectively for the fronto-parietal and right fronto-temporal tracts (PCA-F1) and for fronto-cingulate and left RLPF-superior temporal tract (PCA-F2). Beta coefficients and $F$ values for the corresponding regression models are also shown. ${ }^{*} p<0.05$; ${ }^{* *} p<0.01 ;{ }^{* * *} p<0.001 ;$ n.s: non-significant

\begin{tabular}{|c|c|c|c|c|c|}
\hline & & RLPF-right & RLPF-left & SMPF-right & SMPF-left \\
\hline PCA-F1 & $\begin{array}{l}\text { All subjects } \\
\text { Patients } \\
\text { First- episode } \\
\text { Controls }\end{array}$ & $\begin{array}{c}\text { n.s. } \\
\text { n.s. } \\
0.27^{*} \beta=-0.55 . \\
F=4.72 \\
\text { n.s. }\end{array}$ & $\begin{array}{l}\text { n.s. } \\
\text { n.s. } \\
\text { n.s. } \\
\text { n.s }\end{array}$ & $\begin{array}{l}\text { n.s. } \\
\text { n.s. } \\
\text { n.s. } \\
\text { n.s. }\end{array}$ & $\begin{array}{c}\text { n.s. } \\
\text { n.s. } \\
0.34^{*} \beta=-0.59 . \\
\mathrm{F}=6.78 \\
\text { n.s. }\end{array}$ \\
\hline PCA-F2 & $\begin{array}{l}\text { All subjects } \\
\text { Patients } \\
\text { First-episode } \\
\text { Controls }\end{array}$ & $\begin{array}{c}0.20^{* * *} \beta=-0.44 . \\
F=14.73 \\
0.20^{* *} \beta=-0.45 . \\
F=7.97 \\
\text { n.s. } \\
0.17 * \beta=-0.41 . \\
F=5.48\end{array}$ & $\begin{array}{c}0.23 * * * \beta=-0.48 \\
F=17.70 \\
0.24^{* *} \beta=-0.49 . \\
F=9.72 \\
\text { n.s. } \\
0.21 * \beta=-0.46 . \\
F=7.03\end{array}$ & $\begin{array}{c}0.10^{*} \beta=-0.32 . \\
F=6.83 \\
0.16^{*} \beta=-0.40 . \\
F=5.97 \\
0.48^{* *} \beta=-0.69 . \\
F=12.05 \\
\text { n.s. }\end{array}$ & $\begin{array}{l}\text { n.s. } \\
\text { n.s. } \\
\text { n.s. }\end{array}$ \\
\hline
\end{tabular}

Table 4.4. Rotated component matrix for cognitive PCA. Rotation method: Varimax and Kaiser normalization. One main component was extracted.

\begin{tabular}{l|c}
\hline & printCG1 \\
\hline Verbal memory & 0.814 \\
Working memory & 0.812 \\
Motor speed & 0.813 \\
Verbal fluency & 0.763 \\
Attention and processing speed & 0.849 \\
Executive function/ problem solving & 0.316 \\
WCST \% of perseverative errors & -0.454 \\
\hline
\end{tabular}




\subsubsection{Cognitive PCA relation to curvature}

In the patients, printCG1 was a significantly and inversely associated with bilateral RLPF curvature (left: $\rho=-0.455, p=0.020$; right: $\rho=-0.451, p=0.021$; figure $4.3 c$ )

\subsubsection{Clinical variables relation to curvature}

Illness duration, treatment dosage and PANSS scores were not significantly associated with frontal curvature.

\subsubsection{Clinical outcome and curvature}

Patients showing no improvement $(n=7)$ showed larger values of left SMPF mean curvature (mean=0.137, $s d=0.004)$ than those improving after follow-up $(n=22$, mean=0.130, $\mathrm{sd}=0.005 ; \mathrm{U}=24.5, \mathrm{z}=-1.98, p=0.04)$. The same was found for the right side, although only at trend level (no improvement: mean=0.136, $s d=0.006$; improvement: mean=0.132, $\mathrm{sd}=0.004 ; \mathrm{U}=1.696, p=0.09$ ).

\subsection{Discussion}

In our patients and controls, mean curvature indices in the frontal lobe were inversely associated with FA values in the tracts connecting this region to anterior cingulum (a nearby cortical area relevant for schizophrenia) and left superior temporal regions. Although our design is cross-sectional and therefore cannot identify causal relationships, this finding is coherent with the proposal that larger mean curvature values (equivalent to larger gyrification), when found in patients, may relate to connectivity deficits through an under-developed expansion of upper cortical layers ${ }^{249}$. However, our data indicate that deficits in connectivity in long-range tracts may also contribute to gyrification alterations in schizophrenia patients. The gyrification process is associated with the formation of proper cortico-cortical connections ${ }^{239}$. Thus, the larger curvature in our cases associated with lower FA values may indicate a developmental deficit. Cerebral cortex gyrification development takes place largely in the third trimester of foetal life ${ }^{248}$, thus our data, in the context of neurodevelopmental theories of schizophrenia, may suggest that some neurodevelopmental arrest in corticocortical connections can underlie cortical curvature increase in at least a subgroup of 
patients. Histopathological data may be necessary to confirm the association between curvature and white matter connectivity deficits in schizophrenia.

Previous data in multiple sclerosis support a similar association to that here found between increased cortical curvature and reduction of both white matter volumes and FA values ${ }^{250}$. Nevertheless, the marked inflammation and scars in white matter usual in multiple sclerosis are absent in schizophrenia, indicating that the underpinnings of the association may differ between these two syndromes. Our results are discrepant with a recent report showing an inverse association between radial diffusivity (RD) and cortical folding (i.e. curvature) in 19 schizophrenia patients ${ }^{274}$. RD increases with WM damage usually are in the opposite direction as FA. Relevant methodological differences may have contributed to the discrepancy since these authors calculated the association between RD at a single voxel located at superior temporal cortex and folding measurement of each vertex of the subjects' surface.

Cortical curvature has genetic underpinnings ${ }^{237}$, therefore, some genetic factors may contribute to a schizophrenia subset where cortico-cortical connections are deficient. Since clinically diverse subgroups within schizophrenia have been proposed on the basis of different genetic underpinnings ${ }^{58}$, it can be speculated that genetic variation can contribute to the differentiation of a cluster within this diagnosis based on cortical curvature.

Our cases did not show significantly larger curvature values as compared to controls, but this finding probably reflects the expected heterogeneity of curvature in a clinicallydefined population of schizophrenia. In fact, in our previous sample, the group comprising most of the cases (89 out of 121) did not show increased curvature values. In the same vein, the lack of FA decrease after correction for multiple comparisons would be coherent with a significant FA deficit in the analysed tracts limited to a subset of cases.

Heterogeneous results regarding curvature have been reported in schizophrenia, with increased $233,275-277$ normal 234,278 and reduced ${ }^{235}$ gyrification indexes being reported. This, along with our former study 259 and the present results support the existence of patients with and without altered cortico-cortical connectivity. Moreover, increased gyrification may also be found in bipolar disorder in the anterior and 
infragenual cingulate cortices ${ }^{263}$, suggesting that this may be a cross-diagnostic alteration. In agreement with this possibility, both bipolar and schizophrenia adolescent patients showed increased sulcal within the frontal lobe, although only in the latter this alteration was found also in the other lobes ${ }^{279}$.

In our sample, we found a similar association between curvature and FA in both FE and chronic patients. In our previous analysis, the cluster with larger curvature was contributed by $50 \% \mathrm{FE}$ and $50 \%$ chronic patients. This suggests that the relation here found is not an artefact of chronicity or treatment. Moreover, in the present sample, neither duration nor current antipsychotic dose was significantly associated with curvature values. Also, in agreement with our previous report, larger curvature values were associated with a worse clinical outcome, which seems coherent with the poor treatment response showed by cases with lower FA values ${ }^{135}$.

We also found an inverse association between prefrontal curvature and cognitive deficits. This gives additional support to the possibility of finding discrete subtypes within schizophrenia diagnosis with or without cognitive deficits associated with frontal connectivity. Previous reports on the association between schizophrenia clusters and structural connectivity measurements did not assess cognition in the participants 35,258,259. However, an association between FA and cognitive deficits has been reported in schizophrenia 280,281 , which is coherent with the direction of our findings. On the other hand, the presence of increased frontal gamma noise power, an electrophysiological measurement reflecting excessive background activity in this band during task performance, distinguished a patients cluster with significant cognitive deficits, which was absent from cases without frontal noise power excess ${ }^{282}$. Although the possibility that structural and functional connectivity might be associated seems logical, functional and anatomical networks connectivity may be dissociated 283,284 , thus functional networks abnormalities may segregate with structural connectivity or contribute to distinct clusters.

The use of tractography for the calculation of the mean FA of the analysed connections may raise some concerns. Several papers ${ }^{285-287}$ have recently provided evidence indicating that current tractography methods produce many false positives, that is, fibre tracts that do not really exist. This lack of specificity would be fatal if global 
network measures obtained from the connectome were being used ${ }^{288}$. However, our analysis only focuses on some specific, well-known connections that are defined a priori, and the tractography method employed does include anatomical constraints designed to limit that problem. Also, these fibre bundles are later used to compute the mean FA across the spatial ROI defined by that bundle. Because of this, the effect of possible false positives would be very limited on the FA values, since the role of tractography in this study is improving the spatial localization of regions on which FA is calculated.

This study has limitations. We could not assess cumulative antipsychotic doses. The sample size is still small, which precluded a clustering analysis similar to that of our previous study and all patients were receiving antipsychotic medication. Although curvature can be stable over the life-span, we cannot rule out that FA values may fluctuate according to clinical state or treatments received, suggesting the interest of longitudinally studies assessing the stability of the relation between curvature and FA here reported. The possible influence of cortical curvature on tractography, by means of its known bias towards gyral crests, cannot be discarded, but this may not have affected mean FA values. Among the study strengths, the study was hypothesis-driven and made use of two different imaging techniques and cognitive measurements. As a conclusion, our results suggest that deficits in both short- and long-range prefrontal connectivity contribute to increased cortical curvature values in schizophrenia, which could help in characterizing distinct subgroups within this syndrome. 


\section{Chapter 5}

\section{Alterations in prefrontal connectivity in}

\section{schizophrenia assessed using diffusion}

\section{magnetic resonance imaging.}

Published in: Progress in Neuro-Psychopharmacology and Biological Psychiatry 2017 Jun 2; 76:107-115. doi: 10.1016/j.pnpbp.2017.03.001. ISSN 02785846.

Impact factor: 4.185 Neurosciences Q2 (66/261), Psychiatry Q1 (28/142).

Authors: Vicente Molina ${ }^{a, b, c, d^{*}}$, Alba Lubeiro ${ }^{a}$, Oscar Soto ${ }^{a}$, Margarita Rodriguez e, Aldara Álvarez ${ }^{b}$, Rebeca Hernández ${ }^{b}$, Rodrigo de Luis-García ${ }^{f}$

a. Department of Psychiatry, University of Valladolid, Valladolid, Spain.

b. Psychiatry Service, Clinical Hospital of Valladolid, Valladolid, Spain.

c. Neurosciences Institute of Castilla y León (INCYL), University of Salamanca, Spain.

d. CIBERSAM (Biomedical Research Network: Mental Health; Instituto de Salud Carlos III).

e. Radiology Service, Clinical Hospital of Valladolid, Valladolid, Spain.

f. Imaging Processing Laboratory, University of Valladolid, Valladolid, Spain.

* Corresponding author. 


\section{Abstract}

Spatial and biological characteristics of structural frontal disconnectivity in schizophrenia remain incompletely understood. Simultaneous streamline count (SC) and fractional anisotropy (FA) analyses may yield relevant complementary information to this end.

Using 3T diffusion magnetic resonance imaging, both SC and FA were calculated at the tracts linking lateral and medial subregions of the prefrontal cortex (PFC) to cingulate, hippocampus, caudate and thalamus in 27 schizophrenia patients (14 firstepisodes) and 27 controls. Relationships of these parameters with cognition, symptoms, treatment doses and illness duration were assessed where significant between-groups differences were detected.

Patients showed lower SC and FA in the tracts linking lateral and medial PFC to the thalamus (likely corresponding to anterior thalamic peduncle) and lower FA in those linking PFC to caudate (likely through internal capsule), right caudal anterior cingulate and left hippocampus (likely corresponding to hippocampal-prefrontal pathway). Moreover, patients showed greater SC values for the tracts linking medial PFC and left caudal anterior cingulate. SC and FA values for the tracts linking PFC and caudal anterior cingulate were positively related to motor speed, executive function, problem-solving and completed categories in WCST. FA for the tract linking right lateral PFC and caudate was directly related to positive symptoms and FA for the tract linking left medial PFC and left thalamus was inversely related to negative symptoms. Treatment doses were not associated with SC or FA values in any tract. Illness duration was negatively associated with SC and FA in the tracts linking PFC and subcortical areas.

As a conclusion, widespread alterations in frontal structural connectivity of PFC can be found in schizophrenia, and are related to cognition, symptoms and illness duration.

\subsection{Introduction}

Disconnectivity in schizophrenia is supported by analyses using diffusion magnetic resonance imaging $(\mathrm{dMRI})$ revealing fractional anisotropy $(\mathrm{FA})$ deficits in white matter 
tracts ${ }^{64,289}$. Available evidence support that such disconnectivity may be particularly evident for the prefrontal cortex (PFC) ${ }^{66,261}$. However, two questions concerning this alteration remain to be clarified. First, which are the specifically affected connections of PFC (i.e. with which particular regions is PFC connectivity more affected). Second, whether connectivity deficits identified in dMRI studies are secondary to impaired myelination or decreased number of axons 290,291 .

In order to investigate these possibilities, dMRI offers potentially useful complementary connectivity descriptors. FA is generally thought to reflect myelin integrity in the corresponding WM tracts, therefore its deficits likely reflecting myelination impairments. Besides, streamline count (SC) quantifies the number of streamlines connecting selected pairs of regions and its value may be associated with the number of axons directly linking these regions ${ }^{292}$. SC is the result of extracting the streamlines connecting two particular regions generated by a whole-brain tractography algorithm. Since the total number of generated streamlines is a fixed parameter, differences in SC values (for instance, between patients and controls) for a certain connection reflect differences in the connection strength of that connection relative to the total amount of connections in the brain. Thus, SC is complementary to FA, since it can provide information about subtle changes in the connectivity pattern that may not be identified using FA alone.

In order to investigate both possibilities (decreased myelination and/or number of axons) and its spatial distribution, we determined both SC and FA for the most relevant connections of the PFC. Since PFC is directly linked to a large number of regions in the normal brain we chose candidate connections by selecting regions directly connected to PFC and also meeting these criteria: i) prior findings supporting its involvement in schizophrenia and ii) having a role in functions deemed to be relevant to this syndrome.

Among the regions strongly linked to the PFC via direct connections and previously reported to be involved in schizophrenia are anterior cingulate ${ }^{293}$, hippocampus ${ }^{294}$, thalamus ${ }^{295}$ and caudate ${ }^{296}$. Besides, these regions play a role in cognitive functions deemed to be altered in schizophrenia, such as working memory, executive functions and social cognition 297,298 . Given the different physiological roles of medial PFC and dorsolateral PFC ${ }^{299}$, and the reported involvement of both medial ${ }^{101,300}$ and lateral PFC 
301,302 in schizophrenia, we studied the tracts connecting both subregions of PFC with the above-mentioned regions of interest.

Therefore, the aim of this study is to analyze both the spatial distribution and characteristics of PFC connectivity alterations in schizophrenia. To this end, the differences between patients and controls in SC and FA in relevant WM tracts were assessed. We hypothesized that deficits in SC and/or FA should be found in patients, independently of their treatment and chronicity and be related to clinical symptoms and/or cognitive deficits.

\subsection{Subjects and Methods}

\subsubsection{Patients}

Our sample included 27 schizophrenia patients (14 of them were first-episode, FE) and 27 age and sex-matched healthy controls (table 5.1). Participants were fully informed about the study and provided written informed consent.

Table 5.1. Demographic and clinical data shown as mean (standard deviation). Firstepisode group ( $n=14)$ is a sub-sample of the schizophrenia patients group ( $n=27)$.

\begin{tabular}{l|c|c|c}
\hline & $\begin{array}{c}\text { Schizophrenia } \\
\boldsymbol{n}=\mathbf{2 7}\end{array}$ & $\begin{array}{c}\text { First episode } \\
\boldsymbol{n}=\mathbf{1 4}\end{array}$ & $\begin{array}{c}\text { Controls } \\
\boldsymbol{n}=\mathbf{2 7}\end{array}$ \\
\hline Age & $33.85(9.13)$ & $29.43(7.94)$ & $33.85(10.54)$ \\
Sex (M:F) & $17: 10$ & $9: 05$ & $18: 09$ \\
Educational level (years) & $13.79(3.56)$ & $15.50(2.78)$ & $15.67(2.50)$ \\
IIIness duration (months) & $88.68(123.84)$ & $7.31(8.30)$ & - \\
CPZ equivalents (mg) & $374.80(193.42)$ & $357.14(215.82)$ & - \\
PANNS Positive & $11.32(3.60)$ & $10.14(2.00)$ & - \\
PANSS Negative & $15.04(5.04)$ & $13.36(2.95)$ & - \\
PANNS Total & $47.60(11.63)$ & $41.00(6.18)$ & - \\
\hline
\end{tabular}

Inclusion criteria for patients were (i) schizophrenia diagnosis according the Diagnostic and Statistical Manual of Mental Disorders, 5th edition criteria; and (ii) for the FE patients, illness duration of less than one year. The diagnoses were made or confirmed by an expert clinician (V.M.). All patients were receiving stable doses of 
atypical antipsychotic treatment at the time of MRI scans. Symptoms were scored using the Positive and Negative Syndrome Scale (PANSS) ${ }^{245}$.

Exclusion criteria were: (i) intelligent quotient under 70; (ii) past or present substance abuse (except caffeine and nicotine); (ii) cranial trauma with loss of consciousness longer than one minute; (iv) for patients, any other mental or neurological diagnosis, and (v) for controls, any current neurological or psychiatric diagnosis or any treatment affecting central nervous system.

The study complied with the ethical standards of the Helsinki Declaration and was approved by the ethical committee of the University Hospital of Valladolid.

\subsubsection{Cognitive assessment}

Global intelligence quotient (IQ) was assessed using a Spanish brief version of the Wechsler Adult Intelligence Scale WAIS-III ${ }^{265}$. Other cognitive assessments included the Spanish version of Brief Assessment in Cognition in Schizophrenia Scale (BACS) ${ }^{266}$ and the Wisconsin Card Sorting Test (WCST; number of categories and percentage of perseverative errors). Direct scores from the subscales of the Spanish version of the BACS were collected, including: verbal memory (list learning), working memory (digit span), motor speed (token motor task), verbal fluency (FAS), attention and processing speed (symbol coding) and problem-solving (tower of London)

\subsubsection{Diffusion MRI acquisition and processing}

Acquisitions were carried out using a Philips Achieva 3 Tesla MRI unit (Philips Healthcare, Best, The Netherlands) at the MRI facility at Valladolid University, including T1-weighted and diffusion-weighted images.

For the anatomical T1-weighted images, acquisition parameters were: turbo field echo (TFE) sequence, $256 \times 256$ matrix size, $1 \times 1 \times 1 \mathrm{~mm}^{3}$ of spatial resolution and 160 slices covering the whole brain.

With regard to the diffusion weighted images, the acquisition protocol parameters were: 61 gradient directions, one baseline volume, $b$-value $=1000 \mathrm{~s} / \mathrm{mm}^{2}, 2 \times 2 \times 2 \mathrm{~mm}^{3}$ of voxel size, $128 \times 128$ matrix and 34 slices covering the entire brain. Total acquisition time was 18 minutes. 
From the anatomical images, non-brain structures were removed in a first step using the brain extraction tool from FSL (http://fsl.fmrib.ox.ac.uk) ${ }^{267}$. Next, automatic cortical reconstruction was performed using FreeSurfer (http://surfer.nmr.mgh.harvard.edu). Grey matter, white matter and CSF were also separated, and subcortical grey matter structures were obtained using "fast" and "first" utilities from FSL, respectively 268,269. These structures were combined to form a "five-tissue-type" image (5tt) using "5ttgen" from MRtrix (www.mrtrix.org).

From the diffusion weighted images (DWIs), brain was then extracted using "dwi2mask" tool from MRtrix v3.12 270. Afterwards, employing MRtrix, orientation distribution functions were estimated from the diffusion data using spherical deconvolution ${ }^{271}$, and anatomically-constrained tractography was carried out ${ }^{272}$ using the diffusion data and the 5tt obtained from the T1-weighted anatomical images (after registration), setting the number of streamlines per subject at 2.000.000.

In parallel, diffusion tensors at each voxel were estimated using a least squares method 273 and scalar FA volumes were computed from the tensor volumes. FA measures the amount of anisotropy in the diffusion tensor, that is, how much it deviates from a totally isotropic diffusion.

Afterwards, connectome matrices were computed from the tractography output and the (registered) cortical segmentation volumes. $84 \times 84$ connectivity matrices were obtained using (i) FA and (ii) streamline count as connectome metrics. A diagram of the processing pipeline is shown in figure 5.1.

From the connectivity matrices, further analysis was performed only in a priori selected pairwise connections between superior-medial prefrontal (SMPF) and rostral lateral prefrontal (RLPF) cortex and, on the other hand, caudal anterior cingulate cortex (CACC), hippocampus, thalamus and caudate (figure 5.2). Only homolateral connections were assessed. 


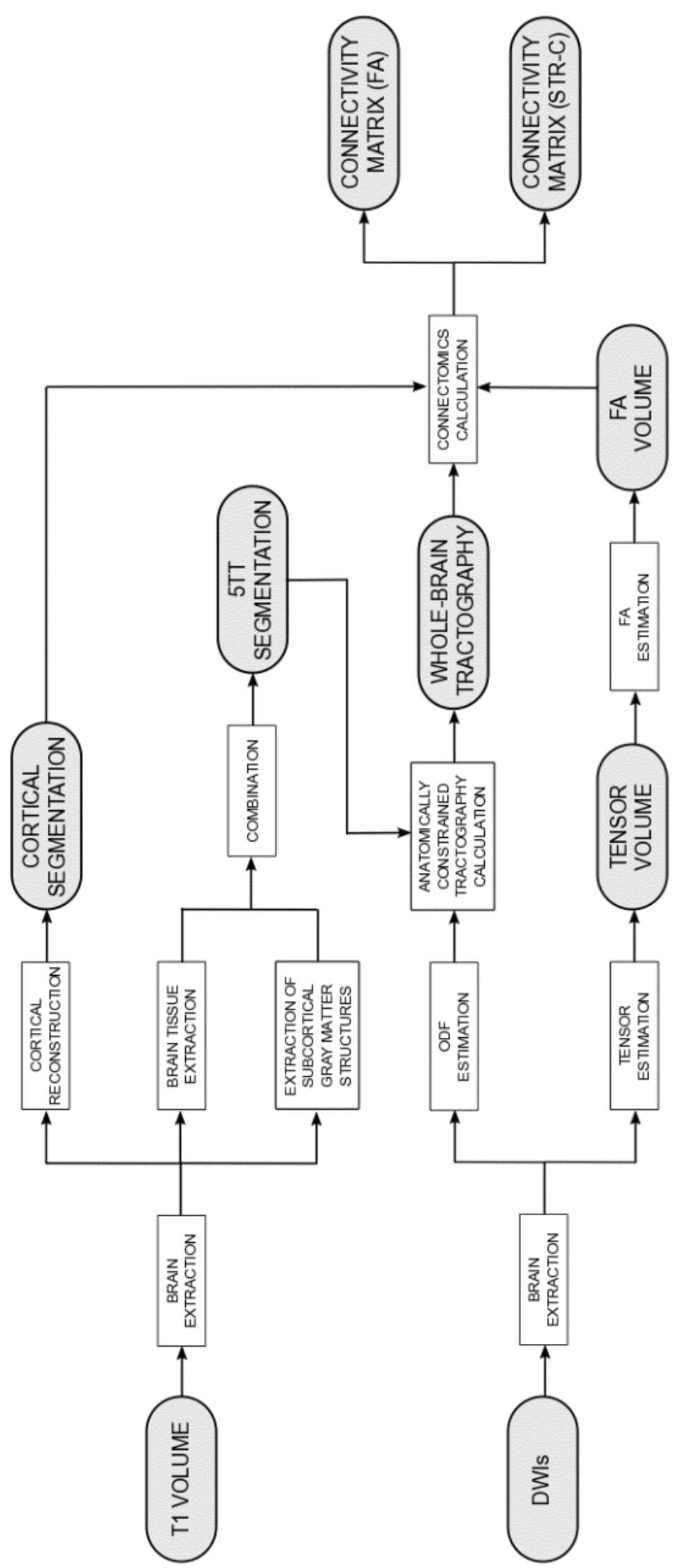

Figure 5.1. Processing pipeline yielding streamline count and fractional anisotropy values. 

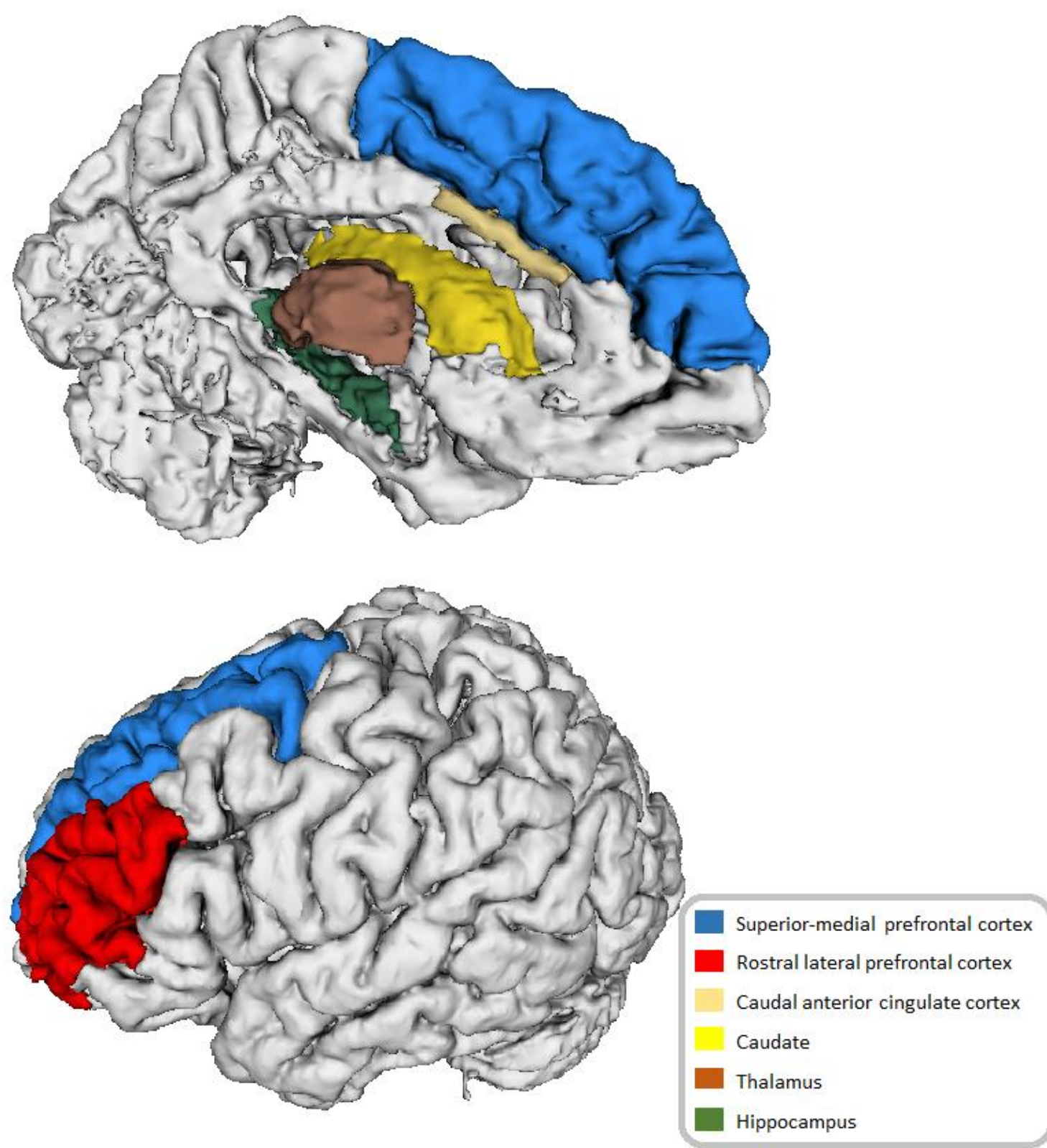

Figure 5.2. Anatomical location of the areas selected for the analyses.

\subsubsection{Statistical analyses}

The statistical significance of between-groups differences in age, sex and years of education was assessed using chi-squared or $t$-tests.

After testing normal distribution (using Kolmogorov-Smirnov tests) of dMRI data, the main hypothesis was tested by assessing the significance of between-groups differences in SC and FA for the tracts linking SMPF and RLPF to the selected regions using $t$-tests 
for independent samples. Since we tested an a priori hypothesis, significance level was set at $p<0.05$. We also assessed the relationship between SC and FA in the tracts where significant between-group differences were found in the former analysis by calculating Pearson's r coefficients between these values.

The contribution of the connectivity alterations to cognitive and clinical variation was assessed using stepwise multivariate linear regressions with SC and FA values as independent variables and cognitive and PANNS scores as dependent variables. Possible associations between illness duration (in months), treatment dose (in milligrams of chlorpromazine equivalents) and SC and FA values was similarly assessed.

Finally, we compared SC and FA values between FE patients and controls using nonparametric tests (U-Mann Whitney) and the relationship between SC and FA data and symptoms and cognition in that group was assessed using Spearman's rho coefficients.

\subsection{Results}

\subsubsection{Demographic data}

No significant differences were found between patients and controls in age $(t=0.00$, $\mathrm{df}=52, p=1.00)$, sex $\left(\mathrm{X}^{2}=0.081, \mathrm{df}=1, p=0.776\right)$ or years of education $(\mathrm{t}=-1.378, \mathrm{df}=21$, $p=0.183$ ) (table 5.1).

\subsubsection{Connectivity data}

\subsubsection{Streamline count}

$S C$ values were normally distributed $(Z<1.301, d f=27, p>0.05)$. Significant differences between groups were found for SC linking the following pairs of regions: left SMPFCACC, with higher values in patients $(\mathrm{t}=2.795, \mathrm{df}=52, p=0.007)$; right RLPF-thalamus ( $\mathrm{t}=-$ 2.011, $\mathrm{df}=52, p=0.049)$ and right SMPF-thalamus ( $\mathrm{t}=-2.731, \mathrm{df}=52, p=0.009)$. with lower values in patients (table 5.2). 
Table 5.2. SC and FA values for each pair of regions, shown as mean (sd). The statistically significant differences between patients and healthy controls are highlighted: ${ }^{*} p<0.05 ;{ }^{* *} p<0.01 ; * * p<0.001$.

\begin{tabular}{|c|c|c|c|}
\hline & $\begin{array}{l}\text { Schizophrenia } \\
\qquad n=27\end{array}$ & $\begin{array}{l}\text { First-episode } \\
\qquad n=14\end{array}$ & $\begin{array}{c}\text { Controls } \\
\qquad n=27\end{array}$ \\
\hline \multicolumn{4}{|l|}{ Streamline count (SC) } \\
\hline Left RLPF-CACC & $402.89(442.07)$ & $533.29(539.54)$ & $299.19(223.96)$ \\
\hline Left SMPF-CACC & $3806.96(1551.75)^{* *}$ & $4075.64(1452.94)^{* *}$ & $2756.81(1185.21)$ \\
\hline Right RLPF-CACC & $401.63(332.27)$ & 453.79 (362.44) & $386.81(249.48)$ \\
\hline Right SMPF-CACC & 2643.37 (999.98) & 2870.07 (790.67) & $3029.11(947.52)$ \\
\hline Left RLPF-hippocampus & $36.22(49.43)$ & $28.21(20.24)$ & $59.26(61.9)$ \\
\hline Left SMPF-hippocampus & $94.3(91)$ & $116.57(118.6)$ & $161.37(177.31)$ \\
\hline Right RLPF-hippocampus & $45.04(47.39)$ & $39.57(46.29)$ & $32.33(29.53)$ \\
\hline Right SMPF-hippocampus & $86.22(54.77)$ & $92.93(57.57)$ & $89.93(74.01)$ \\
\hline Left RLPF-thalamus & 914.41 (519.49) & $963.93(601.16)$ & $1038.37(748.89)$ \\
\hline Left SMPF-thalamus & $3495.41(1733.25)$ & $3963.64(1942.81)$ & 3505.96 (1354.35) \\
\hline Right RLPF-thalamus & $713.41(505.81)^{*}$ & $810.64(514.23)$ & $1054.59(721.88)$ \\
\hline Right SMPF-thalamus & $2512.85(1148.75)^{* *}$ & $2899.93(1150.11)$ & 3574.63 (1661.99) \\
\hline Left RLPF-caudate & $3077.81(1256.13)$ & $3114.86(1420.14)$ & $3001.63(1333.65)$ \\
\hline Left SMPF-caudate & $3528.33(1469.58)$ & $3421.86(1668.19)$ & 3315.19 (1248.65) \\
\hline Right RLPF-caudate & 2963.11 (1090.69) & $3114.36(1151.36)$ & 2849.78 (1159.92) \\
\hline Right SMPF-caudate & $4453.63(2028.34)$ & $4512.93(2458.78)$ & $4415.81(1745.8)$ \\
\hline \multicolumn{4}{|l|}{ Fractional anysotropy (FA) } \\
\hline Left RLPF-CACC & $0.41(0.04)$ & $0.42(0.03)$ & $0.42(0.03)$ \\
\hline Left SMPF-CACC & $0.37(0.05)$ & $0.37(0.04)$ & $0.38(0.04)$ \\
\hline Right RLPF-CACC & $0.43(0.04)$ & $0.44(0.04)$ & $0.45(0.04)$ \\
\hline Right SMPF-CACC & $0.41(0.04)$ & $0.41(0.05)$ & $0.40(0.04)$ \\
\hline Left RLPF-hippocampus & $0.45(0.03)$ & $0.45(0.03)$ & $0.46(0.04)$ \\
\hline Left SMPF-hippocampus & $0.48(0.04)^{*}$ & $0.49(0.04)$ & $0.50(0.03)$ \\
\hline Right RLPF-hippocampus & $0.45(0.03)$ & $0.46(0.03)$ & $0.46(0.04)$ \\
\hline Right SMPF-hippocampus & $0.49(0.04)$ & $0.50(0.04)$ & $0.49(0.04)$ \\
\hline Left RLPF-thalamus & $0.44(0.02)$ & $0.44(0.03)$ & $0.45(0.03)$ \\
\hline Left SMPF-thalamus & $0.46(0.02)^{*}$ & $0.47(0.02)$ & $0.48(0.03)$ \\
\hline Right RLPF-thalamus & $0.44(0.02)$ & $0.45(0.02)$ & $0.45(0.02)$ \\
\hline Right SMPF-thalamus & $0.47(0.03)$ & $0.47(0.03)$ & $0.48(0.02)$ \\
\hline Left RLPF-caudate & $0.36(0.03)$ & $0.37(0.03)$ & $0.37(0.03)$ \\
\hline Left SMPF-caudate & $0.41(0.03)^{*}$ & $0.42(0.03)$ & $0.43(0.03)$ \\
\hline Right RLPF-caudate & $0.36(0.03)^{*}$ & $0.37(0.03)$ & $0.38(0.03)$ \\
\hline Right SMPF-caudate & $0.41(0.02)$ & $0.41(0.02)$ & $0.42(0.03)$ \\
\hline
\end{tabular}




\subsubsection{Fractional anisotropy}

FA variables were normally distributed $(Z<0.484, d f=27, p>0.70)$. FA values in tracts between the following pairs of regions were significantly lower in patients: right RLPFCACC ( $\mathrm{t}=-2.280, \mathrm{df}=52, p=0.027)$; right RLPF- caudate ( $\mathrm{t}=-2.297, \mathrm{df}=52, p=0.026)$; left SMPF-hippocampus ( $\mathrm{t}=-2.270, \mathrm{df}=52, p=0.027)$; left SMPF-thalamus $(\mathrm{t}=-2.413, \mathrm{df}=52$, $p=0.019)$; left SMPF-caudate $(\mathrm{t}=-2.291, \mathrm{df}=52, p=0.026)$ (table 5.2).

\subsubsection{Relation between $\mathrm{SC}$ and FA}

There was a significant direct association between SC values for the tracts linking right RLPF and thalamus and FA values for all the tracts showing significantly decreased FA values in the patients $(0.569<r<0.446 ; 0.002<p<0.02 ; n=27)$.

\subsubsection{Relations of SC and FA with clinical and cognitive data}

The following significant associations were detected between variables showing significant between groups differences and clinical and cognitive data: i) SC between left SMPF and CACC and was directly related to motor speed $\left(R^{2}=0.295, p=0.009, \beta=0.543\right)$;

ii) FA between right RLPF and CACC was directly related to problem solving $\left(R^{2}=0.241\right.$, $p=0.02, \beta=0.491)$ and completed categories in WCST ( $\left.R^{2}=0.256, p=0.019, \beta=0.506\right) ;$ iii) FA between right RLPF and caudate was directly related to positive symptoms $\left(R^{2}\right.$ $=0.171, p=0.04, \beta=0.413)$; iv) FA between left SMPF and thalamus was inversely related to negative symptoms $\left(R^{2}=0.165, p=0.044, \beta=-0.406\right.$; figure 5.3$)$.

\subsubsection{Effect of illness duration and treatment}

Illness duration inversely predicted SC between right SMPF and thalamus $\left(R^{2}=0.239\right.$, $p=0.013, \beta=-0.489) ;$ FA between left SMPF and hippocampus $\left(R^{2}=0.199, p=0.025, \beta=-\right.$ $0.446)$ and FA between left SMPF and caudate $\left(R^{2}=0.232, p=0.015, \beta=-0.481\right.$, figure 5.4). Treatment doses were not associated with any of dMRI values with differences between groups. 

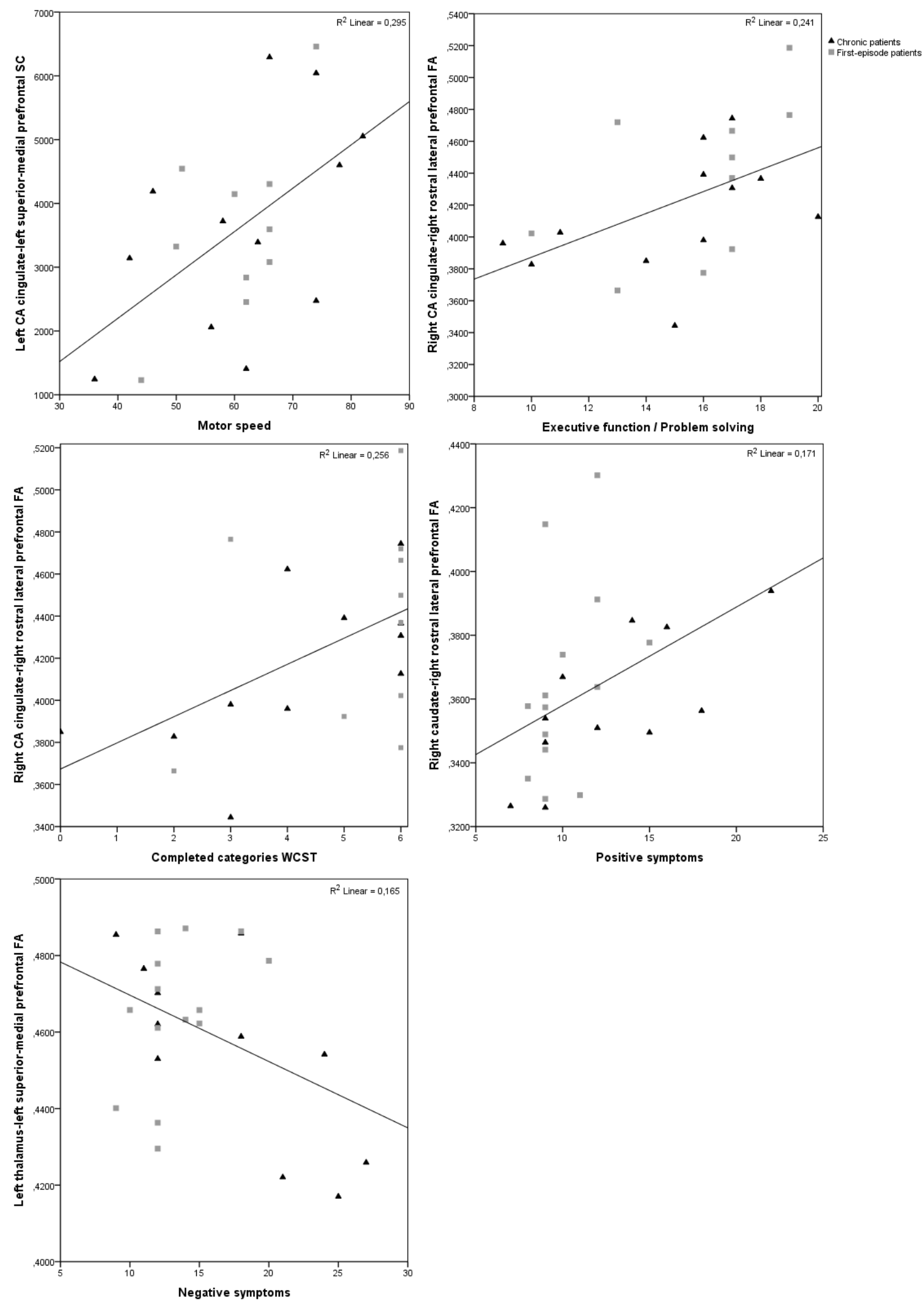

Figure 5.3. Scatterplots showing the significant associations between regional connectivity values and clinical scores. Black triangles = chronic patients, grey squares= first-episode patients. 

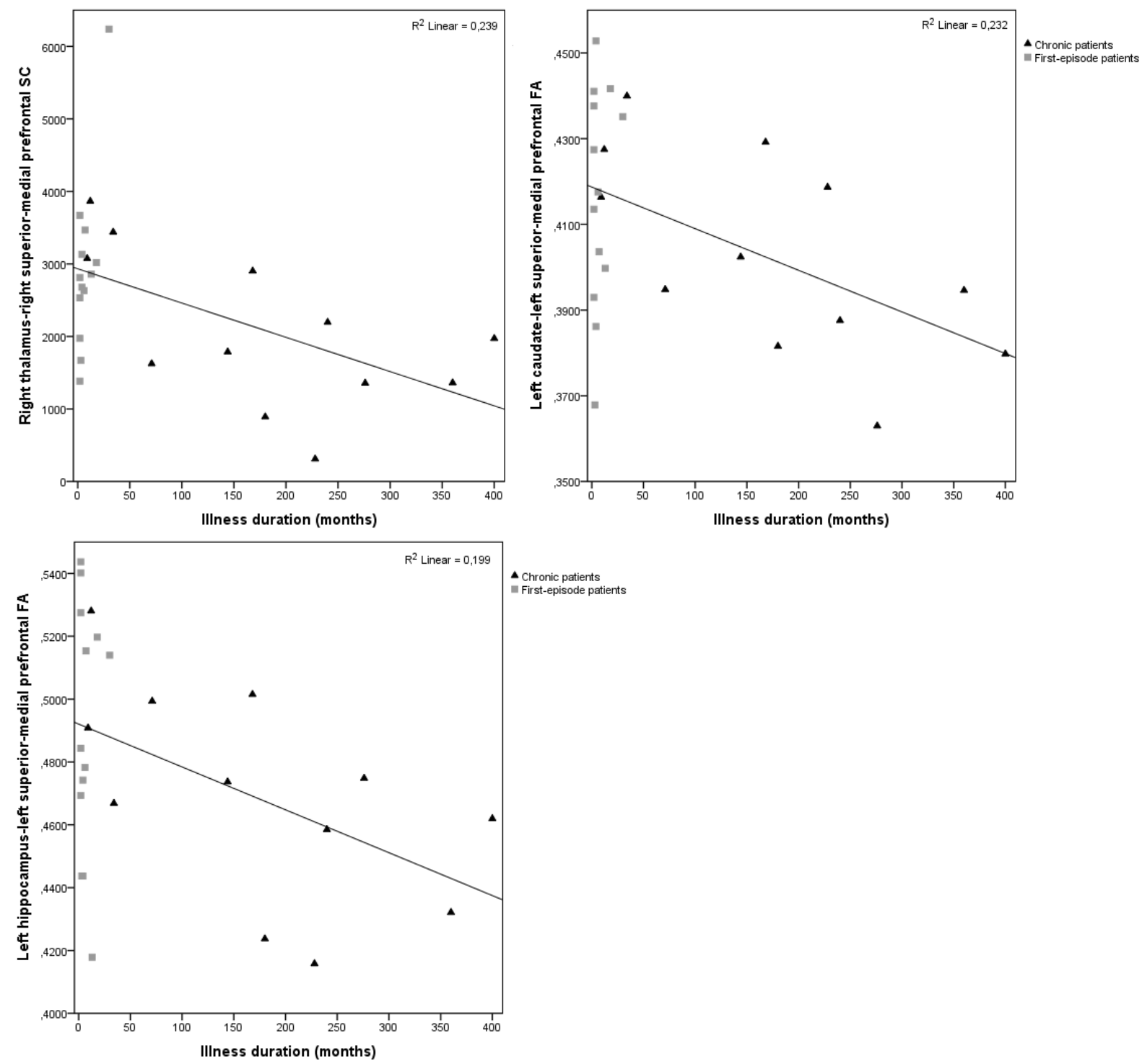

Figure 5.4. Scatterplots showing the significant associations between regional connectivity values and illness duration. Black triangles = chronic patients, grey squares= first-episode patients.

\subsubsection{Comparison between first episode and controls}

There were no significant differences between FE patients and controls in age $(\mathrm{U}=-$ 140.00, $p=0.178)$, sex $\left(X^{2}=0.054, d f=1, p=0.541\right)$ or years of education $(U=34.00$, $p=0.844)$. There were significant differences between FE patients and controls in SC between left SMPF and CACC $(U=92, Z=-2.667, p=0.008)$, FE showing higher values. There were not significant FA differences between those groups. 


\subsection{Discussion}

Our data reveal a significant alteration of FA and/or SC in most of the selected connections between PFC and target regions relevant to schizophrenia. Alterations in FA were more widespread, suggesting that myelination deficits may be more prevalent in these connections than deficits in the number of axons, which might be limited to PFCthalamic tracts. In anatomical terms, this connection, probably correspond to the anterior thalamic peduncle, the anterior part of the thalamic radiation. Both parameters showed similar relationships to cognitive deficits and symptoms.

The deficit in the number of streamlines connecting right medial and lateral PFC and thalamus is coherent with a decreased number of axons in the WM tracts linking these regions. Although we cannot fully discard that the lower SC values in the patients might be secondary to myelination deficits in these tracts, this seems unlikely given its normal FA values. FA was instead significantly lower in the left PFC-thalamic connection, where it was inversely related to negative symptoms scores. Taken together, these data support a role in schizophrenia for a decreasing number of fibres and myelination deficits in tracts connecting thalamus and PFC. These regions have a common embryonic origin ${ }^{303}$, which may be coherent with neurodevelopmental theories in schizophrenia.

Previous results agree with such a possible alteration in structural PFC-thalamic connectivity. Decreased FA was reported in the anterior limb of internal capsule, which also contains fibre tracts from medial dorsal thalamus to frontal cortex ${ }^{304-306}$. Using dynamic causal modelling and FA, altered connectivity was found between the prefrontal cortex, thalamus and anterior cingulate in chronic patients ${ }^{307}$. The possible alteration in fronto-thalamic connections is also consistent with meta-analytic data showing lower FA in schizophrenia patients in frontal lobe WM (including fibres connecting frontal, cingulate cortex and thalamus ${ }^{64}$. A posterior meta-analysis reported decreased FA values in anterior thalamic radiation (including the anterior thalamic peduncle), cingulum, fornix, inferior longitudinal fasciculi and inferior frontal occipital fasciculi ${ }^{31}$.

The significant association in our patients between altered SC values in the PFCthalamic connection and other FA values found to be abnormal in the patients (with the 
hippocampus and caudate, likely travelling through internal capsule) suggests that common factors may be driving both changes.

More intriguingly, a higher SC value was found in the patients in the connection between left CACC and SMPF, being directly associated with motor speed. A similar increase was described in FE patients ${ }^{308}$ and increased connectivity has been proposed to relate to psychotic symptoms and to neurodevelopmental anomalies perhaps via abnormal pruning ${ }^{290}$. In this line, patients suffering from hallucinations showed higher FA values in the left cingulum bundle ${ }^{309}$ and FA values in the arcuate fasciculi were directly correlated to hallucinations scores ${ }^{310}$. Considered together, this suggests the possibility of a hampered cortico-subcortical connectivity in schizophrenia (with thalamus, caudate and hippocampus) along with an excess of cortico-cortical connections, at least with relatively near regions, such as anterior cingulate.

In our patients, prefrontal connectivity was directly associated with cognitive performance. Similar associations were described in never-medicated chronic ${ }^{311}$, chronic medicated ${ }^{281}$ and adolescent-onset ${ }^{312}$ patients. Similarly, a positive relation between FA in the cingulum and working memory, along with a negative relation to errors in the WCST has been reported ${ }^{313}$, as well as a relationship between impaired fronto-temporal and cortico-subcortical connections and deficits in executive and motor function ${ }^{308}$.

FA was also associated in our cases to positive (directly) and negative (inversely) symptoms. Consistently with this, a positive relation between FA and positive symptoms was reported in FE or recent onset schizophrenia ${ }^{314-316}$ and in chronic patients ${ }^{317}$. However, other studies described no relation between diffusion parameters and symptoms 281,311,318-320. Regarding negative symptoms, our data are coherent with reported associations between higher negative symptoms and lower FA values in thalamic radiation, corpus callosum, inferior fronto-occipital fasciculus and inferior longitudinal fasciculus ${ }^{35}$, and in the fronto-occipital fasciculus ${ }^{316}$. These discrepancies may suggest that different clinical profiles might be characterized by distinct connectivity patterns. In this line, decreased FA in left uncinated fasciculus was reported in deficit schizophrenia ${ }^{321}$. Moreover, within FE schizophrenia patients, one subgroup 
could be characterized by widespread WM anomalies and higher negative symptoms ${ }^{35}$, and patients with poor treatment response showed lower FA than good-responders ${ }^{135}$.

Our FE group did not show the same pattern of SC and FA differences that the chronic patients showed in comparison to controls. Although this lack of significance might have been contributed by the smaller number of FE patients, the inverse correlation between illness duration and SC and FA values suggests that deficits in these parameters may become significant in later stages of illness. This could agree with the larger FA reductions were reported in chronic than in FE patients ${ }^{322}$ and the larger percent of WM volume loss in early phases of schizophrenia ${ }^{323}$. Moreover, perhaps only a subset of FE shows FA deficit ${ }^{35}$, which may explain in part why this was not evident in our cases. This heterogeneity could justify discrepancies with meta-analytic evidence in FE reporting FA decreased mainly in fronto-limbic connections ${ }^{93}$, and reviews suggesting decreased FA and mean diffusivity in this and other tracts ${ }^{324}$.

The association found between connectivity and illness duration agrees with the results of a meta-analysis ${ }^{31}$ and more recent data ${ }^{258,325}$. In this context, the lack of association between antipsychotic doses and diffusion parameters suggests that the effect of illness duration upon connectivity is not a direct effect of longer exposure to antipsychotics. Some results suggest that antipsychotics may instead increase FA, perhaps through a myelinating effect ${ }^{135,326}$.

Our study has limitations. The sample size is relatively small and all the patients were medicated. Other tracts not assessed in the present study may show relevant results in schizophrenia. The exact meaning of SC changes is incompletely understood, and may be considered an indirect estimation of numbers of axons.

As a conclusion, prefrontal connectivity may be hampered in schizophrenia and this may play a role in cognitive and clinical deficits. Complementary SC and FA data may help in elucidating the characteristics of that alteration. 


\section{Chapter 6}

\section{Variation at NRG1 genotype related to}

\section{modulation of small-world properties of}

\section{the functional cortical network}

Published in: European Archives of Psychiatry and Clinical Neuroscience 2017 Feb;267(1):25-32 1 doi: 10.1007/s00406-015-0659-0. ISSN 09401334 .

Impact factor: 3.617. Psychiatry Q2 (41/142).

Authors: Alba Lubeiro ${ }^{a}$, Javier Gomez-Pilar ${ }^{b}$, Oscar Martín ${ }^{a}$, Aitor Palominoc, Myriam Fernández ${ }^{d}$, Ana González-Pinto ${ }^{d}$, Jesús Poza ${ }^{b, f}$, Roberto Hornero ${ }^{b, f}$, Vicente Molina ${ }^{a, e, f, g *}$
a. Department of Psychiatry, University of Valladolid, Valladolid, Spain.
b. Biomedical Engineering Group, Department TSCIT, University of Valladolid, Spain.
c. Neurotek, Neurosciences Department, University of Basque Country, Vizcaya, Spain.
d. Psychiatry Department, Hospital Universitario de Álava, Vitoria-Gasteiz, Álava, Spain.
e. Psychiatry Service Clinical University Hospital of Valladolid, Valladolid, Spain.
f. Neurosciences Institute of Castilla y León (INCYL), University of Salamanca, Spain.
g. CIBERSAM (Biomedical Research Network: Mental Health; Instituto de Salud Carlos III).
* Corresponding author. 


\section{Abstract}

Functional brain networks possess significant small-world (SW) properties. Genetic variation relevant to both inhibitory and excitatory transmission may contribute to modulate these properties.

We hypothesized that in healthy controls, genotypic variation in Neuregulin 1 (NRG1) related to the risk of psychosis (risk-alelles) would contribute to functional SW modulation of the cortical network.

Electroencephalographic (EEG) activity during an odd-ball task was recorded in 144 healthy controls. Then, Small-worldness index (SWn) was calculated in five frequency bands (i.e. theta, alpha, beta1, beta 2, and gamma) for baseline (from -300 to the stimulus onset) and response (150 to 450 ms post-target stimulus) windows. The SWn modulation was defined as the difference in SWn between both windows. Association between SWn modulation and carrying the risk allele for three single nucleotide polymorphisms (SNP) of NRG1 (i.e. rs6468119, rs6994992, and rs7005606) was assessed.

A significant association between three SNPS of NRG1 and the SWn modulation was found, specifically: NRG1 rs6468119 in alpha and beta-1 bands; NRG1 rs6994992 in theta band; and NRG1 rs7005606 in theta and beta-1 bands.

In conclusion, genetic variation at NRG1 may influence functional brain connectivity through the modulation of SWn properties of the cortical network.

\subsection{Introduction}

Mental activity is likely contributed by the coordinated activity of different cortical regions ${ }^{85,327,328}$. These brain networks show small-world (SW) properties, characterized by high local clustering and low average distance between nodes (low characteristic path length). SW architecture balances segregation (i.e., the ability of a network to work locally) and integration (i.e., the capacity to communicate separated brain areas) in functional brain networks. These properties can be quantified using a network 
parameter: the small-worldness $(S W n)^{115,116,329}$, whose heritability has been proposed to be large ${ }^{330}$.

SWn of brain network properties can be studied using structural (anatomical (MRI) or diffusion magnetic resonance (DTI)) and functional (electroencephalography (EEG) or functional magnetic resonance $(\mathrm{fMRI}))$ methods ${ }^{331}$. Among the latter, temporal resolution of the EEG allows assessing the fast modulation of functional brain connectivity related to cognitive performance.

Neural networks are contributed by a large number of factors, some susceptible of a genetic regulation. Among them, the excitatory/inhibitory balance may play a relevant role ${ }^{332}$. On the other hand, abnormalities in SW properties of the brain networks have been found in the psychotic disorders ${ }^{333-335}$, for which a high heritability is established 46. Thus, to identify genetic contributions to network organization one possible strategy is to assess the influence of gene-related factors: i) contributing to neurotransmission, neurodevelopment and excitatory/inhibitory balance, and ii) implicated in the risk for psychosis.

Genetic variation for Neuregulin 1 (NRG1) seems to comply with both conditions. NRG1 gene codifies a pleiotropic growth factor protein with at least fifteen different isoforms. All of them have an epidermal growth factor domain (EGF), which preferentially activates tyrosine kinase receptor ErbB4. Those receptors were principally located in the postsynaptic density of parvalbumin-positive interneurons ${ }^{162,336}$. NRG1 protein is related to central nervous system development, plasticity, myelination ${ }^{337}$, migration ${ }^{338}$ and inhibitory/excitatory balance ${ }^{339,340}$. This protein and its interaction with ErbB4 could regulate glutamatergic transmission by modulating the activity of $\mathrm{N}$ methyl-d-aspartate (NMDA) ${ }^{339,341,342}$ and $\alpha$-amino-3-hydroxy-5-methyl-4-isoxazole propionic acid (AMPA) ${ }^{340}$ receptors in the excitatory synapsis. In addition, NRG1 has a very relevant role in GABAergic interneurons, one of the main cells contributing to oscillatory activity ${ }^{343,344}$, through the regulation of the differentiation, migration and activity of this type of neurons ${ }^{338}$. Although genetic variation at NRG1 loci has not been consistently associated with psychotic disorders risk in genome-wide studies, other data support that it may play a role in the risk for schizophrenia $339,345,346$. 
Therefore, it seems of interest to assess the relation between NRG1 genetic variation relevant for psychosis and functional brain network organization. For this purpose, three NRG1 SNPs were selected due to its relation with major psychoses: rs6994992, whose T allele has been related to the risk of psychosis ${ }^{347,348}$, cognitive deficits and lower brain activity in schizophrenia 343,348; and. rs6468119 and rs7005606, whose T alleles have been associated with the risk of bipolar disorder ${ }^{157,349}$. Hence, the aim of the study was to assess the possible relation between genetic variations at $N R G 1$ gene and $S W n$ modulation during a cognitive task. The assessment of the association of brain network modulation with genetic variation in healthy population can be a first step to understand how genetic background influences the disconnectivity in clinical psychosis.

\subsection{Methods}

\subsubsection{Participants: demographic and clinical assessment}

We recruited 144 healthy controls through newspaper announcements, all of them from Caucasian race. A semi-structured interview prior to the study was used to discard current or past psychiatric diagnoses or treatments. Exclusion criteria were: (i) comorbid axis-1 diagnosis; (ii) family antecedents of psychosis; (iii) psychoactive treatment; (iv) substance abuse; ( $v$ ) history of head trauma or any disease affecting the central nervous system; and (vi) intelligence quotient (IQ) below 70. Socio-demographic data are shown in table 6.1.

\subsubsection{Electrophysiological recordings}

EEG recordings were acquired during the performance of an auditory odd-ball task. The task consisted on random series of 600 stimulus (duration $50 \mathrm{~ms}$, rise and fall time $5 \mathrm{~ms}$, intensity $90 \mathrm{~dB}$ ) composed by three different tones with different probabilities: (i) S1, $2000 \mathrm{~Hz}$ standard tone, probability 0.60; (ii) S2, $1000 \mathrm{~Hz}$ distractor tone, probability 0.20 ; and (iii) S3, $500 \mathrm{~Hz}$ target tone, probability 0.20 . The subjects were asked to press a buttom when they listened the target tone. During the recording, they remained seated, relaxed and with their eyes closed. Only attended target tones were considered for further analysis. 
EEG activity was recorded using a Brain Vision electroencephalographic system (Brain Products, Germany), with 17 electrodes (Fp1, Fp2, F3, F4, F7, F8, C3, C4, P3, P4, O1, O2, T5, T6, Fz, Pz and Cz) placed following the specifications of the 10-20 international system (Electro-Cap International, Inc.; Eaton, OH, USA). Electrooculogram was also recorded to detect eye movements. Impedance was kept under $5 \mathrm{k} \Omega$ during EEG acquisition. The sampling rate was $500 \mathrm{~Hz}$.

\subsubsection{Signal processing}

\subsubsection{EEG Processing}

Each EEG recording was initially re-referenced over $\mathrm{Cz}$ electrode to minimize the effect of microsaccadic artifacts ${ }^{350}$. Then, EEG signals were bandpass filtered between 1 and $70 \mathrm{~Hz}$. In addition, a $50 \mathrm{~Hz}$ notch filter was used to remove the power line artifact. Independent component analysis (ICA) was applied to visually discard components related to ocular and muscular artifacts. A segmentation into $1 \mathrm{~s}$-length trials, ranging from -300 ms before stimulus onset to $700 \mathrm{~ms}$ after stimulus onset, was performed. Finally, an adaptive thresholding method was used to discard those epochs whose amplitude exceeded the threshold ${ }^{351}$.

\subsubsection{Continuous wavelet transform}

Continuous wavelet transform (CWT) was used to estimate time-frequency maps, since it is a suitable technique to analyze non-stationary recordings ${ }^{352}$. In this study, we used two different time windows: (i) the baseline window, $[-300,0]$ ms previous to the stimulus onset; and (ii) the response window, $[150,450]$ ms post-stimulus ${ }^{351}$. In order to avoid CWT edge effects in short-time recordings, a cone of influence (COI) was defined for the two windows under study ${ }^{353}$. It is noteworthy that evoked response is included in the response window.

Five frequency bands were considered for subsequent analyses. They were defined according to the conventional EEG frequency bands: theta $(\theta, 4-8 \mathrm{~Hz})$, alpha $(\alpha, 8-13$ $\mathrm{Hz})$, beta-1 $(\beta 1,13-19 \mathrm{~Hz})$, beta-2 $(\beta 2,19-30 \mathrm{~Hz})$, and gamma $(\gamma, 30-70 \mathrm{~Hz})$. Delta frequency band was not analyzed, since it is associated with a wavelet duration of hundreds of milliseconds ${ }^{351}$. 


\subsubsection{Mean Squared Coherence Complex Network Theory}

Coherence measure assesses the functional interaction between couple of signals from a specific brain region ${ }^{354}$. In this study, mean squared coherence (MSC) was used to quantify the spectral content between couples of EEG channels. Therefore, MSC between two signals is the cross function of the normalized power spectral density divided by the normalized power spectral density of the two signals separately ${ }^{354}$ :

$$
M S C_{X Y}(t, f)=\frac{\left|W S_{X Y}(t, f)^{2}\right|}{W S_{X X}(t, f) \cdot W S_{Y Y}(t, f)},(1)
$$

where $W S_{X Y}$ is the cross-spectral density of two signals ( $X$ and $\left.Y\right)$ from the wavelet scalogram (which summarizes the distribution of the signal energy in the time-frequency plane), and $W S_{X X}$ and $W S_{Y Y}$ the auto-spectral density functions from the wavelet scalogram. Finally, MSC values were averaged in each of the spectral bands under study.

\subsubsection{Complex Network Theory}

To model a system as a graph, nodes may represent the dynamical units and their links stand for the interactions between them ${ }^{355}$. We have used the SWn parameter, since it summarizes two different properties of a network: integration and segregation. First, cluster coefficient (CIC, a segregation measure) is computed for each node of the network and each frequency band:

$$
C l C_{i}^{b}=\frac{\sum_{k=i} \sum_{\substack{l \neq i \\ l \neq k}} w_{i k}^{b} w_{i l}^{b} w_{i l}^{b}}{\sum_{k=i} \sum_{\substack{l \neq i \\ l \neq k}} w_{i k}^{b} w_{i l}^{b}}, b \in\{\theta, \alpha, \beta 1, \beta 2, \gamma\}(2)
$$

where $\mathrm{b}$ represent the frequency band under study. And $w_{i j}^{b}$ the weight between two nodes provided by the MSC measure. Second, averaged path length (PL, an integration measure) was computed for each node following the following equation:

$$
P L_{i}^{b}=\frac{\sum_{j \neq i} d_{i j}^{b}}{N-1}, b \in\{\theta, \alpha, \beta 1, \beta 2, \gamma\}
$$

where $\mathrm{N}$ is the total number of nodes of the network (17 in this case) and $d_{i j}^{b}$ is the minimum distance between nodes $\mathrm{i}$ and $\mathrm{j}$. Then, $\mathrm{ClC}$ and $\mathrm{PL}$ values were averaged over all nodes in order to obtain global parameters of the network. Finally, in order to obtain 
measures that are independent of the network size, CLC and PL were normalized dividing by $<\mathrm{CLC}_{\text {surrogate }}>$ and $<\mathrm{PL}_{\text {surrogate }}>$ :

$$
\begin{gathered}
P L_{n}^{b}=\frac{P L}{\left\langle P L_{\text {surrogate }}\right\rangle},(4) \\
C l C_{n}^{b}=\frac{C l C}{\left\langle P L_{\text {surrogate }}\right\rangle},(5)
\end{gathered}
$$

where $<\mathrm{CLC}_{\text {surrogate }}>$ and $<\mathrm{PL}_{\text {surrogate }}>$ denote weighted clustering coefficient and path length averaged over an ensemble of 50 surrogate random networks that were derived from the original ${ }^{356}$. Therefore, SW was independent of the network size ${ }^{357}$ :

$$
S W n^{b}=\frac{C l C_{n}^{b}}{P L_{n}^{b}}(6)
$$

\subsubsection{Parameter baseline correction}

The baseline correction process is used to achieve a stimulus-independent characterization ${ }^{351}$. Once the spectral parameters were computed for each temporal 1 s-length trial, they were decomposed into the baseline and the response window ${ }^{358}$ Firstly, the spectral analysis provides a value for each temporal window. Secondly, the values of the previous parameters in the $[-300,0] \mathrm{ms}$ interval were averaged to obtain a baseline parameter mean. Then, the baseline correction was carried out using a simple subtraction. For that purpose, the SWn during the baseline window for each frequency band under study $\left(S W n_{B L}^{b}\right)$ is subtracted from the response value $\left(S W n_{R}^{b}\right)$ for each participant (mean of the values in the $[150,450]$ ms interval) obtaining the corrected $S W \mathrm{n}\left(S W n_{M}^{b}\right)$. It should be noted that negative values indicate a parameter decrease in the response window, while positive values represent an increase from baseline to response window.

$$
S W n_{M}^{b}=<S W n_{R}^{b}-S W n_{B L}^{b}>(7)
$$

where $<>$ denotes the average across trials.

\subsubsection{Genetic analyses}

We obtained from each individual approximately $10 \mathrm{ml}$ of venous blood using $\mathrm{K} 3$ EDTA (ethylenediaminetetraacetic acid) tubes. The samples were centrifuged with 
Ficoll-Paque following the Trizol protocol (Invitrogen, Carlsbad, CA, USA) to obtain genomic DNA from white cells. The genotyping was carried out via TaqMan assays through real-time polymerase chain reaction (PCR) with custom-made probes and primers (Applied Biosystems, Foster City, CA). The final volume of reaction was $10 \mu \mathrm{l}$ and the amplification program protocol used the following temperatures for 40 cycles: 94 ㅇ $\mathrm{C}$ (denaturing), 60ㄷ (annealing) and $72{ }^{\circ} \mathrm{C}$ (extension).

The polymorphism distribution results were evaluated with chi-squared tests to analyze Hardy-Weinberg equilibrium. SNPs rs6994992 and rs7005606 showed HardyWeinberg equilibrium ( $p=0.8248$ and $p=0.1312$ ). In contrast, SNP rs6468119 does not fit to Hardy-Weinberg proportions ( $p=0.0387)$, which could be contributed by the smaller genotyping success rate in this polymorphism (75.94\%).

\subsubsection{Statistics}

For each SNP, cases were dichotomized into non-risk allele carriers and risk alleles carriers. The distribution of SWn values was evaluated with one-sample KolmogorovSmirnov tests.

Demographic data were compared with Chi Square test or Student's $t$-test when appropriated.

The significance of $S W n$ modulation on each frequency band (or $S W n_{M}^{b}$ ) between carriers and non-carriers was assessed in each band of the EEG (except for delta) using Student's $t$-test. Moreover, to assess if possible differences in SWn modulation were contributed by the baseline window, $S W n_{B L}^{b}$, values were compared between risk and non-risk allele carriers.

\subsection{Results}

\subsubsection{Socio-demographic data}

There were no significant differences between risk and non-risk allele carriers for any SNP in age, sex distribution and school years (table 6.1). 


\subsubsection{NRG1 SNP and SW}

SWn values for each subgroup of risk allele carriers and non-risk allele carriers were normally distributed according to results of Kolmogorov-Smirnov tests (range of values: $Z$ values from 0.309 to 1.075 and $p$ values from 0.198 to 1$)$.

There were significant differences in SWn modulation between rs6468119 carriers and non-carriers in alpha and beta1 frequency bands $\left(S W n_{M}^{\alpha} .: \mathrm{t}=-2.06, \mathrm{df}=106, p=0.042\right.$; $S W n_{M}^{\beta 1}: \mathrm{t}=-2.390, \mathrm{df}=106 ; p=0.019$ ) (table 6.1). Non-risk allele carriers (CC; $n=28$ ) showed a significant decrease in $S W n_{M}^{\alpha}$ and $S W n_{M}^{\beta 1}$, while risk allele carriers (CT/TT; $n=85$ ) showed a slight $S W n$ increase in these bands (table 6.1 and figure. 6.1). $S W n_{B L}^{b}$ did not show significant differences between groups in any frequency band (table 6.2).

We also found a significant difference in theta $S W n\left(S W n_{M}^{\theta}\right)$ between rs6994992 risk and non-risk allele carriers. Both risk allele carriers (CT/TT; $n=120)$ and non-carriers (CC; $n=24$ ) showed a positive modulation of $S W n$ in this band, significantly larger in risk allele carriers ( $\mathrm{t}=-2.02, \mathrm{df}=142, p=0.046$ ) (table 6.1 and figure. 6.1). For this allele and band, $S W n$ values were significantly different at baseline in theta band $\left(S W n_{B L}^{\theta}\right)$ between risk allele carriers and non-carriers. $S W n_{B L}^{\theta}$ was significantly smaller in risk allele carriers (table 6.2).

Finally, a significant difference in SWn modulation was observed between rs7005606 risk and non-risk allele carriers in theta $\left(S W n_{M}^{\theta}\right)$ and beta-1 $\left(S W n_{B L}^{\theta}\right)$ bands. Risk allele carriers (GT/TT; $n=111$ ) showed a significantly larger positive modulation $S W n_{B L}^{\theta}$ (i.e., a larger increase from baseline to response window) than non-carriers (GG; $n=27)$ ( $t=-$ 1.99, $\mathrm{df}=136, p=0.048$ ) (table 6.1 and figure. 6.1). 
Table 6.1. Socio-demographic data and $S W n_{M}^{b}$ values for the cohort of subjects enrolled in the study. Data was divided into two groups, which were defined depending on the presence of NRG1 risk polymorphisms for psychoses. SWn values were calculated as the difference between SWn at response and baseline windows ( $\left.S W n_{R}^{b}-S W n_{B L}^{b}\right)$. Positive values represent a SW increase at response. Statistically significant differences between risk and no-risk allele carriers are marked with an asterisk: *. $p<0.05$.

\begin{tabular}{|c|c|c|}
\hline & $\begin{array}{c}\text { Non-risk allele carriers } \\
\text { Mean (SD) }\end{array}$ & $\begin{array}{c}\text { Risk allele carriers } \\
\text { Mean (SD) }\end{array}$ \\
\hline \multicolumn{3}{|l|}{ rs6468119 } \\
\hline$N$ & 28 & 85 \\
\hline Age (years) & $26.64(8.57)$ & $27.24(8.59)$ \\
\hline Gender (M:F) & $12: 16$ & $35: 50: 00$ \\
\hline Education (years) & $15.89(1.57)$ & $16.89(1.85)$ \\
\hline SWn-theta & $0.25(0.57)$ & $0.42(0.5)$ \\
\hline SWn-alpha & $-0.14(0.36)^{*}$ & $0.05(0.43)$ \\
\hline SWn-beta1 & $-0.12(0.28)^{*}$ & $0.03(0.29)$ \\
\hline SWn-beta2 & $-0.04(0.13)$ & $0.01(0.17)$ \\
\hline SWn-gamma & $-0.01(0.08)$ & $0.01(0.06)$ \\
\hline \multicolumn{3}{|l|}{ rs6994992 } \\
\hline$N$ & 24 & 120 \\
\hline Age (years) & 28.29 (10.9) & $27.41(8.87)$ \\
\hline Gender (M:F) & $14: 10$ & 48:72 \\
\hline Education (years) & 16.69 (1.89) & $16.07(2.32)$ \\
\hline$S W n$-theta & $0.14(0.56)^{*}$ & $0.36(0.48)$ \\
\hline SWn-alpha & $-0.13(0.39)$ & $-0.02(0.41)$ \\
\hline SWn-beta1 & $-0.06(0.33)$ & $-0.03(0.31)$ \\
\hline SWn-beta2 & $-0.03(0.14)$ & $-0.02(0.16)$ \\
\hline sWn-gamma & $-0.003(0.07)$ & $-0.003(0.06)$ \\
\hline \multicolumn{3}{|l|}{ rs7005606 } \\
\hline$N$ & 27 & 111 \\
\hline Age (years) & $26.44(10.16)$ & $27.96(9.11)$ \\
\hline Gender (M:F) & $12: 15$ & $43: 68$ \\
\hline Education (years) & $16.16(1.64)$ & $16.27(2.44)$ \\
\hline SWn-theta & $0.15(0.48)^{*}$ & $0.36(0.48)$ \\
\hline$s W n$-alpha & $-0.18(0.34)$ & $-0.02(0.41)$ \\
\hline$S W n$-beta1 & $-0.14(0.31)^{*}$ & $-0.01(0.3)$ \\
\hline SWn-beta2 & $-0.06(0.14)$ & $-0.01(0.16)$ \\
\hline SWn-gamma & $-0.02(0.07)$ & $0.002(0.06)$ \\
\hline
\end{tabular}


Table 6.2. $S W n_{B L}^{\theta}$ values in baseline window for NRG1 SNPs. The statistically significant differences between risk alleles carriers and non-risk allele carriers are marked with asterisks: ${ }^{*} p<0.05 ;{ }^{* *} p<0.01 ; * * * p<0.001$

\begin{tabular}{l|c|c}
\hline & $\begin{array}{c}\text { Non-risk allele carriers } \\
\text { Mean (SD) }\end{array}$ & $\begin{array}{c}\text { Risk allele carriers } \\
\text { Mean (SD) }\end{array}$ \\
\hline rs6468119 & $1.92(0.40)$ & $1.91(0.57)$ \\
\hline Theta & $2.68(0.99)$ & $2.73(0.97)$ \\
Alpha & $2.03(0.52)$ & $2.02(0.53)$ \\
Beta1 & $1.49(0.33)$ & $1.46(0.31)$ \\
Beta2 & $1.09(0.07)$ & $1.09(0.08)$ \\
Gamma & & $1.84(0.49)$ \\
\hline rs6994992 & $2.26(0.70)^{* *}$ & $2.58(1.01)$ \\
\hline Theta & $3.35(1.16)^{* * *}$ & $1.98(0.54)$ \\
Alpha & $2.29(0.62)^{*}$ & $1.44(0.30)$ \\
Beta1 & $1.53(0.32)$ & $1.09(0.08)$ \\
Beta2 & $1.10(0.08)$ & $1.09(0.08)$ \\
Gamma & $1.12(0.07)$ & $1.90(0.54)$ \\
\hline rs7005606 & $1.92(0.48)$ & $2.70(1.06)$ \\
\hline Theta & $2.66(1.15)$ & $2.03(0.56)$ \\
Alpha & $1.99(0.58)$ & $1.45(0.31)$ \\
Beta1 & $1.43(0.32)$ & \\
Beta2 & & \\
\hline & & \\
\hline
\end{tabular}

Moreover, risk allele carriers showed a significantly lower negative modulation in beta- 1 band (less decrease in $S W n_{B L}^{\theta}$ from baseline to response) ( $\mathrm{t}=-2.06, \mathrm{df}=136$, $p=0.042$ ) (table 6.1 and figure. 6.1). There were no significant differences at $S W n_{B L}^{b}$ for this allele. 


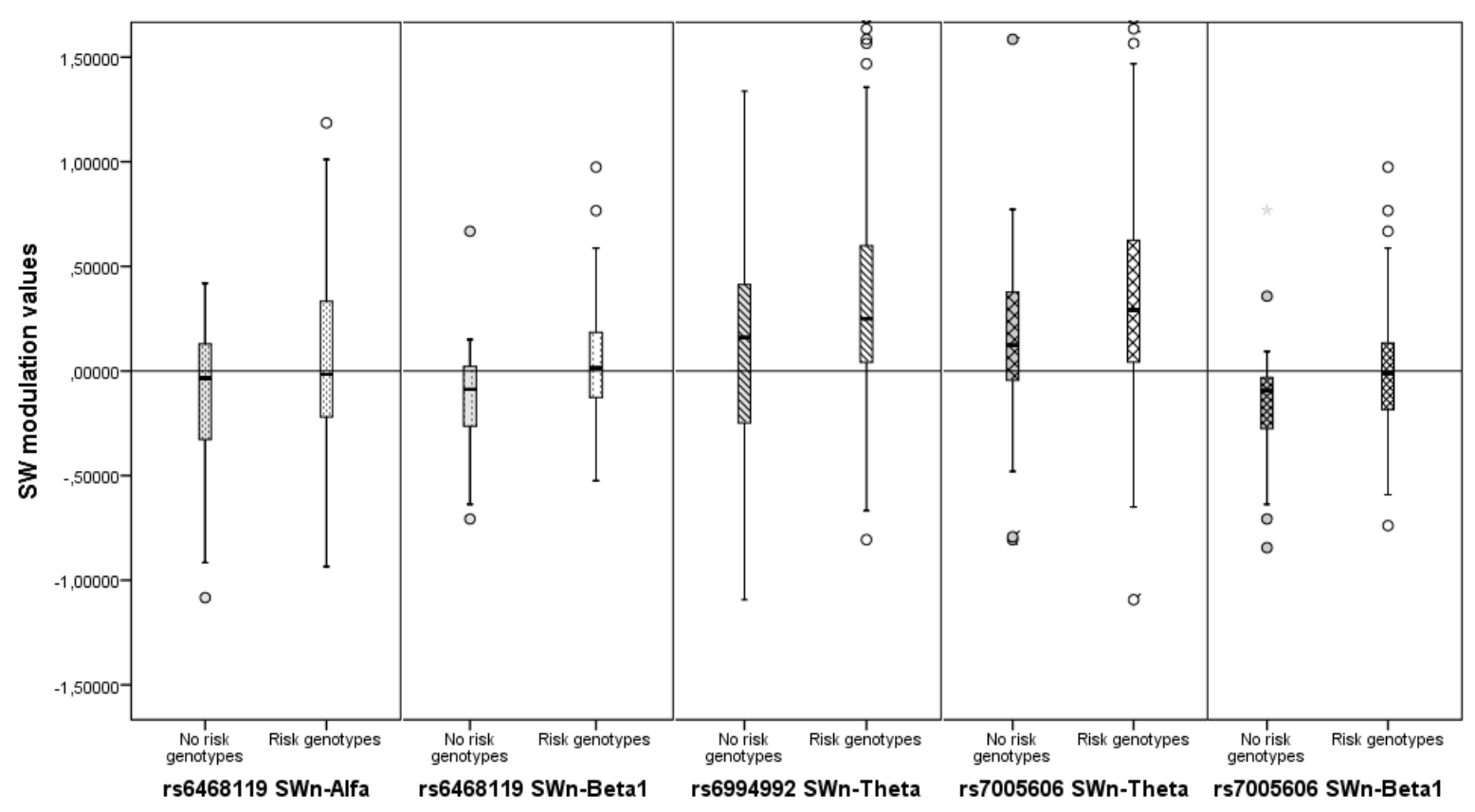

Figure 6.1. Distribution of significant $S W n_{M}^{b}$ modulation values $\left(S W n_{R}^{b}-S W n_{B L}^{b}\right)$ in non-risk allele carriers and risk allele carriers.

\subsection{Discussion}

We found a significant association between genetic variation at NRG1 and the modulation of SW properties of the functional cortical network during the performance of an odd-ball task in healthy subjects. As far as we know, no previous studies investigated the effect of NRG1 polymorphisms on functional brain network modulation. SWn modulation in the theta band, $S W n_{M}^{\theta}$ was associated with two NRG1 SNPs: rs6994992 and rs7005606. In both cases risk allele carriers presented larger $S W n_{M}^{\theta}$ (more positive $S W n$ changes) than non-risk allele carriers. Theta oscillations are related to long-range synchronization in brain networks ${ }^{359,360}$. According to our data, the risk allele may be associated with a larger modulation of long-range connectivity in response to a task. Such a larger modulation was associated with lower $S W n_{B L}^{\theta}$ values in risk allele carriers. Therefore, risk allele carriers with smaller SWn values in the baseline window might have to increase the $S W n$ in this band to a larger extent than non-carriers during task performance. 
NRG1 rs6994992 is located at the promotor region of the gene, inside the HAPice haplotype ${ }^{342}$, being the only functional HAPice polymorphism and related to cognitive alterations ${ }^{346}$. Rs6994992 has also been related to white matter integrity in healthy subjects $^{345}$. Thus, variation at this locus might contribute to the structural substrate of long-range connectivity ${ }^{361}$. However, previous studies showed a reduced fractional anisotropy in anterior limb of internal capsule and anterior thalamic radiation in risk allele carriers ${ }^{345,362}$. Therefore, the role of $N R G 1$ variation on white matter integrity may not justify the association observed in the present study with fast regulation of cortical network in the theta band.

Our findings also support an association between rs6468119 and rs7005606 and SWn modulation in beta 1 band, risk allele carriers showing smaller SW modulation. The same result was observed regarding $S W n$ modulation in alpha band respect rs6468119. Alpha and beta bands may also play a role in long-range synchronization ${ }^{363,364}$. Both SNPs are non-coding polymorphisms 157,349 . Their specific effects are unknown, but they could be related to the regulation of NRG1 transcription ${ }^{339,365}$. Either increase or decrease of the NRG1 is related to changes in the inhibitory/excitatory balance, supporting its possible contribution to connectivity supported by cerebral oscillations: an increase of NRG1ErbB4 signaling is related to a decrease of NMDAr activation and currents ${ }^{366,367}$, mainly through the inhibition of Src kinases pathway ${ }^{62}$. Also, hypofunction of NRG1-ErbB4 might cause abnormal glutamatergic transmission through increasing NR2B phosphorylation ${ }^{368}$. Furthermore, the NRG1 increase is associated with an impairment of GABA release ${ }^{369}$ and NRG1 increase or decrease is respectively related to promotion or reduction of miniature excitatory postsynaptic currents in GABAergic interneurons 370 that impair pyramidal neurons synchronization ${ }^{371}$.

We a priori selected SNPS on the basis of the likely risk conferred by one of their respective alleles for major psychosis, in which a decreased $S W \mathrm{n}$ has been reported. However, contrary to our expectations, risk alleles in healthy subjects were rather associated with a larger SWn modulation during a cognitive task. As previously stated, this may be in part justified by smaller $S W n$ values at rest, but it also seems compatible with the possibility that other genetic and non-genetic factors may have a larger and complex influence in the network organization in schizophrenia patients. 
Among the study limitations, larger samples are needed to adequately detect influences of genotype on brain networks modulation. Improved genotyping success is needed before our rs6468119 genotyping results could be extrapolated to the general population. Moreover, we measured individually the effect of every SNP, but the effect of genes in brain networks is more probably epistatic or additive ${ }^{361}$.

As a conclusion, our data are consistent with an effect of NRG1 on functional connectivity ${ }^{372}$, perhaps through variation in expression of NRG1-ErbB4 pathway, which could contribute to GABAergic and glutamatergic imbalance. Since our results are significant in theta, alpha and beta-1 frequencies bands, which may support transitory, task-related coupling between distant areas of the brain ${ }^{373}$, we could infer that genetic variation at NRG1 may influence on brain networks mostly in the modulation of longdistance connectivity in the brain 


\section{Chapter 7}

\section{Analysis of KCNH2 and CACNA1C}

\section{schizophrenia risk genes on EEG functional}

\section{network modulation during an auditory}

\section{odd-ball task}

\section{Submitted}

Authors: Alba Lubeiro ${ }^{a^{*} *}$ \& Mar Fatjó-Vilas ${ }^{b, c, d^{*} *}$, Maria Guardiola ${ }^{b, c, d}$, Carmen Almodóvar ${ }^{b}$, Javier Gomez-Pilare, Benjamin Cea-Cañas ${ }^{f}$, Jesús Poza ${ }^{\mathrm{e}, \mathrm{g}, \mathrm{h}}$, Aitor Palominoi, Marta Gómez-García j, Jone Zugastik ${ }^{k}$ Vicente Molina ${ }^{a, d, g, j}$

a. Psychiatry Department, School of Medicine, University of Valladolid, Valladolid, Spain.

b. FIDMAG Germanes Hospitalàries Research Foundation, Barcelona, Spain.

c. Evolutive biology, ecology and environment Department University of Barcelona, Spain.

d. CIBERSAM: Biomedical Research Network: Mental Health; Instituto de Salud Carlos III).

e. Biomedical Engineering Group, Department TSCIT, University of Valladolid, Spain.

f. Neurophysiology service, University Hospital of Valladolid, Valladolid, Spain.

g. Neurosciences Institute of Castilla y León (INCYL), University of Salamanca, Spain.

h. IMUVA, Mathematics Research Institute, University of Valladolid, Spain.

i. Neurotek, Neurosciences Department, University of Basque Country, Vizcaya, Spain.

j. Psychiatry service, University Hospital of Valladolid, Valladolid, Spain.

k. Psychiatry Department, University Hospital of Álava, Álava, Spain.

* Co-corresponding authors.

* Authors AL and MF-V contributed equally to this work. 


\section{Abstract}

A deficit in task-related functional connectivity modulation of the electroencephalographic (EEG) network has been previously described in schizophrenia. The use of measures of neuronal connectivity as an intermediate phenotype may allow identifying genetic factors involved in these deficits and therefore establishing underlying pathophysiological mechanisms. Genes involved in neuronal excitability and electric transmission and previously associated with the risk for schizophrenia may be adequate candidates in relation to functional connectivity alterations in schizophrenia.

Two polymorphisms of voltage-gated ion channels CACNA1C ( $r$ 1006737) and KCNH2 (rs3800779) were genotyped in 101 controls and 50 patients with schizophrenia. Small worldness index (SWn) was calculated from electroencephalography (EEG) signals recorded during an odd-ball task in two different temporal windows (pre-stimulus and response). Modulation was defined as the difference in SWn values between both windows. Genetic effect on SWn in the pre-stimulus window and in modulation was evaluated.

The CACNA1C genotype was not associated with SW properties. KCNH2 was significantly associated with SWn modulation. Patients carrying the risk allele (A) showed a negative SWn modulation while patients not carrying the risk allele and controls showed a positive SWn modulation.

Our data suggest that $K C N H 2$ genotype contributes to the modulation efficiency of brain electrophysiological activity during a cognitive task in schizophrenia patients. Further studies with larger samples are required to confirm these results and to extend the knowledge of the implication of $K C N H 2$ and $C A C N A 1 C$ in schizophrenia brain functional connectivity phenotypes.

\subsection{Introduction}

Schizophrenia underpinnings remain largely unknown, which may relate to its complexity and heterogeneity. In this regard, endophenotypes reducing phenotypic complexity by means of intermediate phenotypes can contribute to shedding light on its 
neurobiological mechanisms ${ }^{374,375}$. Electroencephalography (EEG) is an excellent tool for studying neurophysiological intermediate phenotypes in schizophrenia ${ }^{376}$. Oscillations in neuronal activity are known to play a crucial role in information processing and cortical communication ${ }^{144}$ and are, therefore, considered not just biomarkers of, but essential components in neural communication and computation. Accordingly, data collected with EEG reflect synchronously firing neuronal assemblies and allow the study of functional connectivity during cognitive processes with high temporal resolution. Changes in brain functional connectivity have been suggested in the pathophysiology of schizophrenia ${ }^{115-117,377}$ and, to a lesser extent, have also been detected in first degree healthy relatives of schizophrenia patients ${ }^{378}$.

The application of graph theory to EEG activity measures may help in refining the characterization of connectivity patterns in patients with schizophrenia. Brains minimize energy expenditure by combining highly clustered short-range local connections and maximizing integration of information between distant brain areas with a few longrange connections ${ }^{379,380}$. Such networks are named "small-world" (SW) networks and the Small-Worldeness (SWn) index reflect the closeness of a given network to that ideal structure. Higher SWn index relates to better information transmission capacity in a functional network ${ }^{332}$. SWn results from dividing the global clustering coefficient (CLC) by characteristic path length (PL). CLC quantifies the connections of a node with their neighbour nodes and is interpreted as a measure of brain segregation. The characteristic $\mathrm{PL}$ is the average of all the shortest paths between each pair of nodes in the network and it reflects integration between distant nodes. One advantage of functional networks studies is the possibility of assessing its modulation with cognitive activity (i.e. the degree of connectivity changes associated with the cognitive demand). In one such study during a P300 paradigm, the functional network of schizophrenia patients was characterized by smaller SWn task-related modulation than healthy controls ${ }^{117}$.

Since schizophrenia is a genetically complex disorder with a heritability around $80 \%$ 49 , the use of intermediate phenotypes may also allow identifying disease-related genetic signatures ${ }^{374,381}$. In this respect, the fact that neural oscillations are highly heritable traits in humans ${ }^{382,383}$, supports the putative role of EEG as an intermediate phenotype in the pathway from genes to behaviour ${ }^{384}$. As regards to resting-state EEG 
functional connectivity, previous studies have also reported an important heritability across various frequency bands: $23-89 \%$ for CLC and 37-62\% for PL 330,385. Despite evidence about genetic influences on EEG parameters, current understanding of specific genes or variants involved in electrophysiological phenotypes remains limited. On the one hand, recent genome-wide association studies (GWAS) have identified several candidate genes for schizophrenia, most of them converging on molecular complexes related to neuronal excitability and therefore also to functional connectivity processes. Some of those genes are related to voltage-dependent calcium channels, post-synaptic excitatory synapses components and gene expression regulation factors ${ }^{48,386}$. On the other hand, EEG components have been significantly correlated with genes and gene clusters enriched for biological properties affecting neural circuitry, involved in the neuronal activity and/or neurodevelopment ${ }^{387-389}$. Among these genetic candidates affecting neuronal excitability and electric transmission, we selected two genes in two voltage-gated ion channels (CACNA1C and $K C N H 2$ ) as candidates to be studied in relation to functional connectivity alterations in schizophrenia.

CACNA1C codifies for a pore protein $(\alpha 1 C)$ of $L$ type voltage-gated calcium channels Cav1.2, distributed throughout the brain 390 and primary mediators of neuron membrane polarity changes as well as of gene expression regulation ${ }^{391,392}$. In vitro and conditional knockout mouse models have shown that a decreased activity of $\mathrm{Ca}_{\mathbf{v}} 1.2$ channels is related to an increased excitatory/inhibitory (E/I) ratio, to an altered synaptic protein composition and also to anxiety-like behavior and social behavior abnormalities ${ }^{393,394}$. The critical role of $\mathrm{Ca}_{v} 1.2$ in schizophrenia is highlighted by human genetic and imaging studies. From GWAS studies several SNPs in linkage disequilibrium at CACNA1C gene have been associated with neuropsychiatric disorders 48,386,395,396. The polymorphism rs1006737 at CACNA1C and the risk effect of the A allele has been replicated in different association studies and meta-analyses ${ }^{397-403}$. In addition, the risk allele has also been related to: i) larger total and/or regional GM volumes both in patients with schizophrenia ${ }^{404}$ and healthy subjects ${ }^{63,405-407}$, ii) altered functional activity ${ }^{401,408,409}$ and connectivity ${ }^{405,408}$ during cognitive tasks ${ }^{410,411}$. Accordingly, it is hypothesized that this polymorphism is related to variability in calcium currents and CACNA1C expression which in turn might affect transcriptional regulation of other 
genes involved in neuronal signalling and excitability $401,412,413$.

$K C N H 2$ gene encodes the pore-forming subunit of an ether-a-go-go (hERG)-family potassium channel, which is expressed in excitatory cells and modulates neuronal firing patterns ${ }^{414,415}$. A meta-analysis has associated it with schizophrenia ${ }^{416}$, although not the most recent GWAS studies ${ }^{386,396}$. Specifically, the A allele of $K C N H 2$ rs3800779 polymorphism has been related to lower IQ and altered speed of cognitive processing and memory in both patients and healthy controls ${ }^{416,417}$. Furthermore, it has also been associated with decreased hippocampal volume and increased hippocampal and prefrontal activation in healthy controls ${ }^{416}$. It is noteworthy that the risk allele $(A)$ seems to be involved in an increased expression of $K C N H 2$ 3.1, a primate isoform enriched in the brain with specific electrophysiological characteristics 416 and associated with alterations in dendritic spines, impaired cognition and altered activation in the prefrontal cortex and hippocampus in a mouse model ${ }^{418}$.

Accordingly, our objective was to analyze the effect of two schizophrenia risk genes relevant to neuronal excitability and connectivity (CACNA1C and $K C N H 2$ ) on the changes in functional connectivity during the performance of a cognitive task (as measured with the EEG Small-Worldness index modulation), both in individuals with schizophrenia and healthy controls.

\subsection{Material and methods}

\subsubsection{Sample description}

We recruited 50 Spanish Caucasian patients according to DSM-V-TR criteria diagnosis of schizophrenia. Diagnoses were made or confirmed by an expert clinician (V.M.). Twenty-five of them were first-episode patients with an illness duration shorter than one year. All patients were receiving stable doses of antipsychotics. Symptoms were scored using the Positive and Negative symptoms scale (PANSS). Exclusion criteria included: medical illnesses affecting the central nervous system, substance-induced psychotic disorder, neurological conditions, history of head trauma with loss of consciousness, and $\mathrm{IQ}<70$. 
The control sample consisted of 101 Spanish Caucasian unrelated adult healthy individuals. They met the same exclusion criteria as patients as well as any current psychiatric disorder or treatment.

All participants provided written consent after full written information. The study was performed in accordance with the guidelines of the institutions involved and approved by the local ethics committee. All procedures were carried out according to the Declaration of Helsinki. Demographic and clinical variables are shown in Table 7.1.

Table 7.1. Sociodemographic and clinical information of patients and controls. Statistically significant differences were detected only in relation to patients' years of education (in bold, $p<0.01$ ).

\begin{tabular}{l|c|c}
\hline & $\begin{array}{c}\text { Controls } \\
n=101\end{array}$ & $\begin{array}{c}\text { Patients } \\
n=50\end{array}$ \\
\hline Sex (M:F) & $51: 50$ & $29: 21$ \\
Age (mean and sd) & $29.52(9.83)$ & $32.60(9.80)$ \\
Education (years) & $15.98(2.07)$ & $\mathbf{1 4 . 2 1 ( 3 . 7 7 )}$ \\
Father education (years) & $13.19(3.92)$ & $11.72(4.61)$ \\
PANSS Positive & - & $12.92(5.47)$ \\
PANSSNegative & - & $17.72(7.39)$ \\
PANSS General & - & $25.16(12.43)$ \\
PANSS Total & - & $55.34(22.51)$ \\
IIIness duration (months) & - & $80.76(107.68)$ \\
CPZ equivalents (mg) & - & $355.02(273.49)$ \\
\hline
\end{tabular}

\subsubsection{Molecular analysis}

Genomic DNA was extracted from blood using standard methods. Two SNPs were genotyped: i) rs1006737 (A/G, intron variant, chromosome position 2236129) at CACNA1C gene (12p13.33); ii) rs3800779 (A/C; intron variant, chromosome position 150974126) at $K C N H 2$ (7q36.1). Genotyping was performed using a fluorescence-based allelic discrimination procedure (Applied Biosystems Taqman 5'-exonuclease assays). Standard conditions were used. Polymerase chain reaction plates were read on $A B I$ 
PRISM 7900HT instrument with SDS v2.1 software (Applied Biosystems). The genotyping call rate for both SNPs was higher than 99\%. The accuracy of the method was tested by re-genotyping a $10 \%$ of the samples and confirming all the repeated genotypes. The two SNPs were in Hardy-Weinberg equilibrium.

Based on the risk allele and the genotypic frequency, for the statistical analyses, both patients and controls were classified as risk-allele carriers (for CACNA1C: AA+AG; for KCNH2: AA+AC) or non-carriers (for CACNA1C: GG; for KCNH2: CC) (Table 7.2).

Table 7.2. Information on SNPs included in this study. The table includes the dbSNP number, the genomic and gene position and the alleles of each SNP (GRCh38.p7). Observed genotypic and allelic frequencies are also given.

\begin{tabular}{|c|c|c|c|c|c|c|c|c|c|}
\hline GENE-SNP & Chr & $\begin{array}{c}\text { Chr } \\
\text { Position* }\end{array}$ & Position & Alleles $^{a}$ & $\mathrm{MAF}_{1000 \mathrm{G}^{\mathrm{b}}}$ & $\mathrm{MAF}_{\text {sample }}{ }^{\mathrm{c}}$ & \multicolumn{3}{|c|}{$\begin{array}{c}\text { Genotype Absolute Frequency. } \\
\text { Controls / Patients (\%) }\end{array}$} \\
\hline $\begin{array}{l}\text { CACNA1C } \\
\text { (rs1006737) }\end{array}$ & 12 & 2236129 & Intronic & $\mathrm{G} / \mathrm{A}$ & $0.302 / 0.374$ & 0.285 & $\begin{array}{c}\text { GG: } \\
0.57 / 0.50\end{array}$ & $\begin{array}{c}\text { GA: } \\
0.38 / 0.46\end{array}$ & $\begin{array}{c}\text { AA: } \\
0.05 / 0.04\end{array}$ \\
\hline $\begin{array}{l}\text { KCNH2 } \\
\text { (rs3800779) }\end{array}$ & 7 & 150974126 & Intronic & $C / A$ & $0.211 / 0.283$ & 0.305 & $\begin{array}{c}\text { CC: } \\
0.55 / 0.5\end{array}$ & $\begin{array}{c}\text { CA: } \\
0.34 / 0.36\end{array}$ & $\begin{array}{c}\text { AA: } \\
0.11 / 0.14\end{array}$ \\
\hline
\end{tabular}

${ }^{*}$ Chromosome position GRCh38 assembly.

${ }^{a}$ The less frequent allele (minor allele) is placed second.

${ }^{b}$ MAF refers to Minor Allele Frequency observed in the 1000 Genomes project across all populations / in CEU populations (Project Consortium).

'MAF observed in the current sample.

\subsubsection{EEG acquisition and processing}

EEG data were recorded in the subsample previously described during a thirteen minutes 3-tone oddball task, which consisted of 600 random sequences of target (500 $\mathrm{Hz}$-tone, probability 0.2 ), distractor (1000 Hz-tone, probability 0.2), and standard (2000 $\mathrm{Hz}$-tone, probability 0.6) tones. It was recorded using a 17-channel EEG system (BrainVision ${ }^{\circledR}$, Brain Products $\mathrm{GmbH}$ ) allocated following international 10-20 system (Fp1, Fp2, F3, F4, F7, F8, C3, C4, P3, P4, 01, O2, T5, T6, Fz, Pz and Cz). Acquisition parameters: Impedance $<5 \mathrm{k} \Omega$, sampling frecuency $=500 \mathrm{~Hz}$, referenced over $\mathrm{Cz}$. 
EEG recordings were re-referenced to the average activity of all sensors in order to minimize the effect of microsaccadic artefacts ${ }^{350}$. Data were filtered using a finite impulse response (FIR) band-pass filter (1-70 Hz, Hamming window) and a notch filter to remove the power line frequency interference $(50 \mathrm{~Hz}$, Butterworth filter).

Artefacts were rejected and continuous EEG data were segmented into 1s-length trials ranging from -300 ms before target stimulus onset to $700 \mathrm{~ms}$ after onset. Timefrequency representation of EEG was computed using continuous wavelet transform (CWT) including frequencies from 1 to $70 \mathrm{~Hz}{ }^{351,419,420}$. Thus, 1s-length evoked responses ([-300 - 700] ms) were decomposed into two windows: (i) pre-stimulus ([-300 0] ms to target stimulus onset); and (ii) response ([150 450] ms after target stimulus onset) ${ }^{351,419}$. These windows were chosen to summarize the functional modulation between prestimulus and response (assumed to summarize cognitive processing).

From each window CWT decomposition, event-related Coherence (ERC) was computed for each pair of electrodes ${ }^{351,354,419}$ and its changes in ERC between resting and active windows allowed the assessment of task-related modulation in graph parameters.

Graph parameters calculation was obtained where each electrode corresponded to nodes and ERC parameters to the weights of the links between vertex (edges). Completely filled ERC matrices were then used as adjacency matrices. From the matrices, two parameters were computed in each temporal window pre-stimulus ( $p$ ) and response: i) CLC, characterize the segregation of the network ${ }^{357}$; ii) PL, characterize the integration of the network, it is defined as the average shortest path length between all pairs of nodes in the network. CLC and $P L$ were normalized to eliminate dependencies dividing CLC and PL data by random parameters following the method proposed by ${ }^{356}$ over an ensemble of 50 surrogate network.

$$
C L C=\left(\begin{array}{l}
N \\
3
\end{array}\right) \sum_{i \in n} \sum_{j, h \in n}\left(w_{i j}^{b} w_{i h}^{b} w_{j h}^{b}\right)^{1 / 3}
$$

where $w_{x y}$ denotes the edge weight between electrodes $i$ and $j$.

$$
P L=\frac{1}{N} \sum_{i \in n} \frac{\sum_{j \in n, j \neq i} d_{i j}}{n-1},
$$


where $d_{i j}$ indicates the minimum distance (i.e. the inverse of $E R C$ ) between electrodes $i$ and $j$.

$$
S W n=\frac{C L C / C L C_{r}}{P L / P L_{r}}
$$

SWn was calculated as the ratio between segregation $(C L C)$ and integration $(P L)$. In order to achieve a stimulus-independent characterization ${ }^{351}$ and to quantify the dynamical changes during the evoked response (i.e: network modulation), SWn modulation $(S W m)$ parameter was calculated subtracting $S W$ at pre-stimulus $(S W p)$ the $S W n$ at the response window. Further description of EEG processing is detailed in ${ }^{117}$.

SWn indexes were used for the analyses as they are interpreted as good resume parameters characterizing the network efficiency and they have been shown to differ between patients and controls ${ }^{117}$.

\subsubsection{Statistical analysis}

Data were analysed using SPSS 20.0 software.

We compared age, sex distribution, years of education and parental education years between patients and controls using chi-squared or $t$-tests when appropriate.

As the SWn measures were previously compared between patients and controls in a previous report including most of the current sample ${ }^{117}$, this comparison was not included as an aim of the study. However, we did evaluate the effect of age, sex, illness duration, and treatment on patients' SWn measurements and of age and sex in healthy subjects using Pearson correlation or Student's $t$-test.

To test the association between the genetic polymorphisms and SW measures, analyses of variance (ANOVA) were performed. The effect of genotype, group (patient or control) and their interaction (genotype $x$ group) were tested on: i) SWn index at the pre-stimulus window ( $S W p$ ), and ii) $S W n$ modulation ( $S W m$ ).

To gain a better understanding of the significant detected effects, the relationship of the genotype and SWn parameters were tested separately in patients and healthy subjects with Student's $t$-tests. Also, clinical differences between patients carrying the 
risk allele and non-carriers were assessed with Student's $t$-test or U-Mann Whitney tests when appropriate.

\subsection{Results}

There were no differences in age, sex distribution or parental education between patients and controls; while, as expected, years of education were significantly lower in patients (Table 7.1). Moreover, age, sex distribution, illness duration, and treatment did not have significant effects on SW measurements, therefore, these variables were not included as covariables in the posterior analyses.

For CACNA1C, there was a significant effect of group on SWp $(F=15.098 ; p<0.001)$ and $S W m(F=5.623 ; p=0.019)$. Nevertheless, there were no significant effects of genotype or interaction for neither $S W p$ nor $S W m$. Patients carrying the risk allele showed no clinical differences in comparison to those without the risk allele (Table 7.3).

For $K C N H 2$, there was a significant effect of group on SWp (F=16.190; $p<0.001)$, with patients showing lower SWn modulation values than controls (mean (sd): patients 0.003 (0.03) and controls $0.013(0.02)$ ). Neither genotype nor interaction effects were detected on $S W p$. As regards to $S W m$, there was a significant effect of group ( $F=6.156$; $p=0.014)$, genotype $(\mathrm{F}=6.19 ; p=0.013)$ and interaction (genotype*group) ( $\mathrm{F}=6.209$; $p=0.014)$. Subsequently, a t-test revealed differences within patients, with risk-allele carriers showing negative values in SWm while non-carriers presented positive values $(\mathrm{t}=-2.84 ; \mathrm{df}=48 ; p=0.007$ ) (Figure 7.1 and 7.2 and Table 7.3). Patients carrying the risk allele did not differ in clinical variables from non-carriers (Table 7.3). Within controls, no differences between carriers and non-carriers were observed in SWp and SWm. 
Table 7.3. Sociodemographic, clinical and EEG functional connectivity variables mean and standard deviation per group and genotype. Differences between genotypic groups (risk-allele carriers vs non-carriers) within patients or controls are marked in bold $(p<0.01)$.

\begin{tabular}{|c|c|c|c|c|}
\hline $\begin{array}{l}\text { CACNA1C } \\
\text { (rs1006737) }\end{array}$ & $\begin{array}{c}\text { Controls AA/AG } \\
n=43\end{array}$ & $\begin{array}{c}\text { Controls GG } \\
\qquad n=58\end{array}$ & $\begin{array}{c}\text { Patients AA/AG } \\
n=25\end{array}$ & $\begin{array}{c}\text { Patients GG } \\
\qquad n=25\end{array}$ \\
\hline Sex (M:F) & $23: 20$ & $28: 20$ & $17: 08$ & $12: 13$ \\
\hline Age (mean and sd) & $29.98(9.98)$ & 29.19 (9.79) & $32.52(9.00)$ & $32.68(10.72)$ \\
\hline PANSS Positive & - & - & $13.11(5.79)$ & $12.74(5.29)$ \\
\hline PANSS Negative & - & - & $18.21(8.00)$ & $16.68(6.84)$ \\
\hline PANSS General & - & - & $26.11(13.87)$ & 24.21 (11.09) \\
\hline PANSSTotal & - & - & $57.37(25.11)$ & $53.32(20.05)$ \\
\hline Illness duration (months) & - & - & 73.65 (103.69) & $89.12(114.80)$ \\
\hline CPZ equivalents (mg) & - & - & $418.30(290.29)$ & $296.80(248.70)$ \\
\hline$S W n$ pre-stimulus & $1.021(0.024)$ & $1.025(0.023)$ & $0.987(0.068)$ & $1.007(0.048)$ \\
\hline$S W n$ modulation & $0.012(0.025)$ & $0.014(0.022)$ & $0.001(0.026)$ & $0.004(0.031)$ \\
\hline $\begin{array}{l}\text { KCNH2 } \\
\text { (rs3800779) }\end{array}$ & $\begin{array}{c}\text { Controls AA/AC } \\
n=45\end{array}$ & $\begin{array}{c}\text { Controls CC } \\
n=56\end{array}$ & $\begin{array}{c}\text { Patients AA/AC } \\
n=25\end{array}$ & $\begin{array}{c}\text { Patients CC } \\
n=25\end{array}$ \\
\hline Gender (M:F) & $16: 29$ & $35: 21$ & $16: 09$ & $13: 12$ \\
\hline Age (mean and sd) & $29.53(9.61)$ & 29.52 (10.09) & $32.64(10.5)$ & $32.56(9.26)$ \\
\hline Panns Positive & - & - & $13.00(5.05)$ & $12.85(5.96)$ \\
\hline Panns Negative & - & - & $20.00(8.55)$ & $15.15(5.40)$ \\
\hline Panss General & - & - & $27.11(15.20)$ & $23.4(9.34)$ \\
\hline Panss Total & - & - & $60.17(25.48)$ & $51.00(19.07)$ \\
\hline Illness duration (months) & - & - & $66.31(80.28)$ & 91.76 (125.47) \\
\hline CPZ equivalents (mg) & - & - & 375.91 (278.92) & 335.8 (272.69) \\
\hline SWn pre-stimulus & $1.03(0.018)$ & $1.018(0.027)$ & $0.993(0.067)$ & $1.000(0.051)$ \\
\hline SWn modulation* & $0.013(0.025)$ & $0.013(0.022)$ & $-0.008(0.024)$ & $0.013(0.028)$ \\
\hline
\end{tabular}




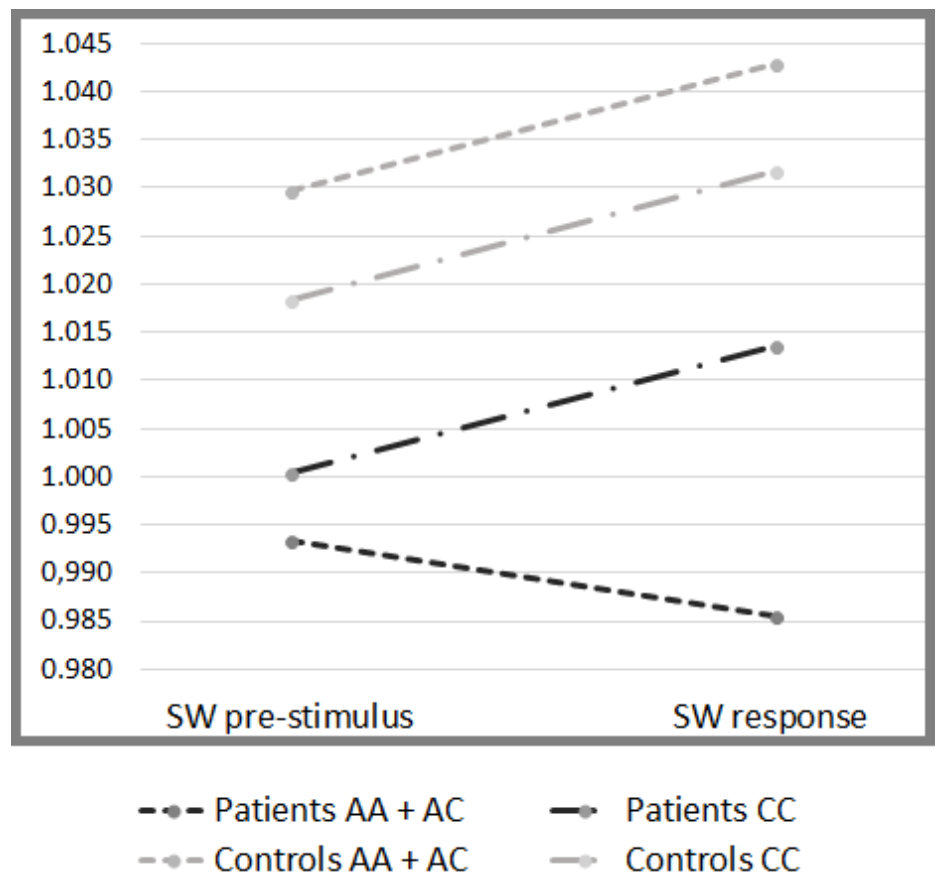

Figure 7.1. Patterns of SWn changes per group and genotype (KNCH2 rs3800779) from pre-stimulus to response windows.
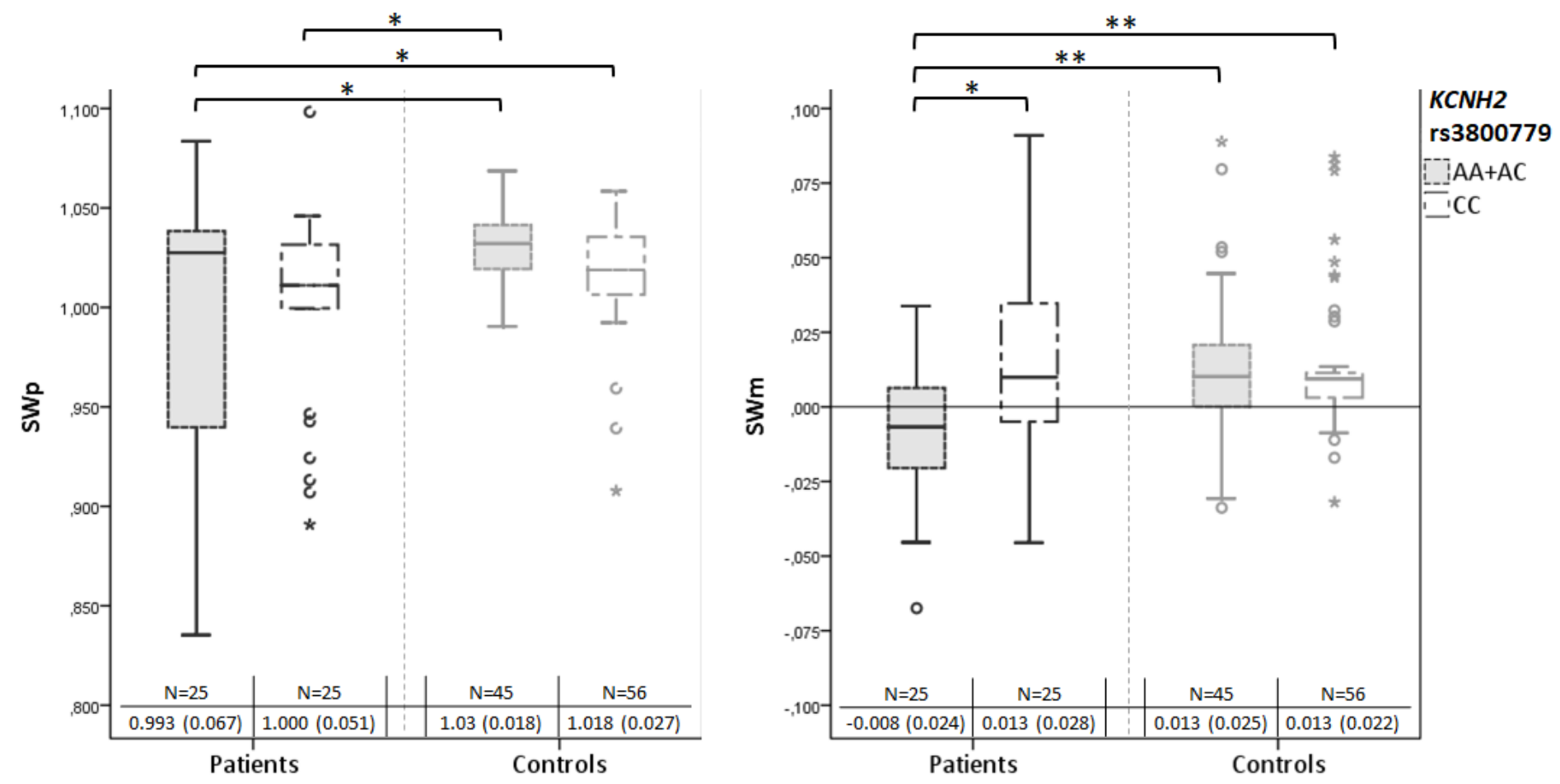

Figure 7.2. Distribution of SWn values at the pre-stimulus window (left) and modulation (right). Significant differences are marked with asterisk: ${ }^{*} p<0.05 ;{ }^{*} p<0.01$. Mean and standard deviation for each group and genotype (KCNH2 rs3800779) are in the bottom of the figure. 


\subsection{Discussion}

In the present study, we report a significant genetic effect of $K C N H 2$ on brain functional connectivity measured with EEG during an odd-ball task. However, we have not observed any effect of CACNA1C on this connectivity. In particular, patients carrying the $K C N H 2$ rs3800779 risk allele showed an opposite pattern of SWn modulation in comparison with patients not carrying the risk alleles and healthy controls. Specifically, we found that patients carrying the A allele had negative SWn modulation, which means lower SWn values at response than at pre-stimulus window, contrary to the modulation pattern in patients not carrying the risk allele and controls.

Contrary to what we expected, we did not find a significant effect of CACNA1C on brain connectivity as measured with SWn parameters (pre-stimulus or modulation). Nevertheless, the involvement of CACNA1C in SWn parameters cannot be completely excluded. First, because of the sample size and the minor allele frequency, the analyses have been conducted by comparing risk allele carriers versus non-carriers, which has not allowed testing the recessive effect of the risk allele. In this regard, a study using induced human neurons reported functional effects of the rs1006737 (i.e. increased Ca2+ current density as well as increased mRNA expression of CACNA1C) only when the risk allele was in homozygosis ${ }^{413}$. Second, as suggested by previous studies, the possible effect of CACNA1C on connectivity could depend on the effect of other close polymorphisms ${ }^{412,421}$ or on specific haplotypes ${ }^{422}$. Third, most of the evidence on the effect of CACNA1C on brain activity or connectivity comes from studies based on healthy subjects $405,408,409$. Then, there is little evidence to understand its effect in the disease context. To this respect, further studies are needed, also to better define the allelic effects as some studies that analysed the effect of CACNA1C on brain activity reported opposite effects; for instance the risk allele has been associated with both increased and decreased DLPFC activation 401,409, or even with beneficial effects on structural connectivity in patients ${ }^{423}$.

Moreover, recent data highlight other factors that can modulate the impact of CACNA1C in the brain. It has been observed in animal models that the effect of changes in CACNA1C availability seems to be dependent on the stage of neurodevelopment ${ }^{424}$. 
Also, it has been reported that the effect of early exposure to traumatic events on brain activity is mediated by the $\operatorname{rs} 1006737^{425}$.

With respect to $K C N H 2$, in controls, a positive $S W n$ modulation was observed irrespective to the genotype, which could be interpreted as a network reorganization towards higher efficiency with task performance. Therefore, the opposite pattern observed in patients with the risk allele might be reflecting the allele-associated reduced ability to modulate efficiently the electrical activity during a cognitive task. It is of mention that there were no differences in clinical variables between patients with and without the risk allele. Consequently, the SWn differences observed in patients depending on the $K C N H 2$ polymorphism were not driven by symptoms, treatment dosages or illness duration.

We reported in our previous work ${ }^{117}$ that SWn modulation differed between patients and controls. In that results, patients, as a whole, showed similar SWn modulation patterns than patients with the risk allele in the present report. Thus, the functional network alteration that we previously found might be contributed by $K C N H 2$ genotype risk variant.

The detected effect of the rs3800779 polymorphism is in line with its previously reported functional effects: the $A$ allele has been associated with an increased expression of the $K C N H 2-3.1$ brain isoform ${ }^{416}$, which confers specific electrical properties to the neuron, like higher firing rate and faster deactivation ${ }^{418}$. These properties might affect directly the spread of action potentials and consequently the coordination and communication between distant group of neurons. Therefore, the effect that we observed might be coherent with the neuronal bioelectrical properties associated with this polymorphism, which might influence the functional connectivity patterns in the patient group.

In addition to these functional effects of the rs3800779 $\mathrm{KCNH} 2$, the evaluation of the putative effects on gene expression regulatory mechanisms represents a valuable resource to provide additional meaning to our association data. This can be examined using HaploReg ${ }^{426}$, which is a tool that provides data on the predicted chromatin state of the queried SNP, their sequence conservation in mammals and their effect on regulatory motifs. Interestingly, this SNP is a binding site for the transcription factor 12 
(TFC12), which is described to participate in regulating lineage-specific gene expression. Also, this SNP is predicted to alter a regulatory motif, with the A allele showing lower affinity for a transcription factor (PAX-5) that is involved in neurodevelopment. Such data point towards a putative molecular mechanism underlying the previously described impact of the rs3800779 risk allele on an increased $K C N H 2$ expression and a higher firing rate and faster deactivation ${ }^{418}$. Accordingly, despite not extensive, the accumulated data indicate that the risk variant in $K C N H 2$ affects the biology of the gene in a way that converges on key aspects of the biology of the disorder such as neuron excitability alterations. Thus, the effect of gene variants might be weakly or not detected in relation to the susceptibility for schizophrenia but might be robust in risk-allele carrying populations in relation to specific intermediate phenotypes such as brain functional connectivity phenotypes. In this line, the results from the present study further support the role of genetics in the identification of groups of patients with different connectivity profiles, which would fit with the already suggested existence of schizophrenia subgroups with different biological abnormalities as an explanation of the heterogeneous research findings, the lack of replicability and inaccuracy of current clinical diagnosis ${ }^{6,427}$.

Our study has some limitations, principally the sample size, although Arnedo 58 argued that the low replication rate in genetic studies is not due to small sample sizes but to complex genetic and phenotypic architecture. Accordingly, despite the selected genes and SNPs have been consistently associated with schizophrenia, the polygenic nature of the disorder and the minor effect of common genetic variants limit the power of our sample. Another limitation is the EEG frequency range selected for the study. We limited the functional modulation parameters to the broad band from 4 to $70 \mathrm{~Hz}$ in order to decrease the risk of type I errors and improve interpretably; however, modulation in each frequency band separately could yield relevant results. In summary, the interpretation of these results should be done with caution and further studies are required.

In conclusion, our data suggest that $K C N H 2$ genotype contributes to functional connectivity deficits in schizophrenia patients. We did not find an association between CACNA1C polymorphism and EEG SW properties; however, based on literature, we 
cannot discard its potential effect on connectivity. Further studies with larger samples are required to confirm our results and to extend the knowledge of the implication of KCNH2 and CACNA1C and in schizophrenia electrophysiological related phenotypes. 


\section{Chapter 8}

\section{Discussion}

In this thesis, the hypothetical existence of biological distinctive subgroups among patients with schizophrenia has been explored. We tested two classifying approaches as potential tools that could be used to disentangle the schizophrenia aetiology problem. We shed light on the research replication problem, that is, the inconclusive variability of biological abnormalities found within patients with the same diagnosis.

As a whole, we demonstrated a large heterogeneity in both structural and functional connectivity among schizophrenia patients. We then showed the utility of classifying patients based on biological data rather than through clinical diagnosis. We propose that classifying methods similar to those used in this thesis are necessary to solve the lack of research findings replication, the biological and clinical heterogeneity. Our results support the need to change current psychiatric research perspective towards the underlying biological mechanisms with the aim of achieving better clinical validity.

In the first article, using brain anatomy data from magnetic resonance imaging, we identified a subgroup of approximately $20 \%$ of schizophrenia patients who display an increased cortical curvature (hipergyrification) at the frontal lobe, temporal lobe, cingulate and insula. This subgroup was also characterised by hypometabolism in the caudate and thalamus, a lack of increased metabolism in the putamen (a marker of treatment response) and more persistent negative symptoms.

In the second article, we observed that cortical curvature in PFC was inversely related to the integrity of WM fibres connecting the PFC with the anterior cingulate and temporal lobe, primarily in schizophrenia chronic patients. Therefore, it is plausible that the increased cortical curvature characterising the schizophrenia subgroup is an indirect marker of worse short-range structural connections. It is worth noting that patients without clinical improvement after follow-up had larger values of cortical curvature in 
the superior medial prefrontal cortex and that cortical curvature was inversely associated with cognitive performance but not with antipsychotic treatment, illness duration or PANSS scores.

In the third article, we observed significant decreases of WM integrity, that is, decrease FA in fibres connecting the PFC to the ACC, caudate and hippocampus in a group of 27 patients. The reduced integrity of short cortico-cortical WM fibres (PFC-ACC) was associated with poorer executive function and problem-solving. Surprisingly, better structural connectivity between the PFC and caudate was associated with positive symptoms, but negative symptoms were associated with worse connectivity between PFC and thalamus. Illness duration was related to worse WM connectivity between PFC and subcortical areas, and treatment dosages were not related to WM integrity. These results in both the second and third article reinforced the possible existence of a schizophrenia subgroup with higher cortical curvature, probably due to impaired shortrange connectivity, and reduced cognitive performance.

Considering together the first and third articles, it can be deduced that pooling together patients as a homogeneous group might lead to incorrect conclusions. In the third article, structural abnormalities were found in schizophrenia patients as a whole. Importantly, using neuroanatomical data and a data-driven clustering procedure (not taking in consideration the diagnosis), it is evident that schizophrenia patients do not share the same structural connectivity abnormalities. Rather, these abnormalities are markedly different in the subgroup of patients identified in the first article. Thus, by restricting our analysis to the usual comparison of patients versus controls, we would have assumed that cortical connectivity abnormalities are shared by all schizophrenia patients, which does not seem to be the case.

In the fourth and fifth articles, we observed the utility of selected genetic polymorphisms to classify individuals with or without genetic risk and studied the subgroup's relation to functional connectivity. Specifically, we included genes involved in myelination, excitatory/inhibitory $(E / I)$ balance and neuronal signal transduction contributing to neuronal networks organization. In the first approach (fourth article), we observed that three polymorphisms in the NRG1 gene and related to increased risk of psychosis exert an effect on functional networks modulation during an odd-ball task in 
healthy controls. In the second approach (fifth article), patients and controls were classified depending on polymorphisms located in ether calcium or potassium channels. We observed an effect of $K C N H 2$ rs3800779 polymorphisms, but not CACNA1C rs1006737 polymorphism, on the functional connectivity changes in patients during an auditive odd-ball task. $K C N H 2$ polymorphism separated two groups of schizophrenia patients with significant differences in their functional connectivity modulation efficiency: the first group included patients carrying the risk allele, and the second group included patients non-carrying the risk allele (homozygous for the non-risk allele). Those patients who carry the risk allele ( $A A$ or $A C$ genotype) had an opposite functional network modulation, measured as SWn modulation, in comparison to non-risk allele carriers. Thus, a group of patients with hampered functional connectivity could be distinguished based on genetic variants in potassium channels.

We cannot know whether the higher cortical curvature and the potassium risk-allele carriers subgroups overlap. We might tentatively hypothesize that they are different subgroups considering other work from our team ${ }^{120}$ (not included in this thesis), in which we showed that functional and structural connectivity abnormalities did nor co-occur in the same patients. Indeed, functional connectivity has been previously observed between areas not structurally connected ${ }^{283,428}$.

Therefore, we may have identified two different subtypes of schizophrenia patients, respectively related to structural and functional connectivity alterations. Nevertheless, it is not clear yet how both connectivities are associated, since some previous results supported a mutual relationship ${ }^{122,131,132,134}$, while others do not ${ }^{90,133}$. Furthermore, we cannot discard the possibility that the higher cortical curvature could also be a marker of functional connectivity. In that sense, Nenadic and colleagues observed a widespread cortical thinning (which might reflect functional connectivity disruptions through, for example, loss of dendrites connections) characterising a subgroup of patients with negative symptoms ${ }^{81}$. Additional work reported a correlation between gyrification and functional connectivity ${ }^{429}$. 


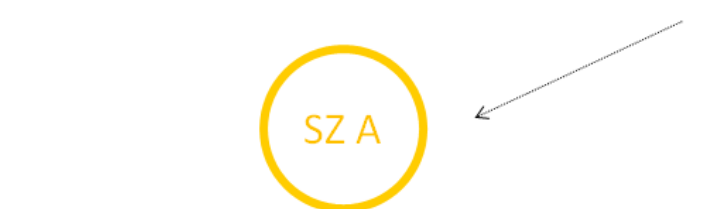

Subgroup characteristics

Higher cortical curvature

Moderate cortical thinning

Hypometabolism in caudate and thalamus

No changes in putaminal metabolism

Impaired short range-connectivity

More negative symptoms

Other posible characteristics?

- Possible worse cognitive capacity
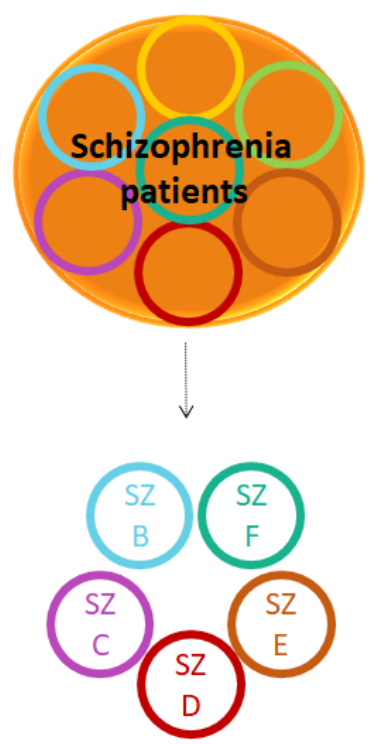

Other posible subgroups

?

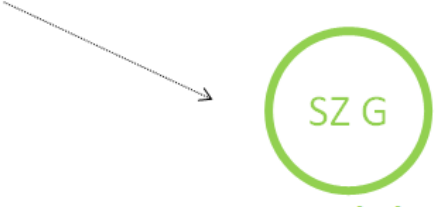

Subgroup characteristics

‡ KCNH2 rs3800779 A allele carriers

* Abnormal connectivity modulation during and auditory oddball task

* No relevant clinical characteristics

Other possible characteristics?

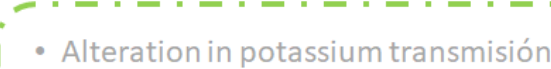

- Increased KCNH2-3.1 isoform expression

- Neuronal specific electrical properties

Figure 8.1. A scheme summarizing the most important findings in this thesis.

\subsection{Subgroups of schizophrenia patients based on structural connectivity}

Concerning curvature, abnormalities have been previously described in schizophrenia patients as a whole. Both hypergyrification in some frontal areas and hypogyrification in the insula, superior temporal, inferior frontal and inferior parietal cortex have been reported ${ }^{430,431}$. In agreement with the subgroup that we identified, increased prefrontal gyrification has been found in high-risk individuals, chronic schizophrenia patients and their relatives $233,432,433$, which indicates a possible genetic contribution to curvature. In fact, polygenic risk score for schizophrenia was positively associated with frontal gyrification in individuals at high familial risk ${ }^{434}$ but negatively associated with inferior parietal curvature in healthy individuals ${ }^{435}$. By contrast, there are also some reports indicating lower prefrontal gyrification in schizophrenia patients 
436-439. The main reason behind these discrepancies is likely due to the previously mentioned biological heterogeneity in schizophrenia, but it could also be related in part to recruitment strategies and sampling selection. For example, increased curvature was described in first episode (FE) schizophrenia 433,440 , which would correspond to our group of FE patients included in the subgroup presenting gyrification abnormalities. However, a decreased curvature has also been reported, including principally FE non-responders to treatment ${ }^{439}$. Finally, another study did not report curvature differences of the ACC in FE schizophrenia patients ${ }^{234}$. FE patients with no curvature abnormalities might correspond to those FE patients included in the subgroup of patients we reported without curvature abnormalities.

Our findings demonstrated that frontal increased curvature is related to abnormal connectivity. This is in agreement with previous data showing that disrupted connectivity during development would be reflected in abnormal gyrification ${ }^{248}$. Indeed, one study used gyrification index as a marker of cortico-cortical connections in subjects at high risk of schizophrenia, schizophrenia patients and controls ${ }^{432}$. Moreover, another study, in relatives of patients with schizophrenia, suggested that frontal cortical curvature is directly related to short-range functional connectivity (connections between the medial and lateral PFC), and inversely related to long-rate functional connections (PFC-thalamus) during a cognitive task ${ }^{429}$. Therefore, we can interpret curvature as a marker of short-range cortico-cortical connections. Thus, the subgroup that we identified based on curvature could be related to a higher white matter dysconnectivity or, alternatively, the gyrification abnormalities might be caused by disrupted connectivity.

So far, we have managed to characterise a subgroup of patients with marked gyrification, probably related to structural cortical connectivity, metabolic disruptions and more severe negative symptoms. This subgroup is notably in agreement with a previously reported subgroup of first episode treatment-naïve patients characterised by widespread WM abnormalities and higher negative symptoms ${ }^{35}$. In our case, the subgroup of patients with higher cortical curvature included almost $50 \%$ of first episode patients despite the fact that they were receiving antipsychotic treatment (the remaining $50 \%$ were chronic schizophrenia patients). In a similar direction, a positive 
correlation between occipital gyrification and negative symptoms was previously described ${ }^{437}$. Also, partially in accordance with our results, another previous study supported a subgroup of patients with higher negative symptoms and widespread cortical thinning ${ }^{81}$. In this case, cortical thinning was moderate in patients with predominantly positive symptoms and mild in those with disorganised symptoms.

As stated in the first article, contrary to our hypothesis, cortical thickness was not the most discriminant variable to classify patients based on neuroanatomical data. Nevertheless, decreased cortical thinning is present in the ACC, right caudal middle frontal, precentral and superior frontal cortex in the aforementioned patients' subgroup identified (with higher cortical curvature), but not in the remaining patients with schizophrenia or bipolar disorder. Those areas were among the areas altered in the subgroup identified by Nenadic and colleagues ${ }^{81}$. However, in our study, treatmentresistant patients were not included. Therefore, we cannot discard the possibility that treatment-resistant patients with negative symptoms could be distinguished by higher cortical thinning.

In contrast to our results, a subgroup of schizophrenia patients with greater WM impairment and longer illness duration, but no differences in negative symptom, has been previously reported ${ }^{441}$. This lack of differences in negative symptoms might be contributed by a gender and age disequilibrium in the subgroups that they identified, specifically the group characterised by structural connectivity disruption had older mean age and included a higher proportion of males

It is interesting to note that patients non-responders to treatment with predominant positive symptoms, in particular, auditory hallucinations, have shown lower gyrification in language areas in comparison to controls ${ }^{235}$. Similarly, patients with delusional infestation showed hypogyrification in the posterior frontal lobe, parietal lobe and right middle temporal cortex ${ }^{442}$. Moreover, lower cortical gyrification values could be associated with positive symptoms in schizophrenia ${ }^{437}$. Therefore, it is likely that higher gyrification is not present in patients with predominantly positive symptoms, which is in agreement with our finding relating higher gyrification to negative but not to positive symptoms. 
In order to validate the patient subgroup we found, it is important to rule out that this subgroup was merely driven by treatment and illness duration. In our case, patients with and without higher cortical curvature do not differ in time of illness duration or antipsychotics doses. Congruently, in the second article, we found that curvature and those clinical variables were unrelated. Moreover, the third article confirmed that treatment was not related to WM integrity, but illness duration was indeed inversely associated with FA in the connections between superior-medial prefrontal cortex and subcortical regions (hippocampus or caudate), but not in short-range cortico-cortical connections. In agreement with our findings, other works showed that gyrification was not correlated with treatment doses or illness duration ${ }^{430}$. Moreover, a tendency towards lower gyrification with age, illness duration ${ }^{431,433,437,438}$ and medication ${ }^{433}$ was described. Considering all these findings, we may conclude that the increased curvature in the identified subgroup is not due to the possible influence of these confusion factors.

It is particularly interesting that the ACC, which has been considered a core region altered in schizophrenia and with homogeneous data among articles ${ }^{28}$, is one of the most important areas involved in the structural dysconnectivity we found in patients. Prefrontal connection with the ACC is possibly partially responsible for the increased PFC curvature we found. However, besides the described is homogeneity, we observed that ACC abnormalities, in curvature, thickness or area, were not present in all patients. Indeed, we observed a decreased left ACC cortical thickness and an increased right ACC cortical curvature in our subgroup of patients with hipergyrification, but not in the remaining schizophrenia patients. This points out that the ACC is not homogeneously altered in all schizophrenia patients.

We observed that higher PFC cortical curvature was associated with worse cognition and that lower WM integrity of short-range connections was correlated with worse executive and problem-solving function. Thus, we hypothesized that our subgroup with marked cortical curvature would have more severe cognitive deficits. Indeed, increased cortical gyrification was previously associated with poorer executive function in firstepisode patients ${ }^{433}$. This finding agrees with a subgroup of patients with severe cognitive deterioration ( $28 \%$ of all patients) who showed greater deficits in grey and white matter, as well as elevated negative symptoms ${ }^{443}$. In the same direction, 
supporting the idea that greater WM impairments are associated with cognitive abnormalities, a subgroup of patients identified with higher cognition impairments was also characterized by WM abnormalities and larger ventricles ${ }^{444}$. On the contrary, patients with mild cognitive impairments showed better life functionality, less severe positive and negative symptoms but more depression ${ }^{445}$, accompanied by GM deficits but not WM disruptions ${ }^{444}$.

\subsection{Subgroups of schizophrenia patients based on genotype and functional connectivity}

In a previous report ${ }^{117}$, we observed functional connectivity abnormalities in schizophrenia patients during the same odd-ball task used in this thesis. Functional connectivity was also assessed using SWn index, which depends on the synchrony and coordination of the brain activity and, therefore, close to the substrate of mental functions altered in schizophrenia. In that article, patients showed higher $S W n$ values at the pre-stimulus window but lower SWn modulation in comparison to controls. On this basis, we wanted to explore the effect of genetic polymorphisms on SWn modulation and thus the possible existence of groups of patients based on modulation deficits.

Firstly, we found that genetic variants associated with schizophrenia risk in NRG1 gene were related to altered SWn values at baseline and its modulation in healthy participants. This means that functional connectivity efficiency is partially mediated by this polymorphism in the population. This makes genetics a promising tool for identifying possible biologically-determined subgroups of schizophrenia patients. Secondly, we observed that $K C N H 2$ rs3800779 polymorphisms, but not CACNA1C polymorphism, was able to discriminate a subgroup of patients with abnormal global functional connectivity modulation (SWn modulation) during the odd-ball task.

The $K C N H 2$ rs3800779 polymorphisms had a significant effect on SWn modulation only in patients but not in controls. Thus, although we did not include patients in the NRG1 study, speculatively, it might be possible that the effect of NRG1 polymorphisms on functional connectivity could be different and even more significant in patients, and hence useful to differentiate subgroups too. Indeed, as the effect of single genetic 
variants is small, polygenic scores, (scores that agglutinate information of several genetic variants associated with schizophrenia risk) may be the best option to explore the existence of subgroups based on genetics ${ }^{446}$, instead of the traditional candidate gene approach.

The lack of association in our results between CACNA1C rs1006737 polymorphism and functional connectivity (SWn at baseline or SWn modulation) could be related to the wide frequency range included in the article (4 to $70 \mathrm{~Hz}$ ). CACNA1C seems to contribute highly to gamma oscillations $(32-64 \mathrm{~Hz}){ }^{447}$, therefore, its possible effect on functional connectivity would be more evident in the high-frequency bands.

As far as we know, there are no previous articles studying the relationship between these polymorphisms and the efficiency of global functional connectivity modulation. There are previous data supporting abnormal functional connectivity during the performance of a P300 or working memory tasks. For example, Quidé and colleagues observed that, while healthy controls increase their functional connections in relation to task performance between PFC, caudate, putamen or parietal lobe, patients did not 448. In fact, patients showed abnormal patterns of increased connectivity between the ventrolateral PFC and putamen, which were inversely associated with working memory performance ${ }^{448}$. Another study found that the association between functional connections and the P300 amplitude was opposite in first-episode patients and healthy controls ${ }^{103}$. In a similar direction, alteration in both task-negative and task-positive networks was described in patients with schizophrenia ${ }^{104}$. Finally, increased connectivity between DLPFC and hippocampus has been consistently found in patients and was related to better task accuracy and decreased symptomatology 104 .

Based on patient's performance on a working memory task, a report showed that the group with better performance exhibited increased functional connectivity involving DLPFC and other areas in comparison to the remaining patients and controls ${ }^{449}$. Indeed, controls with better and worse cognitive performance did not differ in functional connectivity ${ }^{449}$. Therefore, it seems that patients appear to compensate for their disruptions in functional connectivity by increasing some connections. In other words, those patients who are able to compensate for their brain connection alterations may have better cognitive resources. By contrast, those patients with more difficulties in 
compensating for their functional connection disruptions may show a reduced cognitive ability.

On the other hand, since we evidenced in two different studies, that impaired SWn modulation is related to both $K C N H 2$ rs3800779 polymorphism and cognition ${ }^{117}$, and the same polymorphism has been found associated with impaired cognitive performance and lower intelligence quotient 416,417 , it could be possible that the cognitive impairments associated with $K C N H 2$ rs3800779 are mediated by the inability to properly modulate their functional connections (SWn modulation). Indeed, those patients previously described, who had a lower capacity to compensate for their functional connectivity deficits and showed cognitive disruptions, might overlap with the subgroup of patients carrying the $K C N H 2$ risk allele and showing abnormal $S W n$ modulation. While, on the contrary, those patients who were able to compensate their functional connections and showed better cognitive performance, might overlap with the patient subgroup not carrying the risk allele and showing preserved SWn modulation.

In terms of graph parameters, and in agreement with our results, other studies supported abnormal SW properties in schizophrenia, such as lower CLC and higher PL in beta band resulting in lower SW values ${ }^{115}$. In the same vein, decreased SW network properties in beta, alpha and gamma frequency bands were described in a wellfunctioning group of patients ${ }^{126}$. In the latter study ${ }^{126}$, the disruption was higher during cognitive task than in resting state, encouraging the study of subgroups of patients based on functional connectivity during cognitive activity, as we did.

In line with our findings, an interesting study including schizophrenia, schizoaffective disorder and bipolar disorder patients, identified three trans-diagnostic subgroups of patients based on cognition and sensorimotor data (eye movements and electrophysiological measures during paired stimuli task, odd-ball task, saccade task and stop-signalling task) ${ }^{450}$. The first subgroup, called biotype 1 , was characterized by the highest cognitive impairment, worst neurophysiological reactivity to stimuli, including higher inhibition error rates, the lowest amplitude and the poorest target detection. These patients showed widespread GM deficits and the most impaired psychosocial functioning. The second subgroup, called biotype 2, did not show such severe 
alterations, with mild cognitive disturbances, accentuated sensorimotor reactivity (including normal to higher amplitude), highest stimuli-unrelated EEG activity and normal target detection. This second group also presented widespread GM deficits, but less marked, and mild impairments on the psychosocial function. Finally, the third subgroup, called biotype 3, was the most similar to healthy controls. Their cognitive function was preserved, with modest sensorimotor disruption, discrete GM reductions, specifically located in anterior limb areas, and good psychosocial functioning. The first and second groups did not differ on clinical symptoms, but the third group had significative lower positive and negative symptoms scores. Interestingly, these characteristics, though more moderate, were found in the relatives of each group of patients. The authors argued that, possibly, the subgroups one and two could be more related to familiar genetic risk as well as related to calcium and potassium channels disruptions, reinforcing, in agreement with our studies, the utility to study subgroups of patients based on polymorphisms in these voltage-gated channels. On the other hand, the third subgroup may be more related to environmental hazards, epigenetics or spontaneous mutations.

In a later study of the same research group ${ }^{451}$, fMRI was used to measure restingstate functional networks between subgroups. This study found that the three biotypes showed dysconnectivity in relevant networks, but with it being more predominant in individuals from the biotype one and two. They also observed that those functional resting state networks were closely related to cognitive performance, but not as closely related to the sensoriomotor reactivity as they had expected. In conclusion, fMRI connectivity and electrophysiological variables during auditory task seemed to be complementary rather than related.

Taking this into account, the subgroup of patients carrying the $K C N H 2$ risk allele might be consistent with the biotype one or even two in those previous reports. Risk allele carriers had abnormal functional connectivity modulation while biotypes one and two had impaired electrophysiological response to stimuli and connectivity disruptions. On the contrary, the biotype three, with less severe impairments, could be close to our subgroup of patients not carrying the risk allele who showed intact SWn modulation. In those previous studies, genetic information was not included but it could be speculated 
that the biotype one and/or two might show abnormalities in neuron ion channels, such as those within the $K C N H 2$ gene. It could be possible that reduced functional connectivity adjustment during a cognitive task is partially determined by the presence of risk polymorphisms in genes involved in neuronal electric signalling transduction and excitatory/inhibitory potentials. This support the utility of classifying patients based on their genes to study differences in functional connectivity within schizophrenia.

Finally, regarding the patient subgroup we identified who carry the $K C N H 2$ risk allele, it is also important to rule out that their connectivity characteristics were driven by clinical confounders. First, we observed no significant differences in symptomatology between risk-allele carriers and non-carriers, with the only mention of a non-significant slight tendency towards more negative symptoms with regard to risk allele carriers. Also, we did not observe an effect of symptoms on SWn in our previous report ${ }^{117}$. However, we cannot discard a possible relation between functional connectivity and symptoms, as previous articles did observe such association. For example, an altered pattern of functional connectivity in schizophrenia patients, measured as fast-frequency band imaginary coherence between PFC and occipital areas, was associated with symptoms in post-stimulus specific time windows ${ }^{105}$. Also, CLC and PL, yielding abnormal SW, were associated with negative and cognitive symptoms, although in opposite directions, negatively in the case of CLC and positively in the case of $\mathrm{PL}^{115}$. Sample size may explain the lack of significant association in our studies between symptoms and functional connectivity. In addition, we did not observe differences in illness duration and treatment between $\mathrm{KCNH} 2$ risk polymorphism carriers and non-carriers. Moreover, in our previous report, $S W n$ values were not related to months of illness duration or antipsychotic doses ${ }^{117}$. In agreement with this, a correlation between illness duration and treatment was not found with either $\mathrm{CLC}$ or $\mathrm{PL}{ }^{126}$. Thus, global functional connectivity may not be affected by dosage of treatment or illness duration and the different modulation in risk-allele carriers we found is probably not due to the effect of antipsychotics or chronicity. 


\subsection{Limitations of the study}

This thesis has some limitations that should be noted:

- All patients included were under antipsychotic medication. Therefore, it was not possible to completely rule out the effects of treatment on the measured variables. Nevertheless, the possible relations between antipsychotic dosages and relevant variables were studied using regressions or correlations analyses. Also, in the first article, the inclusion of patients with bipolar disorder aimed to discard possible treatment effects on neuroanatomical variables, as the majority were also under antipsychotic treatment.

- Cumulative dosages of antipsychotics could not be calculated and therefore it was not possible to disentangle the possible effect of long-time treatment over connectivity difficult.

- In the articles included, sample sizes varied from 27 to 121 schizophrenia patients and from 27 to 144 healthy controls. Also, in those articles considering different study variables, some of the measurements were not available for all individuals (i.e. metabolic activity and P300 data in the first study and cognitive data in the second and third study). Larger samples sizes would increase the study power and help in identifying connectivity alterations and subgroups of patients. Remarkably, the first article, which is the most important in terms of subgroup identification, had the largest sample size.

- The data-driven method selected for subgroup's identification yielded one specific classification result, nevertheless, the result could vary slightly depending on the data-driven method. In other words, the classifying result is not fixed and unique as it would depend on, first, the variables included and, second, the classifying method (i.e. other algorithms or machine learning)

- Cognition was not included in all the articles of this thesis because it was not available (case of the first article), or to avoid blurring the principal aim of the studies (the two last articles). However, in those studies, the inclusion of cognition would have helped validating the patient subgroups.

- Diffusion magnetic resonance cannot identify different processes damaging WM fibres. Thus, we can not determine whether the decreased fractional anisotropy 
observed in patients was due to abnormal myelin compaction, demyelination, or decreases in the axons/fibres number. Moreover, dMRI cannot properly identify places with fibres crossing.

- Streamlines count variable (included in the third study) was assumed to be a measure of the number of fibres although, actually, its exact meaning it is still unknown.

- Electroencephalogram was recorded using 17 electrodes. Nevertheless, a larger number of electrodes may have improved graph-parameters calculations.

- $\quad S W$ parameters were calculated at five different frequency bands in the NRG1 study, but in a global frequency band, from 4 to $70 \mathrm{~Hz}$, in the $K C N H 2$ and CACNA study. Therefore, the possible effect of the selected genes may have been different depending on the frequency band selected.

- Single candidate gene studies may not be the most effective method of disentangling the genetic effects on functional connectivity, as this is probably mediated by thousands of possible genetic variants.

- This thesis did not include longitudinal analyses to observe the changes in connectivity and the validity of subgroups over time. 


\section{Chapter 9}

\section{Conclusions}

\subsection{Main conclusions of the study}

According to the aims, hypotheses and results, the main conclusions of this thesis are the following:

i. Patients with schizophrenia diagnosis could be classified by using structural data obtained from magnetic resonance and a data-driven classification approach. A specific subgroup of patients was distinguishable from the rest of patients with schizophrenia, patients with bipolar disorder and healthy controls based on markedly higher mean cortical curvature.

ii. The schizophrenia subgroup with higher mean cortical curvature was additionally characterised by decreased metabolic rates in thalamus and cingulate, a lack of the expected, treatment-related, increased metabolic rates in putamen and no improvement of negative symptoms.

iii. Cortical curvature in the prefrontal cortex could be understood as an indirect measure of structural connectivity mainly in short-range connections. Higher values of prefrontal cortical curvature were associated with lower fractional anisotropy in connections between the prefrontal cortex and both anterior cingulate and superior temporal cortices.

iv. Schizophrenia patients showed, in comparison to healthy controls, altered integrity of white matter fibres connecting prefrontal cortex with cingulate, thalamus, caudate and hippocampus. Importantly, higher structural impairment between prefrontal cortex and cingulus was associated with reduced cognitive performance in motor speed and problem solving, and higher structural impairments between prefrontal cortex and subcortical regions correlated with higher positive symptoms 
scores and illness duration. Pharmacological treatment was not associated with structural connectivity in any case.

v. Selected polymorphisms associated with schizophrenia risk and located in genes involved in myelinization, synaptic transmission or excitatory/inhibitory balance (NRG1, CACNA1C and $K C H N 2$ ) were associated with differences in the modulatory efficiency of global functional connectivity in healthy controls and patients.

vi. The rs6468119, rs6994992 and rs7005606 polymorphisms in NRG1 gene were associated with small-worldness modulation (change of global functional connectivity efficiency) in theta, alpha and beta 1 frequency bands in healthy controls.

vii. The rs3800779 polymorphism located in $K C H N 2$ gene was related to differences in the small-worldness modulation in the global frequency band in patients but not in healthy control subjects. Importantly, carrying the risk allele (A) distinguished a group of patients with abnormal modulation of small-world values during a working memory task (P300).

viii. The rs1006737 polymorphism located in CACNA1C gene was not associated with small-worldness either at baseline or its modulation and could not differentiate any subgroup of patients.

ix. Finally, as a general conclusion, data from structural and functional connectivity, as well as genetic polymorphisms proved to be useful in exploring the existence of specific patient subgroups within schizophrenia with different patterns of brain connectivity.

\subsection{Future research lines}

In the near future, we would like to continue the following research lines already started during my thesis:

- To continue exploring the utility of classificatory approaches, both data-driven and genetic based.

- To replicate and validate our result of a subgroup of patients characterised by larger curvature by using a completely different and larger sample, as well as by employing different data-driven approaches. 
- To replicate and validate the genetic effect and the utility of genetic-based classification using NRG1, KCNH2 and CACNA1C polymorphisms on functional connectivity in a different and larger sample.

- To characterise the identified subgroups in more depth by using other types of variables such as social cognition, childhood trauma, markers of inflammation and/or epigenetics.

- To study the possible existence of other subgroups of patients characterized by functional connectivity abnormalities by using a data-driven approach similar to that applied to the structural connectivity.

- To include a larger number of polymorphisms related to the risk of mental diseases and involved in key processes for brain connectivity such as myelination, neurotransmitter signalling, E/I balance, synapsis formation and maintenance as well as synaptic pruning.

- To calculate polygenic risk scores and observe its effect over both structural and functional connectivity, and to analyse its power as a potential classificatory tool.

- To include patients diagnosed with bipolar disorder and/or other psychiatric conditions in order to evaluate the existence of biologically valid subgroups of patients cross the current diagnostic boundaries.

- To analyse EEG graph parameters in different frequency bands in order to observe the functional connectivity divergences at several ranges of the electrical spectrum.

- To study alternative EEG tasks to measure, for example, resting state activity or activity associated with other cognitive processes.

- To include additional neuroimaging techniques that would help in understanding the altered functions in patients; for example, fMRI to assess functional connectivity with a better spatial resolution, or transcranial magnetic stimulation in order to manipulate inhibitory capacity and observe its effects on connectivity, cognition and clinical variables.

- To discard the effect of treatment including naïve patients and relatives, as well as other diagnostic pathologies being treated with antipsychotics. 
- To perform longitudinal studies to observe the connectivity changes over time and the effects of chronicity and treatment cumulative exposure. Also, to assess the validity of patient subgroups over time. 


\title{
Appendix A
}

\section{Student contributions to each publication}

\author{
Chapter 3: Identification of two clusters within schizophrenia with different \\ structural, functional and clinical characteristics \\ During the performance of this study, I processed the MRI images, calculated all the \\ MRI data: cortical curvature, thickness, area and subcortical volume; and created the \\ working database. Moreover, I calculated the sociodemographic statistics and designed \\ all the tables in the paper. Finally, I also participated in writing and revising the \\ manuscript.
}

Chapter 4: Biological and cognitive correlates of cortical curvature in schizophrenia

In this publication, I assisted in the MRI acquisition, processed the MRI data to obtain the cortical curvature measures, extracted the appropriate data from the connectivity matrices, and created the working database. Furthermore, together with V. Molina and R. de Luis, I participated in the statistical analyses, designed the tables and figures in the article, and wrote the manuscript.

Chapter 5: Alterations in prefrontal connectivity in schizophrenia assessed using diffusion magnetic resonance imaging

To perform this work, I assisted in the MRI acquisition and data processing, extracted the appropriate data from the connectivity matrices previously calculated by R. de Luis, and created the database. Similar to previous work, V. Molina, R. de Luis and I undertook the statistical analyses, created the tables and figures in the article, and wrote the manuscript. 
Chapter 6: Variation at NRG1 genotype related to modulation of small-world properties of the functional cortical network

In the elaboration of this publication, I helped in the EEG acquisition, created the working database and performed the statistical analyses. I configured the tables and figures in the article and wrote the manuscript in collaboration with V. Molina and A. Palomino.

Chapter 7: Analysis of KCNH2 and CACNA1C schizophrenia risk genes on EEG functional network modulation during an auditory odd-ball task

During this work, I helped in the EEG acquisition and the genotyping procedure. I also created the working database. M. Fatjó-Vilas and I undertook the statistical analyses, designed the tables and figures in the article and wrote the manuscript under the supervision of V. Molina. 


\section{Appendix B}

\section{Spanish summary / Resumen en}

\section{castellano}

\section{A.1. Introducción}

La esquizofrenia es una enfermedad neuropsiquiátrica grave, que afecta al pensamiento, las emociones y el comportamiento, determinando una gran afectación de la calidad de vida del paciente y quienes le rodean y una disminución de la esperanza de vida en torno a 20 años. Por otro lado, sus costes económicos son elevadísimos: un estudio calculó que los costes directos e indirectos asociados a la enfermedad en España en el año 2002, eran alrededor de 1970 millones de euros anuales, siendo los gastos directos un $2,7 \%$ del presupuesto anual del sistema público de salud.

La esquizofrenia se caracteriza por la presencia de síntomas positivos, negativos y cognitvos. Está presente en todo el mundo con una prevalencia e incidencia muy similar, entre el $0,3-0,7 \%$ y el $10,2-22,0 \%$ o respectivamente. El inicio de la enfermedad se da entre los 20 y 30 años, pudiendo comenzar tras un periodo de sintomatología atenuada conocido como pródromo. El pronóstico varía mucho entre los pacientes, pero al menos la mitad son pacientes crónicos, algunos con gran incapacidad, que requieren atención sanitaria y farmacológica a lo largo de la vida.

La etiopatogenia de la esquizofrenia es actualmente desconocida, las evidencias apuntan a que es la suma de factores genéticos y factores ambientales que conceden vulnerabilidad, lo que desencadena la enfermedad. A pesar del gran número de investigaciones científicas, el dinero y el esfuerzo invertido en entender las enfermedades mentales, hasta la fecha no se ha podido identificar de modo fiable una sola anomalía genética, cerebral, biológica o de otro tipo característica de cualquiera de 
las formas clínicas de la psicosis. Es decir, actualmente no existe ningún fenotipo biológico que ayude en el diagnóstico o la predicción de respuesta al tratamiento.

Los criterios diagnósticos vigentes actualmente llevan a que pacientes con grandes diferencias clínicas reciban el mismo diagnóstico y tratamiento o que, por el contrario, pacientes con distintos diagnósticos (esquizofrenia, trastorno bipolar, depresión mayor con síntomas psicóticos...) compartan síntomas, evolución, respuesta farmacológica o perfiles cognitivos. Esto hace que el grupo de pacientes con un mismo diagnóstico sea ampliamente heterogéneo. Por lo tanto, tratando a los pacientes con esquizofrenia como un grupo homogéneo se impide la identificación de sustratos biológicos que lo expliquen y diferencien de otras enfermedades mentales. De hecho, los hallazgos hasta la fecha indican que pacientes con diagnósticos distintos comparten tanto variaciones genéticas como anomalías cerebrales. Y a su vez, pacientes con el mismo diagnóstico pueden tener distinta variaciones genética y alteraciones cerebrales. La búsqueda de grupos de pacientes que compartan alteraciones biológicas comunes sin tener el cuenta el diagnóstico inicial puede ayudar, por un lado, a entender mejor qué ocurre en el cerebro de los pacientes, y, por otro, a buscar métodos diagnósticos más cercanos a la etiopatogenia biológica que ayuden a mejorar los tratamientos y la calidad de vida de los pacientes.

Esta tesis incluye datos procedentes de varias técnicas que estudian los procesos biológicos cerebrales, analizando su relación con la esquizofrenia y las posibles alteraciones biológicas en este trastorno. En otras palabras, mediante un análisis de las alteraciones cerebrales y genéticas en pacientes con esquizofrenia y en comparación con la población sana, se planteó definir patrones de alteración de la conectividad estructural y funcional independientemente del diagnóstico clínico inicial..

Para ello, se partió del estudio anatómico del cerebro utilizando imágenes de resonancia magnética (RM) que cuantifican las regiones cerebrales y la integridad de las conexiones estructurales. La idea inicial era la identificación de grupos de pacientes con distintas alteraciones, para lo que se estudió la relación entre medidas corticales y de conectividad estructural y se analizó esa conectividad estructural en los pacientes. Posteriormente, se evaluó la relación entre variantes genéticas relacionadas con un aumento del riesgo de la esquizofrenia e implicadas en funciones relevantes con las 
alteraciones de las conexiones cerebrales funcionales. Y, por último, se estudió la capacidad de estas variantes genéticas para identificar subgrupos de pacientes con distintas anomalías de la conectividad funcional.

\section{A.2. Hipótesis y Objetivos}

En base a la literatura actual y teniendo en cuenta la gran heterogeneidad dentro de la esquizofrenia, la hipótesis principal de esta tesis es la existencia de distintos subgrupos de pacientes con diferentes alteraciones de la conectividad estructural y funcional dentro de la esquizofrenia. Estos grupos podrían determinarse con métodos estadísticos de agrupamiento que no tienen en cuenta los diagnósticos o en base a las variantes genéticas presentes en cada individuo.

Partiendo de esta hipótesis, los objetivos de esta tesis doctoral son los que se relacionan a continuación:

- Estudiar la posible existencia de grupos de pacientes dentro de la esquizofrenia, en función de datos de anatomía cerebral (volumen, grosor y curvatura cortical y volumen subcortical), junto con métodos estadísticos de clasificación.

- Validar los subgrupos encontrados en función de otros parámetros biológicos.

- Relacionar las alteraciones anatómicas cerebrales con alteraciones de la conectividad estructural basadas en el estudio de la integridad de los tractos de materia blanca.

- Analizar las diferencias de conectividad estructural de regiones prefrontales entre pacientes y controles.

- Evaluar si variantes genéticas relacionadas con un mayor riesgo de la esquizofrenia e implicadas en la transmisión sináptica y el balance excitación/inhibición, se relacionan con la conectividad funcional en pacientes y en controles sanos.

- Estudiar la capacidad de esas variantes genéticas para distinguir grupos de pacientes con distintas alteraciones de la conectividad funcional. 


\section{A.3. Materiales y Métodos}

Esta tesis doctoral está formada por cinco artículos científicos, cuatro de ellos ya publicados y uno en proceso de revisión. Se han incluido datos de controles sanos, pacientes con diagnóstico de esquizofrenia y pacientes con trastorno bipolar. Los sujetos fueron seleccionados según criterios específicos de inclusión y exclusión. Los pacientes fueron diagnosticados siguiendo los criterios del DSM-IV y V, estaban bajo tratamiento y su sintomatología positiva y negativa se cuantificó mediante la escala Positive and Negative Syndrome Scale (PANSS). El tamaño muestral varía en función de cada artículo, desde 21 hasta 121 pacientes con esquizofrenia, desde 27 hasta 144 controles sanos y 22 pacientes con trastorno bipolar.

En el primer trabajo incluido en esta tesis, se analizaron las imágenes de RM (T1) de controles sanos y pacientes con esquizofrenia y trastorno bipolar mediante el programa FreeSufer. Se obtuvieron datos de volumen, grosor y curvatura cortical y volumen subcortical. Además, se incluyeron en los análisis estadísticos, datos electroencefalográficos de amplitud y latencia de la onda P300 y datos de consumo de glucosa cerebral adquiridos con tomografía de emisión de positrones.

En el segundo y tercer trabajo, se utilizaron imágemes de resonancias magnéticas realizadas en la Unidad de Resonancia Magnética de 3 Teslas de la Universidad de Valladolid. Por cada sujeto se adquirieron dos secuencias: una imagen estructural T1 y una imagen de tensores de difusión (DTI). Además, en estos trabajos se evaluó la capacidad cognitiva y el coeficiente intelectual de los sujetos participantes mediante los siguientes test: WAIS-III, BACS y WCST. Las imágenes T1 se procesaron mediante el programa FreeSurfer para obtener, por un lado, datos de curvatura cortical y, por otro, una matriz de 84 regiones. Por otra parte, la imágenes de tensores de difusión fueron procesadas en colaboración con el grupo de Laboratorio de Procesado de Imagen de la Universidad de Valladolid, obteniendo junto a las matrices anatómicas, una matriz de conexiones entre cada par de regiones cerebrales (en total 84 regiones).

En el cuarto y quinto trabajo, se obtuvieron muestras sanguíneas de los sujetos, de las que se aisló ADN. A partir de este ADN, se genotiparon polimorfismos de nucleótido único (variantes alélicas de un solo nucleótido presentes en al menos el $1 \%$ de la 
población) relacionados con un mayor riesgo de esquizofrenia y localizados en genes con funciones relevantes para la mielinización, el balance excitatorio e inhibitorio y la transmisión sináptica. En concreto, se analizaron tres polimorfismos en el gen de la neuregulina 1 (NRG1), un polimorfismo en el gen de la subunidad alfa $1 \mathrm{C}$ del canal de calcio dependiente de voltaje (CACNA1C) y otro polimorfismo localizado en un gen de una subunidad de un canal de potasio dependiente de voltaje, subfamilia $\mathrm{H}$ y miembro $2(K C N H 2)$. En el proceso de genotipación se utilizaron sondas $\operatorname{TaqMan}^{\mathrm{TM}} \mathrm{y}$ discriminación alélica basada en fluorescencia.

Por otro lado, a los sujetos participantes en ambos estudios se les realizó un registro electroencefalográfico durante una tarea auditiva de potenciales evocados (P300). Esta tarea consistía en la presentación aleatoria de 3 estímulos sonoros de corta duración y distinta probabilidad de presentación. Uno de esos tonos era un estímulo diana, cuando sonaba este estímulo los sujetos respondían presionando un botón. Los registros electroencefalográficos fueron analizados en colaboración con el grupo de Investigación Biomédica de la Universidad de Valladolid. El procesado se realizó siguiendo los siguientes pasos: i) filtrado de los registros, ii) eliminación de artefactos oculares (ICA) y motores, iii) transformación de la señal a tiempo-frecuencia mediante la transformada Wavelet, iv) segmentación de los registros (desde -300 ms hasta 700 ms tras el estímulo diana) y división en dos ventanas temporales: ventana pre-estímulo (desde -300 ms hasta el inicio del tono diana) y ventana de respuesta (desde los 150 ms hasta los 450 ms tras el tono diana), v) cálculo de la coherencia de la señal entre cada par de electrodos y de las matrices de conectividad entre electrodos, y vi) cálculo de parámetros de red en cada ventana temporal. Finalmente, se obtuvo un parámetro de eficiencia de la red llamado "Small-world" (SW) que cuantifica la similitud de la red a una red ideal eficiente en la transmisión de información. Los valores de SW se restaron entre la ventana de respuesta y la de pre-estímulo para obtener el valor de la modulación de la red de conexiones ante el estímulo diana.

Por último, para cada trabajo se realizó un análisis estadístico específico. 


\section{A.4. Resultados}

En primer lugar, se identificó un subgrupo de pacientes con esquizofrenia caracterizado por mayores valores de curvatura cortical respecto al resto de pacientes con esquizofrenia, los pacientes con trastorno bipolar y los controles sanos. Este subgrupo además presentaba una ligera disminución del grosor cortical, más alteraciones del consumo de glucosa cerebral y menor mejoría en los síntomas negativos.

Posteriormente, se encontró que mayores valores de curvatura cortical en regiones prefrontales se relacionaban con peor integridad de las conexiones estructurales (menos anisotropía fraccional en los correspondientes tractos de materia blanca) entre la corteza prefrontal con el cíngulo anterior y la corteza prefrontal con la corteza temporal superior. De esta manera, la curvatura cortical prefrontal puede interpretarse como una medida indirecta de las conexiones estructurales a corta distancia. Además, esta curvatura cortical se relacionaba negativamente con las puntuaciones cognitivas y la mejoría clínica.

En el siguiente trabajo se estudiaron las conexiones estructurales, el número y su integridad, entre regiones prefrontales, cíngulo anterior y áreas subcorticales de interés en pacientes con esquizofrenia. El resultado indicaba que los pacientes en su conjunto tenían peor integridad de muchas de estas conexiones, pero esta peor integridad no siempre iba acompañada de una disminución en el numero de fibras. Las conexiones prefrontales con el cíngulo anterior se relacionaban positivamente con la cognición, es decir, a mayor número de fibras y más integridad, mejor cognición. También se observó que el tratamiento no influía en la conectividad estructural, aunque la duración de la enfermedad sí parecía tener un efecto negativo, sobre todo en las conexiones prefrontales con áreas subcorticales.

En la segunda parte de la tesis se evaluó la relación de las variaciones genéticas relacionadas con el riesgo de esquizofrenia y la capacidad de modular adecuadamente las conexiones cerebrales funcionales (medidas con la variable SW) durante una tarea cognitiva. Los polimorfismos localizados en el gen de la NRG1 influían en la modulación de la red cerebral en sujetos sanos. Además, incluyendo pacientes y controles, se 
observó que el polimorfismo en el canal de calcio (CACNA1C) no tenía efecto sobre la modulación del SW. Sin embargo, existía un efecto significativo del polimorfismo del canal de potasio $(K C N H 2)$ sobre la modulación del $S W$. Aquellos pacientes portadores del alelo de riesgo para esquizofrena del polimorfismo en $K C N H 2$ tenían una modulación del SW contraria al resto de pacientes y los controles. En otras palabras, los pacientes portadores del alelo de riesgo disminuían la eficiencia de la red de conexiones al responder a la tarea, mientras que los pacientes no portadores y los controles aumentaban la eficiencia.

\section{A.5. Discusión}

Los resultados de los tres primeros artículos apuntan hacia la existencia de un subgrupo de pacientes dentro de la esquizofrenia con más curvatura cortical, que parece reflejar alteraciones en la integridad de los tractos de materia blanca subyacentes. Además, este subgrupo se caracteriza por alteraciones del consumo cerebral de glucosa, más síntomas negativos y probablemente peor capacidad cognitiva. Es importante destacar que el tratamiento no determina las diferencias observadas en este subgrupo. Estos resultados parecen coherentes con los trabajos de otros autores. Así, en pacientes de primer episodio sin tratamiento, se observó un grupo con menor integridad de las conexiones estructurales y más síntomas negativos. También se ha identificado un posible grupo de pacientes caracterizado por más síntomas negativos y menor grosor cortical. En la misma línea, se han encontrado grupos de pacientes con peor capacidad cognitiva y alteraciones asociadas de la materia blanca.

En la segunda parte, los resultados demuestran la utilidad de las variantes genéticas para identificar subgrupos de pacientes, en esta ocasión con alteraciones de la conectividad funcional. El polimorfismo localizado en el gen $K C N H 2$ parece identificar dos grupos relacionados con esa conectividad: pacientes con menor capacidad de modular adecudamente sus conexiones cerebrales durante una tarea cognitiva y sujetos sin esa alteración. El alelo de riesgo en este polimorfismo se ha relacionado con el aumento de la expresión de una isoforma de la proteína que se encuentra en mayor proporción en el cerebro y que cambia las propiedades bioeléctricas del canal de 
potasio. Por tanto, el efecto observado en los pacientes portadores del alelo de riesgo de $K C N H 2$ podría deberse a los cambios electrofisiológicos en este canal, que afectaría a la coordinación de la actividad eléctrica neuronal.

\section{A.6. Conclusiones}

Teniendo en cuenta los objetivos e hipótesis iniciales y los resultados obtenidos, las conclusiones principales de esta tesis doctoral son:

I. Los pacientes con diagnóstico de esquizofrenia pueden ser clasificados en subgrupos en función de sus datos anatómicos y procesos de clasificación que no tienen en cuenta los grupos a priori o en función de variantes genéticas.

II. Se identificó un grupo de pacientes con esquizofrenia caracterizado por mayores valores de curvatura cortical, alteraciones del consumo de glucosa cerebral y falta de mejoría en síntomas negativos.

III. La curvatura cortical prefrontal se relaciona con la integridad de las conexiones cortico-corticales principalmente de corta distancia.

IV. Los pacientes con esquizofrenia mostraban peor integridad y cambios en el número de conexiones en los tractos de mielina que conectaban la corteza prefrontal con el cíngulo anterior, el tálamo, el hipotálamo y el núcleo caudado. La peor conectividad prefrontal con el cíngulo anterior, se relacionaba con peor función cognitiva.

V. En sujetos sanos, los polimorfismos rs6468119, rs6994992 y rs7005606 en el gen de la NRG1 están asociados con la modulación de las conexiones funcionales cerebrales durante la realización de una tarea cognitiva.

VI. El polimorfismo rs1006737 localizado en el gen CACNA1C, no está asociado con la modulación de las conexiones funcionales, ni en pacientes ni en controles.

VII. El polimorfismo rs3800779 localizado en el gen $K C H N 2$, se relaciona con la modulación de las conexiones funcionales en pacientes. En concreto, los pacientes portadores del alelo de riesgo disminuyen la eficiencia de sus conexiones cerebrales ante un estímulo, mientras que los pacientes no portadores y los controles la aumentan. 
Estos hallazgos confirman la hipótesis de partida de esta tesis doctoral, que sugería que la esquizofrenia no es una enfermedad única, sino un síndrome que engloba pacientes con diferentes alteraciones biológicas. Esto respalda la necesidad de cambiar la perspectiva de investigación dentro de la salud mental, replanteando la validez de los diagnósticos actuales. 



\section{Bibliography}

1. van Os, J. \& Kapur, S. Schizophrenia. The Lancet 374, 635-645 (2009).

2. McGrath, J., Saha, S., Chant, D. \& Welham, J. Schizophrenia: A concise overview of incidence, prevalence, and mortality. Epidemiologic Reviews 30, 67-76 (2008).

3. GBD 2015 Disease and Injury Incidence and Prevalence Collaborators, G. 2015 D. and I. I. and P. Global, regional, and national incidence, prevalence, and years lived with disability for 310 diseases and injuries, 1990-2015: a systematic analysis for the Global Burden of Disease Study 2015. Lancet (London, England) 388, 1545-1602 (2016).

4. Oliva-Moreno, J., López-Bastida, J., Osuna-Guerrero, R., Montejo-González, A. L. \& Duque-González, B. The costs of schizophrenia in Spain. Eur. J. Heal. Econ. 7, 182-188 (2006).

5. Hallak, J., de Paula, A., Chaves, C., Bressan, R. \& Machado-de-Sousa, J. An Overview on the Search for Schizophrenia Biomarkers. CNS Neurol. Disord. - Drug Targets 14, 9961000 (2015).

6. Molina, V. \& Blanco, J. A. A proposal for reframing schizophrenia research. J. Nerv. Ment. Dis. 201, 744-52 (2013).

7. Tiihonen, J. et al. 11-year follow-up of mortality in patients with schizophrenia: a population-based cohort study (FIN11 study). Lancet 374, 620-627 (2009).

8. Laursen, T. M., Nordentoft, M. \& Mortensen, P. B. Excess Early Mortality in Schizophrenia. Annu. Rev. Clin. Psychol. 10, 425-448 (2014).

9. American Psychiatric Association. DSM-V. Diagnostic and Statistical Manual of Mental Disorders (5th ed.) (2013).

10. World Health Organization. The ICD-10 Classification of Mental and Behavioural Disorders. Int. Classif. 10, 1-267 (1992).

11. Keshavan, M. S., Nasrallah, H. A. \& Tandon, R. Schizophrenia, 'Just the Facts' 6. Moving ahead with the schizophrenia concept: From the elephant to the mouse. Schizophrenia Research 127, 3-13 (2011).

12. Molina, V. La psicosis: Ideas sobre la locura. (Biblioteca Nueva, 2012).

13. Beck, K. et al. The practical management of refractory schizophrenia - the Maudsley Treatment REview and Assessment Team service approach. Acta Psychiatr. Scand. 130, 427-438 (2014).

14. Nenadic, I., Gaser, C. \& Sauer, H. Heterogeneity of brain structural variation and the structural imaging endophenotypes in schizophrenia. Neuropsychobiology 66, 44-49 (2012).

15. Gillespie, A. L., Samanaite, R., Mill, J., Egerton, A. \& MacCabe, J. H. Is treatment-resistant schizophrenia categorically distinct from treatment-responsive schizophrenia? A systematic review. BMC Psychiatry 17, (2017).

16. Molina, V. et al. Differential clinical, structural and P300 parameters in schizophrenia patients resistant to conventional neuroleptics. Prog. Neuro-Psychopharmacology Biol. Psychiatry 32, 257-266 (2008).

17. Demjaha, A., Murray, R. M., McGuire, P. K., Kapur, S. \& Howes, O. D. Dopamine synthesis capacity in patients with treatment-resistant schizophrenia. Am. J. Psychiatry 169, 1203- 
1210 (2012).

18. Demjaha, A. et al. Antipsychotic treatment resistance in schizophrenia associated with elevated glutamate levels but normal dopamine function. Biological Psychiatry 75, (2014).

19. Yilmaz, Z. et al. Antipsychotics, dopamine D2 receptor occupancy and clinical improvement in schizophrenia: A meta-analysis. Schizophr. Res. 140, 214-220 (2012).

20. Seeman, P. Clozapine, a fast-off-D2 antipsychotic. ACS Chemical Neuroscience 5, 24-29 (2014).

21. Carpenter, W. T. \& Kirkpatrick, B. The heterogeneity of the long-term course of schizophrenia. Schizophr. Bull. 14, 645-652 (1988).

22. Jobe M., T. H. and H. Long-term outcome of patients with schizophrenia: a review. Longterm outcome of patients with schizophrenia: a review 50, SP-892-900 (2005).

23. Harrison, G. et al. Recovery from psychotic illness: A 15- and 25-year international followup study. Br. J. Psychiatry 178, 506-517 (2001).

24. Molina, V. et al. Subcortical and cortical gray matter differences between Kraepelinian and non-Kraepelinian schizophrenia patients identified using voxel-based morphometry. Psychiatry Res. - Neuroimaging 184, 16-22 (2010).

25. Dazzan, P. et al. Magnetic resonance imaging and the prediction of outcome in firstepisode schizophrenia: A review of current evidence and directions for future research. Schizophr. Bull. 41, 574-583 (2015).

26. Manrique-Garcia, E. et al. Prognosis of schizophrenia in persons with and without a history of cannabis use. Psychol. Med. 44, 2513-2521 (2014).

27. Valencia, M. et al. Predicting functional remission in patients with schizophrenia: A crosssectional study of symptomatic remission, psychosocial remission, functioning, and clinical outcome. Neuropsychiatr. Dis. Treat. 11, 2339-2348 (2015).

28. Brugger, S. P. \& Howes, O. D. Heterogeneity and Homogeneity of Regional Brain Structure in Schizophrenia: A Meta-analysis. JAMA psychiatry 74, 1104-1111 (2017).

29. Wang, A. K. \& Miller, B. J. Meta-analysis of Cerebrospinal Fluid Cytokine and Tryptophan Catabolite Alterations in Psychiatric Patients: Comparisons between Schizophrenia, Bipolar Disorder, and Depression. Schizophr. Bull. 44, 75-83 (2018).

30. Vitolo, E. et al. White matter and schizophrenia: A meta-analysis of voxel-based morphometry and diffusion tensor imaging studies. Psychiatry Res. - Neuroimaging 270, 8-21 (2017)

31. Bora, E. et al. Neuroanatomical abnormalities in schizophrenia: A multimodal voxelwise meta-analysis and meta-regression analysis. Schizophrenia Research 127, 46-57 (2011).

32. De Picker, L. J., Morrens, M., Chance, S. A. \& Boche, D. Microglia and brain plasticity in acute psychosis and schizophrenia illness course: A meta-review. Frontiers in Psychiatry 8, (2017).

33. Mesholam-Gately, R. I., Giuliano, A. J., Goff, K. P., Faraone, S. V. \& Seidman, L. J. Neurocognition in First-Episode Schizophrenia: A Meta-Analytic Review. Neuropsychology 23, 315-336 (2009).

34. Capuzzi, E., Bartoli, F., Crocamo, C., Clerici, M. \& Carrà, G. Acute variations of cytokine levels after antipsychotic treatment in drug-naïve subjects with a first-episode psychosis: A meta-analysis. Neuroscience and Biobehavioral Reviews 77, 122-128 (2017). 
35. Sun, H. et al. Two patterns of white matter abnormalities in medication-naive patients with first-episode schizophrenia revealed by diffusion tensor imaging and cluster analysis. JAMA Psychiatry 72, 678-686 (2015).

36. Sanz, J., Reig, S., Benito, C. \& Leal, I. Dorsolateral prefrontal N -acetyl-aspartate concentration in male patients with chronic schizophrenia and with chronic bipolar disorder. 22, 505-512 (2007).

37. Dong, D. et al. Common and diagnosis-specific fractional anisotropy of white matter in schizophrenia, bipolar disorder, and major depressive disorder: Evidence from comparative voxel-based meta-analysis. Schizophrenia Research 193, 456-458 (2018).

38. Goldsmith, D. R., Rapaport, M. H. \& Miller, B. J. A meta-analysis of blood cytokine network alterations in psychiatric patients: Comparisons between schizophrenia, bipolar disorder and depression. in Molecular Psychiatry 21, 1696-1709 (2016).

39. Cauda, F. et al. Are schizophrenia, autistic, and obsessive spectrum disorders dissociable on the basis of neuroimaging morphological findings?: A voxel-based meta-analysis. Autism Res. 10, 1079-1095 (2017).

40. Escudero, I. \& Johnstone, M. Genetics of Schizophrenia. Curr. Psychiatry Rep. (2014).

41. Prata, D. P. et al. Opposite effects of catechol-O-methyltransferase Val158Met on cortical function in healthy subjects and patients with schizophrenia. Biol. Psychiatry 65, 473-80 (2009).

42. Bian, Q. et al. The effect of atypical antipsychotics, perospirone, ziprasidone and quetiapine on microglial activation induced by interferon- $\psi$. Prog. NeuroPsychopharmacology Biol. Psychiatry 32, 42-48 (2008).

43. Rzhetsky, A., Wajngurt, D., Park, N. \& Zheng, T. Probing genetic overlap among complex human phenotypes. Proc. Natl. Acad. Sci. 104, 11694-11699 (2007).

44. Curtis, C. et al. The genomic and transcriptomic architecture of 2,000 breast tumours reveals novel subgroups. Nature 486, 346-352 (2012).

45. Walker, E., Kestler, L., Bollini, A. \& Hochman, K. M. Schizophrenia: Etiology and Course. Annu. Rev. Psychol. 55, 401-430 (2004).

46. Lichtenstein, P. et al. Common genetic determinants of schizophrenia and bipolar disorder in Swedish families: a population-based study. Lancet 373, 234-239 (2009).

47. Wray, N. R. \& Gottesman, I. I. Using summary data from the Danish National Registers to estimate heritabilities for schizophrenia, bipolar disorder, and major depressive disorder. Front. Genet. 3, (2012).

48. Ripke, S. et al. Genome-wide association analysis identifies 13 new risk loci for schizophrenia. Nat. ... 45, 1-26 (2013).

49. Sullivan, P. F., Kendler, K. S. \& Neale, M. C. Schizophrenia as a Complex Trait: Evidence from a Meta-analysis of Twin Studies. Arch. Gen. Psychiatry 60, 1187-1192 (2003).

50. Lee, S. H. et al. Estimating the proportion of variation in susceptibility to schizophrenia captured by common SNPs. Nat. Genet. 44, 247-250 (2012).

51. Réthelyi, J. M., Benkovits, J. \& Bitter, I. Interplay between childhood trauma and BDNF val66met variants on blood BDNF mRNA levels and on hippocampus subfields volumes in schizophrenia spectrum and bipolar disorders. Neuroscience and Biobehavioral Reviews 37, 2424-2437 (2013).

52. Foley, C., Corvin, A. \& Nakagome, S. Genetics of Schizophrenia: Ready to Translate? 
Current Psychiatry Reports 19, (2017).

53. Brookes, A. J. The essence of SNPs. Gene 234, 177-186 (1999).

54. Chen, J., Cao, F., Liu, L., Wang, L. \& Chen, X. Genetic studies of schizophrenia: An update. Neuroscience Bulletin 31, 87-98 (2015).

55. Rutkowski, T. P. et al. Unraveling the genetic architecture of copy number variants associated with schizophrenia and other neuropsychiatric disorders. Journal of Neuroscience Research 95, 1144-1160 (2017).

56. Zhuo, C., Hou, W., Lin, C., Hu, L. \& Li, J. Potential Value of Genomic Copy Number Variations in Schizophrenia. Front. Mol. Neurosci. 10, (2017).

57. International Schizophrenia Consortium, T. I. S. Rare chromosomal deletions and duplications increase risk of schizophrenia. Nature 455, 237-41 (2008).

58. Arnedo, J. et al. Uncovering the hidden risk architecture of the schizophrenias: Confirmation in three independent genome-wide association studies. Am. J. Psychiatry 172, 139-153 (2015).

59. Anttila, V. et al. Analysis of shared heritability in common disorders of the brain. Science (80-. ). 360, eaap8757 (2018).

60. Ferrarelli, F. Endophenotypes and Biological Markers of Schizophrenia: From Biological Signs of Illness to Novel Treatment Targets. Curr. Pharm. Des. 19, 6462-6479 (2013).

61. Lett, T. A. et al. The genome-wide supported microRNA-137 variant predicts phenotypic heterogeneity within schizophrenia. Mol. Psychiatry 18, 443-450 (2013).

62. Pitcher, G. M. et al. Schizophrenia susceptibility pathway neuregulin 1-ErbB4 suppresses Src upregulation of NMDA receptors. Nat. Med. 17, 470-478 (2011).

63. Kempton, M. J., Stahl, D., Williams, S. C. R. \& DeLisi, L. E. Progressive lateral ventricular enlargement in schizophrenia: A meta-analysis of longitudinal MRI studies. Schizophr. Res. 120, 54-62 (2010).

64. Ellison-Wright, I. \& Bullmore, E. Meta-analysis of diffusion tensor imaging studies in schizophrenia. Schizophr. Res. 108, 3-10 (2009).

65. Harrison, P. J. \& Owen, M. J. Genes for schizophrenia? Recent findings and their pathophysiological implications. Lancet 361, 417-419 (2003).

66. Wheeler, A. L. \& Voineskos, A. N. A review of structural neuroimaging in schizophrenia: from connectivity to connectomics. Front. Hum. Neurosci. 8, (2014).

67. Honea, R., Crow, T. J., Passingham, D. \& Mackay, C. E. Regional deficits in brain volume in schizophrenia: A meta-analysis of voxel-based morphometry studies. American Journal of Psychiatry 162, 2233-2245 (2005).

68. Andreasen, N. C. The lifetime trajectory of schizophrenia and the concept of neurodevelopment. Dialogues Clin. Neurosci. 12, 409-415 (2010).

69. Paper, O. et al. Dorsolateral prefrontal and superior temporal volume deficits in firstepisode psychoses that evolve into schizophrenia. Eur. Arch. Psychiatry Clin. Neurosci. 256, 106-111 (2006).

70. Vita, A., De Peri, L., Deste, G., Barlati, S. \& Sacchetti, E. The Effect of Antipsychotic Treatment on Cortical Gray Matter Changes in Schizophrenia: Does the Class Matter? A Meta-analysis and Meta-regression of Longitudinal Magnetic Resonance Imaging Studies. Biol. Psychiatry 78, 403-412 (2015). 
71. Vita, A., De Peri, L., Deste, G. \& Sacchetti, E. Progressive loss of cortical gray matter in schizophrenia: A meta-analysis and meta-regression of longitudinal MRI studies. Translational Psychiatry 2, (2012).

72. Fraguas, D., Díaz-Caneja, C. M., Pina-Camacho, L., Janssen, J. \& Arango, C. Progressive brain changes in children and adolescents with early-onset psychosis: A meta-analysis of longitudinal MRI studies. Schizophr. Res. 173, 132-139 (2016).

73. Selemon, L. D. \& Goldman-Rakic, P. S. The reduced neuropil hypothesis: a circuit based model of schizophrenia. Biol. Psychiatry 45, 17-25 (1999).

74. Sekar, A. et al. Schizophrenia risk from complex variation of complement component 4. Nature 530, 177-183 (2016).

75. Glausier, J. R. \& Lewis, D. A. Dendritic spine pathology in schizophrenia. Neuroscience 251, 90-107 (2013).

76. Sweet, R. A. Mapping synaptic pathology within cerebral cortical circuits in subjects with schizophrenia. Front. Hum. Neurosci. (2010).

77. Flores, G., Morales-Medina, J. C. \& Diaz, A. Neuronal and brain morphological changes in animal models of schizophrenia. Behavioural Brain Research 301, 190-203 (2016).

78. Osimo, E. F., Beck, K., Reis Marques, T. \& Howes, O. D. Synaptic loss in schizophrenia: a meta-analysis and systematic review of synaptic protein and mRNA measures. Molecular Psychiatry 1-13 (2018).

79. Wang, C., Aleksic, B. \& Ozaki, N. Glia-related genes and their contribution to schizophrenia. Psychiatry Clin. Neurosci. 69, 448-461 (2015).

80. Nenadic, I. et al. ZNF804A genetic variation ( $r$ 1344706) affects brain grey but not white matter in schizophrenia and healthy subjects. Psychol. Med. 45, 143-152 (2015).

81. Nenadic, I., Yotter, R. A., Sauer, H. \& Gaser, C. Patterns of cortical thinning in different subgroups of schizophrenia. Br. J. Psychiatry 206, 479-483 (2015).

82. Altamura, A. C. et al. Gray matter volumes may predict the clinical response to paliperidone palmitate long-acting in acute psychosis: A pilot longitudinal neuroimaging study. Psychiatry Res. - Neuroimaging 261, 80-84 (2017).

83. Koutsouleris, N. et al. Predicting Response to Repetitive Transcranial Magnetic Stimulation in Patients With Schizophrenia Using Structural Magnetic Resonance Imaging: A Multisite Machine Learning Analysis. Schizophr. Bull. (2017).

84. Goodkind, M. et al. Identification of a common neurobiological substrate for mental Illness. JAMA Psychiatry 72, 305-315 (2015).

85. Varela, F., Lachaux, J. P., Rodriguez, E. \& Martinerie, J. The brainweb: phase synchronization and large-scale integration. Nat. Rev. Neurosci. 2, 229-39 (2001).

86. Dehaene, S. \& Changeux, J. P. Experimental and Theoretical Approaches to Conscious Processing. Neuron 70, 200-227 (2011).

87. Sporns, O. Networks of the Brain. MIT Press. Cambridge, MA (2009).

88. Friston, K. J. Functional and effective connectivity in neuroimaging: A synthesis. Hum. Brain Mapp. 2, 56-78 (1994).

89. Friston, K. J. The disconnection hypothesis. Schizophr. Res. 30, 115-25 (1998).

90. Skudlarski, P. et al. Brain Connectivity Is Not Only Lower but Different in Schizophrenia: A Combined Anatomical and Functional Approach. Biol. Psychiatry 68, 61-69 (2010). 
91. Kubicki, M. et al. A review of diffusion tensor imaging studies in schizophrenia. $J$. Psychiatr. Res. 41, 15-30 (2007).

92. Tamnes, C. K. \& Agartz, I. White Matter Microstructure in Early-Onset Schizophrenia: A Systematic Review of Diffusion Tensor Imaging Studies. Journal of the American Academy of Child and Adolescent Psychiatry 55, 269-279 (2016).

93. Yao, L. et al. White matter deficits in first episode schizophrenia: An activation likelihood estimation meta-analysis. Prog. Neuro-Psychopharmacology Biol. Psychiatry 45, 100-106 (2013).

94. Dong, D., Wang, Y., Chang, X., Luo, C. \& Yao, D. Dysfunction of Large-Scale Brain Networks in Schizophrenia: A Meta-analysis of Resting-State Functional Connectivity. Schizophr. Bull. (2017).

95. Van Den Heuvel, M. P. \& Fornito, A. Brain networks in schizophrenia. Neuropsychology Review 24, 32-48 (2014).

96. Fornito, A., Zalesky, A., Pantelis, C. \& Bullmore, E. T. Schizophrenia, neuroimaging and connectomics. Neurolmage 62, 2296-2314 (2012).

97. Meda, S. a et al. Multivariate analysis reveals genetic associations of the resting default mode network in psychotic bipolar disorder and schizophrenia. Proc. Natl. Acad. Sci. U. S. A. 111, E2066-75 (2014).

98. Thalbourne, M. A., Houran, J., Alias, A. G. \& Brugger, P. Transliminality, brain function, and synesthesia. J. Nerv. Ment. Dis. 189, 190-192 (2001).

99. Salvador, R. et al. Overall brain connectivity maps show cortico-subcortical abnormalities in schizophrenia. Hum. Brain Mapp. 31, 2003-2014 (2010).

100. Guerrero-Pedraza, A. et al. First-episode psychosis is characterized by failure of deactivation but not by hypo- or hyperfrontality. Psychol. Med. 42, 73-84 (2012).

101. Whitfield-Gabrieli, S. et al. Hyperactivity and hyperconnectivity of the default network in schizophrenia and in first-degree relatives of persons with schizophrenia. Proc. Natl. Acad. Sci. 106, 1279-1284 (2009).

102. Li, T. et al. Brain-Wide Analysis of Functional Connectivity in First-Episode and Chronic Stages of Schizophrenia. Schizophr. Bull. 43, 436-448 (2017).

103. Guo, Q. et al. Both volumetry and functional connectivity of Heschl's gyrus are associated with auditory P300 in first episode schizophrenia. Schizophr. Res. 160, 57-66 (2014).

104. Wolf, R. C. et al. Temporally anticorrelated brain networks during working memory performance reveal aberrant prefrontal and hippocampal connectivity in patients with schizophrenia. Prog. Neuro-Psychopharmacology Biol. Psychiatry 33, 1464-1473 (2009).

105. Fujimoto, T. et al. Dysfunctional Cortical Connectivity During the Auditory Oddball Task in Patients with Schizophrenia. Open Neuroimag. J. 7, 15-26 (2013).

106. Dong, D. et al. Shared abnormality of white matter integrity in schizophrenia and bipolar disorder: A comparative voxel-based meta-analysis. Schizophr. Res. 185, 41-50 (2017).

107. Baribeau, D. A. \& Anagnostou, E. A comparison of neuroimaging findings in childhood onset schizophrenia and autism spectrum disorder: A review of the literature. Frontiers in Psychiatry 4, (2013).

108. Wei, Y. et al. Local functional connectivity alterations in schizophrenia, bipolar disorder, and major depressive disorder. J. Affect. Disord. 236, 266-273 (2018).

109. Bullmore, E. \& Sporns, O. Complex brain networks: graph theoretical analysis of 
structural and functional systems. Nat. Publ. Gr. 10, 186-198 (2009).

110. Friston, K. J. Functional and Effective Connectivity: A Review. Brain Connect. 1, 13-36 (2011).

111. Yu, S., Huang, D., Singer, W. \& Nikolić, D. A small world of neuronal synchrony. Cereb. Cortex 18, 2891-2901 (2008).

112. Sporns, O. The human connectome: a complex network. Hum. Connect. 1224, 109-125 (2011).

113. Gong, G. et al. Mapping anatomical connectivity patterns of human cerebral cortex using in vivo diffusion tensor imaging tractography. Cereb. Cortex 19, 524-536 (2009).

114. Bassett, D. S. \& Bullmore, E. T. Human brain networks in health and disease. Current Opinion in Neurology 22, 340-347 (2009).

115. Shim, M., Kim, D. W., Lee, S. H. \& Im, C. H. Disruptions in small-world cortical functional connectivity network during an auditory oddball paradigm task in patients with schizophrenia. Schizophr. Res. 156, 197-203 (2014).

116. Borsboom, D., Cramer, A. O. J., Schmittmann, V. D., Epskamp, S. \& Waldorp, L. J. The Small World of Psychopathology. PLoS One 6, (2011).

117. Gomez-Pilar, J. et al. Functional EEG network analysis in schizophrenia: Evidence of larger segregation and deficit of modulation. Prog. Neuro-Psychopharmacology Biol. Psychiatry 76, 116-123 (2017).

118. Griffa, A. et al. Characterizing the connectome in schizophrenia with diffusion spectrum imaging. Hum. Brain Mapp. 36, 354-366 (2015).

119. Zalesky, A. et al. Disrupted axonal fiber connectivity in schizophrenia. Biol. Psychiatry 69, 80-89 (2011).

120. Gomez-Pilar, J. et al. Relations between structural and EEG-based graph metrics in healthy controls and schizophrenia patients. Hum. Brain Mapp. 1-14 (2018).

121. Crossley, N. a. et al. The hubs of the human connectome are generally implicated in the anatomy of brain disorders. Brain 137, 2382-2395 (2014).

122. Van Den Heuvel, M. P. et al. Abnormal rich club organization and functional brain dynamics in schizophrenia. JAMA Psychiatry 70, 783-792 (2013).

123. Rubinov, M. et al. Small-world properties of nonlinear brain activity in schizophrenia. Hum. Brain Mapp. 30, 403-416 (2009).

124. Kambeitz, J. et al. Aberrant Functional Whole-Brain Network Architecture in Patients with Schizophrenia: A Meta-analysis. Schizophr. Bull. 42, S13-S21 (2016).

125. Micheloyannis, S. et al. Small-world networks and disturbed functional connectivity in schizophrenia. Schizophr. Res. 87, 60-66 (2006).

126. Micheloyannis, S. et al. Using graph theoretical analysis of multi channel EEG to evaluate the neural efficiency hypothesis. Neurosci. Lett. 402, 273-277 (2006).

127. Jhung, K. et al. Small-world networks in individuals at ultra-high risk for psychosis and first-episode schizophrenia during a working memory task. Neurosci. Lett. 535, 35-39 (2013).

128. Pachou, E. et al. Working memory in schizophrenia: An EEG study using power spectrum and coherence analysis to estimate cortical activation and network behavior. Brain Topogr. 21, 128-137 (2008). 
129. Crossley, N. A. et al. Altered hub functioning and compensatory activations in the connectome: A meta- Analysis of functional neuroimaging studies in schizophrenia. Schizophr. Bull. 42, 434-442 (2016).

130. Fitzsimmons, J., Kubicki, M. \& Shenton, M. E. Review of functional and anatomical brain connectivity findings in schizophrenia. Current Opinion in Psychiatry 26, 172-187 (2013).

131. Liu, H., Fan, G., Xu, K. \& Wang, F. Changes in cerebellar functional connectivity and anatomical connectivity in schizophrenia: A combined resting-state functional MRI and diffusion tensor imaging study. J. Magn. Reson. Imaging 34, 1430-1438 (2011).

132. Koch, K. et al. Neural activation and radial diffusivity in schizophrenia: Combined fMRI and diffusion tensor imaging study. Br. J. Psychiatry 198, 223-229 (2011).

133. Cabral, J. et al. Structural connectivity in schizophrenia and its impact on the dynamics of spontaneous functional networks. Chaos 23, (2013).

134. Gao, X. et al. Association between structural and functional brain alterations in drug-free patients with schizophrenia: A multimodal meta-analysis. J. Psychiatry Neurosci. 43, 131142 (2018).

135. Reis Marques, T. et al. White matter integrity as a predictor of response to treatment in first episode psychosis. Brain 137, 172-82 (2014).

136. Alonso-Solís, A. et al. Resting-state functional connectivity alterations in the default network of schizophrenia patients with persistent auditory verbal hallucinations. Schizophr. Res. 161, 261-268 (2015).

137. Uddin, L. Q. Chapter 3 - Functions of the Salience Network. in Salience Network of the Human Brain 11-16 (2017).

138. White, T. P. et al. Dysfunctional Striatal Systems in Treatment-Resistant Schizophrenia. Neuropsychopharmacology 41, 1274-1285 (2016).

139. Carpenter, W. T., Heinrichs, D. W. \& Wagman, A. M. I. Deficit and nondeficit forms of schizophrenia: The concept. Am. J. Psychiatry 145, 578-583 (1988).

140. Yu, M. et al. Convergence and Divergence of Brain Network Dysfunction in Deficit and Non-deficit Schizophrenia. Schizophr. Bull. 43, 1315-1328 (2017).

141. Sarpal, D. K. et al. Antipsychotic treatment and functional connectivity of the striatum in first-episode schizophrenia. JAMA Psychiatry 72, 5-13 (2015).

142. Lui, S. et al. Short-term effects of antipsychotic treatment on cerebral function in drugnaive first-episode schizophrenia revealed by 'resting state' functional magnetic resonance imaging. Arch. Gen. Psychiatry 67, 783-792 (2010).

143. Hutcheson, N. L. et al. Effective connectivity during episodic memory retrieval in schizophrenia participants before and after antipsychotic medication. Hum. Brain Mapp. 36, 1442-1457 (2015).

144. Uhlhaas, P. J. \& Singer, W. Abnormal neural oscillations and synchrony in schizophrenia. Nature Reviews Neuroscience 11, 100-113 (2010).

145. Schwartz, T. L., Sachdeva, S. \& Stahl, S. M. Glutamate neurocircuitry: Theoretical underpinnings in: Schizophrenia. Front. Pharmacol. 3 NOV, 1-11 (2012).

146. Howes, O. D., McCutcheon, R., Owen, M. J. \& Murray, R. M. The Role of Genes, Stress, and Dopamine in the Development of Schizophrenia. Biological Psychiatry 81, 9-20 (2017).

147. Nakazawa, K. et al. GABAergic interneuron origin of schizophrenia pathophysiology. 
Neuropharmacology 62, 1574-83 (2012).

148. Meltzer, H. Y. \& Stahl, S. M. The dopamine hypothesis of schizophrenia: a review. Schizophrenia Bulletin 2, 19-76 (1976).

149. Maia, T. V. \& Frank, M. J. An Integrative Perspective on the Role of Dopamine in Schizophrenia. Biological Psychiatry 81, 52-66 (2017).

150. Nakata, Y., Kanahara, N. \& Iyo, M. Dopamine supersensitivity psychosis in schizophrenia: Concepts and implications in clinical practice. Journal of Psychopharmacology 31, 15111518 (2017).

151. Howes, O. D. \& Kapur, S. A neurobiological hypothesis for the classification of schizophrenia: Type a (hyperdopaminergic) and type b (normodopaminergic). British Journal of Psychiatry 205, 1-3 (2014).

152. Pavăl, D. A Dopamine Hypothesis of Autism Spectrum Disorder. Developmental Neuroscience 39, 355-360 (2017).

153. Kantrowitz, J. T. \& Javitt, D. C. N-methyl-d-aspartate (NMDA) receptor dysfunction or dysregulation: The final common pathway on the road to schizophrenia? Brain Res. Bull. 83, 108-121 (2010).

154. Halene, T. B. et al. Assessment of NMDA receptor NR1 subunit hypofunction in mice as a model for schizophrenia. Genes, Brain Behav. 8, 661-675 (2009).

155. Miyamoto, Y. et al. Hyperfunction of dopaminergic and serotonergic neuronal systems in mice lacking the NMDA receptor epsilon1 subunit. J. Neurosci. 21, 750-7 (2001).

156. Beck, K., Javitt, D. C. \& Howes, O. D. Targeting glutamate to treat schizophrenia: lessons from recent clinical studies. Psychopharmacology 233, 2425-2428 (2016).

157. Gutiérrez-Fernández, A. et al. Novel association of Neuregulin 1 gene with bipolar disorder but not with schizophrenia. Schizophr. Res. 159, 552-553 (2014).

158. Díez, Á., Suazo, V., Casado, P., Martín-Loeches, M. \& Molina, V. Spatial distribution and cognitive correlates of gamma noise power in schizophrenia. Psychol. Med. 1-11 (2012).

159. Suazo, V. et al. Progress in Neuro-Psychopharmacology \& Biological Psychiatry Elevated noise power in gamma band related to negative symptoms and memory de fi cit in schizophrenia. Prog. Neuropsychopharmacol. Biol. Psychiatry (2012).

160. Dawson, N., McDonald, M., Higham, D. J., Morris, B. J. \& Pratt, J. A. Subanesthetic ketamine treatment promotes abnormal interactions between neural subsystems and alters the properties of functional brain networks. Neuropsychopharmacology 39, 17861798 (2014).

161. Driesen, N. R. et al. Relationship of resting brain hyperconnectivity and schizophrenialike symptoms produced by the NMDA receptor antagonist ketamine in humans. Mol. Psychiatry 18, 1199-1204 (2013).

162. Neddens, J. et al. Conserved interneuron-specific ErbB4 expression in frontal cortex of rodents, monkeys, and humans: implications for schizophrenia. Biol. Psychiatry 70, 63645 (2011).

163. Lewis, D. A., Hashimoto, T. \& Volk, D. W. Cortical inhibitory neurons and schizophrenia. Nature Reviews Neuroscience 6, 312-324 (2005).

164. Egerton, A. et al. Anterior cingulate glutamate levels related to clinical status following treatment in first-episode schizophrenia. Neuropsychopharmacology 37, 2515-21 (2012). 
165. Mouchlianitis, E. et al. Treatment-Resistant Schizophrenia Patients Show Elevated Anterior Cingulate Cortex Glutamate Compared to Treatment-Responsive. Schizophr. Bull. 42, 744-752 (2016).

166. Oda, Y. et al. Alterations in glutamatergic signaling in the brain of dopamine supersensitivity psychosis and non-supersensitivity psychosis model rats. Psychopharmacology (Berl). 234, 3027-3036 (2017).

167. Benros, M. E., Eaton, W. W. \& Mortensen, P. B. The epidemiologic evidence linking autoimmune diseases and psychosis. Biological Psychiatry 75, 300-306 (2014).

168. Oldham, M. Autoimmune Encephalopathy for Psychiatrists: When to Suspect Autoimmunity and What to Do Next. Psychosomatics 58, 228-244 (2017).

169. Khandaker, G. M., Zimbron, J., Lewis, G. \& Jones, P. B. Prenatal maternal infection, neurodevelopment and adult schizophrenia: A systematic review of population-based studies. Psychol. Med. 43, 239-257 (2013).

170. Liang, W. \& Chikritzhs, T. Early childhood infections and risk of schizophrenia. Psychiatry Res. 200, 214-217 (2012).

171. Miller, B. J. \& Goldsmith, D. R. Towards an Immunophenotype of Schizophrenia: Progress, Potential Mechanisms, and Future Directions. Neuropsychopharmacology 42, 299-317 (2017).

172. Fillman, S. G. et al. Increased inflammatory markers identified in the dorsolateral prefrontal cortex of individuals with schizophrenia. Mol. Psychiatry 18, 206-214 (2013).

173. Trépanier, M. O., Hopperton, K. E., Mizrahi, R., Mechawar, N. \& Bazinet, R. P. Postmortem evidence of cerebral inflammation in schizophrenia: A systematic review. Molecular Psychiatry 21, 1009-1026 (2016).

174. Miller, B. J., Buckley, P., Seabolt, W., Mellor, A. \& Kirkpatrick, B. Meta-analysis of cytokine alterations in schizophrenia: Clinical status and antipsychotic effects. Biol. Psychiatry 70, 663-671 (2011).

175. Na, K.-S., Jung, H.-Y. \& Kim, Y.-K. The role of pro-inflammatory cytokines in the neuroinflammation and neurogenesis of schizophrenia. Prog. NeuroPsychopharmacology Biol. Psychiatry 48, 277-286 (2014).

176. Najjar, S. \& Pearlman, D. M. Neuroinflammation and white matter pathology in schizophrenia: Systematic review. Schizophrenia Research 161, 102-112 (2015).

177. Anderson, G. \& Maes, M. Schizophrenia: Linking prenatal infection to cytokines, the tryptophan catabolite (TRYCAT) pathway, NMDA receptor hypofunction, neurodevelopment and neuroprogression. Prog. Neuro-Psychopharmacology Biol. Psychiatry 42, 5-19 (2013).

178. Seki, Y. et al. Pretreatment of aripiprazole and minocycline, but not haloperidol, suppresses oligodendrocyte damage from interferon- $\gamma$-stimulated microglia in co-culture model. Schizophr. Res. 151, 20-28 (2013).

179. Fillman, S. G., Sinclair, D., Fung, S. J., Webster, M. J. \& Shannon Weickert, C. Markers of inflammation and stress distinguish subsets of individuals with schizophrenia and bipolar disorder. Transl. Psychiatry 4, (2014).

180. Catts, V. S., Wong, J., Fillman, S. G., Fung, S. J. \& Shannon Weickert, C. Increased expression of astrocyte markers in schizophrenia: Association with neuroinflammation. Aust. N. Z. J. Psychiatry 48, 722-734 (2014). 
181. Zhang, Y. et al. Cortical grey matter volume reduction in people with schizophrenia is associated with neuro-inflammation. Transl. Psychiatry 6, (2016).

182. Fillman, S. G. et al. Elevated peripheral cytokines characterize a subgroup of people with schizophrenia displaying poor verbal fluency and reduced Broca's area volume. Mol. Psychiatry 21, 1090-1098 (2016).

183. Schwarz, E. et al. Identification of subgroups of Schizophrenia patients with changes in either immune or growth factor and hormonal pathways. Schizophr. Bull. 40, 787-795 (2014).

184. Millan, M. J. et al. Deconstructing vulnerability for psychosis: Meta-analysis of environmental risk factors for psychosis in subjects at ultra high-risk. European Psychiatry 40, 65-75 (2017).

185. Van Os, J., Kenis, G. \& Rutten, B. P. F. The environment and schizophrenia. Nature 468, 203-212 (2010).

186. Morgan, C. \& Fisher, H. Environment and schizophrenia: Environmental factors in schizophrenia: Childhood trauma - A critical review. Schizophrenia Bulletin 33, 3-10 (2007).

187. Brown, A. S. The environment and susceptibility to schizophrenia. Progress in Neurobiology 93, 23-58 (2011).

188. Cantor-Graae, E. \& Selten, J. P. Schizophrenia and migration: A meta-analysis and review. American Journal of Psychiatry 162, 12-24 (2005).

189. Morgan, C., Charalambides, M., Hutchinson, G. \& Murray, R. M. Migration, ethnicity, and psychosis: Toward a sociodevelopmental model. Schizophr. Bull. 36, 655-664 (2010).

190. Vassos, E., Pedersen, C. B., Murray, R. M., Collier, D. A. \& Lewis, C. M. Meta-analysis of the association of urbanicity with schizophrenia. Schizophr. Bull. 38, 1118-1123 (2012).

191. Kelly, B. D. et al. Schizophrenia and the city: A review of literature and prospective study of psychosis and urbanicity in Ireland. Schizophr. Res. 116, 75-89 (2010).

192. Zammit, S. et al. Individuals, schools, and neighborhood: A multilevel longitudinal study of variation in incidence of psychotic disorders. Arch. Gen. Psychiatry 67, 914-922 (2010).

193. Besteher, B., Gaser, C., Spalthoff, R. \& Nenadić, I. Associations between urban upbringing and cortical thickness and gyrification. J. Psychiatr. Res. 95, 114-120 (2017).

194. Bonoldi, I. et al. Prevalence of self-reported childhood abuse in psychosis: A metaanalysis of retrospective studies. Psychiatry Res. 210, 8-15 (2013).

195. Larkin, W. \& Read, J. Childhood trauma and psychosis: evidence, pathways, and implications. J. Postgrad. Med. 54, 287-93 (2008).

196. Varese, F. et al. Childhood adversities increase the risk of psychosis: A meta-analysis of patient-control, prospective-and cross-sectional cohort studies. Schizophr. Bull. 38, 661671 (2012).

197. Baudin, G. et al. Differential effects of childhood trauma and cannabis use disorders in patients suffering from schizophrenia. Schizophr. Res. 175, 161-167 (2016).

198. Bailey, T. et al. Childhood Trauma Is Associated With Severity of Hallucinations and Delusions in Psychotic Disorders: A Systematic Review and Meta-Analysis. Schizophr. Bull. (2018).

199. Hernaus, D. et al. Brain-derived neurotrophic factor/FK506-binding protein 5 genotype by childhood trauma interactions do not impact on hippocampal volume and cognitive 
performance. PLoS One 9, (2014).

200. Casadio, P., Fernandes, C., Murray, R. M. \& Di Forti, M. Cannabis use in young people: The risk for schizophrenia. Neuroscience and Biobehavioral Reviews 35, 1779-1787 (2011).

201. Arseneault, L. et al. Cannabis use in adolescence and risk for adult psychosis: longitudinal prospective study. BMJ 325, 1212-1213 (2002).

202. Ferdinand, R. F. et al. Cannabis use predicts future psychotic symptoms, and vice versa. Addiction 100, 612-618 (2005).

203. Wade, D. et al. Substance misuse in first-episode psychosis: 15-month prospective follow-up study. Br J Psychiatry 189, 229-234 (2006).

204. Seddon, J. L. et al. Cannabis use is associated with increased psychotic symptoms and poorer psychosocial functioning in first-episode psychosis: A report from the UK National EDEN study. Schizophr. Bull. 42, 619-625 (2016).

205. Mané, A. et al. Relationship between cannabis and psychosis: Reasons for use and associated clinical variables. Psychiatry Res. 229, 70-74 (2015).

206. Batalla, A. et al. The Influence of DAT1, COMT, and BDNF Genetic Polymorphisms on Total and Subregional Hippocampal Volumes in Early Onset Heavy Cannabis Users. Cannabis Cannabinoid Res. 3, 1-10 (2018).

207. Caspi, A. et al. Moderation of the effect of adolescent-onset cannabis use on adult psychosis by a functional polymorphism in the catechol-O-methyltransferase gene: Longitudinal evidence of a gene X environment interaction. Biol. Psychiatry 57, 11171127 (2005).

208. Estrada, G. et al. Cannabis use and age at onset of psychosis: Further evidence of interaction with COMT Val158Met polymorphism. Acta Psychiatr. Scand. 123, 485-492 (2011).

209. Misiak, B. et al. Interactions Between Variation in Candidate Genes and Environmental Factors in the Etiology of Schizophrenia and Bipolar Disorder: a Systematic Review. Molecular Neurobiology 1-26 (2017).

210. Vaessen, T. S. J. et al. The interaction between cannabis use and the Val158Met polymorphism of the COMT gene in psychosis: A transdiagnostic meta \pm analysis. PLOS One 13, (2018).

211. Zammit, S., Owen, M. J., Evans, J., Heron, J. \& Lewis, G. Cannabis, COMT and psychotic experiences. Br. J. Psychiatry 199, 380-385 (2011).

212. Gracious, B. L., Finucane, T. L., Friedman-Campbell, M., Messing, S. \& Parkhurst, M. N. Vitamin $D$ deficiency and psychotic features in mentally ill adolescents: A cross-sectional study. BMC Psychiatry 12, (2012).

213. Hedelin, M. et al. Dietary intake of fish, omega-3, omega- 6 polyunsaturated fatty acids and vitamin $D$ and the prevalence of psychotic-like symptoms in a cohort of 33,000 women from the general population. BMC Psychiatry 10, 38 (2010).

214. Bonnot, O. et al. Children and Adolescents with Severe Mental Illness Need Vitamin D Supplementation Regardless of Disease or Treatment. J. Child Adolesc. Psychopharmacol. 21, 157-161 (2011).

215. Amminger, G. P. et al. Long-chain omega-3 fatty acids for indicated prevention of psychotic disorders: a randomized, placebo-controlled trial. Arch. Gen. Psychiatry 67, 
146-54 (2010).

216. Cariaga-Martinez, A. \& Paz, R. A. Rethinking the epigenetic framework to unravel the molecular pathology of schizophrenia. International Journal of Molecular Sciences 18, (2017).

217. Shorter, K. R. \& Miller, B. H. Epigenetic mechanisms in schizophrenia. Progress in Biophysics and Molecular Biology 118, 1-7 (2015).

218. Portela, A. \& Esteller, M. Epigenetic modifications and human disease. Nature Biotechnology 28, 1057-1068 (2010).

219. Uher, R. Gene-environment interactions in severe mental illness. Front. Psychiatry 5, 19 (2014).

220. Insel, T. et al. Research Domain Criteria ( RDoC ): Toward a. Am. J. Psychiatry 167, 748751 (2010).

221. Insel, T. R. The nimh research domain criteria ( $\mathrm{rdoc}$ ) project: Precision medicine for psychiatry. American Journal of Psychiatry 171, 395-397 (2014).

222. Papiol, S. et al. Ventricular enlargement in schizophrenia is associated with a genetic polymorphism at the interleukin-1 receptor antagonist gene. Neuroimage 27, 1002-1006 (2005).

223. Crespo-Facorro, B. et al. Global and regional cortical thinning in first-episode psychosis patients: Relationships with clinical and cognitive features. Psychol. Med. 41, 1449-1460 (2011).

224. van Haren, N. E. M. et al. Changes in cortical thickness during the course of illness in schizophrenia. Arch. Gen. Psychiatry 68, 871-880 (2011).

225. Venkatasubramanian, G., Jayakumar, P. N., Gangadhar, B. N. \& Keshavan, M. S. Automated MRI parcellation study of regional volume and thickness of prefrontal cortex (PFC) in antipsychotic-naïve schizophrenia. Acta Psychiatr. Scand. 117, 420-431 (2008).

226. Goldman, A. L. et al. Widespread reductions of cortical thickness in schizophrenia and spectrum disorders and evidence of heritability. Arch. Gen. Psychiatry 66, 467-477 (2009).

227. Blasi, G. et al. Association of GSK-3beta genetic variation with GSK-3beta expression, prefrontal cortical thickness, prefrontal physiology, and schizophrenia. Am. J. Psychiatry 170, 868-876 (2013).

228. Whittle, S. et al. Positive parenting predicts the development of adolescent brain structure: A longitudinal study. Dev. Cogn. Neurosci. 8, 7-17 (2014).

229. Schmitt, J. E. et al. The dynamic role of genetics on cortical patterning during childhood and adolescence. Proc. Natl. Acad. Sci. 111, 6774-6779 (2014).

230. Szeszko, P. R. et al. Magnetic resonance imaging predictors of treatment response in firstepisode schizophrenia. Schizophr. Bull. 38, 569-578 (2012).

231. C.M., C. et al. Association of a risk allele of ANK3 with cognitive performance and cortical thickness in patients with first-episode psychosis. Schizophr. Res. 153, S136-S137 (2014).

232. Ronan, L. et al. Intrinsic Curvature: A marker of millimeter-scale tangential corticocortical connectivity? Int. J. Neural Syst. 21, 351-366 (2011).

233. Falkai, P. et al. Disturbed frontal gyrification within families affected with schizophrenia. J. Psychiatr. Res. 41, 805-813 (2007). 
234. Fornito, A. et al. Surface-based morphometry of the anterior cingulate cortex in first episode schizophrenia. Hum. Brain Mapp. 29, 478-489 (2008).

235. Cachia, A. et al. Cortical folding abnormalities in schizophrenia patients with resistant auditory hallucinations. Neuroimage 39, 927-935 (2008).

236. Harris, J. M. et al. Gyrification in first-episode schizophrenia: A morphometric study. Biol. Psychiatry 55, 141-147 (2004).

237. Piao, X. et al. G protein-coupled receptor-dependent development of human frontal cortex. Sci. (New York, NY) 303, 2033-2036 (2004).

238. White, T., Su, S., Schmidt, M., Kao, C. Y. \& Sapiro, G. The development of gyrification in childhood and adolescence. Brain and Cognition 72, 36-45 (2010).

239. Van Essen, D. C. A tension-based theory of morphogenesis and compact wiring in the central nervous system. Nature 385, 313-318 (1997).

240. Rimol, L. M. et al. Cortical volume, surface area, and thickness in schizophrenia and bipolar disorder. Biol. Psychiatry 71, 552-560 (2012).

241. Molina, V. et al. Anatomical and functional brain variables associated with clozapine response in treatment-resistant schizophrenia. Psychiatry Res. - Neuroimaging 124, 153161 (2003).

242. Molina, V. et al. Cerebral metabolism and risperidone treatment in schizophrenia. Schizophr Res 60, 1-7 (2003).

243. Molina, V., Galindo, G., Cortés, B. \& Hernández, J. A. Voxel-based morphometry comparison between chronic schizophrenia and bipolar patients and healthy controls. Schizophr. Res. 117, 341 (2010).

244. Molina, V. et al. Increase in gray matter and decrease in white matter volumes in the cortex during treatment with atypical neuroleptics in schizophrenia. Schizophr. Res. 80, 61-71 (2005).

245. Kay, S. R., Fiszbein, A. \& Opler, L. A. The positive and negative syndrome scale (PANSS) for schizophrenia. Schizophr. Bull. 13, 261-276 (1987).

246. Molina, V. et al. Dorsolateral prefrontal cortex contribution to abnormalities of the P300 component of the event-related potential in schizophrenia. Psychiatry Res. Neuroimaging 140, 17-26 (2005).

247. Crow, T. J. The Two-syndrome Concept: Origins and Current Status. Schizophr. Bull. 11, 471-488 (1985).

248. White, T. \& Hilgetag, C. C. Gyrification and neural connectivity in schizophrenia. Dev. Psychopathol. 23, 339-352 (2011).

249. Ronan, L. et al. Consistency and interpretation of changes in millimeter-scale cortical intrinsic curvature across three independent datasets in schizophrenia. Neuroimage 63, 611-621 (2012).

250. Deppe, M. et al. Increased cortical curvature reflects white matter atrophy in individual patients with early multiple sclerosis. Neurolmage Clin. 6, 475-487 (2014).

251. Tkachev, D. et al. Oligodendrocyte dysfunction in schizophrenia and bipolar disorder. Lancet 362, 798-805 (2003).

252. Bartlett, E. J. et al. Effects of haloperidol challenge on regional cerebral glucose utilization in normal human subjects. Am. J. Psychiatry 151, 681-686 (1994). 
253. Wolkin, A. et al. Blunted change in cerebral glucose utilization after haloperidol treatment in schizophrenic patients with prominent negative symptoms. Am J Psychiatry 153, 346-354 (1996).

254. Molina Rodríguez, V. et al. SPECT study of regional cerebral perfusion in neurolepticresistant schizophrenic patients who responded or did not respond to clozapine. Am. J. Psychiatry 153, 1343-1346 (1996).

255. Fujimoto, T. et al. Abnormal glucose metabolism in the anterior cingulate cortex in patients with schizophrenia. Psychiatry Res. - Neuroimaging 154, 49-58 (2007).

256. Haznedar, M. M. et al. Cingulate gyrus volume and metabolism in the schizophrenia spectrum. Schizophr. Res. 71, 249-262 (2004).

257. Ho, B.-C., Andreasen, N. C., Ziebell, S., Pierson, R. \& Magnotta, V. Long-term antipsychotic treatment and brain volumes: a longitudinal study of first-episode schizophrenia. Arch. Gen. Psychiatry 68, 128-37 (2011).

258. Wheeler, A. L. et al. Further Neuroimaging Evidence for the Deficit Subtype of Schizophrenia: A Cortical Connectomics Analysis. JAMA psychiatry 72, 1-10 (2015).

259. Lubeiro, A. et al. Identification of two clusters within schizophrenia with different structural, functional and clinical characteristics. Prog. Neuro-Psychopharmacology Biol. Psychiatry 64, 79-86 (2016).

260. Takemura, H., Caiafa, C. F., Wandell, B. A. \& Pestilli, F. Ensemble Tractography. PLoS Comput. Biol. 12, e1004692 (2016).

261. Pettersson-Yeo, W., Allen, P., Benetti, S., McGuire, P. \& Mechelli, A. Dysconnectivity in schizophrenia: Where are we now? Neuroscience and Biobehavioral Reviews 35, 11101124 (2011).

262. Zhou, Y., Fan, L., Qiu, C. \& Jiang, T. Prefrontal cortex and the dysconnectivity hypothesis of schizophrenia. Neuroscience Bulletin 31, 207-219 (2015).

263. Nenadic, I. et al. Prefrontal gyrification in psychotic bipolar I disorder vs. schizophrenia. J. Affect. Disord. 185, 104-107 (2015).

264. Molina, V. et al. Alterations in prefrontal connectivity in schizophrenia assessed using diffusion magnetic resonance imaging. Prog. Neuro-Psychopharmacology Biol. Psychiatry 76, (2017).

265. Fuentes Durá, I., Romero Peris, M., Dasí Vivó, C. \& Ruiz Ruiz, J. C. [Short form of the WAISIII for use with patients with schizophrenia]. Psicothema 22, 202-7 (2010).

266. Segarra, N. et al. Spanish validation of the Brief Assessment in Cognition in Schizophrenia (BACS) in patients with schizophrenia and healthy controls. Eur. Psychiatry 26, 69-73 (2011).

267. Smith, S. M. Fast robust automated brain extraction. Hum. Brain Mapp. 17, 143-155 (2002).

268. Patenaude, B., Smith, S. M., Kennedy, D. N. \& Jenkinson, M. A Bayesian model of shape and appearance for subcortical brain segmentation. Neuroimage 56, 907-922 (2011).

269. Y. Zhang, M. Brady, and S. S. Segmentation of brain MR images through a hidden Markov random field model and the expectation maximization algorithm. IEEE Trans. Med. Imaging, 20, 45.57 (2001).

270. Dhollander, T., Raffelt, D. \& Connelly, A. Unsupervised 3-tissue response function estimation from single-shell or multi-shell diffusion MR data without a co-registered T1 
image. in ISMRM Workshop on Breaking the Barriers of Diffusion MRI 5 (2016).

271. Tournier, J. D., Calamante, F. \& Connelly, A. Robust determination of the fibre orientation distribution in diffusion MRI: Non-negativity constrained super-resolved spherical deconvolution. Neuroimage 35, 1459-1472 (2007).

272. Smith, R. E., Tournier, J. D., Calamante, F. \& Connelly, A. Anatomically-constrained tractography: Improved diffusion MRI streamlines tractography through effective use of anatomical information. Neuroimage 62, 1924-1938 (2012).

273. Salvador, R. et al. Formal characterization and extension of the linearized diffusion tensor model. Hum. Brain Mapp. 24, 144-155 (2005).

274. Schultz, C. C. et al. Increased white matter radial diffusivity is associated with prefrontal cortical folding deficits in schizophrenia. Psychiatry Res. - Neuroimaging 261, 91-95 (2017).

275. Narr, K. L. et al. Abnormal gyral complexity in first-episode schizophrenia. Biol. Psychiatry 55, 859-867 (2004).

276. Schultz, C. C. et al. Increased parahippocampal and lingual gyrification in first-episode schizophrenia. Schizophr. Res. 123, 137-144 (2010).

277. Vogeley, K. et al. Disturbed gyrification of the prefrontal region in male schizophrenic patients: A morphometric postmortem study. Am. J. Psychiatry 157, 34-39 (2000).

278. Wiegand, L. C. et al. An in vivo MRI study of prefrontal cortical complexity in first-episode psychosis. Am. J. Psychiatry 162, 65-70 (2005).

279. Janssen, J. et al. Cortical morphology of adolescents with bipolar disorder and with schizophrenia. Schizophr. Res. 158, 91-99 (2014).

280. Liu, H. et al. Schizophrenic patients and their unaffected siblings share increased restingstate connectivity in the task-negative network but not its anticorrelated task-positive network. Schizophr. Bull. 38, 285-294 (2012).

281. Roalf, D. R. et al. White matter microstructure in schizophrenia: Associations to neurocognition and clinical symptomatology. Schizophr. Res. 161, 42-49 (2015).

282. Díez, Á., Suazo, V., Casado, P., Martín-Loeches, M. \& Molina, V. Gamma power and cognition in patients with schizophrenia and their first-degree relatives. Neuropsychobiology 69, 120-128 (2014).

283. Adachi, Y. et al. Functional connectivity between anatomically unconnected areas is shaped by collective network-level effects in the macaque cortex. Cereb. Cortex 22, 1586-1592 (2012).

284. Honey, C. J., Thivierge, J. P. \& Sporns, O. Can structure predict function in the human brain? Neurolmage 52, 766-776 (2010).

285. Knösche, T. R., Anwander, A., Liptrot, M. \& Dyrby, T. B. Validation of tractography: Comparison with manganese tracing. Hum. Brain Mapp. 36, 4116-4134 (2015).

286. Hein, K. H. M., Neher, P., Christophe, J. \& Alexandre, M. Tractography - based connectomes are dominated by false - positive connections. bioRxiv 1-23 (2016).

287. Thomas, C. et al. Anatomical accuracy of brain connections derived from diffusion MRI tractography is inherently limited. Proc. Natl. Acad. Sci. 111, 16574-16579 (2014).

288. Zalesky, A. et al. Connectome sensitivity or specificity: which is more important? Neuroimage 142, 407-420 (2016). 
289. Patel, S. et al. A meta-analysis of diffusion tensor imaging studies of the corpus callosum in schizophrenia. Schizophr. Res. 129, 149-155 (2011).

290. Alba-Ferrara, L. M. \& de Erausquin, G. A. What does anisotropy measure? Insights from increased and decreased anisotropy in selective fiber tracts in schizophrenia. Front. Integr. Neurosci. 7, (2013).

291. Kochunov, P. et al. Relationship between white matter fractional anisotropy and other indices of cerebral health in normal aging: Tract-based spatial statistics study of aging. Neuroimage 35, 478-487 (2007).

292. Jones, J. T. et al. Childhood-onset lupus with clinical neurocognitive dysfunction shows lower streamline density and pairwise connectivity on diffusion tensor imaging. Lupus 24, 1081-1086 (2015).

293. Baiano, M. et al. Anterior cingulate volumes in schizophrenia: A systematic review and a meta-analysis of MRI studies. Schizophr. Res. 93, 1-12 (2007).

294. Harrison, P. J. The hippocampus in schizophrenia: A review of the neuropathological evidence and its pathophysiological implications. Psychopharmacology 174, 151-162 (2004).

295. Pergola, G., Selvaggi, P., Trizio, S., Bertolino, A. \& Blasi, G. The role of the thalamus in schizophrenia from a neuroimaging perspective. Neuroscience and Biobehavioral Reviews 54, 57-75 (2015).

296. Simpson, E. H., Kellendonk, C. \& Kandel, E. A Possible Role for the Striatum in the Pathogenesis of the Cognitive Symptoms of Schizophrenia. Neuron 65, 585-596 (2010).

297. Barch, D. M. \& Ceaser, A. Cognition in schizophrenia: Core psychological and neural mechanisms. Trends in Cognitive Sciences 16, 27-34 (2012).

298. Sui, J. et al. In Search of Multimodal Neuroimaging Biomarkers of Cognitive Deficits in Schizophrenia. Biol. Psychiatry 78, 794-804 (2015).

299. Fuster, J. M. Synopsis of function and dysfunction of the frontal lobe. Acta Psychiatr. Scand. Suppl. 395, 51-7 (1999).

300. Fornito, A., Yücel, M., Patti, J., Wood, S. J. \& Pantelis, C. Mapping grey matter reductions in schizophrenia: An anatomical likelihood estimation analysis of voxel-based morphometry studies. Schizophr. Res. 108, 104-113 (2009).

301. Callicott, J. H. et al. Physiological dysfunction of the dorsolateral prefrontal cortex in schizophrenia revisited [In Process Citation]. Cereb Cortex 10, 1078-1092 (2000).

302. Fischer, B. A. et al. Cortical structural abnormalities in deficit versus nondeficit schizophrenia. Schizophr. Res. 136, 51-54 (2012).

303. Fuster, J. M. The prefrontal cortex. The Prefrontal Cortex (1997).

304. Buchsbaum, M. S. et al. Diffusion tensor imaging of frontal lobe white matter tracts in schizophrenia. Ann. Gen. Psychiatry 5 Article, (2006).

305. Mamah, D. et al. Anterior thalamic radiation integrity in schizophrenia: A diffusion-tensor imaging study. Psychiatry Res. - Neuroimaging 183, 144-150 (2010).

306. Rosenberger, G. et al. Anterior limb of the internal capsule in schizophrenia: A diffusion tensor tractography study. Brain Imaging Behav. 6, 417-425 (2012).

307. Wagner, G. et al. Structural and functional dysconnectivity of the fronto-thalamic system in schizophrenia: A DCM-DTI study. Cortex 66, 35-45 (2015). 
308. Kyriakopoulos, M. et al. Effect of age at onset of schizophrenia on white matter abnormalities. Br. J. Psychiatry 195, 346-353 (2009).

309. Hubl, D. et al. Pathways that make voices: White matter changes in auditory hallucinations. Arch. Gen. Psychiatry 61, 658-668 (2004).

310. Rotarska-Jagiela, A. et al. Anatomical brain connectivity and positive symptoms of schizophrenia: A diffusion tensor imaging study. Psychiatry Res. - Neuroimaging 174, 916 (2009).

311. Liu, X. et al. Reduced white matter integrity and cognitive deficit in never-medicated chronic schizophrenia: A diffusion tensor study using TBSS. Behav. Brain Res. 252, 157163 (2013).

312. James, A. et al. Abnormal frontostriatal connectivity in adolescent-onset schizophrenia and its relationship to cognitive functioning. Eur. Psychiatry 35, 32-38 (2016).

313. Kubicki, M. et al. Cingulate fasciculus integrity disruption in schizophrenia: A magnetic resonance diffusion tensor imaging study. Biol. Psychiatry 54, 1171-1180 (2003).

314. Cheung, V. et al. Positive symptoms and white matter microstructure in never-medicated first episode schizophrenia. Psychol. Med. 41, 1709-1719 (2011).

315. Filippi, M. et al. Patterns of brain structural changes in first-contact, antipsychotic drugnaive patients with schizophrenia. AJNR. Am. J. Neuroradiol. 35, 30-7 (2014).

316. Szeszko, P. R. et al. Clinical and neuropsychological correlates of white matter abnormalities in recent onset schizophrenia. Neuropsychopharmacology 33, 976-84 (2008).

317. Caprihan, A. et al. The Paradoxical Relationship between White Matter, Psychopathology and Cognition in Schizophrenia: A Diffusion Tensor and Proton Spectroscopic Imaging Study. Neuropsychopharmacology 40, 2248-2257 (2015).

318. Kitamura, H. et al. Diffusion tensor analysis in chronic schizophrenia a preliminary study on a high-field (3.0T) system. Eur. Arch. Psychiatry Clin. Neurosci. 255, 313-318 (2005).

319. Kumra, S. et al. White matter abnormalities in early-onset schizophrenia: A voxel-based diffusion tensor imaging study. J. Am. Acad. Child Adolesc. Psychiatry 44, 934-941 (2005).

320. Minami, T. et al. Diffusion tensor magnetic resonance imaging of disruption of regional white matter in schizophrenia. Neuropsychobiology 47, 141-5 (2003).

321. Voineskos, A. N. et al. Neuroimaging evidence for the deficit subtype of schizophrenia. JAMA Psychiatry 70, 472-480 (2013).

322. Friedman, J. I. et al. Diffusion tensor imaging findings in first-episode and chronic schizophrenia patients. Am. J. Psychiatry 165, 1024-32 (2008).

323. Andreasen, N. C. et al. Progressive brain change in schizophrenia: A prospective longitudinal study of first-episode schizophrenia. Biol. Psychiatry 70, 672-679 (2011).

324. Canu, E., Agosta, F. \& Filippi, M. A selective review of structural connectivity abnormalities of schizophrenic patients at different stages of the disease. Schizophrenia Research 161, 19-28 (2015).

325. Knöchel, C. et al. Association between white matter fiber integrity and subclinical psychotic symptoms in schizophrenia patients and unaffected relatives. Schizophr. Res. 140, 129-135 (2012).

326. Bartzokis, G. et al. Differential effects of typical and atypical antipsychotics on brain myelination in schizophrenia. Schizophr. Res. 93, 13-22 (2007). 
327. Tognoli, E. \& Kelso, J. A. S. The metastable brain. Neuron 81, 35-48 (2014).

328. Tan, H. R. M., Lana, L. \& Uhlhaas, P. J. High-frequency neural oscillations and visual processing deficits in schizophrenia. Front. Psychol. 4, 1-19 (2013).

329. Micheloyannis, S. World Journal of Psychiatry. 2, (2012).

330. Smit, D. J. A., Stam, C. J., Posthuma, D., Boomsma, D. I. \& de Geus, E. J. C. Heritability of 'small-world' networks in the brain: a graph theoretical analysis of resting-state EEG functional connectivity. Hum. Brain Mapp. 29, 1368-78 (2008).

331. Li, M., Chen, Z. \& Li, T. Small-world brain networks in schizophrenia. Shanghai Arch. psychiatry 24, 322-7 (2012).

332. Buzsáki, G. Diversity of Cortical Functions. in Rythms of the Brain 21-79 (Oxford University Press: New York, 2006).

333. Wang, Q. et al. Anatomical insights into disrupted small-world networks in schizophrenia. Neuroimage 59, 1085-93 (2012).

334. Bassett, D. S. et al. Hierarchical organization of human cortical networks in health and schizophrenia. J. Neurosci. 28, 9239-48 (2008).

335. He, H. et al. Altered small-world brain networks in schizophrenia patients during working memory performance. PLoS One 7, e38195 (2012).

336. Fazzari, P. et al. Control of cortical GABA circuitry development by Nrg1 and ErbB4 signalling. Nature 464, 1376-80 (2010).

337. Calaora, V. et al. Neuregulin signaling regulates neural precursor growth and the generation of oligodendrocytes in vitro. J. Neurosci. 21, 4740-4751 (2001).

338. Flames, N. et al. Short- and long-range attraction of cortical GABAergic interneurons by neuregulin-1. Neuron 44, 251-61 (2004).

339. Harrison, P. J. \& Law, A. J. Neuregulin 1 and Schizophrenia: Genetics, Gene Expression, and Neurobiology. Biol. Psychiatry 60, 132-140 (2006).

340. Kwon, O. Bin et al. Neuregulin-1 regulates LTP at CA1 hippocampal synapses through activation of dopamine D4 receptors. Proc. Natl. Acad. Sci. U. S. A. 105, 15587-15592 (2008).

341. Banerjee, A., MacDonald, M. L., Borgmann-Winter, K. E. \& Hahn, C. G. Neuregulin 1-erbB4 pathway in schizophrenia: From genes to an interactome. Brain Res. Bull. 83, 132-139 (2010).

342. Stefansson, H. et al. Neuregulin 1 and susceptibility to schizophrenia. Am. J. Hum. Genet. 71, 877-892 (2002).

343. Keri, S., Kiss, I., Seres, I. \& Kelemen, O. A polymorphism of the neuregulin 1 gene(SNP8NRG243177/rs6994992) affects reactivity to expressed emotion in schizophrenia. Am. J. Med. Genet. Part B Neuropsychiatr. Genet. 150, 418-420 (2009).

344. McBain, C. J. \& Fisahn, A. Interneurons unbound. Nat. Rev. Neurosci. 2, 11-23 (2001).

345. Mclntosh, a M. et al. The effects of a neuregulin 1 variant on white matter density and integrity. Mol. Psychiatry 13, 1054-1059 (2008).

346. Buonanno, A. The neuregulin signaling pathway and schizophrenia: From genes to synapses and neural circuits. Brain Res. Bull. 83, 122-131 (2010).

347. Cho, Y. et al. Effects of genetic variations in NRG1 on cognitive domains in patients with schizophrenia and healthy individuals. Psychiatr. Genet. (2015). 
348. Hall, J. et al. A neuregulin 1 variant associated with abnormal cortical function and psychotic symptoms. Nat. Neurosci. 9, 1477-1478 (2006).

349. Goes, F. S. et al. Family-based association study of Neuregulin 1 with psychotic bipolar disorder. Am. J. Med. Genet. B. Neuropsychiatr. Genet. 150B, 693-702 (2009).

350. Bledowski, C. et al. Localizing P300 Generators in Visual Target and Distractor Processing: A Combined Event-Related Potential and Functional Magnetic Resonance Imaging Study. J. Neurosci. 24, 9353-60 (2004).

351. Bachiller, A. et al. A comparative study of event-related coupling patterns during an auditory oddball task in schizophrenia. J. Neural Eng. 12, 16007 (2015).

352. Stéphane Mallat. In A Wavelet Tour of Signal Processing. in A Wavelet Tour of Signal Processing 20-41 (1999).

353. Torrence, C. \& Compo, G. P. A practical guide to wavelet analysis. Bams 79, 61 (1998).

354. Nunez, P. L. et al. EEG coherency. I: Statistics, reference electrode, volume conduction, Laplacians, cortical imaging, and interpretation at multiple scales. Electroencephalogr. Clin. Neurophysiol. 103, 499-515 (1997).

355. Boccaletti, S., Latora, V., Moreno, Y., Chavez, M. \& Hwang, D. U. Complex networks: Structure and dynamics. Phys. Rep. 424, 175-308 (2006).

356. Stam, C. J. et al. Graph theoretical analysis of magnetoencephalographic functional connectivity in Alzheimer's disease. Brain 132, 213-24 (2009).

357. Rubinov, M. \& Sporns, O. Complex network measures of brain connectivity: uses and interpretations. Neuroimage 52, 1059-69 (2010).

358. Bachiller, A. et al. Decreased entropy modulation of EEG response to novelty and relevance in schizophrenia during a P300 task. Eur. Arch. Psychiatry Clin. Neurosci. (2014).

359. von Stein, A. \& Sarnthein, J. Different frequencies for different scales of cortical integration: from local gamma to long range alpha/theta synchronization. Int. J. Psychophysiol. 38, 301-13 (2000).

360. Siegel, M., Donner, T. H. \& Engel, A. K. Spectral fingerprints of large-scale neuronal interactions. Nat. Rev. Neurosci. 13, 121-34 (2012).

361. Mothersill, O., Kelly, S., Rose, E. J. \& Donohoe, G. The effects of psychosis risk variants on brain connectivity: a review. Front. psychiatry 3, 18 (2012).

362. Sprooten, E. et al. The relationship of anterior thalamic radiation integrity to psychosis risk associated neuregulin-1 variants. Mol. Psychiatry 14, 237-8, 233 (2009).

363. Uhlhaas, P. J. \& Singer, W. Neuronal dynamics and neuropsychiatric disorders: toward a translational paradigm for dysfunctional large-scale networks. Neuron 75, 963-80 (2012).

364. Uhlhaas, P. J., Haenschel, C., Nikolić, D. \& Singer, W. The role of oscillations and synchrony in cortical networks and their putative relevance for the pathophysiology of schizophrenia. Schizophr. Bull. 34, 927-943 (2008).

365. Law, A. J. et al. Neuregulin 1 transcripts are differentially expressed in schizophrenia and regulated by 5 ' SNPs associated with the disease. Proc. Natl. Acad. Sci. U. S. A. 103, 674752 (2006).

366. Hahn, C.-G. et al. Altered neuregulin 1-erbB4 signaling contributes to NMDA receptor hypofunction in schizophrenia. Nat. Med. 12, 824-8 (2006).

367. Gu, Z., Jiang, Q., Fu, A. K. Y., Ip, N. Y. \& Yan, Z. Regulation of NMDA receptors by 
neuregulin signaling in prefrontal cortex. J. Neurosci. 25, 4974-84 (2005).

368. Bjarnadottir, M. et al. Neuregulin1 (NRG1) signaling through Fyn modulates NMDA receptor phosphorylation: differential synaptic function in NRG1+/- knock-outs compared with wild-type mice. J. Neurosci. 27, 4519-29 (2007).

369. Woo, R.-S. S. et al. Neuregulin-1 Enhances Depolarization-Induced GABA Release. Neuron 54, 599-610 (2007).

370. Ting, A. K. et al. Neuregulin 1 promotes excitatory synapse development and function in GABAergic interneurons. J. Neurosci. 31, 15-25 (2011).

371. Lewis, D. A. \& Moghaddam, B. Cognitive dysfunction in schizophrenia: convergence of gamma-aminobutyric acid and glutamate alterations. Arch. Neurol. 63, 1372-6 (2006).

372. Bramon, E. et al. Neuregulin-1 and the P300 waveform-A preliminary association study using a psychosis endophenotype. Schizophr. Res. 103, 178-185 (2008).

373. Uhlhaas, P. J. Neural dynamics in mental disorders. World Psychiatry 14, 116-8 (2015).

374. Braff, D. L., Freedman, R., Schork, N. J. \& Gottesman, I. I. Deconstructing schizophrenia: An overview of the use of endophenotypes in order to understand a complex disorder. Schizophr. Bull. 33, 21-32 (2007).

375. Owens, E. M., Bachman, P., Glahn, D. C. \& Bearden, C. E. Electrophysiological Endophenotypes for Schizophrenia. Harvard Review of Psychiatry 24, 129-147 (2016).

376. Turetsky, B. I. et al. The utility of P300 as a schizophrenia endophenotype and predictive biomarker: Clinical and socio-demographic modulators in COGS-2. Schizophr. Res. 163, 53-62 (2015).

377. Micheloyannis, S. Graph-based network analysis in schizophrenia. World J. Psychiatry 2, 1 (2012).

378. Díez, A. et al. Cognitive outcome and gamma noise power unrelated to neuregulin 1 and 3 variation in schizophrenia. Ann. Gen. Psychiatry 13, 18 (2014).

379. Bassett, D. S. et al. Cognitive fitness of cost-efficient brain functional networks. Proc. Natl. Acad. Sci. U. S. A. 106, 11747-52 (2009).

380. Achard, S. \& Bullmore, E. Efficiency and cost of economical brain functional networks. PLoS Comput. Biol. 3, e17 (2007).

381. Walton, E., Turner, J. A. \& Ehrlich, S. Neuroimaging as a potential biomarker to optimize psychiatric research and treatment. Int. Rev. Psychiatry 25, 619-631 (2013).

382. Smit, D. J. A., Posthuma, D., Boomsma, D. I. \& De Geus, E. J. C. Heritability of background EEG across the power spectrum. Psychophysiology 42, 691-697 (2005).

383. Van Beijsterveldt, C. E. M. \& Van Baal, G. C. M. Twin and family studies of the human electroencephalogram: A review and a meta-analysis. Biological Psychology 61, 111-138 (2002).

384. De Geus, E. J. C. From genotype to EEG endophenotype: A route for post-genomic understanding of complex psychiatric disease? Genome Medicine 2, (2010).

385. Schutte, N. M. et al. Heritability of resting state EEG functional connectivity patterns. Twin Res. Hum. Genet. 16, 962-969 (2013).

386. Pardiñas, A. F. et al. Common schizophrenia alleles are enriched in mutation-intolerant genes and in regions under strong background selection. Nat. Genet. 50, 381-389 (2018).

387. Narayanan, B. et al. Multivariate genetic determinants of EEG oscillations in 
schizophrenia and psychotic bipolar disorder from the BSNIP study. Transl. Psychiatry 5, (2015).

388. Del Re, E. C. et al. Analysis of schizophrenia-related genes and electrophysiological measures reveals ZNF804A association with amplitude of P300b elicited by novel sounds. Transl. Psychiatry 4, (2014).

389. Kang, C. et al. Association study of neuregulin 1 gene polymorphisms with auditory $\mathrm{p} 300$ in schizophrenia. Am. J. Med. Genet. Part B Neuropsychiatr. Genet. 159 B, 422-428 (2012).

390. Striessnig, J., Pinggera, A., Kaur, G., Bock, G. \& Tuluc, P. L-type Ca2+ channels in heart and brain. Wiley Interdiscip. Rev. Membr. Transp. Signal. 3, 15-38 (2014).

391. Gomez-Ospina, N., Tsuruta, F., Barreto-Chang, O., Hu, L. \& Dolmetsch, R. The C Terminus of the L-Type Voltage-Gated Calcium Channel CaV1.2 Encodes a Transcription Factor. Cell 127, 591-606 (2006).

392. Simms, B.A., and Zamponi, W. Neuronal voltage-gated calcium channels: Structure, function, and dysfunction. Neuron 82, 24-45 (2014).

393. Saliba, R. S., Gu, Z., Yan, Z. \& Moss, S. J. Blocking L-type voltage-gated Ca2+ channels with dihydropyridines reduces ??-aminobutyric acid type A receptor expression and synaptic inhibition. J. Biol. Chem. 284, 32544-32550 (2009).

394. Kabir, Z. D. et al. Rescue of impaired sociability and anxiety-like behavior in adult cacna1cdeficient mice by pharmacologically targeting elF2 $\alpha$. Mol. Psychiatry 22, 1096-1109 (2017).

395. Cross-Disorder Group of the Psychiatric Genomics Consortium, C.-D. G. of the P. G. Identification of risk loci with shared effects on five major psychiatric disorders: a genome-wide analysis. Lancet (London, England) 381, 1371-9 (2013).

396. Ripke, S. et al. Biological insights from 108 schizophrenia-associated genetic loci. Nature 511, 421-427 (2014).

397. Guan, F. et al. MIR137 gene and target gene CACNA1C of miR-137 contribute to schizophrenia susceptibility in Han Chinese. Schizophr. Res. 152, 97-104 (2014).

398. He, K. et al. CACNA1C, schizophrenia and major depressive disorder in the Han Chinese population. Br. J. Psychiatry 204, 36-9 (2014).

399. Ivorra, J. L. et al. Replication of previous genome-wide association studies of psychiatric diseases in a large schizophrenia case-control sample from Spain. Schizophr. Res. 159, 107-113 (2014).

400. Nie, F. et al. Genetic analysis of SNPs in CACNA1C and ANK3 gene with schizophrenia: A comprehensive meta-analysis. Am. J. Med. Genet. Part B Neuropsychiatr. Genet. 168, 637-648 (2015).

401. Bigos, K. L. et al. Genetic variation in CACNA1C affects brain circuitries related to mental illness. Arch. Gen. Psychiatry 67, 939-945 (2010).

402. Green, E. K. et al. The bipolar disorder risk allele at CACNA1C also confers risk of recurrent major depression and of schizophrenia. Mol Psychiatry 15, 1016-1022 (2010).

403. Nyegaard, M. et al. CACNA1C (rs1006737) is associated with schizophrenia. Molecular Psychiatry 15, 119-121 (2010).

404. Wolf, C. et al. CACNA1C genotype explains interindividual differences in amygdala volume among patients with schizophrenia. Eur. Arch. Psychiatry Clin. Neurosci. 264, 93- 
102 (2014).

405. Wang, F., Mcintosh, A. M., He, Y., Gelernter, J. \& Blumberg, H. P. The association of genetic variation in CACNA1C with structure and function of a frontotemporal system. Bipolar Disord. 13, 696-700 (2011).

406. Lancaster, T. M., Foley, S., Tansey, K. E., Linden, D. E. J. \& Caseras, X. CACNA1C risk variant is associated with increased amygdala volume. Eur. Arch. Psychiatry Clin. Neurosci. 266, 269-275 (2016).

407. Huang, L. et al. The impact of CACNA1C allelic variation on regional gray matter volume in Chinese population. Am. J. Med. Genet. B. Neuropsychiatr. Genet. 171B, 396-401 (2016).

408. Erk, S. et al. Brain function in carriers of a genome-wide supported bipolar disorder variant. Arch. Gen. Psychiatry 67, 803-811 (2010).

409. Paulus, F. M. et al. Association of rs1006737 in CACNA1C with alterations in prefrontal activation and fronto-hippocampal connectivity. Hum. Brain Mapp. 35, 1190-1200 (2014).

410. Gurung, R. \& Prata, D. P. What is the impact of genome-wide supported risk variants for schizophrenia and bipolar disorder on brain structure and function? A systematic review. Psychological Medicine 45, 2461-2480 (2015).

411. Radua, J. et al. The impact of CACNA1C allelic variation on effective connectivity during emotional processing in bipolar disorder. Molecular Psychiatry 18, 526-527 (2013).

412. Eckart, N. et al. Functional characterization of schizophrenia-associated variation in CACNA1C. PLoS One 11, (2016).

413. Yoshimizu, T. et al. Functional implications of a psychiatric risk variant within CACNA1C in induced human neurons. Mol. Psychiatry 20, 162-169 (2015).

414. Ji, H. et al. Functional characterization of ether-a-go-go-related gene potassium channels in midbrain dopamine neurons - implications for a role in depolarization block. Eur. J. Neurosci. 36, 2906-2916 (2012).

415. Pessia, M. et al. ERG voltage-gated K+ channels regulate excitability and discharge dynamics of the medial vestibular nucleus neurones. J. Physiol. 586, 4877-4890 (2008).

416. Huffaker, S. J. et al. A primate-specific, brain isoform of $\mathrm{KCNH} 2$ affects cortical physiology, cognition, neuronal repolarization and risk of schizophrenia. Nat. Med. 15, 509-518 (2009).

417. Hashimoto, R. et al. The $\mathrm{KCNH} 2$ gene is associated with neurocognition and the risk of schizophrenia. World J. Biol. Psychiatry 14, 114-120 (2013).

418. Carr, G. V. et al. KCNH2-3.1 expression impairs cognition and alters neuronal function in a model of molecular pathology associated with schizophrenia. Mol. Psychiatry 21, (2016).

419. Gomez-Pilar, J. et al. Neural Network Reorganization Analysis During an Auditory Oddball Task in Schizophrenia Using Wavelet Entropy. Entropy 17, 5241-5256 (2015).

420. Tallon-Baudry, C., Bertrand, O., Delpuech, C. \& Pernier, J. Stimulus specificity of phaselocked and non-phase-locked $40 \mathrm{~Hz}$ visual responses in human. J. Neurosci. 16, 42404249 (1996).

421. Cosgrove, D. et al. Cognitive characterization of schizophrenia risk variants involved in synaptic transmission: Evidence of CACNA1C's role in working memory. 
Neuropsychopharmacology 42, 2612-2622 (2017).

422. Zhang, S. Y. et al. Role of CACNA1C gene polymorphisms and protein expressions in the pathogenesis of schizophrenia: a case-control study in a Chinese population. Neurol. Sci. 38, 1393-1403 (2017).

423. Woon, P. S. et al. CACNA1C genomewide supported psychosis genetic variation affects cortical brain white matter integrity in chinese patients with schizophrenia. J. Clin. Psychiatry 75, e1284-e1290 (2014).

424. Dedic, N. et al. Cross-disorder risk gene CACNA1C differentially modulates susceptibility to psychiatric disorders during development and adulthood. Mol. Psychiatry 23, 533-543 (2018).

425. Krautheim, J. T. et al. Outgroup emotion processing in the VACC is modulated by childhood trauma and CACNA1C risk variant. Soc. Cogn. Affect. Neurosci. 13, 341-348 (2018).

426. Ward, L. D. \& Kellis, M. HaploReg: A resource for exploring chromatin states, conservation, and regulatory motif alterations within sets of genetically linked variants. Nucleic Acids Res. 40, (2012).

427. Lubeiro, A. et al. Identification of two clusters within schizophrenia with different structural, functional and clinical characteristics. Prog. Neuro-Psychopharmacology Biol. Psychiatry 64, (2016).

428. Honey, C. J. et al. Predicting human resting-state functional connectivity from structural connectivity. Proc. Natl. Acad. Sci. U. S. A. 106, 2035-40 (2009).

429. R. Dauvermann, M. et al. Relationship Between Gyrification and Functional Connectivity of the Prefrontal Cortex in Subjects at High Genetic Risk of Schizophrenia. Curr. Pharm. Des. 18, 434-442 (2012).

430. Palaniyappan, L. \& Liddle, P. F. Aberrant cortical gyrification in schizophrenia: Palaniyappan, L., \& Liddle, P. F. (2012). Aberrant cortical gyrification in schizophrenia: A surface-based morphometry study. Journal of Psychiatry and Neuroscience, 37(6), 399406. J. Psychiatry Neurosci. 37, 399-406 (2012).

431. Palaniyappan, L. et al. Gyrification of Broca's region is anomalously lateralized at onset of schizophrenia in adolescence and regresses at 2year follow-up. Schizophr. Res. 147, 39-45 (2013).

432. Tepest, R. et al. Morphometry of structural disconnectivity indicators in subjects at risk and in age-matched patients with schizophrenia. Eur. Arch. Psychiatry Clin. Neurosci. 263, 15-24 (2013).

433. Sasabayashi, D. et al. Increased frontal gyrification negatively correlates with executive function in patients with first-episode schizophrenia. Cereb. Cortex 27, 2686-2694 (2017).

434. Neilson, E. et al. Polygenic risk for schizophrenia, transition and cortical gyrification: a high-risk study. Psychological Medicine 1-11 (2017).

435. Liu, B. et al. Polygenic risk for schizophrenia influences cortical gyrification in 2 independent general populations. Schizophr. Bull. 43, 673-680 (2017).

436. Mclntosh, A. M. et al. Prefrontal gyral folding and its cognitive correlates in bipolar disorder and schizophrenia. Acta Psychiatr. Scand. 119, 192-198 (2009).

437. Mancini-Marïe, A. et al. Sex, age, symptoms and illness duration and their relation with 
gyrification index in schizophrenia. Clin. Schizophr. Relat. Psychoses 150728091824005 (2015).

438. Cao, B. et al. Lifespan Gyrification Trajectories of Human Brain in Healthy Individuals and Patients with Major Psychiatric Disorders. Sci. Rep. 7, 511 (2017).

439. Palaniyappan, L. et al. Cortical folding defects as markers of poor treatment response in first-episode psychosis. JAMA Psychiatry 70, 1031-1040 (2013).

440. Zuliani, R. et al. Increased gyrification in schizophrenia and non affective first episode of psychosis. Schizophr. Res. 193, 269-275 (2018).

441. Honnorat, N., Dong, A., Meisenzahl-Lechner, E., Koutsouleris, N. \& Davatzikos, C. Neuroanatomical heterogeneity of schizophrenia revealed by semi-supervised machine learning methods. Schizophrenia Research (2017).

442. Hirjak, D. et al. Cortical features of distinct developmental trajectories in patients with delusional infestation. Prog. Neuro-Psychopharmacology Biol. Psychiatry 76, 72-79 (2017).

443. Weinberg, D. et al. Cognitive subtypes of schizophrenia characterized by differential brain volumetric reductions and cognitive decline. JAMA Psychiatry 73, 1251-1259 (2016).

444. Wexler, B. E. et al. Neuropsychological near normality and brain structure abnormality in schizophrenia. Am. J. Psychiatry 166, 189-195 (2009).

445. MacCabe, J. H., Aldouri, E., Fahy, T. A., Sham, P. C. \& Murray, R. M. Do schizophrenic patients who managed to get to university have a non-developmental form of illness? Psychol. Med. 32, 535-544 (2002).

446. Mistry, S., Harrison, J. R., Smith, D. J., Escott-Price, V. \& Zammit, S. The use of polygenic risk scores to identify phenotypes associated with genetic risk of schizophrenia: Systematic review. Schizophrenia Research (2017).

447. Kumar, D. et al. Cacna1c (Cav1.2) Modulates Electroencephalographic Rhythm and Rapid Eye Movement Sleep Recovery. Sleep 38, 1371-1380 (2015).

448. Quidé, Y., Morris, R. W., Shepherd, A. M., Rowland, J. E. \& Green, M. J. Task-related fronto-striatal functional connectivity during working memory performance in schizophrenia. Schizophr. Res. 150, 468-475 (2013).

449. Wu, S. et al. Task performance modulates functional connectivity involving the dorsolateral prefrontal cortex in patients with schizophrenia. Front. Psychol. 8, (2017)

450. Clementz, B. A. et al. Identification of distinct psychosis biotypes using brain-based biomarkers. Am. J. Psychiatry 173, 373-384 (2016).

451. Meda, S. A. et al. Examining Functional Resting-State Connectivity in Psychosis and Its Subgroups in the Bipolar-Schizophrenia Network on Intermediate Phenotypes Cohort. Biol. Psychiatry Cogn. Neurosci. Neuroimaging 1, 488-497 (2016). 